\title{
A THEORY OF
}

\section{LITERATE ACTION:}

PRONADED B

Wh

\section{VOLUME 2}

MORE READHX

EXN CT CERTA

A

Ittis $N$ Not

b) trate

a)

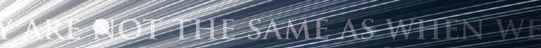

-TWA TAT PRESENT-DAY WRTIER

AFED IN DEJAIL WHEN WE COME TO

PEECH ITSELF WHEN WE HAVE PROVED

MABLE TO THE CASE IN QUESTION N. THEF

IS TO BE IN COMMAND OF THEMAM

AP
$C A$
$P O$

Wharacter aND GoOdN

NAM NABM AND DESCRIBE THEM,

APREA $2 S$ MATRHETORIC IS AN OFFSHOO-

CA

ot LE MAMAMLNGS. AS A MATtER O

out

FA

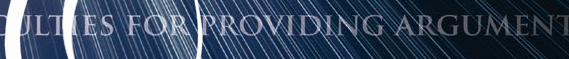

TEL TO EA AM OWMER. WI TII REGARI

AS

Perspectives

on Writing

Charles Bazerman

IS AN APPARENT SHMOCOSM.M CALL THE

INDUCTION. EVERY QN EE MAO DEFEAC 



\section{A THEORY OF LITERATE ACTION: LITERATE ACTION VOLUME 2}




\section{PERSPECTIVES ON WRITING}

Series Editor, Susan H. McLeod

The Perspectives on Writing series addresses writing studies in a broad sense. Consistent with the wide ranging approaches characteristic of teaching and scholarship in writing across the curriculum, the series presents works that take divergent perspectives on working as a writer, teaching writing, administering writing programs, and studying writing in its various forms.

The WAC Clearinghouse and Parlor Press are collaborating so that these books will be widely available through free digital distribution and low-cost print editions. The publishers and the Series editor are teachers and researchers of writing, committed to the principle that knowledge should freely circulate. We see the opportunities that new technologies have for further democratizing knowledge. And we see that to share the power of writing is to share the means for all to articulate their needs, interest, and learning into the great experiment of literacy.

\section{Recent Books in the Series}

Katherine V. Wills and Rich Rice (Eds.), ePortfolio Performance Support Systems: Constructing, Presenting, and Assessing Portfolios (2013)

Mike Duncan and Star Medzerian Vanguri (Eds.), The Centrality of Style (2013)

Chris Thaiss, Gerd Bräuer, Paula Carlino, Lisa Ganobcsik-Williams, and Aparna Sinha (Eds.), Writing Programs Worldwide: Profiles of Academic Writing in Many Places (2012)

Andy Kirkpatrick and Zhichang Xu, Chinese Rhetoric and Writing: An Introduction for Language Teachers (2012)

Doreen Starke-Meyerring, Anthony Paré, Natasha Artemeva, Miriam Horne, and Larissa Yousoubova (Eds.), Writing in Knowledge Societies (2011)

Martine Courant Rife, Shaun Slattery, and Dànielle Nicole DeVoss (Eds.), Copy (write): Intellectual Property in the Writing Classroom (2011)

David Franke, Alex Reid, and Anthony Di Renzo (Eds.), Design Discourse: Composing and Revising Programs in Professional and Technical Writing (2010) 


\section{A THEORY OF LITERATE ACTION: LITERATE ACTION VOLUME 2}

By Charles Bazerman

The WAC Clearinghouse

wac.colostate.edu

Fort Collins, Colorado

Parlor Press

www.parlorpress.com

Anderson, South Carolina 
The WAC Clearinghouse, Fort Collins, Colorado 80523

Parlor Press, 3015 Brackenberry Drive, Anderson, South Carolina 29621

(C) 2013 by Charles Bazerman. This work is licensed under a Creative Commons Attribution-Noncommercial-No Derivative Works 3.0 United States License.

ISBN 978-1-64215-053-7 (pdf) | 978-1-64215-053474 (epub) | 978-1-60235-477-7 (pbk.)

DOI 10.37514/PER-B.2013.4791

Produced in the United States of America

Library of Congress Cataloging-in-Publication Data

A Theory of Literate Action: Literate Action Volume 2 / by Charles Bazerman. vii, 217 pages ; $24 \mathrm{~cm}$. -- (Perspectives on writing)

Includes bibliographical references.

ISBN 978-1-60235-477-7 (pbk.: acid-free paper) -- ISBN 978-1-60235-478-4 (hardcover: acid-free paper) -- ISBN 978-1-64215-053-7 (pdf) -- ISBN 978-1-64215-053474 (epub)

1. Rhetoric. 2. Written communication. I. Title.

P301.B366 2013

2013047451

Copyeditor: Don Donahue

Designer: Mike Palmquist

Series Editor: Susan H. McLeod

The WAC Clearinghouse supports teachers of writing across the disciplines. Hosted by Colorado State University, it brings together scholarly journals and book series as well as resources for teachers who use writing in their courses. This book is available in digital format for free download at wac.colostate.edu.

Parlor Press, LLC is an independent publisher of scholarly and trade titles in print and multimedia formats. This book is available in paperback, cloth, and Adobe eBook formats from Parlor Press at www.parlorpress.com. For submission information or to find out about Parlor Press publications, write to Parlor Press, 3015 Brackenberry Drive, Anderson, South Carolina 29621, or e-mail editor@parlorpress.com. 


\section{CONTENTS}

Introduction...$\ldots \ldots \ldots \ldots \ldots \ldots \ldots \ldots \ldots \ldots \ldots \ldots \ldots$

Chapter 1. The Symbolic Animal and the Cultural Transformation of

Nature..................................

Writing as Learned Activity. . . . . . . . . . . . . . . . 7

Activity, Work, and Transformation of Consciousness . . . . . . . .9

Biological and Cultural Evolution...................13

The Transformed and Extended Here and Now. . . . . . . . . . . 14

Non-Symbolic and Symbolic Cognition ................. . 16

Language as Situated, Embodied Utterance. . . . . . . . . . . . . . 19

Language, Literacy, and Cognitive Development.............21

Chapter 2. Symbolic Selves in Society: Vygotsky on Language and

Formation of the Social Mind .......................... 5

Linguistic Structure and Literary Affect: Vygotsky's Catharsis . . . . .26

Goals, Obstacles, and Empowerment: Vygotsky's Adler ........28

Cognitive Tools.............................. 30

Second Order Symbol Systems and Consciousness Development . .34 Interaction and Self-Regulation: Influencing Others and Influencing

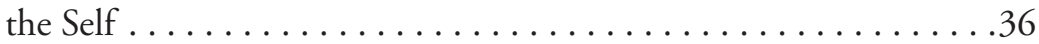

Places of Play, Self-Articulation, and Development . . . . . . . . 39

Chapter 3. Active Social Symbolic Selves: Vygotskian Traditions. . . . . .43

Activity, Object, Affect, and Social System: Leont'ev . . . . . . . . . . . 44

Complex Activity Systems: Engeström . . . . . . . . . . . . . . .47

Written Genres in Activity Systems . . . . . . . . . . . . . 52

Reflectivity in Individual and Group Writing Activity. . . . . . . . 55

Meaning, Consciousness, and Activity: Luria . . . . . . . . . . 56

Chapter 4. Active Social Symbolic Selves: The Phenomenological

Sociology Tradition ........................65

Schutz, the Problems of Economic Behavior, and a Unified Social

Science ...............................66

Schutz's Typification . . . . . . . . . . . . . . . .68

The Typified Internal and External, and the Falling Away of the

Untypifiable .......................... . .

Relevance in Consciousness and Externalized Mediations . . . . . . .72

The Natural Attitude and the Pull of Typified Consciousness . . . . 73

Critiques of Social Construction and Ways Out ............76 
Typification, Novelty, and Particularized Meanings . . . . . . . . . 77

Typification and Particularity: Appreciating the Music of Life . . . . 79

Schutz, Berger, and Luckman and the Social Production of the

Everyday........................... 82

Chapter 5. Active Social Symbolic Selves: The Pragmatic Tradition within

American Social Science. . . . . . . . . . . . . . . . . . . . 8 85

Philosophic Pragmatism . . . . . . . . . . . . . . . . . 86

Pragmatism as a Perspective for Social Understanding

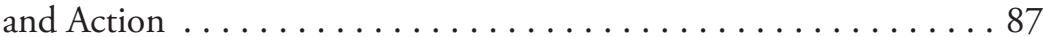

The Differences and Commonalities of Early Pragmatisms . . . . . 88

Peirce's Semiotics with Interpretation . . . . . . . . . . . . . 89

James's Psychology of Experience. . . . . . . . . . . . . . . . . . 90

Dewey's Thinking about Problems . . . . . . . . . . . . 90

Dewey's Learning through Active Experience . . . . . . . . . . . . 91

The Problem of Living with Others: Mead . . . . . . . . . 93

Mead in Relation to Other Traditions . . . . . ........... 94

Mead, Chicago Sociology, and Symbolic Interactionism . . . . . . . 97

Legal Institutions and Legal Practice as Experiment: Holmes . . . . 98

Pragmatic Influences on Sapir and Linguistic Anthropology . . . . 100

Sullivan's Pragmatic Interpersonal Psychiatry . . . . . . . . . 102

Language and Writing as Interpersonal and Self-Forming . . . . . . 104

Chapter 6. Social Order: Structural and Structurational Sociology . . . . 107

Merton's Social Structure through Individual Choice-Making. . . 107

The Mechanisms of Choice Making within Opportunity

Structures . . . . . . . . . . . . . . . . . . . . . . . . . . 109

Terms for an Agentive Structural Sociology . . . . . . . . . . 110

Merton's Relation to Structurationist Accounts . . . . . . . . . . . . 112

The Relevance of Meso-Phenomena and Theories of the Middle Range for Rhetoric and Writing. . . . . . . . . . . . . . . 115

Chapter 7. From the Interaction Order to Shared Meanings ....... 121

The Interaction Order . . . . . . . . . . . . . . . . . . . . . . 122

Proximate Interactional Orders and Distant . . . . . . . . . . . 124

Fragility of Written Interaction . . . . . . . . . . . . . 125

The Invisibility of Fragility . . . . . . . . . . . . . . . . . 127

Creating Alignment and Readability in Writing .......... 130

The Interactional Potential and Challenges of Evoking Novel

Meanings ............................ 131 


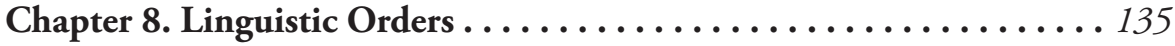

The Importance of the Orderliness of Language . . . . . . . . . 136

Creating Orderliness of Language . . . . . . . . . . . . . 138

Keeping Language Orderly: Housekeeping and Prescription . . . . 140

Learning Transcription . . . . . . . . . . . . . . . . . 142

Words and Lexical Orders . . . . . . . . . . . . . . . . . . . . 144

Syntax and Grammar, Ordering Word Relations . . . . . . . . . . 146

The Educational Uses of Rules . . . . . . . . . . . . . . . . 148

Chapter 9. Utterances and Their Meanings............... 151

Volosinov and His Circle's Proposal for an Utterance-

Based Linguistics. . . . . . . . . . . . . . . . . . . . . . 151

Utterance to Speech Act . . . . . . . . . . . . . . . . . . . 153

Social Facts . . . . . . . . . . . . . . . . . . . 156

Meaning in the Situated Speech Act World . . . . . . . . . . 158

Meaning from an Utterance Perspective . . . . . . . . . . 160

Sense-Making in Everyday Life . . . . . . . . . . . . . . . 162

The Sense-Making Difficulties of Literate Interaction . . . . . . . . 164

Socialization into Literate Worlds . . . . . . . . . . . . . . 165

Chapter 10. The World in the Text: Indexed and Created......... 169

Locutionary Acts, Ideational Functions, Chronotopes . . . . . . 170

Genred Ontologies and the Work of Expanding the

Worldview of the Genre .......................... 171

Epistemology, Accountability, and Trust . . . . . . . . . 173

Scientific Epistemologies, Methods, and Visible Phenomena. . . . 174

Pointing at Other Texts: Intertextuality . . . . . . . . . 176

The Intertext as a Resource and a Contended Topic . . . . . . . . 177

Intertextuality and Socially-Formed Consciousness . . . . . . . . 178

Intertextuality and Individuation $\ldots \ldots \ldots \ldots \ldots \ldots \ldots \ldots 18 \ldots \ldots \ldots$

Reasoning and Theory ...................... 183

Trust and Prior Belief . . . . . . . . . . . . . . . . . . . 185

The Insubstantial Pageants of Meaning . . . . . . . . . . . 187

Chapter 11. The Writer on the Spot and on the Line .......... 191

The Problems of Spread of Shared Understandings and Action . . 192

Literacy and the Organization of Society. . . . . . . . . . . 193

The Challenges of Learning to Write. . . . . . . . . . . . . 195

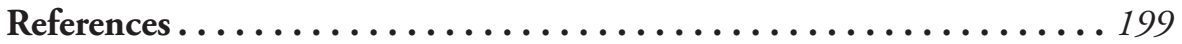





\section{A THEORY OF LITERATE ACTION: LITERATE ACTION VOLUME 2}





\section{INTRODUCTION}

Aristotle set the terms for rhetoric over 2500 years ago. Classical rhetoric established a powerful, useful, and enduring set of concepts for producing and critically evaluating persuasive statements in the public sphere. Its concepts provide means of reflective understanding and choice-making relevant for the class of language productions it arose from-namely high stakes, public, oral performances on matters of deliberative and judicial governance and occasions of commitment to state enterprises. As a consequence of the success of the institutions it reflected on, modern institutions of governance have tended to rely on and replicate the forms of citizenship embodied in classical rhetoric, thereby giving enduring relevance to rhetorical categories.

Yet the world imagined by rhetoric is far from the whole social and communicative world. Even in ancient Greece and Rome, the same agora where rhetoric was established contained discursive worlds of sales and contracts. And when rhetors went home, they engaged in a variety of familial and intimate discourses. All of these would have gained from a reflective understanding and informed choice making, but they were not the subject of rhetorical theorizing. Furthermore, institutions and forms of social participation have expanded greatly in the last two millennia, in large part fostered by the affordances of literacy. The presence of literacy over the last five thousand years has given rise to many new genres, has transformed social life, and has given rise to new forms of social organization dependent on writing as a communicative infrastructure, a repository of knowledge, and as a collection or recorded commitments. Academic work, scientific disciplines, and government bureaucracies are held together by the reading and writing of texts. Only a small part of these texts could conveniently be labeled as persuasive in any traditional sense. Even law (which in the courtroom can be seen as paradigmatic of rhetoric) now is much a matter of libraries, filings, briefs, and case files as it is of dramatic courtroom oratory.

At the end of chapter one of the companion volume, A Rhetoric of Literate Action, I rapidly reviewed the history of rhetorical theory's attempts to address the problematics of writing and produce a workable rhetoric to guide us in navigating the literate world. I concluded there that we still have yet to reconceive rhetoric fundamentally around the problems of written communication rather than around rhetoric's founding concerns of high stakes, agonistic, oral public persuasion.

A further reason to rethink rhetoric is the emergence of social science over the past century, to provide us new understandings of individuals and societies, 
and how individuals interact and participate in societies. The social sciences now provide strong tools to reconceive what it is we accomplish through writing and how we go about accomplishing it. In this volume I explicitly present the conceptual grounds for the theory I propose in terms of major schools of contemporary social scientific thought. Most basically, I draw on sociocultural psychology, phenomenological sociology, and the pragmatic tradition of social science. Based on an account of human sociality and communication that arises at the intersection of these, I consider the kinds of orders embodied in texts and on which texts rely—social, linguistic, textual, and psychological. I particularly attend to the problem of communication across time and space among humans who biologically evolved social and communicative capacities in face-to-face activities. With the emergence of literacy as part of human cultural evolution, new kinds of relations and activities formed that have created structures of participation in larger and more distant organizations, relying on accumulating knowledge and mediated through genre-shaped texts. It is for these activity contexts that individuals must produce texts, mobilizing the resources of language, and it is within these contexts that the texts will have their effect. Near the end of the book, at the end of Chapter 10, I summarize the theoretical path traveled in the book in a way that can also serve to guide us on the way:

1. developmental theories of self and consciousness arising in social interaction saturated with language in order for social creatures to seek life needs and satisfactions;

2. phenomenological sociology, which finds the emergent order of everyday social activity resting on processes of typification and recognizability;

3. pragmatic theories of self and society, seeing self, society, institutions, language, and meaning constantly being transformed to meet human needs;

4. structurational sociology, which sees larger structuring of events and relations emerging interactionally from the local actions and attributions of participants;

5. anthropological and psychological studies of discourse practices as situated, distributed, and mediated;

6. speech act theory, which sees utterances going beyond conveying meaning to making things happen in the social world;

7. theories of discourse as dialogic, situated, and heteroglossic; and

8. a rhetoric oriented to content, purpose, and situation as well as form and style.

While this theory may make some conceptual breaks with the rhetorical tradition in its focus on the problematics of writing and its grounding in contemporary social science, I still draw on many of the founding concepts of 
rhetoric, which are discussed throughout both volumes. More importantly, I maintain a commitment to the practical rhetorical project of providing tools for reflective, strategic use of language. I hope that others will entertain the new concepts offered here as within the rhetorical tradition, but providing a new direction for the way forward as we begin to address the practical needs of composing communications in new media. To do that, however, we must first come to terms with the world of writing which has become infrastructural for modern society, even as modern society is venturing into new digital ways of being.

I have been working on these two volumes in one form or another for a quarter-century, with two early promissory notes sketching early versions of the theory and the need for it (Bazerman 1994a, 2000a). It has been a struggle to tell the theory of these two volumes clearly while still respecting the complexity of writing. To accomplish this, I have made some choices. In order to maintain focus on the underpinnings of the theory proposed, I have not engaged with a full discussion of the rhetorical tradition, but rather have used concepts from the tradition as they are usefully integrated into the theory I propose. Similarly, while there has been extensive contemporary research in writing studies, I have cited such research only insofar as it aids the exposition of the theory, even though much research could be cited in empirical support. I have discussed these findings extensively in my other publications and have aided their dissemination in numerous sites, including the Handbook of Research on Writing (Bazerman, 2008) and several book series I have edited.

This and the companion volume can be read separately. While there is, I hope, consistency across the exposition of practical considerations in the Rhetoric of Literate Action and the theoretical exposition of this Theory of Literate Action, there is no one-to-one correspondence of the chapters, as each book follows its own logic. Nonetheless, some core concepts of the former volume do have fuller expositions in specific chapters of this volume. The issues of spatial and temporal location raised in chapters two and three of the Rhetoric and motivated social action in chapters five and six of the Rhetoric are examined extensively throughout the first seven chapters of this volume, as I present the location and situated action choices within communication at a distance as the fundamental problems of writing. Genre, which helps solve these problems, appears throughout both volumes but has its most explicit treatment in Chapters 2 and 8 of the Rhetoric and Chapters 3, 4, and 10 in this volume. The role and nature of intertextuality discussed in Chapters 4 and 9 of the Rhetoric are the topic of Chapter 10 here. The problem of representation of meaning in Chapter 9 of the Rhetoric, here is addressed in Chapters 9 and 10. The temporal experience of texts discussed in Chapter 10 of the Rhetoric is here theorized in 
Chapter 10. Style presented in Chapter 11 of the Rhetoric is examined from the linguistic perspective in Chapter 8 here. The issues of writing processes and the accompanying emotional and cognitive issues considered in Chapters 10 and 12 of the rhetoric receive theoretical treatment here in Chapters 2, 4, 5, and 11.

As with the companion volume, I am deeply grateful to the many, many people over the years I have learned from, shared ideas with, and worked with as I struggled to make sense of the complexities of writing. Most recently, for their thoughtful reviewing of the latter stages of this manuscript, I thank Anis Bawarshi, Joshua Compton, Christiane Donahue, David Russell, Sandra Thompson, and the anonymous reviewers of the WAC Clearinghouse. Finally, I could not have come to these thoughts on writing without the good fortune of having met a partner over forty years ago who shares the passion and adventure of writing, Shirley Geoklin Lim. 


\section{THE SYMBOLIC ANIMAL AND THE CULTURAL TRANSFORMATION OF NATURE}

\section{WRITING AS LEARNED ACTIVITY}

Writing, as all life is, is activity. When writing, humans are doing things, purposeful things, things that transform themselves, their relation to each other, and their relations to the material world. The reason for inquiring into writing is to understand what we are doing, to learn how to do it, to learn to do it better - and to help others do, learn, understand. Writing is a skilled, invented, learned, historically emerged sociocultural activity—not instinctual, not programmed directly into genes and stimuli-released hormones. Homo sapiens emerged perhaps 200,000 years ago with strong social orientations from prior species and with newly emerged language capacities. About 5000 years ago (Schmandt-Besserat, 1996), however, we found new ways to enact our social and language capacity within a new symbolic environment for us to attend tofostering new skills and capacities to meet new challenges and opportunities. By participating in and through this new symbolic environment we have been able to transform our meanings, relations, identities, and activities. While there are strong arguments to suggest that our general language capacity biologically evolved in dialectic with the development of our means and practices of language and social interaction-that is, nature and culture co-evolved-the introduction of writing has been so recent and its general spread to the great majority of humans only within the last few centuries, that there is no reason to believe that there has been any biological adaptation to favor writing. Writing relies on biological machinery thoroughly in place before literacy, assembled for non-literate purposes—such as our visual discrimination, our hands able to manipulate fine objects, and engage in small operations, and our capacities to use language and other symbols (See Deacon, 1997; Donald, 1991). Since written language is apparently a sociocultural evolution without the benefit of any specific biological evolution selecting for skill in writing, any biological variation in the way we participate in written language, would depend on variation that is not specific to written language, such as variations in eyesight 
or general processing capacities in the pre-frontal cortex, or abilities to imagine and respond to non-present situations.

Writing from its beginnings relied on human invention, an invention that we constantly extend and elaborate, that we learn to do new things with and work with more deeply. Writing is an invention we are still learning to exploit, learning to carry out new activities with. Likewise, any rhetorical theory of writing is a new invention, a means of reflective understanding of the choices to be made in order to extend our abilities to use writing. A rhetorical theory of writing is a bootstrap to do and see more, a way of acting at different levels, incorporating new considerations.

Inventions occur in the course of humans trying to do things-such things as coordinate life in a society, improve agriculture, extend and exercise authority, keep track of property and property transfers, inform others of the great deeds of leaders and forebears, enlist cooperation with the authority of leaders, encourage particular values and attitudes, keep each other amused and cheered, or provide services for which others would provide goods in return. Every time writing has been used and therefore developed through expanding uses, it had functional use within an activity. Even play, which seems so separate from the goal-directed activities of life, enacts human desires and frustrations and explores behaviors, meanings, skills, and tools that seem effective in the lives of others and might become effective in some imagined life of the people at play. Play activities are what they are because of their relation to the more directly goal-bound activities of life. So just as every manifestation of language is an instance of situated language use, so every manifestation of writing is an instance of use of writing by some individuals in some place for some purpose.

Setting about the act of writing requires high focus, intention, and motivation. Even at the physical level, gathering the materials for writing, placing ourselves in a physical environment that makes writing possible, focusing our visual attention on small sign and manipulating our writing tools with fine motor skills require preparation and long skill development. All these preparations require intentionality even when we use convenient electronic devices that we can operate in almost any environment. In the past, when we actually had to buy paper or even prepare parchment, fill our pens, locate a desk apart from the winds of the fields, and form legible characters, the barriers of material and skill preparation were even higher. Material considerations aside, cognitive intention must be high to compose messages to those not physically present, to anticipate difficulties, to organize extended statements, to gather thoughts and facts, to build coherence, and to face the risks making our messages available to be examined later by others. These are not faced lightly and we must have 
strong purposes to motivate us to such inconvenience, physical and mental effort, and risk.

Therefore, a theory of writing must also be founded on a theory of activity, but it must also distinguish itself as a particular form of action, realizing its action in particular ways. I will present writing as a form of mediated, learned activity that carries out social activity at a distance. Writing works through cognitive means that align writer and reader to common perceived locations of symbolic interchange and then carry out specific interactions within that space. In that space the writer offers temporally and spatially organized representations, transformations, and acts in an attempt to influence the cognitive state, disposition, and mental organization of the readers, but which the readers attend, to interpret, evaluate, respond to, use, forget, or remember from their own positions, situations, and interests.

It is in the art of rhetorical writing for the writer to increase the influence or effect of the sort the writer desires on readers. It is in the art of rhetorical reading for each reader to locate, interpret, and evaluate what is being offered from the positions, interests and understanding of the reader, for the reader's purposes. The interchange mediated by writing is complex, potentially making available a cognitive meeting ground in shared representations that is nonetheless entangled with individual differences of location, situation, interests, material conditions, material engagements, knowledge, beliefs, commitments, skills, and motives. Writing - the making of texts — is a form of work aimed at transforming the thought and behavior of others, and thus coordinating relations in the material world, through inscribed language, transmittable through time and space.

\section{ACTIVITY, WORK, AND TRANSFORMATION OF CONSCIOUSNESS}

The theory here is grounded in Marx's view of work as transformative of nature, including the nature of humans. Culture, in turn, consists of the accumulated tools and mediational artifacts we employ in our labor (See Fromm, 1961). Work does not consist only in the reductionist sense of paid work and the accumulation of cash value, a very particular and local historical means of organization of labor. Rather work comprises all we do to make our lives together as social and material creatures in our social and material circumstances. This labor of transforming the conditions of our life in accord with our desires, aspirations, and imagined possibilities, is itself a product of our consciousnesses that arise out of our orientation to our material and social conditions. Our consciousness is directed toward achieving our objects or goals; 
that is, those transformations that we strive for. Our consciousnesses are part of the activity of living and are action directed. Marx, following Hegel but in his own way, presents human consciousness as historically changing, thus giving meaning to the project of phenomenology. Marx sees the history of consciousness tied to our changing forms of labor-that is, the ways in which we transform nature to make it our own, and make it knowable to humans and part of human life.

Rhetoric and writing are deeply implicated in the formation, orientation, and activities of our consciousnesses-as we form much of consciousness through our participation with others through language, and we learn to make meaning (that is states of consciousness in ourselves and others) through these culturally developed mediational tools.' Through language we learn to influence others' consciousnesses, make sense of the consciousnesses around us, and gain tools for the development of our own minds or consciousness. With literacy we have more extended, contemplative, and potentially eclectic resources for the formation of consciousness. Just as we make up our minds in talking through our impulses and ideas, we make up even more elaborate states of mind through the writing of extended texts that also potentially influence the states of consciousness of others, insofar as they attend to those texts as part of some activity of their own, an activity that may be part of a conjoint project with us.

Because we transform our world and ourselves through our labor, and the labor of language is particularly transformative of our consciousnesses and interactions, language work is essential to what we have become as a species and as individuals. Further literate interactions facilitate more sustained engagement of consciousness, are a major means of aggregating and making accessible the historical products of cultural evolution, and are also implicated

1. Marxian critical analysis of language is most often directed at false consciousness, where individuals are interpellated into ideologies serving the interests of others, leaving no room for agency (Althusser, 1970). These forms of critical rhetorical analysis typically consider such issues as the power to control discourse, the interests served by various ideological structures, silencing, and other means of enlisting and coercing people into discursive formations not of their own making and not of their own interests, so as to be deprived of their own linguistic instruments of self-making (Derrida, 1981; Foucault, 1970). Marx however had an agentive view of individuals working within available circumstance and of forming ideals and objects and goals within and from their circumstances, so constantly inventing/creating an ideological sphere of their own making, not necessarily false except insofar as they have been alienated from their own true interests, desires, and concerns. Without alienation language can be seen as a realization of human potential, a realization of ways of being. A Marxian rhetorical analysis of non-alienated language would be phenomenological and ideational, considering the situated forms of self and social realization made possible within circumstances and available linguistic tools. These forms of expression have the potential to serve as fulfillments of the individual and group's impulses towards self-expression and actualization, as Volosinov began to sketch out (1973). 
in the formation of complex modern human institutions which change our relations and attentions and goals. Consequently, any rhetoric and theory of literacy need to be attuned to the history of consciousness and the history of social organization and interaction. Each literate interaction is embedded within particular moments in the changing possibilities of human consciousness and relations.

Although Marx is generally recognized as the primary vehicle of historically evolving consciousness seated in material conditions, and therefore this stance towards language and consciousness is generally associated with socialist political positions, the same perspective was equally present in the founding of western democratic capitalist thought. Adam Smith expressed a similar thought almost a century earlier, when he noted that the knowledge and experience of each person was shaped by the conditions of work (Smith, 1976, 1978). Smith further notes the modes of thought available to each was conditioned by that experience, and further this was differentiated and organized socially and economically through the division of labor and formation of classes. Smith's observation grew out of Locke and Hume's recognition of the individuality of formation of mind out of each person's history of experiences that underlay the set of associations. Smith, as a rhetorician and social theorist, was early on concerned with the difficulty of communication given that we had such individuality of experience and association; he then took that recognition of variety as a resource in building understanding. Only by sympathetic reconstruction of the position of the other and understanding of their situated state of mind could one begin to be persuasive to others (see his Lectures on Rhetoric and Belles Lettres, Smith, 1983), understand human reasoning and knowledge (see his History of Astronomy, Smith, 1980), or begin to act responsibly and morally with relation to others (see his Theory of Moral Sentiments, Smith, 1986).

Smith, along with the other Scottish moralists sees the ground of morality as seeing ourselves as others would see us (as his contemporary Scot Robert Burns put it), even though there are limits to how much anyone could put oneself in another's positions, so that ultimately we are thrown back on our own reconstruction of how others might see it if they knew all that we knew and saw it from our position. Yet it is the generally available patterns of experience that at least provide a beginning of understanding of the range of experiences and positions likely - and thus class, trade, social group, and other large forms of social and economic order can tell us much about the range of experience, thought, and position of individuals in a society. More particular understandings of individuals then grow out of the particulars of their lives. Thus we understand, as best we can and within limits of knowability, each other's minds as historically located within life interests and conditions. This 
is the beginning of communication, social order, and production of humanly useful knowledge. By becoming reflexively aware of these operations of society, Smith argues, we can philosophically order and make improvements on human arrangements. In all these perspectives he is very close to Marx, and together they point to a historical understanding of consciousness constantly emerging in the changing conditions and arrangements of life and the forms of work by which we attempt to meet the necessities of and improve our lives. (See Bazerman, 1993b for a more detailed analysis of Smith's understanding of language and rhetoric).

The rhetorical need to understand ourselves and others to communicate and cooperate locates the consciousness formed by reading and writing even more within social and historical circumstances. Each text comes from a moment in cultural and social history-a history of interactions in pursuit of human life as it is then currently organized, as conceived through the forms of consciousness of writers and readers in their moments. These forms of consciousness are expressed in and through the forms and typifications of language as used in realizable projects in those historical circumstances. Similarly, each utterance is located within the history of each person's life, within located activities within that life, and it is received by equally situated people. For people's consciousnesses to meet over meaning, therefore, some recognizable mediational place must be established in which minds may find a common ground, across time and space. People, to paraphrase Marx (1963), make their own utterances but not in linguistic, historical, and material circumstances of their own making. However, through linguistic invention they are able to create new communicative circumstances at some levels of remove or abstraction or extension from their current immediate circumstances, thereby transforming their own immediate sense of place, sublating or transforming it to be viewed and communicated with from some more idea-lized position. They are also able, therefore, to form new social relations through the mediation of language. As we will explore throughout this work, literate use of language provides more extensive tools for the transformation of circumstances and the institutions that develop on the bases of these texts. Literate use of language also provides greater opportunities for contemplative and reflective understanding of our utterances and more extensive possibilities for the elaboration of consciousness, as well as for the material circulation and persistence of texts. ${ }^{2}$ Thus, the history of

2. The themes of literacy supporting reflection, elaboration, and durability were initially explored by the first generation of theorists of literacy and orality, including Goody (1977), Havelock (1981) and Ong (1982). More technologically determinist versions of this argument have been criticized as "the autonomous model of literacy" by Street (1985), on the grounds that different societies use literacy in different ways, no particular consequence is pre- 
literacy and sites of literate interaction are an important part of the history of consciousness and therefore the transformative work of making human life.

But before considering the transformations of human life and the creation of new locales and situations of interaction accomplished through writing, it is worth examining more deeply the early biological and cultural transformations that were part of developing the language capacity.

\section{BIOLOGICAL AND CULTURAL EVOLUTION}

The view of the effects of language use and literacy presented in this chapter is evolutionary and follows in a Darwinian mode, but sees cultural evolution as an extension of and intertwined with biological evolution. It is only because of the social and cultural nature of humans, the possibility of which is a result of biological evolution, that cultural evolution is possible. Human learning and symbol making allow us to transform our experience of nature and create novel relations to both nature and each other. Learning, symbols, and consciousness also allow the transformation of goals and activity, so that we pursue novel ends (not directly determined by our biological inheritance though indirectly supported by our inherited biologic capacities) with respect to nature and each other, thereby further transforming culture, society, and nature. Finally our ability to create artifacts and employ them as part of our transformative work with each other gives a robustness to cultural evolution and an elaborative complexity to our learning, as each generation grows up into changed material conditions incorporating the new artifacts of the prior generation and the changed social and material practices and relations employing those artifacts. Language and literacy are major elements in this cultural evolution creating artifacts of great power to change consciousness, social relations, and material practice.

Cole (1996), Deacon (1997), and Donald (1991) among others have integrated the literatures of psychology, neural and brain science, anthropology, paleontology, archeology, and biological evolution, to create accounts of the intertwining of biological and cultural evolution. Their accounts suggest that not only did biological evolution set the conditions for cultural evolution but is

determined, and many of the functions attributed to literacy can and are carried out in oral cultures. Nonetheless, not all versions of the transformations of literacy argument require determinism, uniform uptake, or absolute divides. Rather, the needs, desires, and opportunities of societies shape how they will see and use the potential affordances of writing to facilitate and extend prior functions, eventually to establish new modes of social organization and new potentialities of meaning. 
intertwined with it, as earlier forms of sociality and culture proved biologically advantageous setting the stage for primates to become biologically equipped for increasingly complex social interaction and cultural production, in a spiral of cultural-biological change of at least two million years.

Deacon and Donald both tie the development of language to prior developments of symbolic behavior that serve, among other things, to transform social relations. Deacon particularly ties symbolic behavior to the marking of social roles and hierarchies serving to transform the natural order in social constructions that carry organizational weight even when they cannot be recreated at every moment. That is, a mate does not have to be there every moment to announce the attachment if the relationship is memorably defined through ritual and symbolic markings. The symbolic not only copies or represents nature-it transforms it and creates meanings. Deacon's account of how the brain selects, reinforces, and strengthens connections between perceived objects and their symbolic remarking or transformation suggests how the human brain adapted to take advantage of the opportunities offered by the new means of language to elaborate and reorganize social life. This suggests the neural mechanisms by individual as they develop internalize cultural tools, as proposed by Vygotsky (discussed in Chapters 2 and 3.) ${ }^{3}$

\section{THE TRANSFORMED AND EXTENDED HERE AND NOW}

This transformation of perception and meaning facilitated by language marks the here-and-now in new symbolic ways and allows the development of more intricate forms of cooperation and social organization and identity and role within the group-and the ability to operate within larger social groupings. Even more, language facilitates the representation of distant objects

3. Donald emphasizes more the mimetic transformation of episodes as prior to further semantic transformation. This semantic mimesis allows us to give particular meanings to our life, meanings that reshape our perception, behavior, and decision making, transforming and to some degree obscuring our own pre-verbal means of knowing. Deacon, on the other hand, sees us as idiot savants of language, with the expansion of the prefrontal cortex which we then largely organize for symbolic activity and symbolic transformation of preverbal activity. Previously nonsymbolic, unreflective neural activity is then controlled through conscious reflection in language. Both are very close to Vygotsky in seeing language as the means of reflection and in transforming prior forms of cognition. All three see the language capacity as developed phylogenetically and ontogenetically (as species-wide competence develops over many generations and as individual skill develops during a lifetime) in social conditions for social purposes, creating social meanings that become part of enculturation as people learn to work with these terms to mediate their social interactions. 
in the intersubjective here-and-now of talk. That is, an ape when confronting a task may remember a previous moment seen as similar and may search for a tool that is part of that memory. A dog may remember a spot where a bone is buried. But neither can enlist another creature in that memory and in the search for the object. It cannot represent that non-present or non-simultaneous object, cannot call it to the consciousness of another, and thus cannot bring something distant into the current activity, short of somehow bringing the other and placing the distant object in front of them, as ants compel their peers through pheromones to visit the site of food. The fact that a few species have developed elaborate but limited symbolic means to direct the attention of peers to distant objects, such as the honey bee dance, only indicates the great value of having a flexible ways of expressing the nature and location of distant objects and events, and the development of neural means of processing these varied and flexible symbolic representations.

With the development of language, what then becomes considered relevant to the here-and-now is constructed by participants through language. Through talk, one person calls objects and events to mutual attention through symbolic marking. The terms and concepts in which these accounts are cast themselves mark out categories, ontologies, ideologies, perceptions, and perspectives on activities and the world. These terms and concepts put us into symbolic and reflective relation to the world, and this reflective relation is socially shared and confirmed. Just as body markings or adornments mark someone as a mated partner or a tribal chief, so words begin marking out an idea-saturated landscape in a process that has come to be called the social construction of reality (Berger \& Luckmann, 1966). Through talk we become interpellated into each other's accounts of reality, in pursuit of the activities we share. That is, people tell each other things and evoke in others' imaginations objects and events presented as locally relevant, thereby enlisting, orienting, and providing necessary information for the other person(s) to take part in a shared activity.

Others can of course challenge the relevancy or accuracy of any account, or can distance themselves from the activity they are being enlisted into. They can even attempt to negotiate the task and activities by putting forward their own accounts and assembling their own set of relevancies to place before others. They can as well recount the events and relevancies in different terms, so as to make the setting and its assembled context different, thereby making it a different situation. Eighteenth century Scottish philosophers (including Smith, as discussed earlier) in particular noted the role of peoples' accounts of their situations as a means to sympathetic understanding of their positions and the beginning of cooperation and social cohesion. This was a theme picked up by the American pragmatist school of social sciences including Dewey, James, 
Sullivan, and Mead—each of which provided the foundations of developments in education, psychology, philosophy, psychiatry, and sociology (see Chapter 5).

Nonetheless, these representations of the extended world not otherwise visible and pressing in the here-and-now are only brought to attention in the here-and-now of the participants, as perceived relevant to the here-and-now situation. With awareness that society grows out of the action of participants in the local here-and-now constructed by the participants, conversational analysts begin their examination in the micro-interactions of social talk, first of all in the turn-taking system-who gets the floor to assert his or her activities, his or her version of the world and relevancies - and then next in the membership categorization devices that assert the ideologically relevant world invoked by participants. Similarly, the linguistic anthropologist Hanks (1990) sees the deictic system of language as central in constructing the perceivable and attended-to world that participants think and work in, particularly in socially cooperative or socially organized systems. Even more, he (along with a number of other sociolinguists and linguistic anthropologists) sees the very meanings of all terms as indexical, tied to the mutually accepted and constructed hereand-now of the communicative situation; the meaning of words cannot be tied down except within the situation as perceived by the participants (Collins, 2011; Gumperz, 1982, 1992). Thus both linguistic anthropologists and conversational analysts only find meaning in the unfolding interaction which attributes meanings to what has been said, and takes those meanings as given in further actions both linguistically and in the material world acted on. (Clark, 1996 provides a psychologist's version of the same theme.)

\section{NON-SYMBOLIC AND SYMBOLIC COGNITION}

These socially constructed meanings through symbols serve to displace and transform our existing forms of non-symbolic cognition, though they do not erase them entirely. Research on color-coding for example has had two kinds of findings. Most salient is Rosch's (1977) findings that our prototypical colors encoded in language and understood by the users of those many languages tend to be organized around the colors made biologically salient through our visual receptors. Even earlier it had been noted that while culturally encoded colors aid recall of colors, we are able in real time to perceive and match colors for which we have no name. That is, we can immediately perceive in ways that do not rely on symbolic transformation, but without the support of symbols the immediate physical representations fade rapidly. Further we can act in immediate physical and social coordination without symbols. The newborn infant and mother 
coordinate care-giving activities without symbols through mutual recognition of presence and sensory experience and bodily coordination with each other. In our daily life we engage in many non-symbolic acts of motor coordination and instinctive adjustment, too quick for conscious thought or for which we have only weak and pale vocabularies that cannot capture all we experience. Experiences of food often go well beyond the vocabulary of the eater.

As we develop experiences in the human symbol-saturated world, these moments of non-symbolic cognition become limited, often embedded and called upon within complexes of symbolically constructed social realities. A person playing soccer engages with non-symbolic embodied thinking in response to the ball entirely in a private perceptual-motor kind of thinking, but if the player has been coached, even that immediate activity becomes influenced by self-regulatory words. Even more, to organize such individual activities in a game and to focus one's energies on developing these embodied skills over time require a large set of social meanings enacted in language that give reality to the game and establish social value and rewards for participating in the game. This symbolic work establishes the here-and-now of the playing field during the time of action of the game and the times of practice, confirming the camaraderie of players on and off fields, creating meanings for victory and defeat, and establishing the social prestige and economic rewards which have meanings well beyond the time and place of the game.

As our more embodied experiences, actions, and thoughts become enculturated into social frames of meaning offered by available language and other symbolic systems, our very experience becomes transformed, as the taste experience of a trained chef or an oenologist has become transformed by internalization of elaborate systems of taste categorization and knowledge of the components and production of the food and wine. Enculturation of a child is also part of the process of cultural terms becoming salient in monitoring behavior, directing attention and perception, sizing up situations and initiating responsive action. Learning language is part of learning to do things, and using language is part of entering into the available and desirable social activities in which language is implicated. The child's request for "more" or "no more" facilitates feeding as well as creates a child who learns to use language to assert needs, desires, and preferences. Learning to tell jokes is an extension of sociality and bonding among family and associates; it also forms a new kind of activity that could not be carried out without language.

Yet, even while symbols refigure and transform much of our experience, symbols are still created within embodied motives and experience through talk or other significative physical action to another human to whom we are orienting. Language emerges as part of human beings in co-presence, attending 
to each other, and doing things together. Speech, though symbolic, engages large parts of our biology in its production and the entire body's orientation and participation in a situation - cries for help as one is struggling in ocean waves or the coordination of a work task through rhythmic instructions. Equally, though more subtly, people engaged in as abstract a symbolic activity as arguing over the truth of a proposition can enact all the passions and postures of oppositionthough transformed through the etiquettes of literate civility.

Literate symbolic activity is no less a total body experience, though often it is not accomplished in the presence of other humans, and although much of the external forms of behavior fade away as the distanced world of the text overtake the orientation of one's nervous system and one's attention turns away from the immediate world surrounding the arm chair. The history of writing and reading is filled with traditional embodiment. Many early texts were memory aids for spoken events to be re-enacted, whether by a nuntio reading aloud the words of a king to a distant governor, or the script of a speech to be memorized and performed, or the words of a legal code invocable in court, or the words of a god to be regularly read aloud as part of liturgy and study.

Even without oral performance of the read text, literacy is still associated with the vocal apparatus of speech. Whatever the first act of silent reading may be (whether as some folklore has it by Alexander the Great wanting to keep a message secret from his troops or Ambrose witnessed by Augustine in fourth century Milan, or some other unrecorded occasion), it clearly was not a general practice until the time of monasteries. Until the medieval script of Carolingian miniscule, reading Roman scripts required reconstruction of words from a text without spaces to aid word recognition. Similarly consonant-only scripts (Abjads) such as Hebrew, Aramaic, and Arabic, require reconstruction of the oral word from memory. Reading aloud to children and children learning to read aloud themselves remain crucial parts of literacy education-with a particular emphasis on reading with expression or feeling as an indication that one gets the meaning of the words. Disability such as deafness or blindness which interferes with the association of written and spoken words creates special challenges in learning to read and write (Albertini, 2008).

Animating meaning of words in one's mind is an act of animating oneself, as one's imagination, emotions, and anticipations become engaged in creating meaning as a writer or reader. No matter how much the activity is carried out internally in seeming bodily repose, various parts of the nervous system are influenced by even the most calming text. Moreover, even the most civilized readers or writers find moments when anger or pleasure arises, when they can't keep their bodies from tensing or they burst out in laughter. If you watch younger readers and writers who have not yet learned to hold most of the literate action 
inward you can read the somaticism of literacy in the postures and movements. And if you watch writer's struggling with their words, getting up for cups of coffee or muttering to themselves, you get some indication of the insuppressible engagement of the neural system in the production and processing of text.

\section{LANGUAGE AS SITUATED, EMBODIED UTTERANCE}

This view of the capacity to use language places meaning at the center of the language competence, makes the value of language inseparable from meaning, and places meaning in the minds, motives, and actions of people. Even Saussure recognizes the centrality of meaning for understanding language when he defines the sign as a unity of the signifier and signified. Yet for analytic purposes Saussure distinguishes langue (a system of language) from parole (the motivated uses of language in situations), and then makes langue the object of linguistic study. While this move has been successful in creating an extensive linguistics, it ultimately is misleading about language, for language exists only in the utterance, and any attempt to abstract a language apart from its uses obscures the concrete functioning of language in evoking meaning as well as those complex processes by which we come to understand each other (this point is elaborated in Chapters 7 and 8 of this volume). Saussure's related move of distinguishing the historical change of language from an abstracted system of contemporary langue (the diachronic/synchronic split) obscures the historical emergence of language as a regulated system (through social negotiation and through such inventions as schools and grammar books).

The view I adopt here places societal and individual language development as part and parcel of our other activities in providing a new tool for their realization, thereby transforming them. Understanding language in this view requires understanding the activities it is part of and the meaning systems that evolve as part of the language-using activities. It also suggests plasticity of the brain and language processing as the person's language and brain develop as part of social participation mediated through language. Further, individuality (of experience, social situations, momentary needs, and motives) results in individuality of each person's experience of language and developing language competence, even as all individuals may orient towards the quasi-stabilized socially available forms of language they encounter. Neurologically and evolutionary plausible accounts present syntax itself being the consequence of the growth of semantic knowledge (Elman et al., 1996), with syntactic learning of the available ordering and morphology of the sentence predictably occurring only when vocabulary reaches a certain size, of around two hundred words- 
so that far from language being characterized and led by a pre-programmed syntactic competence, that syntactic competence is a situational way of ordering and using an extended vocabulary within activity-based utterances.

Wherever the debate may go over the nature and origin of language and associated human capacities, the formation of a semantic relation to the world around one is clearly formed within the social interaction, the activity contexts where one begins to exchange symbols with each other as part of conjoint activities, ultimately emerging as something like conversation. Further, language develops to fit the use of people in real circumstances in relation to material contexts. As a number of commentators have pointed out the problem of reference cannot be overcome by an autonomous language within itself, but must be attached to perceptions of concrete objects (Goodwin, 1994).

Whatever parameters of language are set by our neural apparatus, language evolves to fit these constraints and opportunities. If it is biologically hard for us to work with a linguistic structure, or a means of pronunciation, or a conceptual structure, we will search out a linguistic means that makes communication easier to produce and process. Each child in learning the language transforms it to meet his or her needs and capacities. Similarly, as material conditions and social projects change such that new terms are needed, new activities and operations need to be indexed, or new complex structures need to be elaborated to carry out the actions, linguistic means will be invented to facilitate these needs. If old linguistic practices are no longer intelligible because of change in social activities (the vanishing of oral epic or qualitative verse) or material circumstances (the need to coordinate several people in the capture and harnessing of wild horses) the particular linguistic means associated with them will wane or become transformed to have new meanings useful in the evolving social world. Political speeches, for example, may become transformed to rely on the linguistic techniques and tropes of mass media entertainment and advertising instead of the heroic cadences of epic.

The particular interactions, activities, symbolic resources, interactive and material challenges in which people learn to use language are inscribed within the neural system of the growing child, as human brains strengthen and pare neural connections over the life of the person (Gogtay et al., 2004; Petanjek et al., 2011). Further the acceleration of myelination particularly within left hemisphere prefrontal cortex during adolescence (Paus et al., 1999) is especially associated with many of the intellectual functions of language that are introduced in writing instruction at this age in some social and educational settings. Our brains form in interaction with the material, social, and symbolic environments. Our minds grow to be able to use the tools of language, and more recently writing, just as they grow in learning to manipulate the legs and 
hands or the attention and coordination of the eyes. Thus the child in a sense builds itself, or neurologically adapts itself, in relation to the current moment of culture, society, and economy that it finds itself in.

As Smith, Hegel, and Marx suggested, consciousness indeed changes throughout history in relation to the material, social, economic, and cultural arrangements, as our minds form to cope with the world we are born into. Modern neuroscience is starting to show us indeed how this changing consciousness forms itself into the very organization of the brain. It is at this point that Marx and Darwin meet.

\section{LANGUAGE, LITERACY, AND COGNITIVE DEVELOPMENT}

When we first do start to discover the power of symbolic communication, much of our social interaction and material perception has already developed along particular pathways which our language then enters into-to elaborate, work with, and to transform it, but always in interaction. Thus the child who is color-blind has extra work to accomplish and arcane adjustments to make in order to learn to speak in the standard language of colors suggested by the society (Sacks, 1996). The child who has a playful interaction with parents is likely to develop a playful and creative linguistic repertoire to elaborate that play. The child whose parents allow their gaze and attention to be influenced by the child's gaze and attention is likely then to be able to build linguistic techniques of sharing experience and entering into conjoint activity that include the child's state of being and interests, in contrast to a child who can only gain the attention of the parent by learning to align themselves with the parent's gaze. On the other hand, in some cases the development of language provides means for the realization of activities that were not possible through previous means-such as playing rule-governed instead of ad hoc games. The child's learning of the word no is well-known as providing great power to selfdefinition and choice making-though the cultural opportunities to explore and extend the applicability and range of this aspect of consciousness and social behavior is very much shaped by the cultural practices and behaviors of the people surrounding the child. That is indeed why in western nations there has been a cultural campaign in recent decades to valorize the child's learning of no as an important developmental task instead of treating it as a sign of willfulness and disobedience.

Although emergent literacy experiences, such as playing with paper and talking about books may happen as early as talk initiates, actual visual recognition of words and meaningful inscription usually happen a few years 
later, when many of the fundamental pathways of behavior and language use are already well established. This means that literacy sits as an add-on to an already developed cognitive architecture, which it can draw on from the beginning. The presence of books around the house, the integration of literate activities into daily acts, the way in which this occurs are important to the ways in which literacy becomes deeply embedded within the child's notion of the life the child is developing into. How a child relates to the world indexed in the text is dependent on the earliest and most fundamental ontologies and relationships the child establishes with the world and with other people (Heath, 1983). Children with wide experiences with many people of many views and personalities can more readily recognize a range of views represented in reading, while those who experience only an adult-authoritative rule-governed world may find it harder to explore the range of worlds texts have to offer. Further, if literacy and books are part of daily life, children will be better prepared to see the power of literacy and to adapt it to multiple circumstances, while those who experience literacy only within the school walls for formal educational activities will not immediately see the purposes of reading and writing beyond the fulfillment of school requirements. Later experiences may extend their experience, and transform their understanding of use and literacy, but this means moving beyond patterns that have already taken hold.

Much can be said for the kinds of social bond developed around the hearth, or the forms of social interaction and physical health fostered by a youth spent wandering the woods or on the baseball diamond. These are possibilities of life world and deep values to be expressed through human development. But insofar as the world appears to children to be permeated with books or computers which offer attractive sites of interaction, then an early literate environment is likely to have deep transformative effect in children's organizations of their minds so as to make sense of and interact through these symbolic media. If we wish to promote these as mediating tools appropriate to the adult way of life of our society, the early and deeper the participation, the more pervasively the full range of the child's experience is likely to integrate and be transformed by these symbolic communicative opportunities.

The rest of this volume examines theory and research that help us understand more fully the way language and literacy mediate the development of our minds, experience of life, social activity, social goals, and social organization. The first half presents three social science traditions that emerged in the late nineteenth and early twentieth centuries-Russian sociocultural psychology, European phenomenological sociology, and North American pragmatism. The latter half of the volume, building on these three traditions and enlisting more recent social thought, examines the kinds of order we create, participate in, and use to 
make meaning in our writing: social order, interactional order, linguistic order, temporal order, and intertextual order. These various orders converge in genre as a recognizable invocation of these multiple orders and recognizable place that each of our utterances take within them to assert our unique, situationally relevant meanings. In the companion volume A Rhetoric of Literate Action, directed towards our immediate practical needs as writers, genre appears front and center as it focuses the location of our work as writers. Here, however, in order to show why genre is such an important concept for writing, we must first examine the underlying conditions of human cognition, sociality, activity, and communication that pose the need for recognizable and familiar locations for literate interchange and then how that recognizable location organizes the work that happens in that place. This broader theoretical groundwork for genre supports a more comprehensive understanding of genre recognition as a human communicative process. Thus genre lurks everywhere underneath this volume, to regularly poke its head above the surface (particularly in Chapters 3 and 4), but only to take topical centrality in the final three chapters. 



\section{SYMBOLIC SELVES IN SOCIETY: VYGOTSKY ON LANGUAGE AND FORMATION OF THE SOCIAL MIND}

Lev Semyonovich Vygotsky's examination of the processes and effects of symbolic participation on the formation of the human mind provides insight into how the symbolic organization of human consciousness is part of our integration into socially shared forms of expression, meaning, and activity. Vygotsky's work, carried out in the early years of the Soviet Union, was neglected in the West and elements were suppressed under Stalin, but since the 1960s the power and significance of his work has been increasingly evident both in Russia and the West. There have been numerous explications and interpretations of his work, which I will not attempt to reproduce here (See, for examples, Daniels, 1996; Daniels, Wertsch \& Cole, 2007; Kozulin, 1990; Van der Veer, 2007; Van der Veer \& Valsiner, 1991; Veresev, 1999; Wertsch, 1985). Rather, I will explore how his approach to psychology connects individual cognition and affect to social role, activity, and consciousness as a social being — and therefore one's reflective engagement with the world, particularly as that engagement is mediated by language and writing.

The work of Vygotsky will provide a meeting point for much of the work I will describe in the ensuing chapters, but not because that work follows directly from Vygotsky. Only a distinct part of it was done with any significant awareness of Vygotsky or even working from common sources. But rather Vygotsky's interdisciplinary style and the particulars of his ideas invite the synthesis of social, psychological, linguistic, and historical concerns. I have over many years found his work to be ever fresh because it is so open-despite many aspects of the work undeveloped, others barely gestured at, and others inaccurate about particulars we have later discovered as we have gained more data about sequences of child development and the cognitive capacities of other animals. Nonetheless, his ideas allow us to move back from the largest issue of society, culture, and history back into the complexity of human selves, thoughts, feeling, and development as we engage with the world. From the point of view of teaching, learning, and development, his theory respects students' motivated and autonomous selves, yet recognizes how deeply those selves are saturated with social interactions and resources and how those selves grow into the possibilities of the worlds available to them. Similarly, from the point of view of writing, his theory provides a way 
of understanding the formation of deep interiority and individuality of meaning within a world of communicative interaction and social exigency, and it provides a means for accounting for meaning that arises in forms not yet attached to words and then becomes transformed as it takes shape in meaningful languagewithout resorting to ill-defined wells of thought entirely separate from language.

\section{LINGUISTIC STRUCTURE AND LITERARY AFFECT: VYGOTSKY'S CATHARSIS}

In the turmoil of Russia between revolutions, Vygotsky simultaneously attended two universities, gaining a degree in law following a traditional curriculum at Moscow State University while simultaneously earning a degree in literature and aesthetics at the alternative Shanyavskii People's University (Wertsch, 1985, p. 6). Then taking a position teaching language and literature in a high school in his home village of Gomel, during the early years of the revolution he became an active member of cultural life, publishing widely on cultural matters (Van der Veer \& Valsiner, 1991; Veresev, 1999). Vygotsky became interested in the psychological effects of literary works, in particular the relationship between the literary structure of the work and affective states aroused in the reader. Even as an undergraduate student of literature he saw a crisis in aesthetics torn on one side by a purely individualist psychology of perceptions and imagination of the audience and on the other by an idealist philosophy that considered the "nature of the soul" and not the material facts of reader response. When he returned for an advanced degree in psychology in the early 1920s, he continued to work on the same problem, arguing in his dissertation on the Psychology of Art for a more situated and embodied view of the response evoked by texts that are historically situated within ideological structures of their time. In this work (Vygotsky, 1971), he himself does not provide any concrete socio-historic analysis; in fact, at this point he sees the sociological and historical study of ideology as distinct from psychology. He, nonetheless, does pursue detailed analyses of how texts can evoke particular states of emotion, and thus mediate experience. While he was later to see ideology as bearing on the material conditions that shape psychological response, for the time being he was content to consider the audience located in the act of reading the text or witnessing a play as the right level of analysis to understand affect. Indeed throughout his career he was to maintain focus on the individual acting within a limited situation, usually mediated by specific available artifacts.

In the primary example of a psychology of art, a detailed analysis of Bunin's story "A Gentle Breath," Vygotsky directs our attention toward the contradictions 
built in the story between the dismal facts of life of a young woman and the light-hearted, though misguided, spirit that carries her attractively through life. The narrative rearrangement and selection of events and the movement through the consciousnesses of several characters brings to poignancy the attractive delusions that lie behind the woman's dismal fate. It is in the affective poignancy of the tension that the story achieves its aesthetic power.

Similarly, in considering Hamlet (reworked from an earlier school essay on the subject) he looks for the logic of Hamlet's wavering and erratic behavior not in a psychological explanation of the protagonist's character, but in an aesthetic of motive and digression that places the audience in a state of emotional tension and contradiction. The words, the logos, of the play do not present a coherent logic of an argument but rather comprise a device to arouse the audience's emotion. He points to an additional level of affect that arises when we look on or reflect on this character who seems so to tease our emotions and not resolve them: we are left in a state of puzzlement. Most critics pursue this puzzlement directly by trying to find an answer to the "Hamlet problem," the explanation of Hamlet's behavior. Vygotsky sees these critics as responding to an external logic imposed on the play's events rather than understanding it. He would rather we ended where the play ends, overwhelmed with the contradictions and conflicts that resolve only in a tumble of conflicting and absurd actions.

Vygotsky considers his wedding of formalist, structural accounts of texts with an analysis of the affective states of the audience evoked by these structures as a theory of catharsis_- "a discharge of nervous energy" resolving conflicting affect aroused by the work of art. Consciousness is not directly dictated by the ideological contents of texts, but rather consciousness is activated and placed in troubled spaces. Consciousness and the affect that infuses it arise in the problematic tensions the mind struggles with; thus he finds a way to link consciousness with the material structures of language and the materiality of the cognizing being, yet nonetheless granting the individual a personal place of responsive consciousness which is not a mysterious other arising from in an ineffable core of individuality. Although he is concerned with response, he is careful to note that since we do not know the minds and affects of readers and writers we can only attempt to understand the emotion-evoking devices in the texts. We do not necessarily feel what Shakespeare felt, or Bunin, or what any onlooker now or in the intervening centuries may have felt, yet if the play or story does affect us, it is by the devices in the artistic artifact designed to arouse our embodied emotions.

In this early work, we can already see Vygotsky's interest in states of consciousness as influenced by textual devices; he sees language mediating experiences. He sees his psychology of art as a materialist form of interpretation 
rather than an idealistic one, a realistic psychologically serious correction of the purely intellectualized symbolic analysis of the formalists. For Vygotsky the symbolic constructs of ideas serve to arouse bodily sensation of emotion rather than simply evoking more ideas. He includes introspective observations of his own breathing rates in reading the story, and was soon, in his first psychological experiments, to measure breathing rates of subjects reading the Bunin story (Van der Veer, 2007).

At about the same time Vygotsky completed the Psychology of Art as his Ph.D. dissertation in psychology in 1925, he was delivering his first papers at psychological conferences, arguing for the need to study consciousness, but with the behaviorist caveat that language was itself not to be interpreted as a direct and reliable introspective report of consciousness, but rather as part of the process of reactions involving consciousness (Vygotsky 1925, 1999). That is, language and utterance were to be considered as behaviors in relationship to consciousness, rather than as the contents of consciousness. Just as he had considered the lack of attention to the affective states aroused by art as the cause of a crisis in aesthetics, he viewed the lack of attention to states of consciousness in relation to behavior the cause of the crisis in psychology. Further he argued for practice, the application of psychology to real world problems, as the necessary motive and test of psychological theory and research. That is, the human needs confronted in application call into question abstracted theory and unrealistic findings by re-embedding research into the complex and concrete processes of life, at the same time as people engaged in practice need strong theory and research to guide their work. The result of the interaction of research and practice will be stronger, more useful, and more concretely grounded theory. Vygotsky's own thinking was deeply influenced by his foray into practice, in the area of defectology (a term jarring to contemporary sensitivity about stigmatization), the field we would now call disability studies or special education. He was deeply engaged in practical work in this field from 1924 until 1930, when the institutional base of his fieldwork collapsed in the face of political decisions (Veresev, 1999, p. 127).

\section{GOALS, OBSTACLES, AND EMPOWERMENT: VYGOTSKY'S ADLER}

Vygotsky's attention to consciousness and the tensions within it helped him cast a new perspective on the fate and struggles of the disabled in attempting to live their lives. Rather than seeing the psychology of the disabled as just a matter of what capacities they had and didn't have, LSV paid attention to the way in which people reacted to their limited abilities and the kinds of social positions 
they were cast into by their disability. At this point in his intellectual journey, he was also particularly attracted to the work and thought of Alfred Adler who was concerned with people's desires to reach their goals and to overcome obstacles or frustrations in reaching those goals. Adler considered people as active agents: you could not understand people's behavior only on the basis of biology and history. Rather you had to know what they wanted and then what they understood they needed to do or overcome in order to get what they wanted. Adler as well posited a general developmental desire of all individuals to gain increasing power over situations, particularly in comparison to others who might be viewed as potential competitors, models, comparators, or obstacles. This is especially true of children who seem relatively more powerless than other people around them, but are biologically, neurologically, and cognitively in a period of development - with the promise of them becoming more capable and more able to equal or best those around them. This modeling and competition has a strong sociological component, as the developing child draws the range of desirable goals, opportunities, possible means of action, and possible competences from what she sees around her. The child develops into the social relations and socioculturally formed situations and roles around her (Adler, 1907).

These issues of desire for competence and power over one's life are particularly poignant in relation to the disabled who find themselves in a world designed by and for the typically abled, and a social world that additionally stigmatizes and creates limited roles for the disabled, as Vygotsky began noticing. While the disabled may directly attempt to compensate for or overcome their disability by other means (whether by appliance like the blind man's stick, increased attentiveness and reliance on other faculties, or social cooperation), Vygotsky noted they also needed to overcome the kinds of social roles they were cast into by others-whether as objects of scorn, pity, or paternalism, all of which limited and framed the possibilities of action, relations, and situations they could participate in. Further the disabled need to overcome the difficulties of a world designed for the convenience of the abled - a world that puts curbs on roads, places steps at the entrance of buildings, and organizes space and activities through visual cues such as street signs and traffic lights (Vygotsky. 1993).

In line with these observations about social roles and material obstacles, Vygotsky recognized that the desire for power to participate competently was not driven so much by a generalized sense of desire or lack, but more drawn by the concrete opportunities available in one's society. People set their goals and possibilities from the available choices, and thus frustrations occur when people cannot be part of what is going on around them. This is very much in line with what sociologists would consider reference group behavior and social modeling (Merton, 1968b) and what Bourdieu (1993) would consider the social field of action. 
While Vygotsky first held more closely to an Adlerian view of direct supercompensation for perceived inferiority which would lead the disabled person to try to overachieve in just those areas they found themselves most challenged, he moved to a broader view of the restructuring of mind, personality, and organism around the conditions and opportunities the person found themselves in. Thus over-compensation (finding alternative pathways to the same goals that others have) became not the only possible dynamic, but rather a reorganization of the self to deal with the circumstances one found oneself in. As more recent neuroscience has suggested this can be seen even at the most basic level of neurocognitive organization developing in the young child. As will be discussed in the next chapter, Vygotsky had an interest in the neurological foundations of what he was noting and with his colleague Luria began to study medical neurology. Luria was to become one of the pioneers of modern neurology in which he was to take what he called a "romantic" view (Luria, 1979) not just as biological facts, but of the organization of a personality coping with circumstances and neurological conditions.

Some of these means of reorganization could entail organizing new tools into consciousness, as the blind person learns to gain visual information through a stick, or through collaboration with the seeing-eye dog, or through alliance with others with a different range of skills. Here we can start to see the growth of Vygotsky's awareness of how much the mind grows in relation to mediating tools and relations. These extensions of the self he saw as becoming part of the organization of the self. This went beyond his earlier recognitions in the psychology of art that cultural artifacts such as poems can create temporary states of consciousness that then activate bodily sensations or reactions. Here the tools and relationships are actively taken on and employed in pursuit of one's desires and life, empowering, but also organizing consciousness and personality. One learns not only how to attend to and control the stick or dog, one learns to sense through them, to perceive the world through them, and to think through knowledge gained via these media. We just don't pass through a poem for a temporary sensation; insofar as that artifact becomes a long term mediating tool in our life, we come to live through it, making it part of our fundamental orientation, activity, means of sensing, and acting.

\section{COGNITIVE TOOLS}

All these issues poignantly and strikingly evident in relation to the disabled provided Vygotsky a way to rethink the development of the more typically abled. In the early 1920's at the beginning of his career as a psychologist while 
he was still learning the field he had written a volume on paedology (excerpted in Van der Veer \& Valsiner, 1994). Toward the end of the decade he returned to issues of development and education, in a series of publications that were to come to stand for his cultural historical theory and his distinctive contribution. This work has been extensively summarized and is available in English through several translations of Thought and Language, and the collection Mind in Society and in a less refined version in Studies on the History of Behavior: Ape, Primitive, and Child (Vygotsky \& Luria, 1993). My discussion of this work will emphasize particular lines growing out of the earlier work and pointing towards its relevance for symbolic communication, cultural evolution, formation of the cognition in relation to social communication, and particularly literacy.

In this more fully developed theory he took an interest in cognitive tools that extended or externalized our thought, allowing us to carry on symbolic activity outside of ourselves: the knot tied around the finger to stimulate memory, the abacus to keep track of and manipulate numbers, the South American quipo used to record messages and history, and ultimately language, spoken and written. To investigate how we used these external symbolic tools to carry out cognitive tasks, he conducted experiments using the technique he called double stimulation, in which the original task stimulus was supplemented by a secondary set of stimuli which the experimental subjects could use to help carry out the primary task. For example, in the forbidden colors task, children were asked a series of questions about the color of objects, but in their answers told they could not mention two colors nor could they repeat a color used to answer a previous question. When they were given a deck of color cards to assist them in the task, children of age five to six years either did not use these cards, or if they did, the cards distracted them from the primary task. Children of eight or nine years old used the cards to identify the color names that were used and forbidden or to identify the colors still available for answers, but they were inconsistent (or not fully disciplined) in using them. Children of ten to thirteen years used the cards in a consistent, disciplined strategy and made few errors. Adults made few errors whether or not the cards were available, as they were able to keep track mentally of the disallowed and allowable color names (Vygotsky, 1978, p. 41).

From such experiments Vygotsky identified what he called the second stimulus system, the set of signs which we learned to use to regulate our behavior. The process by which these signs were internalized was observed through watching how young children seemed to talk to themselves. Earlier such private speech had been thought as simply egocentric, but Vygotsky noted how the utterances coordinated with the tasks that the child was carrying out as well as imitated prior conversations with others as together they carried out 
similar tasks. That is, the child was drawing on remembered social resources to reenact privately an activity coordinated or directed through language. Just as an adult had attempted to direct the child's behavior through language, now the child directed his or her own attention and activity through similar language, though increasingly fragmented over time as the fuller forms of language no longer seemed necessary for self-direction. With time the reliance on the external device of language seemed to vanish as the child could carry out the task without spoken self-regulation. Vygotsky hypothesized that the language turned inward and became the basis of symbolic thought, changing its form as it became internalized.

By such mechanisms we can see how prior experiences of language become formative elements in the development of individual thought - not by direct importation of a language symbol or ideological system, but because the child first interacts with the language in the course of activity and then redeploys that language as part of self-regulation in tasks including his or her own interactions with others. That is, language becomes the child's own as he or she uses it in particular circumstances of life, fulfilling individual needs at the moment. Out of this process the individual creates personal meanings. In use, language becomes transformed into meanings which influence perceptions and actions and which become the bases of novel communications with others, so that the individual populates those words with his or her own intentions, as Bakhtin was to write later (Bakhtin, 1981). Personal use of language, however, is saved from solipsism because when it is used again to communicate with others, the need to be understood by others disciplines it towards social norms of meaning, as George Herbert Mead was pointing out on another continent a few years before (Mead, 1913).

While Vygotsky considered the expression of personal meaning within the social sphere in the last chapter of Thought and Language, his psychological interest in the development of mind was more directed toward how language moved inward as signs to direct self-regulation and self-organization. Because of this interest he distinguished signs as different from tools, because he saw language as most significant in regulating the self as signs became internalized. Following this interest and characterization of language as sign rather than tool (See Vygotsky, 1978, 19-30), he was able to develop a rich system of selfdirected, self-monitored consciousness based on the internalization of socially received language which comes to transform the self. Because of Vygotsky's concern with the development of the self, he does not develop as fully the ways in which our mind continually is transformed in more mature social interactions, how we come to develop our impulses and thoughts by externalizing them and thereby become socially committed to them in our identities and actions, 
and how our participation changes the social field. Nonetheless, much of his evidence is drawn from how people deploy their symbolic resources within tasks. Further, his analysis of a child's learning scientific (or schooled or socially disciplined) concepts examines how children's spontaneous concepts come to be transformed by organized interaction with received knowledge coming from a history of cultural and communal testing, validation, organization and reasoning (Vygotsky, 1986, Chapter 6). However, he tends to treat that scientific knowledge as fairly fixed-neither reinvigorated nor transformed by new participation. He did not yet make the link between individual personal development and larger cultural development, though he does recognize culture itself as resulting from a human history of invention.

Yet even from Vygotsky's limited social and cultural account of knowledge formation, we can see the importance of the particular symbolic systems and activities one participates in and internalizes in shaping the kinds of tasks one can carry out and in the organization of one's mind in relation to the tools and tasks. The historical accomplishment of a culture is made available to each new child as he or she finds meaning and use in the available tools and artifacts which can be redeployed for the child's own purposes in the social settings and activities he or she finds themselves in. The discursive and activity landscape the child perceives provides an opportunity space for the child's development and participation. While Vygotsky largely seems to be thinking only of broad sociohistorical cultural movements as shaping the available forms of cognitive growth available to the child, he seems at times to be aware of the multiplicity of socially organized positions people find themselves in, as he considers for example the role of stigmatization in shaping the interactions of the disabled and thereby channeling cognitive growth along particular paths (Vygotsky, 1993).

More fully, however, we may consider the effect of having available specific kinds of cognitive tools associated with particular groups or professions, in carrying out specialized tasks. People who engage in the legal tasks of corporate law in the United States in the early twenty-first century carry out substantially different tasks and thinking than biochemists working for those very same corporations. Their tasks require them to do different things; the cognitive tools they must learn and think with support different kinds of work and are themselves differently organized; the kinds of symbolic interactions with their interlocutors are significantly different; and they organize their own thinking in different ways in relation to these tasks, tools, and relations. Koranic scholars in sixteenth century Baghdad, court poets in Elizabethan England, preColombian Mayan scribes each follow their own line of cognitive development in relation to tasks, tools, and relations they participate in. We do not need to look at the highest ends of literacy in radically different circumstances to see the 
impact of differentiation of cognitive development, but need only to consider the way young children's engagement with ball sports or drawing or word games will focus their attention, modes of thinking, and self-reinforcing social relationships within others engaged in those activities and associated social arrangements. These experiences, activities, and relationships shape sets of skills and cognitive orientations that initiate trajectories of competences throughout their life. Within the worlds of literacy we can see the differing consequences among the children who have a taste for fiction, a taste for political biographies, or a taste for books about zoology.

Developed cultural practices and forms, identifiable as distinctive genres, discourses, disciplinary languages and tasks-the typified practices that characterize the differentiation of our social and cultural worlds—can be seen in Vygotskian terms as particular sites of activity deploying particular cognitive tools and supporting different lines of psychological development. Individuals in learning and internalizing these cultural forms, use them to regulate their own perception, thought and ultimately participation with others. Medical doctors, for example, within the typical settings, events and communicative forms of consulting office, hospital and professional meetings, use their medical knowledge to examine, diagnose, and administer treatment to patients who may have little understanding of medicine.

\section{SECOND ORDER SYMBOL SYSTEMS AND CONSCIOUSNESS DEVELOPMENT}

Alphabetic writing, Vygotsky notes, is a second order symbol system that offers a visual sign for the spoken word, rather than directly representing a perceivable or an imaginable object. The words in speech provide a symbolic representation of the events and objects discussed at only one remove, except for reflexive second order speech that references words as language ("What do you call this tree?"). Writing, however, creates a second order representation. That is, written words are symbols of spoken words. This of course is most pronounced in alphabetic languages where written words record the sound of a word, which then has an attributable referent or meaning. However, even pictographic or ideographic or rebus languages use the symbols to represent the word (despite some possible graphic association with the objects or events referred to). Pictographs are highly stylized and selected around a limited and typified vocabulary - that is what distinguishes them from simple drawing. They then can be used to create hybrid and elaborate complexes, again with standardized word associations rather than open-ended complexes of non- 
linguistic associations. Even where the immediate language is not spoken, but visual, as in a sign language, the written representation again provides a somewhat durable second order representation of the immediate transient word.

Writing's second order nature abstracts writing out of the immediacy of perceived, unfolding experience, and creates a need to reconstruct some indexical relation to an embodied reality, beyond that required in spoken language which can typically draw on the material context of utterance to ground its indexicality. That is, what one talks about is often visible or can be pointed toward or gestured about, but in writing it is typically harder to tell what the text is talking about. Further, the writing only indexes the spoken language and thus all meaning must be indicated through the relation to the spoken, with the spoken further stripped of its material context. Thus the relation of the written to the spoken presents a problem almost as soon as a writing system develops beyond the most concretely iconographic. This is perhaps the reason that one of the earliest forms of knowledge to emerge in most written languages is some version of linguistics (Bazerman \& Rogers, 2008a). Writing transforms more immediate, situationally and viscerally prompted use of language into an independent linguistic object that can be more easily and reflectively manipulated and managed, and is therefore more easily and more pressingly studied, for purposes of strategic and precise effectiveness.

Writing as a means of reflection and self-regulation can transform the local in relation to distant situations; even more, writing can create new places for symbolic participation that transform the participants and provide new venues for self- and mind-making in interaction with other literate participants. Some forms of writing do stand in immediate relationship to on-going embodied experiences, such as the shopping list that guides mall behavior or the series of instructions that regulates the preparation of pre-packaged food. But other forms of writing enact social relations and activities that operate at a reflective distance to our daily activities, such as reporting and commenting on political events, contemplating principles of effective leadership style, or playing with the possibilities of imagined romance. Through these second order reflections on more immediate experience, created in a second order medium already abstracted from more immediate symbolic practice, writing interaction can enter in and through consciousness, influencing the writer and readers in ways that may not be quickly forgotten or dispensed with. Unlike spoken language where words are inspired or compelled by the immediately unfolding events and then leave no trace to prompt or constrain memory, writing leaves an external mark for us to look on later, transforming our attitudes and perceptions of the utterance.

As we get drawn into literate interactions we recognize and seek out the textual places where they take place. For some these sites of literate interaction 
became major sites of identity and interaction, drawing on increasingly intense cognitive and affective engagement, thereby shaping their literate minds and personalities, in accordance with the domain of texts and interactions they find most engaging. Because of the visibility of the linguistic artifact and the removal from daily time and space, such spaces are conducive to creating reflective distances and stances towards material events, the literate events one participates in as a reader and writer, and the texts that mediate those events. Writing thereby facilitates interiority as we commune more with other literates than with the people around us. With interiority we orient toward the interaction played out in our mental construction and reconstruction of the meaning of our texts and the texts of others. Additionally, writing facilitates interpretation, criticism, irony, and other stances that put us at a questioning distance from our interactions. But interiority and questioning also foster creative behavior, allowing us to return to our embodied world with fresh perspectives, ideas, and resources to address life problems and challenges.

\section{INTERACTION AND SELF-REGULATION: INFLUENCING OTHERS AND INFLUENCING THE SELF}

Understanding language as both interactive and self-regulatory suggests an often-confusing dialectic about language. Theorists of language and particularly writing often see language as deeply personal, formative of character and expression, tied to our deepest experience and thoughts. Vygotsky notes how we build our thinking and transform our experiences (including the kinds of presymbolic experiences and eidetic memory and thinking available to children prior to development of language) through our growing linguistic experience, and he himself in the final chapter of Thought and Language has deeply poetic reflections on language as fragments of our innermost thoughts. On the other hand, others see language and writing as rhetorical and interactive, shaped by social purpose and effect, little driven by anything like an essential expressive self. Vygotsky also suggests such perspectives when considering how the parent uses language to help the child solve a puzzle or the blind gain through social means information not available through eyesight. Finally there are those who suggest that language is a meaningful system that exists outside any of the participants or particular utterances or usages, whether that language consists of stable resources and rules or that language is a locally produced, ad hoc artifactual construction. These three perspectives align with three major approaches to writing-the expressive, the rhetorical, and the linguistic. 
Vygotsky gives us a way of understanding how all of these are operative simultaneously as we develop cognitively through social participation, using the available language purposefully. Language is simultaneously within, between, and outside people. Writers need to look externally to the communicative forms, to the organized relations with others, and the ad hoc communicative and rhetorical problems of the moment; and internally to the self, organized and attentive to the evolving discursive situation in order to develop ideas, communicative intentions, and meanings.

We can see how these issues come together in considering perhaps the most well-known of Vygotskian concepts - the Zone of Proximal Development (often called ZPD). In his writings he articulates the concept most clearly in relation to assessing a child's capacity for learning. He states the most important thing to measure is not what the child can do by him or herself (say, in the traditional paper and pencil IQ or achievement test) but in measuring those things that the child can do with assistance of an adult or more skilled peer. This identifies the area of learning a child can engage in leading to development (Vygotsky, 1978, Chapter 6). This ZPD identifies activities where students can enter into novel or challenging collaborations, guided or regulated by the speech or other actions of the more skilled other-speech and actions that can then go from interpersonal regulation to intrapersonal regulation. In this way the child can learn new practices, principles, concepts, and activities which later he or she may be able to carry on by him or herself and ultimately internalize within his or her cognitive repertoire. Further, at some point the elements learned within the ZPD reorganize and coalesce into a new functional system, changing the relations and functions of the previously acquired parts, reorganizing perception, reasoning, and activities. This transformation to a new form of thinking which reorganizes previous ones constitutes development, in contrast to learning. For this reason Vygotsky says learning leads development (Vygotsky, 1986). This process of reorganization based on conceptual development (in Hegelian terms called sublation or aufhebung) provides a way that both genres and mentorship can induct one into specialized forms of perception, reasoning, and practice, such as those associated with scientific and academic reasoning, as well as professional practice (See Bazerman 2009, 2012).

The more skilled participant in ZPD interactions has already internalized the disciplined functional system that constitutes expertise in the activity. This disciplined functional system provides structure to both partners' contributions, making available to the less knowledgeable partner hints about a different form of consciousness available for perception, reasoning, and action. While the student at first may hear and even heed the comments of the adult or more skilled partner, these are at first only taken as specific pieces of guidance or 
information. At the moment of development, however, the learner comes to see events, activities, or relations from the perspective of the more skilled partner, and the learner reorganizes his or her way of functioning and thinking: consciousness has been raised. The ZPD can, in theory, identify both what is next to be learned and the depth or extension of what can be learned (that is, how far with help the learner may reach beyond him or herself and still participate in comprehensible activity). Further, awareness of the learner's developmental challenges within the ZPD can attune the more skilled partner to providing the kind of support that may be needed to maintain that learner's participation. Even more, the skilled collaborator can become attuned to the learner's changing states of awareness, perception, and conceptual grasp (that is, forms of consciousness) and can recognize whether and when learners have made developmental leaps - that is, whether the learners have internalized the higher mode of thinking. Teachers regularly talk about when students show such moments of insight, or "when the lights go on." Such moments are often accompanied by changes in bodily posture, composure, and facial expression.

Writers regularly use the support of cultural tools of genre, of the ideas and information of others, the challenge presented by others' ideas, as well as the constraints of the task at hand to learn how to create the text, which in turn may bring about a change of personal consciousness. The pressures of the social situation and availability of cultural resources help writers to extend beyond what they already have thought, said, or written. Writing under the pressure of new thoughts composed for the situation out of words and ideas from within and around the writer can seem a deeply felt personal expression of the self at the same time as it contributes to social identity and agency, articulating the writer's self onto the social stage-a self-creating act. As writers draw on the common resources of language available to all and familiar to the readers, they become the writer's words, words meaningful to the writer. As the challenge of the interaction stretches the writer, he or she may also reinvent aspects of the language-seeking new words, phrases and metaphors, combining genres, and forming new concepts. Further, the organizational and argumentative challenges of texts that extend over paragraphs, pages, or volumes, can stretch the writer to reorganize thoughts and knowledge. Additionally, the devices of exposing textual organization (like outlines, section headings, and transitional statements) may provide ways to think through organizational problems in composition and revision, creating new coherences in reasoning. The process of writing, using common resources, leads writers to make up or compose their minds, sometimes in ways that bring new thoughts to the social sphere of discussion.

For this dynamic of linguistic, cognitive, and social learning and development to occur, enriching the social and personal and linguistic resources, the task 
must be neither so humdrum and familiar that repeating familiar formulas is sufficient nor so difficult to be beyond the writer's comprehension, articulation, and participation. The situation, support of others (perhaps teachers, coaches, or editors), and resources in the culture and language must be sufficient for the writer to maintain goals and directed activity, while still being able to think new thoughts and write new things. If the writer is asked to address something beyond what he or she can even inchoately make sense of, the learning and creation collapses, and the writer either gives up or reinterprets the task in more familiar terms.

These textual artifacts once produced are commitments of the self: phrases the writer has worked through and terms for private purposes and social effect. The textual artifacts are also potential spaces of meaning for others. Writer's words can populate other people's minds with thoughts and associations, can provide new things for them to consider, and new ways to rearrange and reorganize what was already available to them. Or writer's words can simply in a new context remind them and reinforce thoughts and emotions they have already held. The text may present a forced march of logic and evidence for readers or it may open up large areas of speculation and association, tapping into the readers' own concerns and meanings. In that or any other event, the text acts as a potential support and extension of their own thought. But just as for the writer, for the reader also the task and words must be meaningfulthat is, readers must be able to attribute meaning to the signs, viewing them as neither too trivial nor too difficult to attend to and enlisting them into concerns that hold their attention. A text that works to project the writer's meanings into the reader's mental space in a sense then acts as a zone of proximal development for the reader-a space of symbolic exchange, a space of participation that activates behavior, sensation, thought, bodily emotional response, and ultimately new ways of seeing issues and selves. In this process we can see echoes of Vygotsky's earliest observations from the psychology of art on the cathartic effect of literature, where he recognized that the textual structure in evoking aesthetic response gives release to latent tensions within the reader created by the sequence of textual meanings.

\section{PLACES OF PLAY, SELF-ARTICULATION, AND DEVELOPMENT}

Vygotsky's theory of aesthetic catharsis also has its echoes in his later theory of play (Vygotsky 1978, chapter 7), in that both literature and play to him set in motion frustrations or unfulfilled motives that are in tension with one's circumstances or other motives. Vygotsky particularly notes that 
play is driven by desires for development and empowerment that cannot be yet realized in worldly activity (that is, world-transforming work), often because of the child's lack of developments, skill, capacities, or social role. In Vygotsky's characterization of the motives for play we see the influence of his work with the disabled and his Adlerian recognition of the motive to gain specific forms of empowerment to participate in the opportunities of the world. Children play parents or teachers or drivers of automobiles trying on those roles not available to them in life. Children may also play at being themselves if they want to mitigate the consequences of their actions, or to explore the regulations or expectations the self-consciously conceived role seems to impose. LSV cites the case of two sisters, aged five and seven, who proposed to each other that they play sisters, and in so doing invoked rules as to how they thought sisters ought to behave toward each other (Vygotsky 1978, pp. 94-95). The play involves establishing a set of "as if " rules that define game obligations and become guides for regulation of the other and self in the game. Insofar as the child becomes engaged in the game, the child becomes committed to the rules, activities, motives, and moves of the game. More developed games in fact have motives built into the rules, such as "The goal of this game is to place the ball in the opponent's net by various legal maneuvers. " Further, satisfactions are gained through one's participation in the game, which take one beyond the motives that first drew one into the game.

We can see literature, the arts, and other forms of entertainment as particular places of play, each of which create their own organizations of activity and consciousness that provide place for enacting frustrations, desires, tensions, or other emotions transferred from other spheres of life where they cannot be directly enacted. In the course of play there is not only a release, but a reinvention of the self, developing into new possibilities of being that seemed blocked at first in other domains. These new possibilities of being can then be resources brought from the play domain into non-play situations. These resources can include enhanced individual skills, confidence, and reworked motives, but also the invention of new concepts, ideas, and actions that provide useful tools in other domains or that provide a perspective on other domains transforming conceptualization of activities. Thus we have the continuing critical roles literature, art, and humor have played on society and individual lives. We often see new ideas of social and material possibility tried out, envisioned and communicated through literature, as in socially projective novels of George Elliot or the worlds of science fiction. Or we have unpleasant and socially unrecognized realities portrayed under the playful cover of art, as in late nineteenth century realist literature. In another vein of social transformation in play, we can see the communal cohesion forged over a sports team sometimes mobilized to civic or corporate ends. And we 
see with adults the constant trying on of meaning of life and events through fictions, as well as fantasies of what might be.

Art and entertainment as well become their own disciplines which individuals affiliate with and in which they develop identities, modes of thought and feeling, perceptions, and ways of life. These disciplines and social domains become sites of transformative work and take on economic and institutional presence in the form of industries (such as book industries, sports industries, theme park industries) and socially supported cultural organizations (such as theaters and schools) which serve the fantasy, projective, and developmental needs of large publics and become major parts of the cultural landscape, supporting modes of being and forms of consciousness.

Yet the role of play and imagination, and writing's role in creating it, extends beyond the more overtly recognizable imaginative and playful genres of art. Writing is often produced in situations at some distance from where it is communicatively presented - that is we can work at our own desk long before it is presented to readers, or as part of a collaborative team weeks before a report must be presented to a group of managers. We have time to play around with possibilities, represent alternative realities and plans, organize and reorganize our textual goals and plans, interpret and reinterpret data. We can try out alternative strategies in the face of intractable arguments and resistant audiences. This playing around with the possibilities of our textual creation means that the process of writing allows us to explore different possibilities of meanings we can project into the social world. Indeed many of the disciplines of knowledge and theory formulation have extensive play spaces for speculations and hypotheses based on the exploratory possibility of "what if" an idea were true or useful. Hypotheses and speculations born in the "what if" mode can become the motivation for gathering evidence, doing experiments, or engaging in other modes of inquiry. If the speculation turns out to be persuasive, it can turn into the knowledge, inventions, and projects of the future, transforming the shared life of society.

Although Vygotsky's approach to communication may suggest that talk and writing may begin in immediate social and material needs of the individual and community, it also offers possibilities of writing transforming consciousnesses, knowledge, and society. A realistic understanding of the role of play in life and the activity systems built around play, leads us to a more extensive view of writing in our world, which helps explain why some forms of writing are associated with extending human imagination, feeling, and perception. Many of the forms of writing people may think of as mundane have that same transformative effect, whether to develop a school curriculum, or to project a corporate financial reorganization, or to develop a rehabilitation plan for a released prisoner. 


\section{ACTIVE SOCIAL SYMBOLIC SELVES: VYGOTSKIAN TRADITIONS}

Vygotsky's fertile starting place for understanding the formation of individual conscious within social activities mediated by the culturally available tools gave rise in Russia to two direct lines of work of rather different character, developing different potentials within Vygotsky's work. One associated with his student and collaborator A. N. Leont'ev elaborated the idea of individual and group activity. The other associated with his other major student and collaborator A. R. Luria pursued the development of individual consciousness within the interaction of neurobiology, language development, and functional production of behavior.

Each of these traditions brings an important perspective to issues of writing though neither addresses writing as directly as Vygotsky did. Though Leont'ev does not consider writing or even language much, beyond his recognition that language mediates activities and provides a vehicle for social learning, his work extends and elaborates the notion of activity and its relation to larger systems of social organization. His framework, particularly as elaborated by Engeström, helps us articulate the ways in which writing dynamically mediates communally organized activities. Though Luria specifically focuses on spoken language, he provides ways of thinking about the interaction of language and brain within dynamic activity that have consequences for literate production and receptionwriting and reading. Luria's work suggests the deepest mechanisms by which we absorb and use language-mechanisms which have continuing currency within cognitive neuroscience, a field he is recognized as pioneering. These mechanisms have consequences for how we look on our own language formation and interpretation processes, including writing and reading, and therefore how we reflexively manage them. He gives us means for extending Vygotsky's analysis of consciousness as acts of agency incorporating and building on our linguistic experiences, in relation to our material and biological conditions. While Luria several times identifies writing as beyond his scope of interest by suggesting that writing opens up entirely new domains and dynamics of consciousness because of its removal from immediate circumstances and its particularly close bond with inner speech (Luria, 1959, 1969, 1970, 1976), his analysis of spoken language and consciousness provides an important basis for understanding how further structures of consciousness can be formed to deal with the removal of communication from immediate circumstances. 


\section{ACTIVITY, OBJECT, AFFECT, AND SOCIAL SYSTEM: LEONT'EV}

Alexander N. Leont'ev's line of activity theory attends to the activity of the individual and group, which gives focus and meaning to cognition (Leont'ev, 1978). Conscious orientation to an activity distinguishes impulse from irritation. Cognition is motivated by desire and impulse that fastens upon an object - that is, a concrete thing or state of affairs one wishes to bring into being. The realization of this object forms the activity one is engaged in. Because the activity arises out of fundamental impulses, it is saturated with affect and desire; it is the very expression of what one wants to have and to be and to do.

This impulse to bring something material into being dialectically raises an emergent consciousness or cognitive awareness of what one desires and how to go about obtaining it. This state of heightened and directed consciousness oriented to specific ends makes one particularly receptive to perceptions, information, relationships, and other intimations of things in one's environment that are perceived as somehow relevant to that end. One's awareness of these relevancies shapes one's perception of the situation and one's opportunities within which one may frame specific actions that pursue the activity. These instrumental actions may be at some distance from the initial impulse and the affective drive; they may be more planned, reasoned, and distant, with perhaps lessened affect. They are more workmanlike. So an impulse to prepare an exquisite meal for a friend (perhaps saturated with complex socially and culturally shaped identities and desires) may lead one (at this historical moment in cultural taste and economic distribution of goods) to contemplate and plan a menu with awareness of what hints one has about the friend's taste and range of gustatory experience, available new fashions in food that one may have read about, currently available produce, and a dozen other things that might appear relevant in light of this task. One may even start writing down menus and shopping lists. One goes shopping, cleans the kitchen, checks the cookbook, sets the table, chops the garlic, and undertakes many other actions. While each of these actions are imbued with motives that have set one in motion, they have a consciously planned aspect requiring a more instrumental mind set, perhaps affectively surrounded with pride in one's workmanlike efficiency and competence.

In the course of these consciously planned and consciously monitored actions, one employs many habitual behaviors or operations that one needs hardly think about, such as how to form the letters and spell the words in making the shopping list. While in chopping an onion one may need to attend to the particular shape of the onion and the way the outer skin is or is not pulling off, yet the holding of the knife is likely to take little of one's attention. 
The distinction Leont'ev makes among activities, acts, and operations is a key to the conscious attention and affective load demanded by various components of activity. Activities arise from impulses that take shape and are realized through the activity and thus carry the deepest weighting of motive and affect. While the object realizes the emergence of impulse into action and crystallizes one's mental impulses, it may not be fully known and monitored consciously nor is it necessarily open to complete reflective understanding. The object may be more of an emergent phenomenon, only coming into conscious awareness as it coalesces around a knowable project.

The middle level of actions is what we are most aware of and reflect upon, so as to carry them out in the most effective and efficient way. They can be creative in the pursuit of goals, drawing on resources we are only dimly aware of, but as we draw these resources and creative means of accomplishment we become to some degree aware of what we are doing and how our actions are chosen to carry out our intentions. By implication, we may also say, though not explicitly stated by Leont'ev, that the emotions that attend actions are correspondingly more distant and reflective-somewhat separated from the original motive and aware of how well we are doing.

Operations - those tasks that are so familiar and routinized within one's neural system that one can do them without conscious thought, carry little creativity beyond immediate adjustment to the local circumstances-the placement of the chopping block and our fingers as we cut. We are likely to have little attitude or awareness of what we are doing, and the emotions, if any, will be such as the comfort of doing the familiar or the tedium of repeated actions that have become distant from their motivation. Nonetheless, under the right circumstances, operations can come to our attention, as when we notice the knife getting a bit too close to our fingers and we readjust.

These categories of activity, action and operation, are fluid ways of differentiating motivating, focal, and peripheral attention that can change from person to person, event to event, moment to moment. Learning to transcribe the alphabet may well be the primary activity of a young child extending the limits of motor skills, perception, and conscious attention; writing letters may encompass the child's total orientation to a situation. Later, the transcribing of a letter may be an action in spelling a word, with the recording of a cherished word defining the main activity. Both transcription and spelling later will likely become thoroughly operationalized, as the child attends to creating a meaning or making an impression. In the pursuit of goals we may carry out many levels of work with different levels of intention and complex relations of superordination and subordination. In each case of writing we need to unpack the work in relationship to the complex of events and cognitive acts. Often bringing the 
textual object into being is the realization of the activity-a realization that one does not fully grasp until one has realized it as a material object and as a cognitive commitment. This phenomenon has given rise to the many statements of the sort "how do I know what I think until I write it." Sometimes we may not fully realize the total meaning of a text until long after we have written it, perhaps months or years. Nonetheless, despite not comprehending the full implications of what we have written, we can understand each of our sentences and carry out the larger structures of our text in a workmanlike way. Recognizing the appropriateness, timing, and techniques of each of the actions of text-building helps us realize vaguely perceived intentions with some sense of craft, efficiency, and effectiveness. It is just the total object we have made that escapes our full comprehension. In a sense we are learning, structured by the work of text making, but we have not yet developed sufficiently to comprehend what we have written. Our externalized meaning making has not yet crystallized into an internalized set of structured relations that would make the text fully and immediately transparent.

On other occasions, however, the text to be written is easily anticipatable and can be produced entirely in a workmanlike manner, with no surprises about what we have made. Perhaps when we write an email to colleagues to arrange a committee meeting, the email text and meaning is fully predictable. In that case, perhaps, the activity is only emergent in the work of the committee-we do not quite know what the committee will end up proposing; nonetheless, we seem motivated to get there.

To identify just those activities that present the greatest challenges at the moment, but are most driven by the desire to develop our capacities, identity, or mode of being, Leont'ev (1981) elaborates Vygotsky's (1967) concept of leading activity, particularly with respect to school settings. The leading activity identifies, within the zone of proximal development, the activity which captures the imagination of students most, and which they are working most centrally on mastering. Individually or in a group, we might say this is the thing that we are trying to work out as we engage in the activity, the particular way we are working towards expanding (in Engeström's 1987 formulation).

Leont'ev, from a materialist perspective, was particularly interested in activity as an external working out of innerly-driven impulses. Even mental activity he sees originally situated and driven, no matter how distantly, by some material object in the world, though embedded in cultural history and social practice. He would dissolve the mind-body distinction by seeing mind as an embodied capacity we have developed to be better able to cope with a material world. But mind is not reducible to body, for consciousness having developed then influences the embodied behaviors; mind brings objects into being through activity. 
The development of mind and its realization of impulses occur not just in the material circumstances of individual lives, but in the social life of the commune. Leont'ev points to the regularized social activity systems that give meaning, value, and intelligible familiarity to the activities of individuals. $\mathrm{He}$ cites the example of the paleolithic hunt of mastodons, where the activity of one group of people is to be beaters, making noise and shaking foliage. The activity of these people seems senseless as a way to capture creatures, which they are most clearly scaring away. The noise making only makes sense in relation to the activities of other people, such as those who build corrals and those who guard and close the corral (Leont'ev, 1981, pp. 210 - 213). Activities emerge in groups, and actions are negotiated and assigned to individuals, employing their separate capacities. Operations also occur on the group and individual level, as people's response to each other's signals in coordinating the hunt becomes as routinized as the technique of hitting the drum for the individual. People learn to form goals and activities in relation to the activities of others as they emerge historically in stable and anticipatable forms that allow people to organize work in ways that coordinate with the work of others. The sense arises within the system in which individual takes part, getting meaning from participation in larger collective activity.

The developed functional systems of actions and operations, group and individual, that regularly pursue repeated activities express regularized orders of behavior and activity organization that in turn comprise a social order. A functional system perspective provides a basis for seeing how individual acts of writing and reading, shaped within the regularities of genre, participate in larger social systems of activity, rising above individual acts into carrying out larger social endeavors. Thus we have a link here between the inner contents of consciousness of people engaged in acts of reading and writing, a phenomenology of literacy, and the largest social orders of activity within which we organize our lives.

\section{COMPLEX ACTIVITY SYSTEMS: ENGESTRÖM}

Yrjo Engeström, starting with problems in the coordination of work in organizational settings, has elaborated this idea of socially formed activity systems, functionally organized to carry out particular activities through conjoint work. The work and coordination of various participants to produce a shared object is aided and organized, materially and socially, by division of labor, rules of work and participation, and the tools available to carry out the work. To help analyze the operations (in the Leont'evian sense of unreflective 
automatic practice) of any organization, Engeström has developed a heuristic diagram, which makes visible and open to reflective readjustment processes that have been so long in place they are not usually consciously attended to (Engeström, 1987). Engeström's model is based on Vygotsky's triangle which interposes consciousness in the relation between stimulus and response, or subject and object.

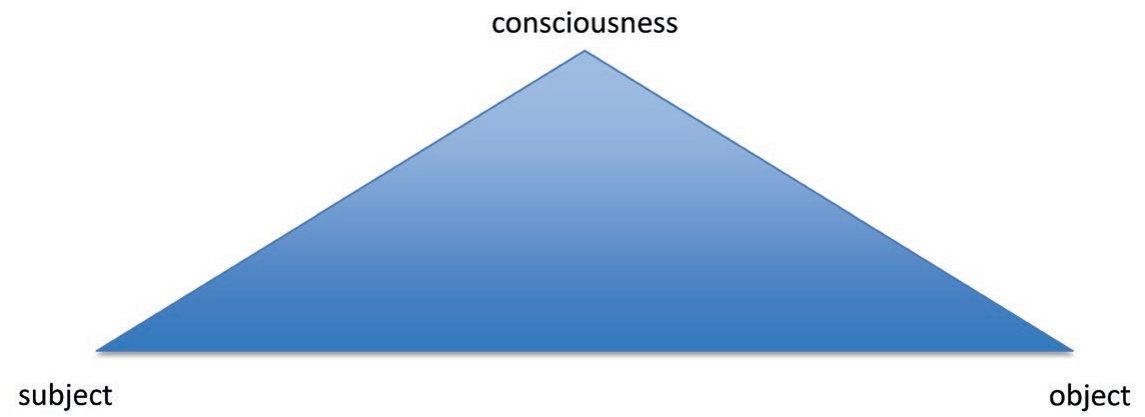

Engeström, following Leont'ev elaborates consciousness as communally formed in shared activities, refiguring the triangle accordingly-the individual working in relation to a community in the functional pursuit of a communal goal or object.

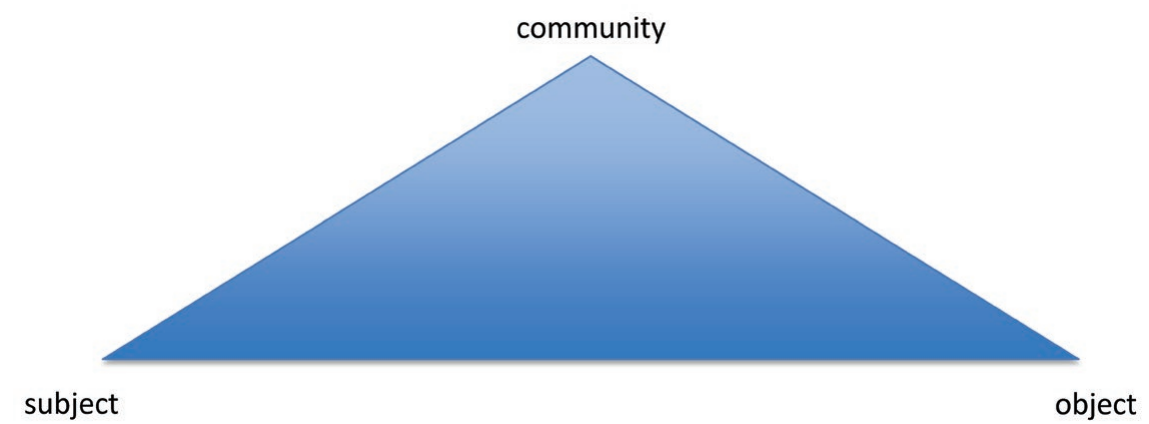

The subject's relationship to the community is shaped historically by the rules that identify roles, responsibilities, transgressions, expectations, rewards, penalties, exchange arrangements, etc. The subject's relationship to the object is mediated by the cultural tools (created through a history of social interactions) by which the object is produced. And the community's relationship to the object is mediated through a division of labor which both distributes and aggregates the total work in production. 


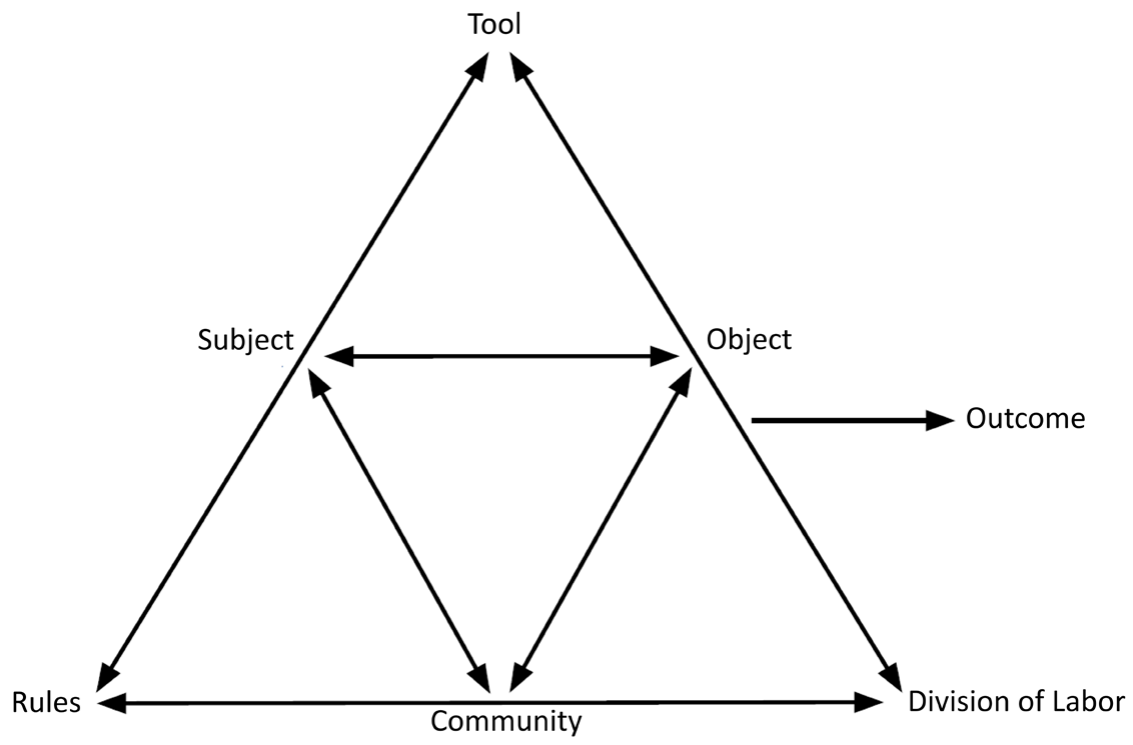

This heuristic helps parse the factors usefully brought into consciousness for intelligent reflective choice-making in what Engeström calls stage III activity theory. Stage I he considers the unreflective interposition of consciousness within a stimulus response of an individual, as investigated by Vygotsky. Stage II activity theory is the placing of individual consciousness within communal activity, but without reflective understanding of the activity system as a whole or one's place within it. Stage III brings to reflective consciousness the social activity system, so as to allow one's reflective adjustment of the system and one's actions within the system.

In any particular case these arrangements are historically emerged both in the larger pattern and the local instantiation. Hospitals, courts, schools have long histories that establish large patterns of arrangements, but each hospital and perhaps each ward, each court, each school and each classroom have developed their own particular set of tools, rules, and division of labor in the formation of local community. Further these are constantly changing in relation to problems, contingencies, opportunities, changing resources, change of personnel, new tools, and so on. So an analysis of any given organization could examine the historical process of emergence of the system to understand the forces the current arrangements respond to, the operations of the current system, and the impulses to change the system.

Engeström has been particularly interested in historically emerged contradictions within activity systems that act as forces to bring about reflection and change. That is, insofar as organizations operate by ingrained and historically 
emerged habits that do not seem to have any sticking points, they have little motive to change or even to look upon their operations, except as routinely monitored in the systematic operations. We might take this as an analogue to Kuhnian normal science, where puzzles are solved, but only as paradigmatic or made typical and habitualized in the system (Kuhn, 1962). So the management of a paper clip manufacturing company would likely monitor sales, inventory, supplies, number of employees, and the like to adjust the level of operations, but they might not contemplate changing the way of doing business, replacing the machinery, changing the product line, or any other element that might reorganize the activity system in a serious way. Only when a contradiction in the system arises - such as workers being unable or unwilling to follow the work rules, or when the machinery breaks down and cannot be repaired according to operating procedures, or when new machinery no longer demands the same division of labor, or when markets shrink for the product—are participants in the organization likely to problematize and rearrange the practice in a more satisfactory way. It is at these moments of emerged contradiction and tension that organizations become smarter, or learn by expanding their reflective awareness of their operations (looking on them as actions), perhaps even reconceptualizing and reorganizing their fundamental object and activity.

Engeström has carried out a number of intervention studies to assist organizations (or activity systems) to learn by expanding their awareness of their operations, and thereby rearrange their world to carry out their functions more effectively or even to adopt more powerful functions. He has had all workers within a hospital analyze their own activity position within their organization, using his heuristic triangle as a guide to consider for example, who provides them the tools, who authorizes the tool purchase and distribution, who sets rules and who monitors them, who sets the distribution of labor, to whom are these various procedures are accountable, and so on (Engeström, 1987, 1993). These questions allow participants to reflect on how the system operates and whether adjustments may be made. Tensions exist in the organization if, for example, nurses must request equipment and supplies from a supplies office which is accountable for holding costs down by a financial office, but they must take their work orders from doctors who demand certain procedures requiring supplies be administered, at the same time the nurses are driven by their perception of the object of patient care. Such discoordination leads to multiple contradictions that call for reflective understanding and resolution. Engeström has also studied instances where individuals who are empowered to initiate reflective actions (such as judges who can revise the rules of procedure in their own courts) spontaneously note some difficulty in procedures and engage various participants (opposing lawyers, social workers, other officers of the 
court) in problem solving to come to novel or flexible arrangements (Engeström et al., 1997). These reflective activities focus attention on the renegotiation of practice within the activity system.

Writing occurs within historically emerged, but constantly changing, circumstances and arrangements. Writing also makes information and textual objects visible for reflective contemplation, opening possibilities of noticing and resolving of contradictions. An important functional element of most activity systems involved with writing is to bring new information or viewpoints into some kind of group contemplation, information sharing, or coordination of perspective. Thus writing regularly offers opportunities to attend to contradictions and tensions, resolving them through wise choice-making in what to include, how to represent and reason with the inscribed material, what stances to take toward the material and readers-all of which goes in to deciding which words to include and how to put them together. The act of writing also usually affords time for thought, the ability to look on and revise earlier plans and revisions, and distance from the place of text circulation-all heightening the opportunities for reflection and process monitoring. Writers and readers, therefore, are regularly in a position of "learning by expanding" (to use Engeström's 1987 term), meeting new challenges of texts and gaining some sense of the contexts or systems within which their reading and writing operates. This opportunity, however, is not always taken and contradictions can remain hidden, often by unthinking adherence to long-standing conventions and practices. Engeström's heuristic in such circumstances can help the writer identify and address the activity contexts it contributes to, and how the text may be brought into greater coordination and effectiveness, perhaps even resolving tensions within the system.

Engeström has pursued another aspect of Vygotskian thought as elaborated by Leont'ev-the emotional attachment we have in the realization of our objects. Objects engage our committed effort to bring something new in the world, fulfilling our needs and desires. In particular, Engeström \& Escalante (1995) have studied the systemic contradictions that may arise from the different emotional attachments people have to an object. In studying an electronic vending kiosk as produced by an entrepreneurial company, as actually used by consumers and as considered by other workers at the site of use, Engeström \& Escalante found that participants had different and conflicting sets of motives, attitudes and emotions. These conflicts ultimately were part of the failure of the device in becoming a regular consumer tool. The producers of the sales kiosk were deeply attached to the technology they had developed under government contract to test for long-term adoption by the post office, for it was the realization of their designs, plans, and action. They, like most 
makers of technology, were in love with what they had made and assumed others would share those sentiments. Consumers, however, only using the machine in the course of other daily activities, assessed the machine from a different perspective, and found it frustrating to operate. The postal workers at the office, whose good will and cooperation could help support consumer use and aid the adoption of the system, saw in the kiosk a disruption of their orderly activities, a reorganization of the distribution of labor, and ultimately a threat to employment. They became antipathetic. Such systemic understanding of emotions is applicable in thinking about the attachment or disengagement people feel for texts they produce and use. Deep attachment is often necessary both for the production and the effortful recreation of complex meanings, but writers' strong attachment to their words are not always matched by the emotional stance and commitment of their readers. The success of a text is dependent on how use of the text contributes to the readers' objects and their engagement with the text.

\section{WRITTEN GENRES IN ACTIVITY SYSTEMS}

If we conceive of each act of writing as a reflective participation in an activity system, then we can see how each act of writing is an historically embedded act of coordinating with others. In these acts of literacy our focus of attention, our objects and goals, may be various but they are directed towards human systems of communication and activity. A writer may be obsessed with developing the narrative technique of an unreliable narrator as a distinguishing characteristic of her fictions or may be most interested in tapping her own depths of subconscious. On the other hand, the writer may be primarily concerned with selling a product or asserting new scientific findings. But in all cases these various literate activities, actions, and operationalized skills only make sense within socially organized systems - whether of literary entertainment or commerce or scientific knowledge production.

Genres are designed for social action, designed to bring about changed material states in the world, transforming our social and material scenes of existence and being. Thus the genres within which people frame their utterances can be seen as also being vehicles for participation in historically emerged activity systems and their ongoing maintenance. By learning to write in the typified forms available at one's time and social place, one learns not only means of participation but the very motives and objects one might have, as Miller (1984) pointed out. Genre-conceived as the form discursive action takesis part of the larger social activity structures within which action takes place. 
Insofar as those social structures are discursively constituted and maintained by the circulation of discourse, the genres themselves are major constituents of that social activity structure, and every individual's use of those forms carries those systems forward. Insofar as individuals orient to those structures as the sites of their actions, and thus find their objects, goals and motives by participation within those social activity systems, their very forms of action emerge as meaningful. Genre-shaped utterances themselves become then vehicles of the production, reproduction, and evolution of the systems within which the genres are meaningful.

My investigations of the emergence of the experimental report in science (Bazerman, 1988, 1991) found that the activity of trying to assert what one has seen-in order to create an empirical account of the material world—required people to learn to argue for the validity, accuracy, and meaning of their claims within the emerging social space of scientific correspondence, societies, and journals. The particular characteristics and dynamics of journal publication provided rhetorical challenges in terms of the publicness of the audience, the enduringness of the text, and the temporal sequence and pacing of articles and responses (contrasted on one side with spontaneous on-the-spot oral response in a small group and on the other with the appearance of books years apart). The typical features of the emergent and evolving form of the experimental report represented rhetorical solutions to the problem of asserting one's findings within such a structured and contentious field. This activity was carried out with great passion and commitment by a number of the early modern natural philosophers such as Isaac Newton and Joseph Priestley, who themselves were major rhetorical innovators and influences in shaping the genre. The normative rules, roles, tools of investigation, production of journals, the positioning of scientists with respect to other contemporary socio-cultural entities, and other aspects of the social and activity structure of science evolved simultaneously with the discursive forms of participation-with major consequences for how knowledge was produced, what forms it appeared in, and what counted as knowledge.

Devitt (1991), similarly, was able to identify the activity of working tax accountants with the production of a set of genres of tax letters that sat in particular relation to the tax code and the client's financial records. Each of the letter types was positioned somewhat differently with respect to client and government documents and needs, carrying out a different action, in a distinctive form. Yet together, in comprising a case file, they together defined the actions taken on behalf of a client in a case. Schryer (1994) similarly has examined the way alternative reporting forms for veterinary care are tied to basic alternatively different versions of what the activity of veterinary care is about; 
she also notes how adherence to one or another form covers over unresolved tensions and contradictions in the field.

Certain central documents may take a major role not only in defining the terms of a social activity system, but in organizing the genres of surrounding discourse. McCarthy (1991), for example, has examined how in psychiatry the Diagnostic Statistical Manual in its various editions and revisions was intended precisely to be such a vehicle of disciplinary organization and has succeeded in creating a common nomenclature and nosology (taxonomy of diseases or disorders), and has influenced all other documents of the field from the production of case notes to admission documents, case write-ups, patient records, and insurance reporting forms. A follow-up study (McCarthy \& Gerring, 1994), however, also reveals how political negotiation of nomenclature leaves fundamental medical contradictions unresolved beneath institutional decisions. This is similar to the findings in Bazerman, 1987a that institutional regulation of the forms of reporting in experimental psychology achieved through dominant groups in the American Psychological Association in the middle of the twentieth century, kept unresolved contradictions in the field that became visible again in the latter part of the century as theoretical interests in the field opened.

Related genre work (reviewed in Russell, 1997b and Bazerman, 2008) makes similar points. In order to provide some theoretical model for these organizational coherences, I presented a model of how genres stand in recognizable relation to each other within social groupings, often with implications for typical and coherent sequences of production of documents within social structural constraints (Bazerman 1994a, 1994b). Thus, for example, in classrooms, syllabus sheets assigning readings are typically followed by students reading those assignments in advance of lectures and discussions; these are then followed by paper assignment sheets, submission of papers, and teacher comments. All ends with exams and grade sheets. Any missing or weakly performed component in this sequence disables the continuation of the genre sequence and learning activity, and disorganization of sequence can lead to incoherence in the activity.

Russell (1997a) explicitly ties this notion of systems of genre to Engeström's model of activity systems, with attention to the particular problem of understanding the relationship of classroom activity systems with various public and professional discourses related to the course discipline. My book on the Languages of Edison's Light concretely attempts to trace the historical development of the discourse activity systems Edison must engage in and then locate his interventions within specific moments and sequences of utterances within these activity systems (Bazerman, 1999a). 


\section{REFLECTIVITY IN INDIVIDUAL AND GROUP WRITING ACTIVITY}

Because writing is embedded within social systems, both the activities and the systems are open for reflection at each juncture. Indeed almost every act of writing requires reflection and thought-in part because the writing is likely to occur at some physical and temporal remove from the exigencies that drive it and the people who are to be influenced by it. Only in very immediate and brief writing, such as when we are asked to fill in our name on a form by a clerk standing next to us, might we carry out writing with little thought. Rather, acting through a second order symbolic system with signs on the page to contemplate, we are likely to think about what we are doing even though the depth of contemplation and understanding may vary,

This reflective cognition opens up the opportunity for rethinking our aims and our place within the activity system. Each new act of reading and writing reinvigorates and in a sense remakes the activity system, carrying it forward. The more we are able to reflect on the system, the greater the possibility for adjustment, change, remaking, and reinvigoration. The reflection may be directed at any aspect of the activity system, whether the choice between two near-synonymous words to evoke different sets of associations or the choice of fundamental strategies to engage audiences in issues they have not been attending to. Even attempts to transgress, surprise, or disrupt require reflection on the usual patterns of discursive activities so as to know where one might most effectively plant one's provocations and disruptive surprises.

At the same time as refection allows potentially broad-ranging contemplation, creativity, and reconfiguration of activities, the orderliness of activity systems serves to reduce the necessary sphere of contemplation, perception, and cognition-as suggested by Edwin Hutchins' (1995) study of navigation techniques in traditional cultures and modern naval systems. In the cases Hutchins studies, the techniques and tools of navigations focus the individual navigators carrying out specific limited tasks which are then collected and coordinated by other collaborating individuals. Each person only has limited tasks to accomplish. For a deckhand on a naval aircraft carrier this may mean aligning an identified site point to a cross hair in a sighting tool and then at designated moments calling out a number indicating the placement of the crosshair on a scale on the navigational tool. Such numbers permit the captain to align a caliper-like tool on a map, marking the position of the ship and setting a line for the continuing course of the ship.

For writers, the orderliness of genres constrains and focuses the writing task. A person writing a research report on a psychological experiment knows 
specific things should be attended to and specific kinds of information should be reported in the text according to a fairly stable and recognizable organization, deploying standard formulations, techniques, and phrases. One hardly has to create a text ex nibilo or search the whole world for relevant material and phrases. Similarly, someone reading that report can approach it with a fairly focused set of expectations of what to look for and what interpretive and critical techniques that need to be deployed (Bazerman, 1985, 1987a). Writers and readers can, therefore, limit their conscious reflection and choice-making to a few issues, unless they uncover serious contradictions, problems, or limitations that challenge the standard way of doing things and taken-for-granted knowledge. Such contradictions and disruptions may lead writers and readers into what are considered the deeper questions of the field. In research and knowledge-producing fields these deeper questions concern assumptions, standard investigative and argumentative procedures, the codified knowledge relied on from the literature, theoretical predispositions, and the very social organization of the epistemic or activity field. Each of these questions has consequences for writing. Similarly, in business fields such issues as basic economic relations, marketing and production strategies, organization and task structure, and representation of products all have consequences for reshaping writing and organizational collaborative writing practices to carry out one's business effectively.

\section{MEANING, CONSCIOUSNESS, AND ACTIVITY: LURIA}

The third member of Vygotsky's troika, Alexander Romanovich Luria, focused on functional systems within the individual rather than within the social activity system, as Leont'ev had. Luria became widely known in the West for his work on cognitive neuroscience, which grew out of his work on brain damage and aphasias. But his work was directed by interests that preceded his work on brain physiology, and his findings in neuroscience are consistent with his findings from psychological and developmental studies. He viewed the brain not as the aggregate of specific locales each with discrete knowledge or directing a discrete skill (nor did he take the extreme opposite view that the brain operated only and always as a whole), but rather he viewed the brain as the differentiated neural ground on which functional systems developed as the child grew through activity-driven social experience and learned language which mediated most social activities. The development of spoken language was particularly crucial for the development of higher mental processes and functions. These functional systems brought into dynamic relation multiple parts of the differentiated brain directed from the cortical regions. Spoken language provides the means 
for conscious and voluntary action and is implicated in all higher mental functioning (see Vocate, 1987).

We can see Luria's interest in role of language most explicitly in the twin study (Luria, 1979; Luria \& Yudovitch, 1959). A pair of five-year-old male twins, as a consequence of delayed phonological development, had only limited and idiosyncratic language development, and largely communicated only with each other. They mostly played with each other in embodied action, rather than language-guided interaction. When entering a kindergarten they did not play much with peers and had limited abilities in such creative tasks as blockbuilding or role playing. They had almost no narrative or planning speech. After investigators separated the twins to aid in their social, linguistic, and intellectual development, the one with the least language development was given special instruction in discriminating and articulating sounds and in engaging in adult speech. After ten months of separation both children had developed in their general use of language and in their use of narrative and planning speech. Even more strikingly, the more backward child who received special instruction wound up using more narrative and about the same amount of planning speech as the child who was originally more advanced linguistically. Both the planning speech and narrative speech of the child with extra training were more likely to apply to objects not in the immediate environment. Further, this child had a greater ability to comprehend complex grammatical constructions and inflections. The growth in language of both twins correlated with major changes in their play incorporating objects into their plans and game rules. The play became restructured around verbally formulated projects and articulated objectives. The child with additional training, although previously the follower in the twins' play, had become the leader, learned more rapidly, and assimilated more easily new learning into his activity.

Throughout his career Luria carried out a number of similar studies, from his early studies with Vygotsky on the use of signs to aid in the organization and regulation of tasks to work in the 1950s on verbal regulation and inhibition of behavior. In these latter experiments, for example, two-year-olds, holding a rubber bulb, when told to squeeze the bulb when a red light appeared, would immediately squeeze upon hearing the word "squeeze." Further, they would not squeeze when the red light appeared. Children at age three and four, however, were able to follow instructions, regulating their behavior accordingly. Further complications appeared when children were given two differently colored lights to respond to or were given instructions not to press. Not until age six could the children consistently regulate their behavior according to complex verbal instructions, although even the youngest child subjects could repeat the instructions and apparently understood them at a verbal level. Children 
with learning disabilities had greater specific problems with these tasks through later ages. Luria interpreted his results around the development of higher order regulation of complex behaviors based on the internalized meanings of words gradually overtaking "natural" responses regulated by immediate stimuli (whether material or verbal) (Luria, 1961).

These studies provide some insight into Luria's longitudinal study of someone with an extraordinary memory begun in the 1920s and continuing into the 1950s (Luria, 1968). The subject's powerful eidetic memory worked through direct images, associating words with verbal images, synesthesia, and direct sensory perceptions or associations, but the memory seemed unordered, unregulated and undirected by verbal means. At times the subject even failed to notice the sense, meaning, or logical sequencing of material he memorized. He constructed complex eidetic schemes where a simple noticing of symbols, such as a numerical or alphabetic sequence, would serve. His typical method of ordering was to place various images along a mentally imagined road or a hallway, and then to mentally walk down the road calling out what he saw. These memories were so powerfully planted as direct sense perception, as eidetic memory, that his mind became cluttered with unforgettable images, particularly as he earned his living as an entertainer performing prodigious feats of memory. He tried various devices of sensory imagination to expunge these memories, such as erasing a mental blackboard or covering it over with a canvas. However, such devices frequently failed and memories of images from previous memory performances would return. He then attempted to write the material down which he wished to forget, under the reasoning that if it were written down he would no longer need to remember. But even this did not work. Only when he noticed that a memory did not appear and he was able to tell himself it was because he didn't want it to appear that he was no longer bothered with unwanted memory. Although this process, as Luria notes, is somewhat mysterious, the verbal regulation of his mental process, announcing to himself that he did not want to remember these images, was an important part of the process.

The role of consciousness as substantive parts of people's life is thematic in Luria's work. Investigating the role of consciousness in a person's mental operations and behavior led Luria to what he called a romantic science, which attempts to understand human mind and behavior in all its richness rather than to reduce psychology to abstracted principles. Thus his study of a man with a war-time brain injury (Luria, 1972) was not so much the story of a reduced capacity as of how the person coped with the new conditions of mental life he found himself living with. Within the capacity and tools available to the self, a person must create functional systems to carry out the operations, actions, and activities of life. Under normal circumstances many of the functional systems 
arise in a preconscious coordination of the parts of brain and behaviorthough complex functional systems may develop later in life, building on early established functional systems and integrating tools, artifacts, and social organization. Under more extraordinary conditions, the individual must consciously create new functional systems to do what other people do without conscious thought, such as relearning to read after parts of the visual processing mechanisms having been destroyed by brain injury. In recent decades Oliver Sacks has pursued romantic science exploring individual personalities coming to live with atypical neuropsychological or perceptual conditions (See O. Sacks, 1985, 1989, 1995, 1996).

One of the important implications of functional systems and their reconstruction incorporating tools, artifacts, and social organization is that the specific cognitive functional systems involved in reading and writing need not have evolved over a long biological heritage nor be present and activated in the early development of embryo and infant. Rather, they may develop late within human history in concert with the historical emergence and elaboration of the potentials of written language over the last five thousand years, although built on upon earlier and longer-standing human biological capacities and social inventions, such as language. The functional systems associated with language become transformed and reconfigured as they are applied to written language. Thus, while directives and even principles of justice could be articulated in purely oral conditions, only with the emergence of written laws could the relationship of large and complex sets of laws be readily examined, compared, regulated, and ordered. The cognitive functional systems of modern legal thought would not only be of little use under oral conditions, they would have little occasion to be used and therefore to develop.

Similarly, in each child organized approaches to reading and writing emerge only well into a child's development, typically at the fourth or fifth year or later, building on earlier biological capacities, cultural resources, and social experiences. Even at the level of visual perception, eyes need to be trained to focus on small marks on a page (which the young child notices older children and adults orienting towards), scan in the organized path of the particular writing system (such as right to left and then down the page), make fine discriminations between letters, organize letter perceptions within words, and then regulate it all by assigning meaning to the collections of marks. Similarly, learning to inscribe letters requires the development of functional systems that are dependent on cultural practices embodied in the writing system (such as alphabetic, syllabic, or ideographic), the technologies of inscription (stylus, pencil or keyboard), and the associated motor skills. Beyond these most basic skills are the many systems of interpretation, contemplation, personal association, evaluation, 
stance taking, synthesis, and idea building which may occur largely internally (though having their origin in some interpersonal experience and training). The development of our cognitive capacities employing literacy is a major theme of the entire educational system.

Since literacy is such a late arrival in human evolution, it is unlikely there are biologically determined pathways for the cognitive development of the fully functional systems of literate participation, although some lowerlevel components such as visual discrimination and motor control do have biological substrates. Consequently there is no biological guarantee that literate systems will regularly develop in different individuals in the same way. That is, individuals may address the challenges and solutions of meaning making from signs differently. Each person building on biological constraints and affordances, must innovatively build their own functional cognitive systems out of their experiences, instructions, and the existing prior relevant systems they can bring to bear to the task.

Because literacy involves such a later-adopted restructuring of consciousness around newly developed functional systems that embody and adapt to new cultural tools, Luria clearly distinguished between spoken and written language and had only limited comments on writing, primarily to distinguish it from spoken language and to identify its onset as a new stage in development. In an early article, from 1929 (Luria, 1978), he points out that children first learn writing only as a series of scrawls, thinking that this external practice is the full extent of what is entailed in writing, and only later does the child start to develop an understanding of how signs are distinguished and meaning mediated by them. Thus the process of understanding meaning transmission and construction within literacy does not flow directly and naturally from an understanding and use of spoken language, but develops through the formation of new functional systems. Writing near the end of his short life Vygotsky (1978) has similar but more developed comments about the way in which children move from a sense of writing as an external practice to a sense of graphic symbolic communication through drawing and then only after the transcription of sounds that themselves convey meaning - a sign of a sign, as Vygotsky says. That is, in alphabetic languages the letters signify sounds and then the sounds are the vehicles of the meaning.

Following this perception that different functional systems must be developed to process meanings embodied in written language, Luria says that higher mental processes have two distinctive components that differ in origin, function, and structure. Among the differences that Luria notes between learning speech and writing is that the embodied physical context that usually accompanies spoken language aids in its interpretation, whereas written language must typically 
carry more of its situation and meaning through its own verbal presentationthus adding a conceptual abstraction of situation to the abstraction of phonetic expression and the relation of phonetic expression to meaning.

Because of this removal from the immediate situation and the engagement with texts that seem to draw us out of our immediate behavioral contexts, written language is much more deeply implicated with inner speech than spoken language. While reading and writing may originally have been associated with spoken performance and rehearsal of texts-scripting of speeches and communication of personal messages through letters read aloud-yet over time written language use moved inward as people read extended texts to themselves whether or not they vocalized the words or adopted the later practice of silent reading. Similarly, writers as they gain in skill develop ever more extended texts, prepared at their isolated desks to be delivered for other people's contemplation. The semi-privacy and delayed release of writing has created extended space for composing processes of interaction with one's own emerging text, available for planning, reflection, evaluation, self-censorship, revision and refinement. These composing activities support the development of more elaborated and extended consciousnesses. Luria noted this very close association between written language and inner speech, and suggested this as another reason writing needed to be considered separate from spoken language in its effects on cognition and consciousness (Luria, 1970).

In order to explore this new level of consciousness that Luria and Vygotsky associated with the onset of literacy, they undertook some expeditions in the 1930s to Uzbekistan and Kyrgyzstan in central Asia in order to understand the reasoning processes of peasants with little experience of schooling or literacy. Using ethnography, interviews, and puzzle tasks, they found that those with less schooling tended to answer questions and solve puzzles more on the basis of their own experience and immediate knowledge than on logical abstractions, deductive reasoning, superordinate categorization, and similar devices associated with uses of literacy in schooling. While they attributed the differences primarily to the acquisition of literacy, there was no attempt to disentangle the effects of cultural experience of schooling from the learning of literacy, nor was there any attempt to document the particular experiences and uses of literacy within the lives of the communities and individual studies. Rather literacy was treated as an undifferentiated new stage of consciousness. The studies of Scribner and Cole (1981) disentangle these effects more precisely, and point toward how culturally specific the uses and practices of literacy are and correspondingly how specific and varying the cognitive consequences are.

Scribner and Cole's studies were in response to a large number of studies during the sixties and seventies that explored the cognitive consequences of 
literacy (by such people as Goody, 1977; Havelock, 1971, 1981; Ong, 1958, 1982) that considered the consequences of literacy to be general and uniform. When this earlier work is reinterpreted through lenses of cultural specificity and social history (as Goody began to do in the Logic of Writing and the Organization of Society, 1986), this opens up an analysis of how human cognition has changed in relation to the emerging functional social systems of literacy (Bazerman, 2006) as well as the cognitive functional systems of individuals (Bazerman, 2009).

Since literacy itself is an historical cultural accomplishment, we would expect cultural practices to loom large in guiding individual development, such as the Jewish practice of placing honey on letters so the child can associate written words with basic biological pleasures. The frequent ritual oral repetition of certain communal texts in public can shape the functional systems of literacy as can extensive, structured phonics instruction, or the ambient profusion of texts incorporated in daily life activities. Our ways of incorporating literacy into our cognitive practices can be influenced by a cultural expectation that we use literacy to memorize and hold texts precisely fixed or that we use it for creative projection of personal meanings. Equally, social environments of argument over texts, or of fear of the power of words to control one's life, or of irreverent humor supporting heterodox culture will all influence how the individual orients toward literate activity and constructs functional systems to participate.

Our functional systems of literacy develop in relation to social circumstances and practices and in relation to our capacities evoked in such circumstances. Even within homogenous cultures, individuals may come to interpret texts differently and to write different texts, both within the bounds of orthodoxy and on the transgressive edge of heterodoxy. When cultures support profusion of experiences and novelty of expression, the individuality of development flowers into great differences of interpretation and expression in many domains, from poetry to business plans to theories of fundamental particles. The styles, relations to audiences, text organizations and processes proliferate as individuality of literate experiences is supported and rewarded. Writing development, rather than moving towards a single ideal, proliferates differences and the most developed writers write the most uniquely, even though some limited aspects (such as spelling, grammar, or even preferred style) may be regulated by cultural norms. The importance of both culture and individual experience in writing development bring together Leont'ev's social approach to functional activity systems and Luria's more individual approach to functional systems. Individuals develop their internal functional systems of reading and writing while participating and establishing roles within the communal 
functional activity systems in evolving societies. This interconnection between individual and social development should caution us against over-generalizing about the cognitive systems engaged in literacy, even though reading and writing are fundamentally cognitive acts of meaning making.

The elaboration of Vygotsky's work by his collaborators and heirs, Leont'ev and Luria, helps give further shape to our understanding of humans as active social symbolic selves, developing consciousness in relation to language uses that arise within our organized social lives and employing our historically developed cultural tools. Spoken language and then written language transform consciousness and allow us to participate in more complex and reflective activities and actions. In the next two chapters we will explore some parallel developments in European and American social science that provide different perspectives on the forms of expression, consciousness, and social organization that have been intertwined with the development of literacy. 



\section{ACTIVE SOCIAL SYMBOLIC SELVES: THE PHENOMENOLOGICAL SOCIOLOGY TRADITION}

By reading and writing, people act socially and symbolically, constituting themselves, their orientations, attention, relevancies, and consciousnesses in relation to social communicative interactions. The Soviet Russian psychological tradition, as we have seen in the previous two chapters, provides some means for understanding how the individual within social interaction develops the means for self-regulation of behavior and for carrying out social interaction, forming a cognitive organization and a consciousness built upon the neurobiology of the brain that becomes elaborated through participation in social activity systems. In the course of developing such social selves individuals are also building a social world, a world saturated with meaning and human activity. Reading and writing are means not only of building individual consciousness and shaping individual action of the literate person, they are also means of the developing the collective thoughts and interactive organizations of the societies within which individuals develop their lives and consciousnesses. Thus there is a dialectical relationship between the psychological and sociological.

For example, the texts of popular political parody, government constitution, political theory and analysis, and the like provide the terms within which politically oriented youth develop their personal thoughts and in which politically engaged groups develop their ideas and plans. They are also the means by which individuals and groups engage in political action, attempting to influence candidates and issues. Politically oriented youths affiliate with groups, extend their awareness of the texts viewed relevant, and seek to make their ideas more widely known and directly realized in the workings of government. In doing all this they contribute to a climate within which further new generations will form their political consciousnesses and engagements. Of course, immediate face-to-face interaction, experience, need, and passion are important shapers and drivers of political culture. Nonetheless, in a literate world through texts individuals learn facts about situations occurring outside their immediate observation and gain access to ideas and experiences of nonpresent others. Texts, additionally, can be used to plan and coordinate work of local and more extended groups. Further, modes of thought and analysis 
characteristic of literacy are likely to influence political stances and shared texts are likely to be discussed and form common bonds among the politically active. Even more, the reach and delivery of political words and news of actions are extended and transformed through the circulation of texts, so that individuals and groups orient to larger political units beyond the local town such as the province, nation, or international bodies. Thus the nature of political individuals and political culture change through the communicative means which form the medium of knowledge, thought, expression and action for both individual and group.

The next four chapters explore some theoretic grounds for understanding the relation of psychology and sociology by examining several related traditions of European and American sociology arising out of phenomenology and pragmatism. Along the way we will draw some connections with Russian sociocultural psychology. The aim of examining these several traditions remains the understanding of the modes of being that are developed, carried out and transformed through the inventive means of written language. While this account may at times appear to extend some distance beyond writing, we will regularly return to consider implications for literate modes of interaction and being.

\section{SCHUTZ, THE PROBLEMS OF ECONOMIC BEHAVIOR, AND A UNIFIED SOCIAL SCIENCE}

Miller's (1984) move to see genre as an instance of Schutz's typification process (Schutz 1967b; Schutz \& Luckmann, 1973) provided a key link in our understanding of text as social action and as constitutive of the social order. Miller's recognition was paralleled independently by Schutz's followers who considered genre as a typifying force. Here we will be looking into the problems and reasoning that led Schutz to his central concept of typification and to some of the extensions and implications he drew. This examination of Schutz will provide resources for understanding genre as typification and its role in constituting individual consciousness and social order. This examination will also provide a vehicle for understanding the relation of contemporary genre studies to the several lines of sociological research that have been deeply influenced by Schutz.

Alfred Schutz (1899-1959) was a banker, economist, and social philosopher, first in Vienna and then migrating to New York in 1938 when Germany annexed Austria. As a member of the Austrian school of economics he was much concerned with grounding issues of economic behavior and, by extension, grounding issues of the social sciences. After immigrating to 
the United States he continued his career as a banker but also affiliated with the New School, becoming the foremost spokesman for phenomenology and phenomenological sociology in the post war period, influencing many developments in microsociology, ethnomethodology, conversational analysis, and interactional analysis (Heritage, 1984), to be examined in Chapter 7.

While most commentators see Schutz's career in banking and economics as separate from his philosophic interests, Prendergast (1986) persuasively identifies the roots of Schutz's interest in processes in social typification lying within problems of economic behavior that troubled him and his colleagues in Austria at the start of his career. The problem may be stated as follows: The principle of marginal utility (the most distinctive contribution of Austrian economics) rests on a simplified model of human behavior as dictated by a rational calculation of self-interest, based on knowledge of markets in relevant goods with few extra-economic, extra-market considerations. This model is the well-known homo economicus acting with other rational self-interested individuals in a market which contains all information necessary for acting within it. The concept of homo economicus stands behind much of modern economics and dates back at least to the time of Adam Smith: it has also been from the beginning regularly critiqued as a narrow fiction. Schutz wondered how it was that this clearly fictive assumption that reduces the complexities of human behavior in patently unrealistic ways still produces accounts of behavior that are highly predictive for economic behavior within markets. Further, he wondered, given that we have no direct and unmediated access to the thoughts of others, how can we with any confidence make any assumption about their motives and the meaning of their choice making.

In grappling with these problems, Schutz turned to Husserl's phenomenology (1964). In so doing Schutz transformed phenomenology from a philosophic inquiry into a sociological method and ontology, as a way to understand how individuals came to act and attribute meaning according to socially constructed ideas and structures. One of the major vehicles for this transformation was a synthesis with Max Weber's sociological method of ideal types, which Weber saw as the fundamental method of sociology. Schutz (1967a) argued that ideal types were not only an analytical method of sociologists but also the practical method by which individuals made sense of their social world, developed guidelines for their own choices and behavior, and came to attribute meaning to their own actions and the actions of others. In proposing a solution to the problem of economic behavior using a general philosophic method of phenomenology and a general sociological method of ideal types, Schutz developed an approach to understanding all forms of social behavior and a general model for a unified social science. 


\section{SCHUTZ'S TYPIFICATION}

His proposal, in short, was that individuals, in order to participate in what they see as a meaningful or useful social arena, take on what they believe to be principles of that arena. They then use those principles to guide their own behavior and to make their behavior meaningful and intelligible to other participants. Whatever their underlying motives and thoughts are, as the impulses become realized within social action, impulses take on the forms of social types. Those types in turn provide a cognitive orientation for the individual, establishing patterns and principles of thought and identifying relevant knowledge that the individual brings to bear on the circumstances. Thus if you depend on a market where you trade goods and money based on their perceived value to you, you will begin to adopt a perspective of marginal utility, calculating which goods would grant you the greatest satisfaction of desires, given the relative prices of goods, the amount you already own, and the additional cost and pleasure attached to each increment under current market conditions. Further, you will gather information relating to goods, desires, the desires of others with whom you would buy and sell, and so on. In short, you would develop the consciousness of a rational economic actor in the market in the course of making choices within that market. The principles of economic behavior are therefore not essential and unchangeable facts of human psychology-they are rather patterns of behavior associated with particular market formations which individuals orient towards and adapt themselves to by acting typically within such markets. Even if individuals are not at first oriented towards markets but find themselves living within market economies, where to meet their needs and desires they by necessity must adopt a market orientation, they are drawn into a nexus of economic reasoning which may come to dominate their life-world. Thus laborers as much as capitalists are drawn into the cash nexus. In a cash economy, monks are freed to contemplate other matters only if they have a protective institution that takes care of economic matters for them.

Although Schutz seemed unaware of it, Adam Smith, a century and half before, advocated the idea of rational economic behavior as a social fiction that if adopted by all people would provide a basis for an economic socio-political order. Thus Smith attempted to enlist people into the very capitalist system of economic behavior that Schutz was grappling with. Smith hardly believed that humans naturally operated as homo economicus; rather, he followed Locke and Hume in seeing individuals having idiosyncratic experiences leading them to developing idiosyncratic sets of associations, with consequent divergent perceptions, desires, beliefs, behaviors, and guiding principles. Smith did, however, note that the general patterns of life and economic circumstances- 
the activities and forms of production one engaged in as well as the particular circumstances and dilemmas of individual lives-provided some clues on which to construct a sympathetic understanding of the range of thought and knowledge as well as the particular choices they might make. He further noted that humans had the unfortunate tendency to affiliate with and be deferential to those whom they perceived to "be their betters," those who stood hierarchically above them in contemporary systems of social order. People, nonetheless, also had a tendency to want to better themselves within those same circumstances and orders. Humans in Smith's eyes were hardly rational creatures. They tended to remain committed to hierarchical societies dominated by church and monarchy, despite the exploitation and lack of needs satisfaction they experienced within those social orders. He did feel, however, that they would be more likely to pursue their own interests more consistently and rationally (and therefore participate in a society more directed by local knowledge and providing for a greater satisfaction of their perceived needs), if they were convinced to pursue their impulse to better themselves and if there were available a universal mechanism of exchange that would allow them to pursue their own individual notions of betterment (Smith, 1978).

Money and markets were to be that mechanism. If people could be convinced to see money as the universal means to satisfy their diverse needs, desires, and interests, then they would all pursue those interests through economic exchange within markets, which would then provide a universal site of social ordering and affiliation. As Marx (1909) would say, all would be drawn into the cash nexus once they adopted this economic attitude, and this nexus would be selfregulating and universally motivating, and thereby be so powerful as to provide a compelling alternative to hierarchical forms of social domination.

Smith's project to enlist people into market behavior directly implied the Schutzian concept of typifications. The types of behavior appropriate to markets would frame modes of consciousness, into which desire and motive would be channeled. Schutz, however, generalized this idea beyond the economic rationality that one engages in to participate in markets to the forms of consciousness one develops in participating in any specialized domain. Thus a chess player in entering into a chess game adopts certain motives and principles of choice-making that comprise the typical attitude of the chess player. As a person becomes more serious in their orientation and psychic commitment to the game, that person takes on the typical motives and consciousness of a chess player. We can readily specify the kinds of things the player wonders about, the kinds of plans they make, and the kinds of choices they confront. As the person moves through various levels of skill, we can even begin to specify some of the different levels of consideration they are likely to process rapidly or even know 
automatically. We can as well identify some of the new areas of consideration where they are likely to attend to more consciously and intently as they advance in their skill. Indeed learning at the higher levels in part means learning to attend to the kinds of considerations the better players attend to. These levels are historically emergent as newly discovered principles of useful strategy; thus the history of chess thought is usually recounted by the types of strategic and tactical thinking introduced in each period. Further, as chess becomes a larger part of a player's life, such as among professionals, we can specify the kinds of concerns and orientations they adopt in building a chess career and adopting the chess way of life.

Equally we can say that the fourteenth century Venetian entering into the world of courtiers, or the late nineteenth century educated New Englander entering into the world of poetry, or the twenty-first century web designer entering the competitive workplace each absorb the modes of thought and action they perceive as typical of those domains, even as they attempt to innovate in their interests and establish their distinctive qualities within the criteria of excellence of their times. This is the insight developed by Bourdieu in analyzing the fields of artistic production and the ways one distinguishes oneself to adopt a unique position within a structured field of endeavor (Bourdieu, 1984, 1993). Insofar as the person is immersed within a specific domain and activity system, we can begin to describe the orientation, motives, organization, and typical contents of her or his consciousness, and describe her or his individuality in dialectic with the typicality of the time, place, and domain.

Further, our familiarity with that domain and the cognitive orientation of the other participants allows us to understand and appreciate what others do. People in an economic market can make deals with each other because they know reliably what kinds of actions the other will take along with their motives and reasonings. Thus they can shake hands on a deal, knowing paperwork, products, and payments will follow in what is considered a timely and appropriate way. Of course, they are also aware of the kinds of ways others may attempt to take advantage of them and are on the lookout for the forms of cheating that have developed in those domains. At the same time, experienced practitioners will be in a position to recognize what might count as a reliable partner and a good deal. They can also appreciate the truly novel or clever move in their world in the way outsiders cannot. Similarly, skilled chess players are able to understand the moves of players at or just above their level, and even appreciate a move that pushes the play to a new level or escapes the bounds of the expected to gain an unanticipated advantage. The meaning or value of that admirable move would escape players at a level that has not yet introduced them to the way if thinking that makes the move intelligible. Courtiers can understand what other 
courtiers are doing, as can people initiated into particular poetic worlds best understand what poets in that world are doing, and experienced web designers can understand the meaning and motive of an innovation.

The many practical guides to participating in a field (whether how to invest in the stock market, be a courtier, play the middle game in chess, or design web sites) provide information for people entering into a domain, before they have internalized these typical orientations and guidelines into their own practice. Such guidebooks attest to the strong impulse we have to reflexively understand and articulate the principles of acting within particular spheres and the need for training in specialized modes of thought to maximize performance. Similarly, the many interpretations and appreciations - whether accounts of exemplary courtiers, analyses of chess games, interpretations of poems, or critiques of web-designs - suggest how specialized knowledge of the typified act aids understanding and appreciation, thereby expanding our consciousness within the realm, giving us more resources to think with when engaged in that realm.

\section{THE TYPIFIED INTERNAL AND EXTERNAL, AND THE FALLING AWAY OF THE UNTYPIFIABLE}

This understanding of typifications never lets us into the full stream of consciousness of the individual, as Schutz points out. Rather, it only lets us anticipate the general outlines of consciousness, and then read back from the particulars of public performance, behavior, or accomplishments what the individual was likely to have contemplated and intended specifically. The thoughts that the person rejected as irrelevant or the thinking about other things (worrying about Uncle Joe's health while playing the chess game) that do not obviously derive from the activity or resources translated from outside the domain are opaque and invisible. In the emergent production earlier thought, contemplated behavior, and mood fall away, and we are only left with what the person brings into the public arena in the form they bring it. The individual acting too is left with the emergent public product as a public commitment and identity. Ultimately all that mediates between us is what has been externalized (See Thomason, 1982).

Our prospective motives of what we might desire to do, furthermore, are directed by our understanding of the way the action realm works and what actions are typically successful. We can only anticipate going to market to sell our agricultural produce if a marketplace exists, and we can only desire to learn the musical instruments we have seen and heard. Even if we are to innovate in organizing a public square to transform prior irregular trades or to create 
a new stringed instrument, we still work from what we have seen and heard before to imagine improved possibilities. Just as we form our own participation and orientation out of what we internalize from the activity world around us, what we add to that world is only what we manage to externalize. It is those externalizations that provide information for others to understand our behavior within its typified realms, and for them to construct their constantly evolving and emergent notions of what is typical in those realms, thus orienting their own behaviors and consciousness. They now can organize their lives to come to the regular Tuesday market, or they can learn to play a new instrument and explore its musical potential. Further, our own reflexive awareness of our actions within that typified realm provide us information about our own identities, commitments, and actions, upon which we may base our after-thefact accounts of motives. We become merchants or mayors, violin players or composers, as we participate and succeed in our activities and carry on future actions based on our experiences and successes.

This dynamic dialectic of internalization of existing social forms to provide the grounds of action and the externalization of material actions with material consequences that remain after the evanescence of our internal processes creates some of the deepest puzzles and tensions of writing. This dialectic is in the difficulty we have in locating or composing the state of mind from which our meanings will flow; it is in the feeling that our words do not fully reflect what we feel; it is in the surprise we find to read what we have written; and it is in the surprise we have when we find others understand something different in our words than we intended. This dialectic is also in the contradiction between the conviction we may have that the meanings we get from texts seem so profound and robust and yet the recognition that written language is a fragile vessel for evanescent cargos of internally perceived meanings. (For an elaboration of internalization and externalization processes from a Vygotskian perspective, with specific attention to the role of concepts and concept language in writing, see Bazerman, 2012).

\section{RELEVANCE IN CONSCIOUSNESS AND EXTERNALIZED MEDIATIONS}

The specific resources, knowledges, memories, and other contents we bring to bear in constructing these externalizations and that we represent within these externalizations are driven by our sense of relevance for the project at hand. We assemble what we think we need, based on what we think we are doing, shaped by the typified project and the typified rules that we adopt as part of engaging in 
and understanding the project. Of all those things that might potentially come to our mind or that we might search out in the library and world, our attention focuses on just those things we view as enabling us to do what we are doing, as we understand what we are doing (see Bazerman, 1985 for an example of how writing intentions influence reading choices), and it is just those things that are then available as we assemble our actions. For the process of writing this means what we bring to bear as we assemble the text and what we display in the text arises from a project-shaped consciousness. We are thinking to write and we are writing with the contents we have mentally assembled as relevant within the typified understandings of our projects.

A second kind of motive for identifying relevance occurs after the fact when we need to give an account of our actions and the conditions that gave rise to it, to justify or explain or take lessons from our actions. What is considered relevant in such accounts, both in what enters our consciousness and emerges within our externalized accounts, is what we understand to be relevant to such accounts and which we believe our audiences will accept as relevant-and thus we are accountable in typified ways. In our original projects, in fact, we may anticipate such accountability needs by acting so as to provide evidence of the "appropriateness" or "reasonableness" of our actions. Some projects within their typifications already establish the need for such accounts of their coming into being, as a scientific experimental paper requires an account of the theory, previous findings, experimental methods, and laboratory events that are claimed to have brought the experimental investigation and consequent paper into being. That these are cleaned up to make more coherent and acceptable accounts in the final externalized version (see Medawar, 1964; and Bazerman, 1988 ) is precisely to be anticipated given the Schutzian observation about the emergent shape of typified behavior and consciousness, and the slipping away of those things viewed as irrelevant, outside the project, or non-normative within the activity world. Typifications in this way control our horizon of attention and what is viewed as unproblematically appropriate to such actions, and accordingly obscure those things that might be perceived as not worth thinking about or being discussed as part of the project we are engaged in.

\section{THE NATURAL ATTITUDE AND THE PULL OF TYPIFIED CONSCIOUSNESS}

Typification and relevance are so strong in shaping our consciousness and horizons of attention as we are drawn into realms of activity and relationship, that it is hard to remember that we could be thinking, perceiving, or doing 
in any other way. Berger and Luckmann (1966) elaborate the reconstructive nature of autobiography and memory to make sense of our lives. Further, we tend to see things from the normalized perspective of their after-thefact accomplishment rather than their in-the-making assemblage. Latour (1987) in considering the difference between accounts of science-in-themaking and science-already-made shows how much is obscured by viewing completed projects as accomplishments rather than still open puzzles. What we patch together through contingent choices thus comes to appear as the full recognizable, natural, and complete acts rather than shaped by an historical process of social construction of typifications.

This inability to see beyond the habituated typified order has been called the natural attitude by Husserl (1964). This is particularly true of socially pervasive practices which we are drawn into from earliest childhood, such as systems of morality or family relationship or street navigation or communication through language. Only through some unusual experience, reflective position, or intentional inquiry are people able to step out of their naturalized world to begin to perceive its arbitrariness, to see that there can be fundamentally different ways of going about things, and to recognize those other ways are not $a$ priori inferior or unnatural. When a person starts to learn another language and then finds in it different potentials of meaning, for example, then that person can start to see the limits and particularity of the first language. Similarly for writing there are many understandings and expectations of writing so deeply tied to our primary places of learning of written language and reproduced in institutions and practices of literacy throughout our society, it is hard to see them as anything but natural, the only and right way to proceed with writing and reading - whether at the level of spelling uniformity and adherence to prescriptive grammars or at the level of what constitutes proper topics and selfrepresentation. The practices, situations, and evaluative criteria of schooling have been especially influential in creating our naturalized view of writing.

It is not only the early and pervasively engrained that can be the basis of the natural attitude. When we spend a long time engaged in any practice it is easy to forget that things could be otherwise. Even if in some moods we know that alternative practices, projects, and relevancies are possible, an impassioned commitment to a community or project may foster intolerance of alternative domains of meaning that can be evoked by other approaches to writing. Many scientists, lawyers, or even poets, so clearly engaged in historically emerged literate practices which they themselves only learn in adolescence or later, believe there is only one right and natural way to pursue their projects. Their views of writing correspond to what they believe they ought to be doing as competent practitioners, despite doubts that they cannot in every or any instance live up 
to the normative typified expectation. Self-castigation against an extreme and inflexible typification of competence and the way things ought to be is rampant in many domains of writing. Developing a comparative or historical interest, or engaging in wide and varied practical experiences writing in multiple domains, however, provides a way out of the "naturalness" of current modes of practice to understand the arbitrariness and historical choice making that make expected practices something other than eternal moral truths.

This process of taking culturally developed principles and typifications as commitments for actions and thereby making them concrete in their consequences is the process of reification (Thomason, 1982) - making the ideal or ideological or conceptual materially consequential and factual within life. Reifications of social practices become social facts. This social construction of reified, naturalized orders, however, need not be taken as creating delusions, although in some cases social facts may obscure facts readily apparent to those adopting other perspectives. Reification only means our orienting to, taking part in, and therefore bringing further into being some regime of activity, relations, consciousness, and meaning associated with the invoked world.

We need not be blind to what is happening and how we construct the world we live in. I can be quite well aware that I am entering into a world of chessplaying or music making or legal argument, can be aware of the principles, beliefs, and commitments I take on, and can notice the shifting weights of relevance associated with this world. Equally I can notice the changes that happen to my experience and thoughts as I become more heavily involved in those domains. Indeed, people often reflectively notice and comment on just those changes in themselves and their experiences at moments of transition. On the other hand, we may enter into many regimes of reification long before we have the reflective tools to notice, that we forget our prior states and moments of transition as we move into compelling and encompassing regimes. Or we may lack the motive, opportunity, or position to reflect upon our position. Under such conditions our worldview may become so dominated by the artifice of the regime and is so supported by the perceptions of co-participants, that we become blind to the fact that our investment in this world was elective or accidental. Rather, we attribute those investments and meanings as something natural and eternal, grounded in a moral order that is beyond the human. Violations of the expectations of such unreflective reified practices can be seen as moral outrages and those who commit them as uncivilized, uneducated, or otherwise seriously faulted and needing correction. A reflexive awareness of the reification and naturalization processes that have established school practices and social beliefs about writing can relieve us much of the sense of rectitude and moral outrage that surrounds our view of our own and others' writing. 


\section{CRITIQUES OF SOCIAL CONSTRUCTION AND WAYS OUT}

Nonetheless, the whole idea of social construction has been met by some with a kind of moral outrage, that it is akin to anarchy and nihilism, casting us into radical relativism, throwing all meanings into skeptical disbelief (Sokal \& Bricmont, 1999). Even worse, recognition that all statements of knowledge are socially constructed raises the fear that the material world is unknowable, or rather that adherents of social construction are enemies of the scientific and philosophic projects that attempt to know the world outside of our constructed meanings. There have of course been many books and articles written on this epistemological debate, both throughout the history of philosophy (starting with Plato's quarrel with the Sophists in the Gorgias) and more recently in what have been called the science wars. Without engaging this full debate and sidetracking the concerns of this volume, I just point out that Schutz was very careful to make his concept of reification only a methodological principle, an extension of the phenomenological epoché-a bracketing to hold in suspension those things we take as natural so as to investigate how we take them to be natural. He remained avowedly agnostic on the actually knowability of social and material reality (Schutz, 1967a). The pragmatist tradition, the topic of the next chapter, provides another way of conceiving this issue that get us outside of dichotomies between socially constructed language and the experienced world outside the world of representations. Pragmatism recognizes that we use language as part of our living in material and social worlds with which we have extended experience and in which we have continuing interests. This issue of how we represent our experience of the world is for writing more than a philosophic worry about the status of knowledge; it is a practical problem, as much writing aims at some representation of the world around us. More particularly, writing often draws its force and authority from its claimed accuracy or truthfulness of representation of the world about us. Much writing, moreover, is specifically driven by the attempt to create useful or accurate or truthful accounts of the world we live in and experience.

Finally, it is sometimes claimed that reification (as further obscured by naturalization) necessarily puts a wedge between the created meanings we commit ourselves to and our true natures, creating a false consciousness and giving rise to alienation. However, the view of reification here provides pathways for the realization and development of ourselves; through participating in socially typified projects, adopting the associated reifications, we realize social and cultural possibilities. Reification threatens alienation only if we are drawn into or compelled into typified actions that are not the realization of our own impulses, but the impulses of others at odds with or inattentive to our 
needs and desires. The problem is not in the making of social meaning and the participation in the socially constructed meaningful activities; it is in the relationship between that activity and our own impulses and development, or the organic evolution of social groupings we are part of to respond to changing situations, needs, and possibilities.

\section{TYPIFICATION, NOVELTY, AND PARTICULARIZED MEANINGS}

Being able to articulate our own position and interests within available genres and the associated activity systems can make those genres and associated activity systems continuing sites for our own articulation, development and expression of motives, thereby decreasing alienation from the ensuing discourse. In articulating our interests we bring the particularity of our selves, situations, knowledges, and resources to bear, which introduces novelty in the genre. Participation in many discursive regimes may even require some degree of novelty; it is one of the expectations of newspapers that they make us aware of recent previously unreported events and of scientific papers that they propose fresh findings or ideas to advance communal knowledge. Certain discursive orders may cause us to bring in other resources, other thoughts into the activity in ways appropriate to and intelligible within the type of the activity system and mode of consciousness associated with it. Thus the novelist may draw on personal experiences or historical accounts or new literary theories to make the new novel fresh and different, while still being intelligible and marketable as a novel. However, in becoming part of the world of the novel the original material takes on rules, meanings, and functions appropriate to the world of the novel.

Yet no matter what combination of regimes is drawn on, no matter how individual and subtle these are, they are nonetheless dependent on our mechanisms for meaning making and interpretation in concrete circumstances. This kind of complexity of multiple systems and specific contents is what Geertz considered in his thick description (Geertz, 1980). The building of complexity and novelty of meaning from the fundamental mechanisms of situated understanding is also most relevant in understanding the particularity of individual written statements. People are constantly doing new things through writing, and readers are, with varying levels of motivation and success grappling with new meanings, while still drawing on typifications. Schutz, however, in a number of his most prominent publications has only a single vaguely described mechanism for moving beyond the most gross and distant typifications: getting to know an individual more personally and intimately. $\mathrm{He}$ 
characterizes personal knowledge as something entirely different from typified knowledge, eventually displacing typifications in cases of personal relationship. He sees our relationships on a spectrum ranging from the most typified and anonymous to the most individual and personal, with the great majority of our relationships in the world as being highly typified and anonymous. We know the postal clerk as a postal clerk and relate to that person as a postal clerk. In our office we adopt the role of our professional position and relate to others through those roles. As we develop more personal relationships with others and move out of the realm of the anonymous, we treat them and understand them in less typified ways.

While there is a general descriptive truth to this, I find it unfortunate in implying that in getting to know people, situations, and utterances in greater detail, we put aside our systems of meaning making, rather than invoking them more complexly and with higher degrees of locally relevant information. As a teacher, for example, my knowledge of most students does remain typified in terms of the teacher-student role within educational activities, though when I go from one university to another, I need to develop new models of what kinds of students each campus has, what moves and motivates them, what projects they are engaged in, what backgrounds and skills they have, what local sub-cultures they divide into and are part of. Further I need to learn more about the culture of the classrooms in each place, what students expect to do and experience in different types of a class, how they attend to different activities, and how they evaluate and relate to various kinds of instructors, instructor personalities, instructor statements, and instructor interventions. So getting to know what it means to be a good teacher on a campus means developing through experience and observation a more finely tuned set of typifications which helps me to relate better and more closely with students even if I do not know the particulars of any one of their lives.

In fact, I do gradually learn a certain number of particulars about all of the students, and a great number of particulars about some as our studentteacher relationship develops. We become familiar in the ways appropriate to students and teachers, filtered through our understanding of the expanded types of mentoring relationships. I also learn particulars of their lives that extend beyond the classroom-family difficulties that may interfere with their school work, experiences that motivate them, ambitions shaped over many years, the multiple factors that influence their career choices, the underlying interests that motivate a particular research project. Similarly students learn particulars of my life and interests as I make reference in instruction and more informal circumstances. They may learn about ideas I have had, things I have written, trips I have taken, career choices and struggles-these all may come out in 
direct interchange in classroom examples, advising, or mentoring situations. Or students may find out more on their own as part of personal curiosity about what kinds of persons professors are or about this one individual that is taking a role in their lives. This familiarity framed and motivated by the typifications of teacher-student relationship-a set of typifications that I think it ethical to keep strictly in force and not to confuse with other forms of relationship that would be tainted by the powers and motives inherent in and generated by the teacher-student relationship. All the personalized elaborations of it employ sense-making mechanisms built on typifications of an increasingly refined sort. How do I make sense of a student's motivations in light of their autobiography? How do they make sense of being a student as they recount their lives to me? How do we orient toward each other's comments so as to provide direction for continuing dialogue that carries each of us further down the path of growth and learning that gives meaning to educational relationships?

\section{TYPIFICATION AND PARTICULARITY: APPRECIATING THE MUSIC OF LIFE}

In his essay on "Making Music Together: A Study in Social Relationships," however, Schutz (1951) provides us a more profound insight into how we become attuned to the most novel and subtle sharing of the contents of consciousness with each other. The processes of deep and particular understanding he describes depends on much socially shared typified knowledge at the same time as allowing particular communion over an intricate and moving object that inhabits our mutual consciousnesses to provoke similar attentions, meanings, and motives. Although Schutz draws his examples from nineteenth century classical music culture, his arguments are easily extended to musical traditions even without transmission of written scores, and then to many other forms of collaboration.

Schutz points out that a skilled pianist trained in the European tradition, even when playing an unfamiliar nineteenth century sonata from a written score totally new to her, relies on familiarity with sonata form, piano music of the period, and many other typifications to begin to make sense of the piece and express an understanding in performance. The pianist brings out the particularity of the music by relying on a large stock of culturally developed knowledge concerning the structure, sound, and movement of music of the sort she is performing as well as on the embodied technique of piano performance. The more the pianist locates the music within its traditions, the more tools for understanding and interpreting she has, as well as for noticing and bringing 
out what is thematically new or striking in the piece. Thus the pianist with familiarity can heighten the particular character, sound, or pleasure to be found within each piece. The more, in fact, performers work on music of similar types - whether of the same decade, genre, and nationality or of the same composer, or even period in the composer's life-the more precisely she can develop an interpretation in and against the typical motifs and organization of these more finely tuned types. Even increasing familiarity with that one piece of music and its performance by different artists creates a most local type within which theme and variation, foregrounding and backgrounding can take on local shape against expectations and the environment created across the moments of the piece.

Equally we can say in any music tradition every learning of novel pieces relies on training within the tradition, learning its types, organization, techniques, thematizations, and structuring of the sound space and temporal experience. The more the performer gets to know what the music sounds like and how to produce it, of course the better she can play the most typical of music, but even more, the more refined, complex, surprising, pleasing and interesting effects she can bring to being within the type of music. The typification is not only in the overt forms that can be described abstractly, but within the complex realm of practices of listening, understanding, and performing.

The listener of each kind of music also engages in a process of learning to attune to this music, learning what to listen for, how the sound is organized, what the formal rhythms are (as might be described in a music appreciation book), but also in actual embodied experience of listening to the musicactually immersing oneself in the sound of the kind of thing that is going on, and letting that hearing shape one's consciousness. In that process one becomes in a sense a typical listener, an anonymous listener, but a fully formed anonymous listener, able to hear the music with all that is produced following the motives and motifs and intentions put into the music by the composer and performer. The more one learns to listen to a kind of music, and a particular piece, the better able one is to experience a special moment, different and evocative the way no other moment has been, deeply interesting in its particularity, even seeming to evoke one's own most personal of internal sentiments and meanings.

The result of this production and listening of music by the culturally knowledgeable musician and listener is the joint attention over a period of time-not only the externally clocked time of performance, but over the internally experienced time of the music. Indeed, within the range of variations of attunement and experience and knowledge an entire audience can share these moments of attention and the shared sensation of the passage of consciousness 
through time. This is as true of villagers gathered in a temple in Bali to hear a monkey chant as it is of a modern American teenagers attending a rock concert or of King Friederich listening to Bach's latest organ invention.

It is not a far analogy to apply Schutz's analysis of music to literacy, which also calls for mutual alignment to produced meanings and the giving over of consciousness to performances that draw on detailed knowledge of typified realms. The more refined the writer's and the reader's knowledge of the communicative domain, the greater the potential for refinement of meaning and experience. One of the great powers of literacy is the handing over of our consciousnesses to meanings evoked by others, the re-creation of others meanings in our own minds. Nonetheless, some differences between music and writing might limit the analogy. First, music as an activity is often taken as an activity in itself. We listen to music to enjoy it, to appreciate the performance, to give ourselves over to it (though it may be secondarily embedded in other social occasions, whether of personal relationships or nationalist bonding, where participating in a musically induced state of consciousness has implications for participating in other systems and activities.) While some reading is as purely for enjoyment as listening to music, our reading is often more subordinate to other projects we may have, whether keeping up with the news as part of political engagement or looking at consumer information to decide which car to buy. Thus in reading we are less likely to be entering into an autonomous area of activity whose meanings are primarily embedded within that activity; rather we are likely to be engaged in any aspect of life, from health or the spirit to work or recreation. As we read in those domains, words will call on our knowledge and experiences of those domains and will expand and reconfigure our understanding of those parts of the world, whether of law or insurance, of geology or international relations, of entertainment celebrities or personal relations. Even in reading for enjoyment we engage our knowledge of the world and its domains, and our thoughts and feelings relevant to those domains.

Writing's complex interplay of typification, social and cultural knowledge, experience with the world, and the making of individual meaning is powerfully at play in the experience of poetry, where the common linguistic medium is precisely chosen and shaped to evoke powerful personal meanings and emotions - such as the way a Shakespearean sonnet in its well-crafted words become the container for the reader's individualized sentiments of loves and longings as well as perhaps memories of specific moments and relationships we associate with it. Equally, such seemingly different languages as that of law or scientific specialties, evoke experiences of the social and historical worlds in which the individual develops and acts. 


\section{SCHUTZ, BERGER, AND LUCKMAN AND THE SOCIAL PRODUCTION OF THE EVERYDAY}

Schutz provides us a philosophic understanding of the relation of individual consciousness and meaning to socially patterned structures of meaning. Although Schutz started out with the problem of specialized economic behavior, he generalized to the everyday world which we are socialized into before our reflexive understanding develops. This everyday sense of what is natural is so deeply habitual that we don't realize the social understandings and practices that create it. His students such as Peter Berger and Thomas Luckmann who co-authored the widely influential Social Construction of Reality (1966), took the inquiry in a more sociological direction, examining the processes and patterns of social organization that create structures of individual consciousness and individual's perceptions of their lives and themselves that in turn influence actions.

They have since pursued the practices of forming life accounts, by examining what reconstructive genres, where individuals create public memories of events that have allegedly previously occurred. Gossip and story telling are reconstructive genres, and we may note have some relation to fictional literary narratives. Bergmann has explored genres of gossip at some depth in his book Discreet Indiscretions (1993), where he notes that gossip is filled with ambivalences, denials and ploys to cope with its dangerous violations of the public and the private, the discreet and the indiscreet, the taboo and the envied, the intimate and the condemnatory, and other social boundaries. In doing so, the social genres of gossip create a special recognizable social discursive place where gossip occurs and into which gossip partners must make entry, even as the person gossiped about must be excluded. Nonetheless, the creation of this holiday from usual social norms reconfirms the speaker's commitment to everyday morality about which the gossip so carefully plays. Moreover, gossip creates accounts that evaluate everyday behavior and to which the gossipers thereby make themselves accountable. Here we see the importance of genres for formation of attitudes and we see how social relations and groups are built around the moral recounting of daily life. These are issues of some interest for the practice of literature.

More broadly, Luckmann (1995) has specifically drawn the connection between genre and the construction of daily life:

The elementary function of communicative genres in social life is to organize, routinize, and render (more or less) obligatory the solutions to recurrent communicative 
problems. The communicative problems for which such solutions are socially established and deposited in the social stock of knowledge tend to be those which touch upon the communicative aspects of those kinds of social interactions which are important for the maintenance of a given social order. ... Different societies therefore do not have the same repertoire of communicative genres, and the communicative genres of one epoch may dissolve into more "spontaneous" communicative processes, while heretofore unbound communicative genres congeal into new genres....

At any particular time in any particular society the repertoire of communicative genres constitutes the "hard core" of the communicative dimensions of social life (Bergmann, p. 182; see also Bergmann \& Luckmann, 1995).

Günthner and Knoblauch (1995) further refine the idea of repertoire of communicative genres to a communicative budget which attends not only to the available range of genres, but how these genres are socially distributed (according to characteristics such as gender, caste or office; according to institutional domain such as gender or religion; and according to heterogeneous groupings such as family and leisure groups). The communicative budget gives concrete form to Bourdieu's more general notion of a linguistic field (1991), specifying the kinds of linguistic acts available to the various participants, thus shaping their roles and forms of interaction, and contributing to the formation of their habitus.

Schutz's phenomenology also stands behind other recent micro-sociological examinations of social order created by mechanisms of meaning and sensemaking in concrete interactions, including ethnomethodology, conversational analysis and Goffman's presentation of self, as we will examine in later chapters. In all these approaches social structure can be seen as concretely enacted in micro-events created by individual agents, acting in typified circumstances. Genre thus can be seen as a way of bridging traditional macro-sociology of roles, norms, and classes with more recent micro-sociology, which in looking at the details of concrete interactions has been skeptical about traditional macrocategories that are not easily identifiable at the level of unique encounters among individuals. ${ }^{4}$ Genre provides a means for individuals to orient toward and enact

4. Conversational analysis, for example, in trying to give a precise empirical grounding to social observations, has tended to set aside any abstractions about context, event, or organization that individuals may bring with them to situations. They have attended to the smallest details which might indicate a kind of syntax of interaction, with most attention to the way in which 
situations in recognizable ways with recognizable consequences. Genre thereby establishes a concrete mechanism for structurational theories, that suggest that social structure is constantly remade in every interaction which reenacts ordered relations (Giddens, 1984). Luhmann $(1983,1995)$ has further suggested that society exists in the communications that go between individuals rather than in the aggregation of individuals, who always act as individual agents, and thus social structure is to be found in the structuring of communications, which in turn structure social relations.

Schutz's phenomenology provides a philosophic means for understanding how we achieve mutual orientations and attitudes towards meaningful utterances and their contexts, giving shape to our motives. But just as it has been the task of sociologists to see how these communicative practices concretely shape social relations and give rise to social structure, it is the task of specialists in rhetoric and writing to understand the production, reception, and use of texts within concrete social circumstances in order to produce specific socially shared meanings and knowledge. Because text production and use are so deeply enmeshed in the formation of individual and group consciousness, Schutz provides us a fundamental means for considering the ways in which texts orient our minds towards social worlds of action.

To put it another way, texts are vehicles of articulating meanings within social spaces, externalizing inwardly conceived impulses and relationships into social actions to influence the consciousness of readers through the meanings conveyed. The typifications and social-symbolic understandings that are brought to bear in the course of externalizing and internalizing meanings are strengthened (in both a neural network sense and a personal identity sense) in the course of their active rehearsal. Each time we invoke sets of social understandings, we become that much more engaged with, oriented towards, and committed to those social arrangements, practices, and forms of consciousness being rehearsed. We turn them into stronger social and phenomenological realities. We strengthen the reification. This is a view consistent with and elaborating Vygotsky's understanding of the role of language in shaping mind and regulating activity (see also Russell, 2010). Through participation in social spheres of discursive action, attending to the objects of that sphere in the ways appropriate to that sphere, we develop our minds and modes of thought in socially mediated ways.

turn taking is negotiated. However, in examining how people manage to gain the floor for longer turns, Schegloff (1996) considers larger recognizable turn units—which are something like recognizable genres. If someone is telling a joke, you know to let her continue until the punchline. 


\section{ACTIVE SOCIAL SYMBOLIC SELVES: THE PRAGMATIC TRADITION WITHIN AMERICAN SOCIAL SCIENCE}

American pragmatism developed contemporaneously with the Vygotskian activity theory tradition and the phenomenological sociology tradition, and has many affinities with both. Historically, there were some connections among them: all had common roots in Hegel; Vygotsky read and cited James and Dewey; Simmel and Husserl read and cited James; Thomas and Park did dissertation work under Simmel in Germany; Schutz interpreted James's theories (see Joas, 1993). Nonetheless, each pursued its own path. Each developed different dimensions of a picture applicable to understanding what it means to write; yet, the pictures they draw can be usefully brought together to create a multidimensional portrait. The connections will also reveal why researchers and theorists from these several traditions have been increasingly finding each other's work of interest.

The Soviet Vygotskian interests are in psychology, creating an understanding of socializing individual development and the development of meaning and consciousness in relation to the publicly available activities and mediating symbols and tools. The European phenomenological tradition highlights the formation of socially evolving typified meaning systems that help individuals make sense of situations and frame individual actions which others can make sense of through the socially available repertoires of types. This phenomenological perspective forms an alliance with Wittgenstein's (1958) ideas of meaning representations being parts of active forms of life.

American pragmatism, rather than looking inward to the mind, locates meaning and communication in creative problem solving by people responding to the changing contingencies of their times. Starting as a philosophic response to a crisis in traditional meaning systems, pragmatism directs our attention to detailed historical and social knowledge of the conditions and perceptions of groups that give meaning to their orientations and choices. Philosophic pragmatism has influenced the formation of a number of the social sciences in North America, leading most directly to social psychology and symbolic interactionism in sociology. The approach methodologically 
fostered ethnographic sociology that attempts to recover the meanings and intentions among people acting within particular social systems. Pragmatism also influenced the formation of anthropology and linguistic anthropology, was instrumental in progressive social activism, and was in dialogue with interpersonal psychiatry. Each of these disciplines has important things to say about the act of writing, the social forums and activities within which we write, how we make sense of our own and other people's writing, and the relation of writing, emotions, and identity.

\section{PHILOSOPHIC PRAGMATISM}

Pragmatism has its roots in philosophic crises of the nineteenth centuries and many scholars still see pragmatism primarily as a philosophic movement, to be discussed and evaluated within philosophic discourse. The founders of pragmatism, Charles Peirce, William James, John Dewey, and George Herbert Mead all at some point found their employment in philosophy departments. For all of them, the social upheavals of the latter half of the nineteenth century in the United States - the Civil War, industrialization, and urbanizationupset the sense of continuities and verities which underlay North American values, belief, and security in the world (Menand, 2001). While pragmatism went into an eclipse within philosophy departments in the middle of the twentieth century, it reemerged in the closing decades of that century as a way out of epistemological battles fought on the shifting, alleged border between modernism and postmodernism (see, for example, Rorty, 1979).

Insofar as pragmatism is represented as a way out of philosophic and theologic dilemmas - issues that certainly motivated Peirce, James and Mead throughout their careers, and Dewey for the earlier part of his career-it is caught up in complicated arguments and semantic wrangles within those highly conceptual domains. The irony in being caught up in theologic and philosophic terms is that pragmatism suggests there is no ultimate epistemic authority to be found in theologic or philosophic abstractions. Rather pragmatists see these endeavors, as they see all human endeavors, as emergent historical creations to serve human needs. A further irony to being shackled to existing philosophic and theologic terms is that pragmatism values exploration and sees the human practical and intellectual worlds as experiments. It therefore reflexively encourages reaching towards ideas through only partly formulated and unstable terms. Dewey, James, Peirce, and Mead have all been accused of slippery terms. Moreover, each is highly exploratory in different directions, taking the starting point of a loosely related set of orientations and applying 
them to a range of projects and problems. Each of these versions of pragmatism has its particular set of concerns and attempts its own form of argument. None has a strong motive to create a stable, coherent set of rock-solid claims, in part because the pragmatist approach suggests the futility of coming to knowledge that rises beyond human time and situations. Pragmatism as a philosophy is a loose and baggy universe.

\section{PRAGMATISM AS A PERSPECTIVE FOR SOCIAL UNDERSTANDING AND ACTION}

My interest here is not, however, in the philosophic arguments that go under the banners of pragmatism and anti-pragmatism, whether at the beginning or the end of the twentieth century. Rather I am interested in pragmatism as the source of a number of fairly straightforward premises that underlay many of the developments in American social science, which are directly applicable to literate rhetoric. Although rhetoricians, in their millennia-old skirmishes with philosophy, are ever tempted to see philosophic issues as their own (see for example Gross \& Keith, 1996; and Harris, 2005), philosophy can be applied to rhetoric in a more practical way through the visions various philosophies propose about who we are, how we communicate, and what the consequences of communication are. In particular, pragmatism orients our attention to concrete human actions and communication as action, formative for human thought, interaction, and social organization.

The founders of pragmatism and their early associates were engaged with forms of practice and research in the social sciences and social services: Charles Peirce with language studies; William James with psychology; John Dewey with politics, psychology, and education; Jane Addams in the formation of settlement houses and community development; George Herbert Mead with sociology; and Oliver Wendell Holmes, Jr., with the law. Dewey and Mead particularly were influential in fostering the climate within the new University of Chicago (opening in 1892) that was to be so generative for all the American social sciences, even though the strongly identifiable "Chicago schools" in only some of disciplines (notably sociology and anthropology) showed direct affinity to pragmatist understandings. Dewey's prominence in education and as a public figure also brought his ideas into a general climate of understanding under the banner of progressivism that far exceeded any clearly defined lines of direct influence, and indeed a number of Chicago departments became known for their community involvement, from the time of Jane Adam's Hull House onwards. 


\section{THE DIFFERENCES AND COMMONALITIES OF EARLY PRAGMATISMS}

Although historians of philosophy debate who was the proper founder of pragmatism, pragmatism was already a climate before it was formally articulated by Peirce, James, Dewey, or anyone else. The facts that Dewey was able to gather so rapidly so many like-minded people when in 1894 he became chair of the department of philosophy at Chicago and that he and Mead were able to establish so many interdisciplinary connections suggest just how fully their orientation was compatible with many then in the U.S. academic world. Pragmatism has been reasonably said to grow out of interrelated developments in nineteenth century US: the forging of a new society, great opportunities for action and social change, belief in individuality and optimism, technological transformation, economic growth which was bringing about new social roles and forms of organization, the many religious and communal experiments, the great depredations that came along with the assertion of the new economic power, the newly made social wrongs that needed so visibly to be righted, the many immigrant cultures mixing in new cities, and the practical orientation of this society on the make. When new more democratic universities arose in the post-Civil War American Midwest (such as the land grants under the Morrill Act and the independent University of Chicago) they confronted the domination of European thought with a research culture tied to practical needs of a rapidly growing society instead of reproduction of social elites. The intellectual conditions, needs, and opportunities of the time made a pragmatic orientation easily imaginable and attractive.

The various philosophic issues, research, and practical projects, and spiritual and ethical concerns that gathered in and around pragmatism drew on a cluster of related premises:

- that human knowledge and belief depended on the humans who were making them;

- that human belief, knowledge, and perception were always interpretive;

- that the interpretations come not only from the social and historical position of the person, but from their engagement in projects to satisfy their needs, desires, and value-laden senses of fulfillment;

- that these projects were shaped by perceived problems and sought solutions;

- that these projects and the perceived problems were always necessarily social and material;

- that ideas, discussions, and reasoning developed within situations appearing as problematic; 
- that values, beliefs, knowledge, perception, interpretations, and identities arose out of material and social projects, and were consequential for their solution;

- that there were ethical choices to be made about projects, based on the kinds of consequences that we might project flowing from those choices.

Thus, pragmatism saw history and knowledge as emergent and never fully absolute or predictable, but rather exploratory and creative. These views have significant consequences for how we understand how people communicate, how they use language, what language in fact is, and how language influences how individuals and groups develop. Writing, in particular, provides new potentials for creative communicative, enduring and transportable linguistic artifacts, and restructuring of group relations.

\section{PEIRCE'S SEMIOTICS WITH INTERPRETATION}

Charles Peirce, among the founding generation of pragmatists, looked most directly at language and semiotics, making some first steps towards articulating the implications of a pragmatist view for language and language use. Most importantly, he recognized a major role for the interpreting speaker and interpreting hearer in the meanings conveyed by communication, rather than assuming meaning was immanent in an abstracted language system (Peirce, 1958). It is people who attach meanings to experienced worlds and issues of concern. This recognition of the importance of interpretive processes might lead to an investigation of how differences in individuals and groups of individuals might influence the bases and procedures of interpretation within specific situations (potentially a psychological, sociological, anthropological and even historical inquiry). Peirce, however, chose to seek clarity through a semiotic taxonomy of the relations among signs, objects, and interpretants (that is, interpreted meanings), a taxonomy that he kept adjusting throughout his life. His account does suggest some of the instability of semiosis, as meanings are dynamically produced through interpretation, which is potentially infinite; nonetheless, he seems to believe that this instability can be contained by establishing an abstract philosophic vocabulary about the relations of signs, objects, and interpretants. His taxonomy does not provide any specific leads about how we might inquire into the psychological or sociological variables of meaning making and interpretation. In not pursuing the motives of the individual nor the development of the individual in satisfying needs within the social and material worlds, Peirce leaves us with a mystery of the individuality of interpretation creating indeterminacy of meaning, with no way to get back to the sources, needs, and mechanisms for meaning making. Yet it is these underlying 
forces that drive all utterances including writing and lead to the proliferation of new texts, new genres, and new fields of literate interaction. Pierce, therefore, does not yet provide us with an understanding of how and why people use language to produce the creative inventions that are at the heart of the pragmatic worldview.

\section{JAMES'S PSYCHOLOGY OF EXPERIENCE}

William James in his psychology does, however, provide first steps towards a way of understanding individual sense-making, choice-making, and language use. His psychology is founded upon experience rather than separate sensations, systematic thought, or a rationalized view of language as a stable meaning system. He presents people as embodied creatures acting in the world, with horizons of interests, knowledge, and attention. People he sees as responding in the moment to situations driven by desires and immersed in feelings (1890, 1 , chapter 10). Thus people's ideas and perceptions are typically vague in a philosophic sense, only sharpened and clarified insofar as it is necessary to act in the world $(1890,1$, p. 218). The implication is that use of language is only precise as it needs to be-perhaps to elicit cooperation, or to sort out action paths, or whatever other purpose is at hand. Language does not have any meaning apart from people's uses and uses are only precise as the situation and interaction with others require it to be. Whatever degree of communal precision and clarity of language that does exist results from a communal history of developing linguistic practices. Individuals then each have a developmental history of linguistic practices in interaction with members of the community, within the accomplishment of those tasks available and motivating within that world of practice (James, 1912). Those specialized domains seeking clarity of sensation and reasoning, such as science or philosophy are equally driven by our sense of the problematic and are limited by our stance of perception and action in the world, even as they rely on written language to reflect on, sort through, and evaluate claims.

\section{DEWEY'S THINKING ABOUT PROBLEMS}

Dewey pursues this situated action perspective by arguing our perception and reason are based on our sense of being in the world and the projects we pursue as creatures in the world. We do not have a pure, disengaged consciousness; our stream of consciousness is not random. Our powers of consciousness only arise as a means to reflect on and resolve situations where we perceive a problem 
(1896, 1910). Communal thought and action he sees equally as arising from perceived problem situations that are seen as needing response. While James finds in vagueness a space for intimations of religious experience outside the realm of science, Dewey finds in vagueness a creative force for the constant invention and change of human experience and increasing clarity, as we address perceived problems and try to look more intently and coherently at those things we sense as problematic (1910). Thus Dewey and his followers tend to be politically and socially progressive, insistent that individuals and societies address problems and seek improvement of the conditions and practices of life. They believe that in resolving problems, individuals and societies will grow toward more satisfying modes of existence.

Dewey himself was so forward looking, ready to seek social change to resolve felt difficulties in society, that he spent less attention than he might have on the particular forms and relations embodied in existing conditions, the history of how they got to be that way, or the mechanisms by which social, economic practices occurred. In retrospect he seemed to have a political naïveté about the degree and speed at which change could be brought about and suffered a chastening and withdrawal from activists (Feffer, 1993). Similarly in his own work there is little detailed analysis of the social mechanisms of the current world or the historical processes by which current problems and tensions emerged, although he often called for such analysis and emphasized the importance of studying history in the schools. He also talked about the importance of knowledge, existing disciplines, and human accomplishment as basis for building on and transforming. As the progressive education movement developed he was distressed to find that there was not always adequate attention to the available resources already developed by humans, and he often had to explain in later years his commitment to discipline and knowledge. However, his own discourse provided few examples of how that integration of knowledge of the past and new action might occur, and his own advocacy for change rarely included such close attention to the complex of things that have already come into being. Nevertheless, he saw that the motive for action, perceived problems calling for solution, and the felt discomforts of life all came from the social understandings, practices, and histories that informed people's motives and views of situations.

\section{DEWEY'S LEARNING THROUGH ACTIVE EXPERIENCE}

Because Dewey saw education as forming the individual with the skills, knowledge, and disposition to participate in activities and problems to be solved 
in society, he saw education as the most important site for social intervention and contribution to society. Dewey saw learning as motivated growth arising out of the situation and experiences of the child, which educational projects needed to speak to if they hoped to enlist the most active engagement of the child (1897, 1947). Thus he argued for a substantive connection between the activities of the school and the life in the community from which the students came. He and his followers advocated project-based education addressing the perceived needs and opportunities of the time and place (Kilpatrick, 1951; McMurry, 1920; Tanner, 1997). For Dewey, in education as in life, the key to activity, growth, and accomplishment is motivation, for knowledge and growth and projects have to speak to the possibilities, opportunities, and needs in front of one.

If motivated agency is located in the possibilities one can identify in the moment, and successful agency requires a responsiveness of the material and social situation, then understanding one's situation is an appropriate object for educational inquiry so as to be able to evaluate potential action. Further, if learning depends on motivation and perceived problem - that is, felt need for action-then learning occurs within the tensions of perceived problems. The learner and the researcher are driven by the urge to intervene and transformno matter how much the inquirer distances him or herself from the object studied through canons of objective study, under a belief, often well-founded, that to act too soon is to act with inadequate understanding. Yet, we should not mistake the distancing of responsible inquiry for total disengagement from future benefit. Rather Dewey would have us think of a deferred engagement (1896). With a total disengagement or perfect objectivity, objects lose all interest, value, and desire.

Dewey's views on problem-solving, agency, motivation, and learning are directly applicable to writing, and in fact have been repeatedly applied over the last century in various inquiry, project, and discipline-based writing pedagogies and the thematic orientation towards authentic writing tasks which engage students' interests and concerns (Russell 1991, 1993). Once the connection of personal engagement in meaningful problems is made, the task becomes an expression and development of the self, even if no overtly personal material is discussed and even if the writing task seems objective, technical, or professionally cool. After all, no one is as passionate about statistics as a statistician. Outside formal educational contexts, Dewey's construction of learning through problem-solving means that writers continue to grow as writers through the various challenging tasks they take on throughout their lives in the domains of importance to their lives.

Dewey's educational philosophy met two kinds of criticism: on the conservative side from those who felt that education should pass on the 
tools of knowledge already developed and on the progressive side who saw him providing a rationale for accommodating people to the existing way of life, preparing them for factory and office work of industrial corporatism. In response to the conservative critique, Dewey regularly insisted on a middle way, respecting and passing on the historical legacy, but always harnessing that to the needs and motives and situations of people, for that was the very mechanism by which people were motivated and grew. In response to the progressive critique he argued that effective and meaningful change must be situated in the reality of situations and the problems situations present. Accordingly, he believed change was evolutionary within the continuing forces of life and that there is no absolute of value or of practice that could warrant a radical rupture from current ways of life.

Dewey's principles stood behind his collaboration with Ella Flagg Young in creating the University of Chicago Laboratory School (Tanner, 1997). Young was to continue to actively shape education on these principles as principal of the Chicago Normal School and later Superintendent of the Chicago public schools, and eventually president of the National Educational Association.

\section{THE PROBLEM OF LIVING WITH OTHERS: MEAD}

George Herbert Mead, a colleague of Dewey, both at Michigan and then at Chicago, also saw how people addressed the problems of life as core to understanding and improving society. Mead aimed to understand how individuals came to see themselves within the social relations and social understandings of their times, particularly through learning of gesture and language. In coming to learn to use meaningful symbols, the individual has to be able to anticipate how others might perceive the symbols and perceive him or herself in using the symbols. Skilled communication requires that a person needs to learn to anticipate how others might take meaning from any word or gesture, and how that meaning might prompt response and consequent actions. Further, as a person observes the response of others to comments and behaviors the person gets further data to help project how one is seen by others and thus understand the social self one is projecting. That is, in learning to use meaningful symbols, the person learns to take the perspective of the other, both particularized others and a generalized other. This perception of how others see one forms a sense of the self. Mead sees taking the part of others as part of learning to be in society and as a major theme in children's play. Thus, in learning to live within society we learn to see ourselves and judge our own behavior as others might - a process that might be considered internalization 
of social norms. Yet, since we are constantly solving novel problems in novel circumstances and our motives extend far beyond just fitting in, or being secure our learning to take the part of the other hardly limits our creativity and originality. It simply maximizes the possibility that others will understand and cooperate with us without misunderstandings that lead to violence or other forms of social control (Mead, 1913, 1934, 1936).

This formation of the self and articulation of identity within the social field applies precisely to writing as we come to understand the force and meaning of our writing in the presence it creates for others. The process of seeing what sense others make of our writing helps us understand what our texts do and do not accomplish and what social presence we are creating for ourselves through our texts. The response of others also gives us information about how we can revise or reshape our statements, or create new statements, so as to bring that presence more in line with our desires. Simultaneously we become committed to the intelligible presence we have taken on in our writing. We can examine our texts apart from ourselves and learn to take the part of the other in evaluating and improving our text as we become more experienced writers, with less naïve attachment to our first sketchy formulations. Yet we also come to understand that the texts represent us to others and therefore they become an extended part of ourselves. Especially as we write to people at a greater temporal, geographic, and social distance from ourselves, to create an intelligible presence we must use the common language recognizable to others, but through that language we create the individuality of our statement.

Just as Dewey worked with the Laboratory School, Mead worked with Jane Addams in Hull House. Addams (1997) viewed the settlement house as a way of being of an entire community to change people's view of themselves and capabilities to act in society. It was aimed at social change based on people being empowered to identify and act on problems in their lives through jobs, education, and access to social services and other forms of support. The settlement house in many ways was the concrete realization of Mead's thinking about the formation of ourselves as actors in society.

\section{MEAD IN RELATION TO OTHER TRADITIONS}

In some senses Mead was following on the heels of the Scottish moralists (such as Francis Hutcheson, Dugald Stewart, and Adam Smith. Smith's Theory of Moral Sentiments (1986), in particular, described the conscience which guided moral behavior as a perception of how others might perceive and evaluate one's actions if others were to have the full knowledge of the situation as oneself (or 
see the situation as one perceived it). Mead apparently was familiar with Smith's writings and had written an undergraduate paper on Smith while at Harvard ( $T$. V. Smith, 1931; see also Blasi, 1998). Mead, like Smith, recognizes that no two people have the same set of experiences or knowledge so they never quite see the circumstances exactly like another-thus there is always an individuality of judgment, evaluation, decision, and action. Mead, like Smith, equally recognizes that one's judgment, perception, thoughts, and capacities are very much influenced by things like education, occupation, class, cultural background, family organization, prosperity, and historical moment-and these influences might conflict with an unencumbered understanding of one's interests.

In the years between Smith and Mead, Hegel and Marx also noted the influence of social ideology on beliefs and actions. Marx sees the socializing impulses to be so strong as to potentially blind oneself to one's needs, desires, and impulses in favor of fitting in with the reigning thoughts and formulations, or ideology (Marx \& Engels, 1971). Smith similarly recognized a tendency of people to admire hierarchy and the perceived power of the dominant class, which can obscure perception of one's best interests; nonetheless, Smith suggests that the individual is in the best position to know what he or she needs and wants and what the local opportunities are, if they are freed to make unencumbered judgments in one's own interest. Mead characterizes this tension between social belief and individual perception of interest in a different way. Mead sets the socialized $m e$ in tension with an impulsive $I$ (like Freud's id and ego) which regularly surprises oneself by its spontaneous assertions of desires and perceptions, with a result that individuality and agency cannot be fully suppressed. This agency sometimes acts within the bounds of the socialized self, but always is ready to push beyond the bounds of what one might anticipate others seeing as acceptable or intelligible (1934).

Thus both Smith and Mead see great variety within the socialized selves of any time and place, arising from the variety of positions, experiences, and spontaneous expressions of interests and desires. Consequently both saw institutional and other organized aggregations of activity as complex, embodying the multiple motives and activities of participants. Mead, along with other pragmatists, was particularly interested in the creativity of problem solving, as each person brought new resources, perceptions, and problem definitions to situations to remake the social order. Smith, on the whole, was more cautious, even pessimistic about change, in light of what he saw as peoples' desire to stick to older ways and to respect the elites who had an interest in maintaining arrangements that granted them privilege.

Mead's recognition of the role of language processes in the formation of the socialized self and the mind, however, clearly sets him apart from the 
Scottish Moralists or Marx, and puts him nearer to Vygotsky. Mead sees the mind formed in learning to make meaning with and for others, as one sees the effects of communications on others. For both Mead and Vygotsky, though in slightly different ways, self and mind are products of language use in society. For Vygotsky and Mead, speech is a form of act, not a disembodied meaning or truth, but always formulated in action, as part of action, and therefore acting in the world. Thus the meanings we develop in interaction and the thoughts we ponder are saturated with the shades of prior action and the anticipations of new actions. The formulas of unconsidered, unproblematic, habitual utterances are part of those activities we think we know so well that we don't have to think about or contemplate-all we need to do is produce the prefabricated words that carry out the old solution (though we may well find ourselves wrong, or we might do better if we stopped to think afresh). Thoughtful speech-the words that make us think or that we feel we need to think about before we speak-is a creative action prompted by a perceived unresolved problem to which we are responding (Blasi, 1998, p. 167; Mead, 1934). Writing is paradigmatic of thoughtful speech as it readily affords planning, examination of alternatives, choice-making, and review and revision.

This problem-solving activity, however, does not necessarily put us in the realm of pure individualistic utilitarian instrumentalism as a number of the critics of pragmatism have asserted, for Mead's communicative mechanism of learning to take the part of the other draws us into social relations as part of our participating in the world. In learning to talk with each other we learn about common values and norms. We develop social consciousness and orient towards the maintenance of the group. We learn our own interpersonal needs and the ways other persons enter into our own needs. We learn of the importance and power of social bonds, and we learn to recognize those who think well or poorly of us - and adjust our behavior and relations depending on how we evaluate their opinions. We recognize whom we can talk to about what, with what kind of support and seriousness. Obtaining and maintaining the positive opinions of others, particularly those on whom our daily life depends and who are partners in our daily life and daily needs satisfactions, becomes itself a social motive-as elaborated by Harry Stack Sullivan, discussed later in this chapter. Similarly we learn to enter into the larger orders of publicly organized systems of meaning and community, such as investigated by Durkheim. While Mead does not pursue this line of reasoning, and Durkheim even sees pragmatism as threatening to obscure the social production of values by being too individual and instrumentalist, there is no necessary incompatibility between Durkheim's more macrosociological considerations and the ethnographic tradition, as 
numerous ethnographic studies have since recognized, starting with RadcliffeBrown (1922, 1931) and Mauss (1922) (see also Joas, 1993).

Further, typification processes, as discussed in the previous chapter, allow individuals to build senses of more or less generalized others who operate within recognizable systems of typifications (for discussions of Mead's relations with phenomenology see Natanson, 1956; Pfuetze, 1954; Rosenthal \& Bourgeois, 1991). Micro-processes of self-recognition in interaction thus have the potential to scale up into larger social orders, particularly as the interactions are mediated by the more enduring and transportable means of writing (as will be examined in Chapter 6, see also Bazerman, 2006). Indeed, as Joas (1985) discusses, there is no necessary incompatibility between Meadian processes of self-formation and certain forms of structural functional sociology, which often are built upon mechanisms of orientation to the other, such as role theory and reference group theory, as to be discussed in Chapter 6. Indeed orientation to others is one of the areas that there is much cross citation and cross acceptance of findings between symbolic interactionists and structural functionalists. We may indeed see in such hybrid researchers as Erving Goffman, discussed in Chapter 7 , the power of such conjunctions of micro and macro considerations around phenomenologically drawn individual problem solving.

\section{MEAD, CHICAGO SOCIOLOGY, AND SYMBOLIC INTERACTIONISM}

Mead's understanding of the formation of the social self is the direct antecedent of those branches of sociology that emphasize meanings people attribute to situations, themselves, others, and actions. Social psychology and symbolic interaction see themselves as direct heirs of the Meadian tradition (see Bulmer, 1986; Faris, 1979; Matthews, 1977; Tomasi, 1998; but Joas, 1985; T. V. Smith, 1931 and others argue that far too much has been made of Mead's influence). As we will see in the next chapter, other concepts of other aspects of American sociology are grounded on Mead's view of the socialized self-perceiving its own position through the eyes of others, or at least what it can glean of the eyes of others. Participants' definition of situations (which involves their definitions of selves and others within particular action contexts) has become a key element in most programs of empirical and theoretical sociology.

As both Blasi (1998) and Joas (1993) point out, Chicago sociology has had a widespread, diffuse but pervasive approach on interpretive, qualitative and 
empirical sociologies that examine the individual's perceptions of self within social groupings and activities. The sociology department in the University of Chicago dominated American sociology in the field's formative years. Prior to the Second World War that department produced the majority of PhDs in the field and many of the most prominent. The American Journal of Sociology (founded at Chicago in 1895 and still there) was until 1921 the only major journal in sociology and remains one of the dominant journals of the field. Chicago sociologists were instrumental in founding the American Sociology Association in 1924, and of the first twenty-five presidents of that organization, fifteen either taught at Chicago or obtained their PhD's there. The relevance for this study is to suggest that many of the assumptions underlying the profession of sociology have their roots in a pragmatic orientation, even though only some schools claim an explicit descent, and others seem to arise from polar theoretical positions.

\section{LEGAL INSTITUTIONS AND LEGAL PRACTICE AS EXPERIMENT: HOLMES}

To the usual quartet of founders of pragmatism, Menand (1997, 2001) adds a fifth: the jurist and legal theorist Oliver Wendell Holmes, Jr., who is known as the founder of legal pragmatism. But his presence in this group is contested, not least by his own followers who rightfully draw many distinctions between legal pragmatism and philosophic pragmatism and further find longdeveloping roots of legal pragmatism within the legal system (see for example, the essays by Posner, Grey, and Luban in Dickstein, 1998). Indeed, although Holmes as a youth did sit at some meetings of the Metaphysical Club 18701872 in Cambridge with the young Peirce and James, and where presumably some proto-pragmatist ideas were discussed (Howe, 1957, p. 152), Holmes did not have kind things to say later about either Peirce or James (Pohlman 163164). Yet there remain some striking homologies between legal pragmatism and philosophic pragmatism, as Menand (1997) argues. Holmes considers law a continuing and changing experiment that shapes all the conditions of our life, just as the philosophic pragmatists consider life and society ongoing experiments. Holmes sees law as a series of uncertain actions trying to anticipate judgments to be made in the future. Law offers no final truths or ultimate principles to Holmes, only anticipation of what might be taken as determinative principle by the magistrate, or future magistrates. Yet history and precedent have created models and patterns that future individuals are likely to adhere to, 
particularly as they themselves are held accountable by others to the common body of precedent.

Situations and people, nonetheless, are always different; and actions speak to the perceptions of the moment. Further, the future brings unanticipated changes, with new meanings and precedents. For such reasons, to foster experiment, Holmes was a great advocate of the freedom of expression and the first amendment to the U.S. Constitution. He spoke of the importance of the marketplace of ideas and of limiting judicial interference in even apparently unwise actions so as to let experiments to run their course. The law only sets the conditions for social experiment, but does not judge it. Further he was willing to change precedent whose only continuing warrant was that it was precedent, as conditions change and make prior wise decisions irrelevant to changing ways of life. Yet these changes and new experiments are always accountable to the realistic conditions of the new way of life. Experiments have to pay their costs in the marketplaces of life, and judicial wisdom comes in seeing the conditions of life that warrant reevaluation of precedents. All these views are consistent with philosophic pragmatism and the social activism of the pragmatists, though Holmes' politics were more conservative than reformers like Dewey.

Holmes views on freedom of expression to address changing conditions of life and propose new directions for society speak directly to the importance of writing as a mode of reasoning about current conditions, developing new ideas, and arguing for new social arrangements. He provides a warrant for the writing within the public sphere, both in its more traditional forms of journalism, commentary, and advocacy publications and in the newly evolving forms of digital public discussion. From his perspective this work does not simply represent, rehearse, and persuade fixed interests and views, but rather provides the medium for social innovation, new relations, and novel solutions. As we are seeing with new technologies, this innovation goes beyond specific ideas and arguments to the very organization of public community, the kinds of bonds that may be formed among citizens, and the ways they may act individually and as groups to influence public discussion eventuating in policy. But Holmes also points out that public discourse and proposals have to face the judgment of the marketplace of ideas and survive only if they seem attractive and useful to others.

Holmes' views also bear on the more specialized communicative domain of lawyers. As a practical lawyer and jurist, he is concerned with the preparation of briefs and opinions, concrete utterances, concrete symbolic acts, filed on pieces of paper as the very material out of which the law is composed. His organic view of the law invites analysis of the preparation, presentation, and circulation of 
concrete communicative acts in the formation of the law and its life in shaping and adjudicating life actions.

Holmes' views are significant for communication because of the kind of practical influence he has had on the development of one of the overridingly important institutions in the United States, and on the attitudes many people, lawyers and citizens, take toward the law. Thus reflexively, pragmatic beliefs about the evolution of law and society are now built into the views of many lawyers, legislators, and citizens, and have gone into the constructing of legal, governmental and political action, despite others who hold more essentialist views about law. Thus the very way of life studied by American social scientists itself is being built in part on pragmatic assumptions. If law and society are living and evolving as the pragmatists believe, then reflexive understanding of this allows an even greater monitoring, evaluation, and support of these processes, as well as a climate of public belief that favors pragmatic formulations and thus a public ideology of change and experiment. Such pervasive views support a view of legal texts and texts within all domains of society influenced by the law as contingent, situated, and evolving in meaning as conditions change.

\section{PRAGMATIC INFLUENCES ON SAPIR AND LINGUISTIC ANTHROPOLOGY}

The pragmatist approach to understanding socialized individuals, individual and group action, the role of language in individual and group formation, and thought within situated activity also influenced several other parallel lines of development within American social science (See Bulmer, 1986, Chapter 11), including anthropology and linguistics. Edward Sapir, the linguistic anthropologist, is the most direct vehicle of that influence in both fields. After fifteen years as the chief of the Division of Anthropology for the Canadian government in Ottawa, he arrived in 1925 in the small combined department of anthropology and sociology at the University of Chicago, where he remained until 1931 when he went to Yale to found the department of anthropology there. Although his name is now best known through the SapirWhorf hypothesis of linguistic relativity, he had a more moderated view than Whorf about the influence of language on cognition. Sapir was interested in interactions between language form and use and such things as social interests, activity, culture, physical and social environment, thought, and personality (see for example his essay "Language and the Environment," 1912). One could more properly say that, unlike Saussure and other linguists who wanted to isolate linguistic phenomena from social, historical, rhetorical, situational and 
psychological issues in order to make linguistics and autonomous discipline, Sapir wanted to understand language in all its complexity and in its relation to all other aspects of being human, so that language necessarily should be studied in relation to all the other social sciences (see Sapir, 1949). While only some detailed influences between him and his sociological colleagues in Chicago can be concretely traced (Darnell, 1989, p. 214), Sapir clearly shared with them an interest in the activity and interactions within communities, and he provided a means for exploring that interaction through what Mead and Dewey had seen as the key vehicle of social and psychological formation-communicative language (see Sapir, 1935).

Sapir and his teacher Boas are viewed as the founders of linguistic anthropology, and Sapir was one of the founders of the Linguistic Society of America. Linguistic anthropology generally views language as coming to be in interaction, and in doing so becomes a primary vehicle for the creation of social realities and personhood within social-cultural circumstances (see, for examples Bauman, 1986; Duranti \& Goodwin, 1992; Gumperz, 1982; Hanks, 1996). One area of concern for linguistic anthropologists is pragmatics. While the term pragmatics within linguistics has a technical meaning distinct from philosophic pragmatism and should not be confused with it, the study of linguistic pragmatics is based on the assumption that people do things through language, and manipulate the common stock of symbols to interact, form relations, modulate social relations, manage impressions others have, and carry out activities, and thereby make their social world and their own place within it.

Sapir also identifies another point of conjunction for a comprehensive understanding of language practice within a complex social science inquiry. From early in his career he was interested in psychiatry and the formation of personality, and he reviewed books, for example on Freudian and Jungian psychology (for examples, Sapir, 1917, 1923). He saw societies and cultures both as formative of personalities, and formed by people with individual personalities. This interest in psychiatric inquiry took more concrete form after his meeting the psychiatrist Harry Stack Sullivan around 1925, forming a close personal friendship for the remainder of his life. They were to collaborate on many project including conferences, grants, the creation of the interdisciplinary journal Psychiatry and the founding of the Washington School of Psychiatry (See Bazerman, 2005).

Through their friendship, Sapir gained further direction and impetus for his interest in the relation of personality and culture. Sapir was to write a number of papers on the interaction of psychiatry, language and culture (see 1927a, 1927b, 1934a, 1934b, 1938). Sullivan in turn was brought into conversation with the Chicago sociologists, gaining a more concrete sense of the cultural variability 
of life conditions, the ways individuals emerged within social relations, and the role of language in the formation of the individual.

\section{SULLIVAN'S PRAGMATIC INTERPERSONAL PSYCHIATRY}

Sullivan formulated his distinctive theories during the time of his friendship with Sapir. Through Sapir, the political scientist Harold Lasswell, and other acquaintances in and around the University of Chicago, Sullivan became familiar with the ideas of Mead and other pragmatists. Contact with pragmatist theories provided the means for Sullivan's ideas to mature through the remainder of his career and reached their fullest expression in a posthumous reconstruction of his lecture courses The Interpersonal Theory of Psychiatry (Sullivan, 1953). In fact, in that work he discusses the ideas of Mead and his colleagues for several pages (1619). In the Interpersonal Theory Sullivan draws a developmental picture of the child trying to satisfy needs and forming social relations in a social and cultural world. The infant's most fundamental and deepest learning occurs in activity situations with primary caregivers, in which fundamental perceptions of the self and relations to others are formed. In coordinating such activities as feeding, the child learns to integrate in shared events, satisfying mutual needs. Part of that coordination is the sensing of anxiety within the caregiver, which in turn raises anxiety within the infant, for the caregiver's anxiety indicates possible difficulty and uncertainty of outcome. It is out of discovering the emotional spectrum of security, interpersonal unease, and terror in interaction that the child forms a sense of the self (the good me-the range of action and interaction in which I will feel secure), the boundary areas of insecurity and anxiety (the bad me), and those interactions and activities so deeply imbued with extreme anxiety that they are beyond coherent perception and possible participation (the notme-the realm of uncanny sensations). The infant also learns means of coping with or avoiding those situations that raise anxiety. As the child grows into an adult and moves out into the world, filled with people and situations that may challenge an already developed sense of secure situations, a sense of self may expand by experimenting with new ranges of interaction. Nonetheless, most people spend much time in security operations, keeping at bay the anxiety aroused by life's variety.

This model of development is consistent with the pragmatist account of active selves engaged in purposeful need-satisfying interaction. Moreover, Sullivan provides a mechanism for self-formation very closely allied to that proposed by Mead. According to Sullivan, the individual begins to sense a self in relation to the response of others and how one then acts to elicit favorable response. 
Thus the individual is motivated to make interactions go well and anticipate the responses of partner, so as to elicit the cooperation of the other. Sullivan adds to the pragmatist picture the development of the anxiety system that defines the areas of comfort within which the person operates and the areas of discomfort that make it difficult or even impossible to operate. The development of the self system means that one's sense of self formation is saturated with affect, as some behaviors feel more comfortable and secure, while others raise anxiety, and still others are insurmountably aversive, no matter how strong the need, impulse, desire, or attraction. We can then see socialized behavior as a kind of emotion-laden tropism, where one is drawn to anticipated satisfaction by positive anticipation and repelled by the discomfort of behaviors that seem fearfully disruptive of the social bond with partners, based on one's history of interactions. In this pull of needs and desires and push of aversions, one finds a way to act, although the conflict of these forces may cause one to abandon either the need or the security.

Sullivan considers development occurring within interactions over the life course unlike the Freudian view which sees life as irrevocably fettered to the earliest sets of social relationships within the family_primarily with the parents, and barely even with siblings. Sullivan, while recognizing the importance of the earliest relations in learning to coordinate fundamental needs and establishing starting points for trajectories of social participation, still observes that the course of life brings us into important and motivated contact with others. The expanding cast of characters we meet in life presents new developmental challenges, but also allows us to explore new possibilities, and learn new forms of interaction. New relations may also expand the domains of the self that had been bounded by anxiety, as trusted partners help us sense security in situations where we previously had sensed only impending difficulty. While early selfformation and the power of anxiety forcefully lead us to keep replicating early behaviors, that is not necessarily the end of the story.

These complex life trajectories and transformations of the self are driven in part by biological imperatives, but also are responsive social, cultural, economic, and material conditions. Culturally learned patterns of child rearing, widespread taboos and anxieties, and concerns about the good opinions of community and family influence parental interactions and emotions with children. Social arrangements and beliefs affect the range of people one is likely to meet at different junctures in life (at school, in summer camp, on the job) and the patterns, social meanings, and restraints on forming friendships and sexual attachments. Economic opportunities and challenges of daily living focus our energies, turn our attention away from other endeavors, and influence whom we interact with, under what conditions, and for what purposes. 


\section{LANGUAGE AND WRITING AS INTERPERSONAL AND SELF-FORMING}

The relevance of such issues for language and writing should be apparent in that language and writing are media of expansion, learning, and interaction. We use language at the point when our motives meet the motives of others in interaction, always with some challenge and growth as we confront different selfsystems with their divergent understandings, motives, attentions, and anxieties. If communication follows well-worn and familiar tracks that everybody knows exactly where things are going and is perfectly comfortable and secure, the challenges and risks are less. If not, the communication, mediated activity, and learning are rife with possibilities of crossed purposes, misunderstandings, and disjunctions that will lead to ruptures or redefinitions in the communicative situation.

Language is learned in use within a developmental history of relations and anxiety, and the meanings and uses a person finds in language are colored by the emotions of security and anxiety. We all learn to disrupt situations that make us anxious by changing the subject, leading the situation down alternative paths that protect our security, or otherwise being disjunctive of the trouble we sense coming down the road, thereby transforming the situation into one that alleviates our anxiety, even if this means turning away from needs and desires. In the most extreme cases, people who have had consistently unfortunate and anxiety-raising experiences learn to use language far more to ward off anxiety by placating or misleading or distancing others than to communicate in pursuit of the satisfaction of needs. Where anxiety rules, there develops a radical disjunction between, on one hand, a person's needs and embodied experiencethat is, the self one knows as one withdraws from the anxiety of relationshipsand, on the other, the face one presents to the world to keep anxiety at bay. This social learning, of security and anxiety, of self-definition and taboo, of language used to modulate and fend off anxiety, adds another dimension to the social learning of language and interaction to those more typically noted by Vygotsky and socio-cultural psychologists. Additionally, the personal anxiety system described by Sullivan adds another dimension of aversive and mindclouding affect to the goal-shaped affects of motive and frustration noted by Vygotsky (see Bazerman, 2001a, 2001b).

Sullivan was aware of and interested in the work of Vygotsky, though after Vygotsky's death in 1934. Sullivan was instrumental in publishing in 1939 the first translation of the last chapter of Vygotsky's Thought and Speech (Vygotsky, 1939). Sullivan also wrote a commentary on a Vygotsky article in a 1944 volume (Kasinin 1944). While Sullivan sees the origins of the self-system developing 
out of prelinguistic sensations of anxiety, he sees the development of linguistic reflection on the self as extremely powerful in the extensive construction and monitoring of identity and in choice-making as one grows older. In short, language, for Sullivan, as Vygotsky, is the chief tool of reflective action, although Sullivan allows for the interference of security operations to warp the processes of reflective choice making, to provide for indirect or even dysfunctional terms for reflecting on one's needs and desires, and to create distances between one's public expressions and one's inner sentiments. Sullivan, as Vygotsky, gives an account of the development of internal linguistic thought through an internalization process in which language goes sub-vocal and private, a process that Sullivan characterizes as reverie formation (1953, pp. 184-185).

For Sullivan, in addition, language is a means of sharing our perceptions and emotions, validating those individual formations of self, knowledge, and perception. In receptive environments we may have a strong impulse to share how we see ourselves and the world. This sharing of experiences can expand our vision and repair the idiosyncrasies of our experience and personal interpretations. This social validation can impact our constructions of algebra or gravity or the meaning of a John Milton poem as well as our sense of what is socially appropriate to mention to a friend, our perceptions of the emotional reactions of others, and our evaluations of how much risk or pleasure a situation may hold.

Language for Sullivan, as for Vygotsky, is also a means of organizing learning and thought. The developing child, according to Sullivan, as he or she learns language and thereby learns to give shape to thought and coherence to perceptions of the world, moves through stages of prototaxic, parataxic, and syntaxic modes of thought (Sullivan, 1953, pp. 28-29), which are closely congruent with Vygotsky's stages of children's thought and perception 1) prior to the reorganization of thought through language, 2) as the child makes associative connections while using language to organize thought (Vygotsky's sub-stages of congeries, complexes and collections, and pseudo-concepts), and 3) when the adolescent develops coherent systems of language characterized as true concepts, and accommodates thinking to the disciplined and schooled systems of concepts presented through the formal learning of the society-or scientific concepts (Vygotsky, 1986, pp. 110-124).

Sullivan's developmental model of persons learning to act (in large part through language) in fulfillment of needs in interpersonal relations-within the cultural conditions of a time and place and within the particular dynamics of a particular relationships-allows us to consider the role of language development and expanding literacy competence, without being caught up in particular cultural or historic forms of participation taken to be natural. 
We can see language development and literacy development as taking many courses in relation to the historical and social moment, the particularities of the person's prior experience and current motives, and the particularities of the communicative system and situations.

Sullivan, like Vygotsky, shows us an optimistic potential for growth into and beyond the available social and cultural arrangements and activities of one's time and place. Sullivan, however, does not see that growth as necessarily easy, as we must constantly face the anxiety of those things that stretch us beyond that which we are comfortable with. This discomforting anxiety makes it difficult to see what lies in front of us and around us and leads us to want to turn our eyes and thoughts elsewhere, back to the worlds we are comfortable in, where we find a familiar self-definition and perception, in interactions where both ourselves and our partners are secure. Further, in participating in growthoriented relationships, we must not only persuade others of the innovations we create as useful to their own ends, we must address their anxieties, uncertainties, terrors, and senses of where self-security lies.

Although Sullivan never specifically raises issues of writing, he provides a framework of thinking about writing issues as anxiety, formulating and synthesizing knowledge, the anticipating audience, the changing roles for writing as one moves through one's life course, and the cultural variation of literate tasks and its relation to personality and personality development (see Bazerman, 2001b). Sullivan in this way can provide us means to see why writing may be so difficult, why we may resist and struggle with some modes of expression, why we find some audiences easier to address than others. At the same time he provides ways to account for the self-expansion, selfformation, discovery, reflection, and growth that people regularly report as the result of writing. Finally, he allows us to see these processes as within the difficulties and rewards of integrating in social relations with others as part of social projects.

Overall, the pragmatists help us see writing as part of social problem-solving, invention, and evolution. Through writing we address our current needs and concerns and create new arrangements that change our way of life. In doing so, we assert identities and recognize ourselves through what we contribute. We see ourselves reflected through our presence in writing and the presence that writing takes in society. Our challenges, emotions, and difficulties in writing are as much about the place and actions we take in society through our writing as they are about manipulating the technical means and resources of language. Addressing our present circumstances and making our futures means we are never fully sure about where our writing is taking us, how others will see us, and what the consequences will be. 


\section{SOCIAL ORDER: STRUCTURAL AND STRUCTURATIONAL SOCIOLOGY}

The social field on which individuals take action to satisfy needs and desires is created by humans in the relations they form, interactions they enter into, and organizational arrangements they construct or accede to. Further, those human arrangements transform the perceived material field for action through the cultural meaning, attention, and value assigned to the material environment and the work which transforms the material into a built environment of resources and possibilities. To understand how individuals act and interact (and thus how they use spoken and written language), we therefore need to pay attention to humanmade social and material orders. These are not orders hypothesized by social theorists as abstractions, but are rather emergent historical orders, recognized and engaged in by participants.

To be more explicit, built material orders exist because people build them and social orders exist because people act in certain ways with respect to each other. Those built orders and ways of behavior and interactions are dependent on the ways people understand and orient toward the world. Those understandings are based on the individual's life experience and observations, the visible signs provided by others, human-made artifacts embodying social practices and beliefs, and the words used to describe, discuss, evaluate, and regulate the social and material words. Human beings in trying to make sense of their worlds so they can act, in using their propensities for individual thought and socially shared thought and social interaction, perceive order in the social world, and by perceiving that order help produce and reproduce that order, for those orders are constructed of social facts. As W. I. Thomas (1923) stated, drawing on G. H. Mead, what people believe to be real is real in its consequences. Social analysis, however, can make social orders more salient and reflective to participants, influencing how participants act (a process Giddens (1987) calls the double hermeneutic and Merton (1948) calls reflexivity).

\section{MERTON'S SOCIAL STRUCTURE THROUGH INDIVIDUAL CHOICE-MAKING}

The sociology of Robert Merton will be our starting point on this survey of accounts of social order because his sociology finds social order in the 
process by which individuals make choices among alternatives they perceive as socially structured (see Stinchcombe, 1975, for a perceptive analysis of Merton's core themes). That is, the social facts people perceive provide the field upon which they conceive, shape, and choose actions. In so acting they advance their own perception of the socially structured world, reinforcing that vision within the externalized world for others to interpret and respond to. The self-fulfilling prophecy (a phrase coined by Merton) exemplifies this theme starkly (1948).

The relevance of this approach to social order for rhetoric is clear. Rhetorical action creates representations of the social world so to influence the audience's perceptions and consequent actions. Productive rhetoric directly takes this position for the shaping of new discourse while critical rhetoric attempts to recover the position and assumptions of those criticized to uncover their intents, choices, meanings, and actions. The rhetor's perception of relevant genres and their appropriateness to the situation provides structured sets of alternative choices for action, and then the genre choice structures further choices to be made. In making utterances, in essence, rhetors project social orders. In their speech rhetors make visible how they see the world (or at least would like to have others see the world) and attempt to enlist others into that worldview by seeking coordinated responses.

Mertonian sociology is particularly relevant for literate rhetoric. Situation and the related concept of kairos are fundamental to rhetorical analysis, but the perception of situation is particularly problematic for literate communication. Writing and reading enact social situations that are usually not visibly present and offer few immediate, visceral prompts to direct response. Rather, they must rely on their social typifications, including genre, in order to understand, make choices, and act. Thus the situation both in its specific circumstances and its embedding within larger social orders is dependent on the writer's and reader's typified construction of the situation and relevant social arrangements encapsulated in the perception of genre. The literate rhetor constantly recalls and uses social facts to maintain a sense of the situation.

Merton provides an appropriate starting point of our consideration of social order in relation to rhetoric for a further reason, for he integrates the orientation of the individual to a complex picture of social organization. The writer's orientation toward the social group he or she is writing toward is the basis of the writer's stance and the individual specificity of the communication being written. The account I have provided of active, social, communicative selves has drawn on three major traditions that have had great force in shaping sociology: the Marxist, the phenomenological, and the pragmatist. In working with these various traditions Merton invented a precursor to structurationist 
sociology which offers an integration of phenomenological micro-sociological investigations with larger structural accounts of the perceived and reproducing and evolving organization of society (1968b). But to structurationism he adds a particular lesson from Durkheim, in that large social appearances/data can serve as indicators of psychological orientations (1968b, and personal communication). This allows him to harness observation and quantification of large social phenomena to a kind of social psychology, considering how individuals orient towards collectivities and to comment on the larger organizing structures that shape our modern world of action and relations, though with constant awareness of the difficulty of indicators and of differential position, perception, and interpretation (Merton et al., 1979). This complex view allows us to see the individual writer and the writer's intentions within larger conflicted dynamics of social organizations. However, in order to better present this complex social picture, the middle of this chapter will focus on social theory with only a few passing mentions of writing; the last section of the chapter will discuss more explicitly the relevance of this theory to writing.

\section{THE MECHANISMS OF CHOICE MAKING WITHIN OPPORTUNITY STRUCTURES}

Merton developed his view of social structure through a long career of theoretical, historical, and empirical investigations from two vantage pointsconsidering the patterns and mechanisms of perceptions, self-positioning, and choice making of individual agents, and considering the larger enduring historically emergent group structures, which actors perceive as providing the fields and opportunities for action and which influence constraints and outcomes. The first consideration bears some similarity to what we now consider micro-sociology, the detailed observation of individual action in local circumstances, but Merton considers that local actions are conditioned, shaped, and oriented towards larger organizations of society. The second bears some similarity to what we now call macro-sociology, which considers the larger structures of society as ordering lives from the top down, but Merton viewed these structures as emergent by choice making, by the ways people have oriented to the situations as structured and the institutions they have created from those orientations. Thus Merton's work identifies a meso-sociology, a middle range of mechanisms by which the micro occurs with respect to macro and the macro emerges from and is realized in the micro.

Merton, in a widely cited chapter, calls for theory of the middle range (1968b). By this he means theory 
to guide empirical inquiry ... intermediate to general theories of social systems which are too remote from particular classes of social behavior, organization, and change to account for what is observed and to those detailed orderly descriptions of particulars that are not generalized at all. . . . Middle range theories deal with delimited aspects of social phenomena. One speaks of a theory of reference groups, of social mobility, or role-conflict, and of the formation of social norms just as one speaks of a theory of prices, a germ theory of disease, or a kinetic theory of gases. (Merton, 1968b, pp. $39-40)$

These theories of the middle range are potentially compatible with a number of different systems of macro thought, in that they specify concrete mechanisms by which events unfold, but do not necessarily dictate the largest scale picture that can be drawn of society.

This methodological focus on theories that can be grounded in observable phenomena and can be generalized, points toward the kind of concepts that would be researchable and reliably warrantable, and perhaps practically useful. They are theories concerning the mechanisms that link individual local behavior with apparent large patterned organization. Theories of the middle range point toward the mechanisms of the middle range. They are mechanisms in the sense that they show how things regularly happen, organized in patterned ways. As such these mechanisms provide anticipatable pathways for participant orientation, perception, and choice-making. If one has the concepts right, identifying how events, interactions, and relations become organized, one can see them operative in various circumstances, identify choices that can influence events, and anticipate how some events would be likely to unfold. Reflective knowledge of orderliness helps one make choices with a greater power.

\section{TERMS FOR AN AGENTIVE STRUCTURAL SOCIOLOGY}

In elucidating the social field from the point of view of the individual agent needing to make choices, Merton has developed the following key concepts, some drawing on existing sociological work and some his novel invention.

- reference group - the choice of social fields one orients to for value, affiliation, identity, life trajectory (1950a)

- norms and values - the perceived set of behaviors and commitments that are part of affiliation with and participation in reference groups and in 
the performance of roles. These are both what one perceives as part of the fulfillment of that form of life and what one perceives one will be held accountable to in various interactions (1938a, 1973)

- role and role set—specific forms of interaction and interpersonal obligation one enters into as part of one's social positioning (1957b)

- role conflict and ambivalence-the difficulties one enters into because of the multiple roles and relationships as well as multiple statuses and reference groups and incoherence within the values, norms, and perceived behavioral possibilities $(1963,1976)$

- conflict mediation and resolution-as emergent phenomenon, leading to further chosen patterning as favored patterned solutions emerge.

- opportunity structure-the perceived patterned affordances of various status's roles within reference groups as well as obligatory relationship and structures for the fulfillment of needs, desires, enactment of behaviors and goals (1959).

- anomie-the disaffiliation from reference groups that one still remains bound to, the patterned unconventional choices one may make to negotiate the incoherences of values and behavioral opportunities (1938b, 1949a)

- recruitment and socialization-the mechanisms by which individuals are attracted to reference groups and come to learn and behaviorally integrate into the roles, norms, values of a social group.

At a second level he has had a strong interest in the historically emerged patterned structures and institutions which provide the opportunity structures for the affiliation and development of individuals and which provide for the larger social organization of life and the carrying out of social activities and the meeting of needs of society and the individual. At this level key concepts are such things as

- institutions and bureaucracies

- professions and science as socially organized activities

- value and norm systems associated with professions, bureaucracies, sciences, other regularized systems (1938a, 1973)

- patterned behavior and character-behavioral types within institutional or professional space (1940).

- forms of socialization and training that produce professionals of particular dispositions, orientations, and perceptions (1957b)

- differential positioning of individuals within system and with respect to specific needs, perceptions, and opportunities for individual action $(1940,1945,1950 b)$

- socially organized patterns of evaluation and advancement (1968a, 1971, 1995) 
- socially organized events having a tendency or trajectory to particular outcomes (1957a, 1961)

- functions and dysfunctions, manifest and latent (1968b)

- unanticipated consequences as emergent social order or disorder (1936, 1989).

His account of forms of organization as historically emergent from individual choice making over long periods of time has gone hand in hand with his historical studies, beginning with his first book about the relationship between the rise of modern science as a form of patterned activity within the social beliefs, norms, values and patterned economic and political activity of Renaissance and Restoration England (1938a). Considered the founder of the sociology of science, his studies of science have regularly had a deep historical character, all directed toward understanding what has made science a particular field of endeavor that evokes behaviors different than those of other forms of social life, while accomplishing work upon which many domains of modern society have come to rely $(1965,1973)$. He asked similar questions of other modern professions, especially in the health area (Merton et al., 1957) and bureaucracy $(1940,1945)$

\section{MERTON'S RELATION TO STRUCTURATIONIST ACCOUNTS}

Merton's view of structure as constantly produced and reproduced through the actions of agents, through their individual perceptions shaped by prior experience, affiliations and choices, is consistent with later structurationist (see Giddens, 1984) and related accounts (Bourdieu, 1977, 1990), but he provides a more articulated account of the mechanisms by which individuals perceive, orient toward, and make conscious choices within their social worlds.

Structurationist accounts, on the other hand, point more directly to habituated behavior and the dispositions of socialized agents. Typified action is more a matter of habit, affective security, and compulsion than it is of understanding and conscious choice making. Typification, unless it is brought to consciousness for active choice, can be the vehicle of naturalization. Genre and other forms of typified behavior would be chosen and reproduced in most cases automatically, as one would move toward those repeated behaviors that one was most familiar with and one felt most secure within. Giddens, drawing on the interpersonal psychiatrists Erikson and Sullivan, particularly associates repeated forms of social behavior with habits developed within early senses of the secure world, reproducing and extending those secure senses of the world-what he 
calls ontological security (Giddens, 1984, p.125) and holding at bay the more anxiety-provoking circumstances of the unknown or threatening possibilities of the world (Giddens, 1984, $51 \mathrm{ff}$ ). Thus habit is motivatedly reinforced by the anxiety system, and typification is not only a strategic ordering of the world but an affective dynamic that maintains social order through repeated action. More reflexive and intentional strategic orientation toward life is built only on a sense of security that allows one to consider one's circumstances more broadly, so as to restructure one's relations and actions.

Bourdieu has a similar account of deeply seated habitual judgments and actions, bred through early experience. He calls the sum of these habits the habitus or "durable, transposible dispositions, structured structures predisposed to function as structuring structures" (Bourdieu, 1977, p. 72; 1990, p. 53). Bourdieu's views are unclear on the ways in which one's repertoire of perception, judgment, and behavior might be extended either through later sets of experiences within new circumstances or through reflection. Bourdieu at times states that reflective observation of one's habitus may permit some degree of freedom from simply habitual reproduction. At other times he allows for complex and multiple sources of habitus, stemming from many periods in one's life as one enters into new cultural and social fields, introducing variety and change, though not necessarily reflexive choice (Calhoun, 2006).

While reflection on action and reflective action are important categories for Giddens, Bourdieu, and Merton, only Merton provides an extended exposition of the way reflective action is structured. The others consider structured action as pre-reflective, which reflection frees one from. In contrast, Merton's residual category of those things not reflected upon consists of those things unanticipated. Insofar as these unanticipated consequences have functional consequences for the reorganization of social relations they can be seen as the agents of social structural change and the precursors of latent functions, systemically part of the dynamics of social relations but not anticipated or by design, will, or approval of the participants. Most of Merton's social reasoning, nonetheless, respects the perception, planning, choice-making, reflective understanding of circumstances, and reasoned adjustment to situations of participants acting in a world of social facts.

Thus while Giddens and Bourdieu provide deeper appreciation for the role of habit (as do Dewey, 1922, Mead, 1929, and James, 1890) in the compulsive and naturalized patternings of social behavior leading to enduring social orders, they do not provide extended accounts of the reflective choice making available as the individual's reflective perception, judgment, maturity, and emotional security develop so that they are open to more of the possibilities of the world while needing to act in that world. Merton through the idea of reference group 
provides a way to deal with the multiplicity of choices and orientations a person has available in the modern world, and the way the person negotiates among them or finds conflict and ambivalence (Merton, 1976). Habits and habitus can be seen as limit cases of Mertonian social structure where the individual is severely restricted in the orders he or she can perceive and act on in the world, and thus is repetitively and forcefully drawn down repeating paths of behavior. With only a single dominant reference group one is drawn into an affectively powerful set of behaviors, perceptions, and evaluations.

Because Merton's work consistently understands the role of patterned individual perception and choice making, as well as the large perceived patterns of social field upon which one acts, he provides a way to consider how the contribution of recent microsociological work can be integrated into the longer tradition of macro and institutional sociology. Understanding that people make structured choices within fields perceived as structured gives force to such traditional sociological categories as status and roles, institutions, identifiable and structured groups, without turning people into sociological dopes, as Garfinkel (1967) phrased his accusation of macro-sociology. In a Mertonian world individuals are not simply driven to follow norms, nor are they limited to the security of early habits of relations. Rather the adoption of norms, behaviors, and evaluations is part of orienting toward, becoming part of, and participating in chosen social groups that provide perceived opportunity for the satisfaction of desires and needs, that carry out appreciated functions, and that provide a place for one. Larger social forms are not just analysts' categories that impose determinative claims; they emerge out of the practice of individuals attempting to live in a world they need to perceive order in, in order to act. They are, users' categories, in the same way Schutz turned Weber's categories inside out. The theorist and researcher only elucidate what people have made and how they relate to what they have made.

The pragmatic, phenomenological, and Marxist perspectives on human agency that we have looked at in previous chapters all point toward historically emerged social orders and structures and contemporary processes of social organization and ordering. These orders exist only in the enactment, as people orient towards them, respect them, and act as though they existed. They do not exist in some abstract realm of ideal form apart from their practical accomplishment. Certain enduring artifacts may bear markings of social orders perceived at their time of construction, such as architecture or laws, and further these artifacts may facilitate continued enactment of these orders in even more robust ways than before, as when a stadium facilitates and perhaps economically necessitates the production of sports and entertainment events, or laws and court decisions codify practice and provide new penalties for violation. Bureaucracies 
usually embody not only architecture and laws, but budgets, paychecks, forms, files, organizational charts, and a myriad of other material and symbolic artifacts that are integral to the social order. Yet the moment people stop going to the office to do the work or the government loses its legitimacy, or the paychecks stop coming, all those artifacts become empty shells and the bureaucratic social order vanishes. For writing this means texts live only when they are written and read, only when they are in people's minds as part of their activities. The textual artifact at the bottom of a drawer has no social force until it is rediscovered and someone finds it significant and signifying. This is why rhetoric needs theories that tie together the individual and social structures at the point of action and choice making.

\section{THE RELEVANCE OF MESO-PHENOMENA AND THEORIES OF THE MIDDLE RANGE FOR RHETORIC AND WRITING}

Rhetoric is a reflective, agentive, choice-centered perspective on social action. Accordingly rhetoric seeks to articulate strong forms of orderliness to guide people in practice. Any theory that helps specify the social landscape, the roles and relationships authorizing and enacted in rhetorical utterances, also helps enrich our understanding of what constrains, motivates, shapes, and is realized in any socio-rhetorical action. As rhetoricians, we need some such structural theory to give us means to consider the larger forms of organization that we know skillful writers orient toward, and we can't leave it all to local interaction and general tactics. A purely local approach to rhetoric has the paradoxical effect of making rhetoric universal, treating all situations basically alike in character beyond local accidents, and offering the same repertoire of tools and understanding to serve for all circumstances. Only by developing some account of the differentiation of the life worlds that people participate in can we begin to understand how and why forms of writing differ, the dimensions along which they do differ, and the differential means of action in each. Merton's theories of the middle range help elucidate one's position on a rhetorical field and those patterns that can structure rhetorical choice. From this perspective, genres create opportunity structures for action, providing choices and directing energies for the realization of our interests.

This need for a structural theory is particularly important for a writing centered rhetoric, where the writer is typically removed from an audience in time and space, where documents may travel across situations, where print reproduction makes multiplication and dispersal even greater. Electronic technologies now further increase the multiplication and dispersal of times 
and places of contact. In face-to-face rhetoric we can see, hear, and smell the situation and monitor the reactions of the people we are talking with-even in ways that lie below conscious thought. The immediacy, even without reflective tools, may carry us very far in talking appropriately and understanding; yet even under those face-to-face conditions rhetoric offers reflective advantage. In writing situations, our need for rhetoric is all the greater as we may have little immediate situational information and even less immediate visceral feel, so we need to rely on our patterned understanding of how situations go, the organization of social endeavors, the roles and relationships of participants, and the interests and norms of audiences. We need to understand the social systems and actions the texts are part of with all the complexities of affiliation and disaffiliation, conflicting reference groups, multiple sets of norms and attitudes. An understanding of how bureaucracies, epistemic communities work, or institutions and organizations work helps us understand how texts work to carry out the relations and activities with these social configurations.

Further, we gain a deeper understanding of our writing choices if we understand how texts produce and reproduce particular structures through genres' participation in activity system, making some patterns more salient or more dubious, or affect the perceptions of future readers about the social field. Each of our rhetorical acts goes beyond the immediate message it delivers within an ordered social world to continue and modify that order. That continuous enactment or modification of the social order may be indeed as an important consequence of our rhetorical work accomplished by our reading and writing as any specifics of the particular message.

For example, consider the ways our reading and writing in particular genres enlist us in certain identities, roles and relationships (Bazerman, 2000b; Smith $\&$ Schryer, 2008). In filling out a government form we become a client of a social service agency. The writers of the form themselves take on the voice of the institutional inquirer, with legitimized power. If the responder does not comply there will be organized consequences. If the responder does accept the assigned role, he or she then must reveal personal information, and become an acquiescent supplicant dependent on the rules and procedures of the bureaucratic order. The bureaucratic reader of this document produced by an asymmetrical collaboration is in an evaluative role. The reader further becomes institutionally empowered to act on the information and requests presented in the document. The roles, activities, and relationships we may say are brought into being by the documentary matrix (created by long institutional histories). Imagine how difficult it would be to institute an entirely new form of required governmental reporting on, for example, one's healthful and unhealthful behaviors. How many new roles and relationships would be brought into being? 
How much social resistance there might be by people who do not want to be drawn into this new social regulatory matrix?

Scientific articles make claims, but also make the author and text accountable to the critical reading of the profession. As well, the articles contribute to the communal project of advancing specific sorts of knowledge. The writer must be of a certain status to legitimately adopt the role of claim maker and have hope of being published and read, but that role then affords particular kinds of relations of knowledge exchange. Similarly in reading an article, one enters into a complex set of roles, relations and interactions, depending on one's differential position and location in time in space-whether a competitor proposing a similar claim, someone doing related work wanting to borrow arguments and techniques, an historian later seeking to reconstruct the development of ideas and techniques, or a neophyte seeking to learn from the most prominent articles.

In adopting the role-appropriate forms of reading and writing, individuals enact the values and projects of the community or profession by interpreting, selecting, evaluating, and using the meaning of the text in carrying out the valued projects of the field in light of typical assessments made within the field. To write and read as a financial analyst means to value what financial analysts value; even more the act sustains the very activity and value system of financial analysis, keeping the domain alive in the world.

Nonetheless, value ambivalences and role conflicts may arise both in writing and reading because many people may read any document, each with multiple roles and relationships. Because the texts we write are likely to be visible to multiple audiences, we are often caught in role conflicts as writers- how do we manage dealing with different readers who will evaluate the meanings and persona we project from their various perspectives. Writers may be faced with the traditional concerns of embarrassment and betrayal at a revelation, as when the novelist's families and friends see traces of their lives and the attitudes of the author toward them in a roman á clef. Role conflicts, however, may be more a matter of rhetorical complexity, such as when a corporate report writer needs to be persuasive with the managerial part of the audience on the basis of managerial clarity and financial acuity, attractive to the client part of the audience through responsiveness to their needs, and reassuring, supportive, and appreciative to the employee part of the audience. Role conflict theory has useful things to say on how people manage conflict and develop conflict-mediating mechanisms ( Coser, 1966, 1975; Goffman 1959, 1963, 1971; Merton, 1945, 1963, 1976).

In turn the regularly structured conflicts and other interpersonal difficulties engendered by the circulation of texts may give rise to regular structures of communication, interpretation, valuation, and use that help ameliorate or even transform the difficulty into a new set of values, norms and relations. 
For example, the emergent complexity of roles around scientific publication created situations where the same small group of people may be claim makers, critical readers, referees, editors, and claim adopters or rejecters with respect to each others' work. To mediate these role conflicts new norms and values arose since the seventeenth century that changed participants' stances toward difficult situations: an obligation towards criticism, a commitment to a higher goal of communal project of science, a commitment to empirical proof. Appropriate attitudes towards conflict thereby became part of the value system of science. In short, commitment to science trumps, buffers, and reframes the personal insults that are built into the game (Bazerman, 1988).

Written documents often enough become enduring parts of a social systemas a continuing record of past acts, agreements, ideas, and established facts. These records are potentially invocable for new uses and actions. Intertextuality (see Chapter ten) invokes a historical social context accessible to all, influencing the continuing behavior of all. Written laws, court precedent, and court rules all shape ongoing judicial activity. The written journalistic record creates social facts that politicians and government officials must take into account. The documentary records of the health care system structure the behavior, information gathering, and judgment-making of health care providers as well as the opportunities for interaction and service for clients. Utterances are acts that condition the landscape for all future actions, but written documents particularly stay more visibly and enduringly on the landscape, may travel further through time and space, have greater stability, and may be multiply reproduced (Bazerman, 1997). For such reasons written texts frequently attain special legal or epistemic status and may gain higher degrees of social attention.

Merton's sociology points to the statuses and roles one holds with respect to the audience, the specific roles one is enacting in the utterance, and how the multiplicity of roles and relationships with parts of the audience may create conflicts. Rhetoric needs to have as much a sense of the disaffiliations and anomie that may condition an audience's response as the forms of identification and subsumption. Mertonian sociology also points to the ways in which the relation with the audience provides an opportunity structure for certain kinds of needs, interests, and actions that can be realized through communication. It additionally points to the structured advantages certain rhetors accumulate and the relative disadvantage others are put at, which Merton labeled the Matthew Effect (Merton, 1968a, 1995).

Finally, if writing mediates social processes, learning to write is a process of socialization into the practices, relations, positions, and activities of social collectivities. Writing in any domain is more than a matter of gaining technical mastery, although that technical mastery may be an important part of becoming 
a successful participant in the group. We would do well to start looking at writing in relation to socialization theory and socialization mechanisms. There are already a few forms of educational theory that work on a socialization model, particularly those concerned with an apprenticeship model, such as Legitimate Peripheral Participation (Lave \& Wenger, 1991; Wenger, 1998) which is concerned with integration into communities of practice, and other theories that are concerned with the formation of learning communities, but all these could be aided by a more detailed account of how individuals are recruited and socialized into groups and adopt groups as reference for behavior. Writing itself provides the means of creating social presence in literacy-mediated social groups; this situation suggests that we need further writing-specific accounts of socialization. For all these reasons rhetoric can use theories which can articulate complex role sets, complex activities, differential social positioning and goals, role conflicts and conflict mediating mechanisms, unanticipated consequences and other emergent social phenomena as they pertain to writing. 



\section{CHAPTER 7}

\section{FROM THE INTERACTION ORDER TO SHARED MEANINGS}

One lesson of structurationalism is that social order does not exist in an abstract space above and beyond the actual sites of social relations, but rather must be constantly remade and maintained in lived spaces of interaction. Accordingly, any larger patterns of social order and organization that may exist must be constituted and built on patterns and relations played out at the concrete level of individuals in individual events. This recognition extends beyond simply seeing evidence of social orders in concrete data, as the consequences or ramifications of more abstract orders. Rather structuration directs us to look at the interactions as themselves the site at which order is constituted.

The grounding of society in concrete interaction suggests that social order can be effectively studied in concrete individual interactions. The advent of recording technologies has facilitated researchers in capturing interactional data, examine them, slow them down, and analyze their social realities in great detail. Conversational analysts study social orders through microanalysis of synchronous talk interaction, either face to face or telephonically (Lerner, 1993; Schegloff, 1987). Yet writing facilitates and connects people, events, and interactions across time and space, creating objects for co-orientation, co-relation, and action that do not rely on co-presence. Further, the typifications, patterns, and social organization of communication that facilitate communication at a distance foster and structure larger social and organizational aggregates. The textual, symbolic, and concrete objects that are multiplied and travel across time and space, furthermore, provide a concrete means of understanding how social order at a distance is possible; further, the study of how people produce, engage with and use these objects can open up some of the fundamental mechanisms of larger social orders. Nonetheless, the project of grounding social realities in the concrete interaction is a powerful one that provides us guidance in pursuing larger "at a distance" orders in concrete ways. Therefore, before we consider how interactional order is enacted in the literate world, we should first consider how people studying face-to-face interaction have pursued the project of understanding the interactional order. 


\section{THE INTERACTION ORDER}

There is no more powerful and fundamental investigator of the interactional order than Erving Goffman. Fortuitously for our purposes, he has also considered communication in somewhat less personal circumstances, such as in lectures, over radio, and even in print, providing bridges to the literate interactions that are the focus of this volume. So it is with a discussion of Erving Goffman that we begin this chapter, and particularly with his essay "The Interaction Order" (Goffman, 1983). In many ways this posthumously published essay is the culminating theoretical statement of his career, framed as the 1982 presidential address for the American Sociological society, but never delivered in person as he was battling cancer.

In this essay Goffman starts from the premise that we spend much of our life in the presence of others and that the conditions and needs of life ensure this. For us to cohabit this shared space successfully (that is to meet our individual and shared needs without undo conflict) we need to make plausible and appropriate surmises about each other's status and relationships, but even more about intentions and goals. We get much information for coordination visually from observing each other's actions, orientations, gaze, and appearance, including both ritual and spontaneous elements. Speech greatly facilitates and makes more efficient these coordinations. Further, this information is gathered and used within the concrete situation perceived by the individual. As Goffman notes, "It is social situations that provide the theater in which all bodily displays are enacted and in which all bodily displays are read. Thus the warrant for employing the social situation as the basic working unit in the study of the interaction order" (1983, p.4). We may equally say it is within situations that speech is heard and interpreted.

Significantly, Goffman's fundamental attention to the social situation mirrors rhetoric's fundamental concern for the rhetorical situation or kairos. It is through the recognition and construction of situations that people find order in interaction, so as to be able to anticipate that actions will be effective. To do this they must have a way of perceiving the specifics of the immediate situation in the here and now as it unfolds and of associating that with what they perceive as repeated patterns of events. These perceivable patterns need to be shared with other co-participants to the degree that their understandings will coordinate or align in producing interactions that can unfold in ways that make sense to all participants. That is, if they do not have sufficient alignment in understanding the event, conflicting definitions will produce behaviors that others will not be able to make sense of or perceive as cooperative, putting the event in danger of disintegrating. Schutz might call these shared patterns of perceptions typifications, while Goffman calls them cognitive presuppositions. 
To use the metaphor drawn from literacy that Goffman himself invokes, the situation and people's behavior must be read (1983, p. 4) and, therefore, must be readable. To use a less literate imagery, one must be able to make sense of one's immediate surroundings and the behavior of others in a way that is sense-able, that is, accessible to human sense-making procedures. Some microsociologists indeed argue, people need only that immediate sense-making to operate within the world, and that the meso-and macro-social structures are for the most part constructs of analysts and not the real world in action. Goffman actually holds a contrary position, saying that larger social structures have an independent influence on our lives, though they may bear on the micro and the micro might bear on them. He cites the example of being informed by an employer or a spouse that your services are no longer needed. Although the particular form of the sharing of this news may have some short term emotional consequences, the fact is that a day or a week or a month later, the change in business or personal arrangements will far outweigh the amicability of the termination interview. Nor, as he points out, do amicable interactions seriously change the underlying inequities of class, race, or gender.

Nonetheless, the concrete mechanisms and consequences of these larger social arrangements must play out and be delivered in a sequence of real settings, as sites for local action. These patterns, typifications, or cognitive assumptions are operative in a number of ways: through the belief and orientations that focus perceptions of situations calling for action; in the means and resources available to be deployed in the situations; within the artifacts and arrangements which provide the grounds of local interaction; and in the significations deployed in the moment-by-moment improvisation of behavior within the situation. Social life and the enactment of meaning exist only as they concretely happen during evanescent wisps of unfolding moments as perceived by the participants. Yet these vanishing moments leave a residue of enduring artifacts, texts, arrangements, and habits that create a complex mutable order that gives some shape and predictability to future moments, which are themselves equally concrete and evanescent, saturated by semi-stable, attributable meanings. While artifacts and memories may travel across situations, yet they exist in people's life world in the evanescent here and now formed by attention, meaning-in-action, and interaction. While there may be some aspects of human existence that may be understood to a significant degree without reference to the unfolding moment (such as the structure of organic chemicals found in the body-but even bodily chemical states are responsive to our neurological attunement to situations), almost all the questions about language and writing (once you get beyond the chemistry of paper and ink), depend on meanings given and taken by people in the moment. So rhetorical force is directly and irremediably enacted at the 
interpreted moment, no matter how much textual artifacts may endure across multiple situations and circumstances to provide a commonality of situations and conditions. Nor does even a fixed text mean the same thing in all situations and to all participants; the physical existence of documents just results in the document being available for inspection or other use by multiple participants in multiple situations (including legal evidentiary, analytic, and academic situations). In each new situation the meaning of the text is reenacted within the habits, practices, interests, and arrangements available to the participants.

Goffman identifies two reasons for our being attentive and compliant to the interaction order-that is, the set of understandings that allow us to cooperatively create situations within which our behaviors make sense to others in ways that align with the sense we wish them to make. One (which Goffman calls the "social contract" reason) is that we have much to gain by respecting this order at small cost and much to lose if the interaction order dissolves. That is, by recognizing and framing our behaviors within the order, we are able to act with others, and if we do not attend to the order we would lose that ability to act with others and would gain nothing. As Goffman points out, even criminals and others who normally violate the norms of the interaction order, rely on those norms to locate their targeted violations and to hide their misdeeds from easy notice. The second reason (which Goffman calls the "social consensus") is the unthinking assumption that what one sees around one is how people act and there are no plausible or sensible alternatives - this is similar to what the phenomenologists would call "the natural attitude." The social contract and the social consensus both lead to the conclusion that the constraints that apply to oneself also apply to others and that one should submit to them (except for conscious and focused violations, such as by criminals).

\section{PROXIMATE INTERACTIONAL ORDERS AND DISTANT}

Goffman's focuses on the immediate, proximate space with its temporally unfolding events visible to the participants, even as what is attentionally relevant may expand or contract as events unfold and as definitions of the situation change through shifting frames attributed to the visible, audible space and towards which the participants align. This shared alignment defining the situation, Goffman calls footing. His well-known essay on "Footing" (1981) and his volume Frame analysis (1974) elaborate these ideas most explicitly.

This proximate face-to-face space creates an urgency, because we are visible to others and open to their evaluation. If we are not responsive to the interactional order, others may project their interpretations and reactions onto the space. If 
we violate the presuppositions or typifications or frames active for others in that space, or if we do not discipline our behaviors to be readable by others, we may be hailed to attention, rebuked for inattention, accused of failing to respect our responsibilities to the moral order, or even cast out as irrelevant, irresponsible, or insane. Goffman by the end of his career even placed this aligning to the interpretable public order of those immediately around us as driven by the desire not to be deemed insane. This position resonates, with a punitive clarity, with Adam Smith's understanding of moral sentiments arising out of our seeing ourselves as others might see us and G. H. Mead's view of our forming our sense of ourselves through the eyes of others so that we can make ourselves understood by them.

Yet while Goffman makes a strong contrast between immediate social spaces of the interactional order (defined by mutual visibility imposing mutual readability) and structural social order (where we must be responsive to forces and people not within our immediate sphere of mutual visibility and mutual real time readability), he himself examines some interactions that had more tenuous holds on full immediate reciprocality — such as scripted lectures (where audience responsiveness and attentiveness may influence delivery, but rarely disrupts the flow of talk) or radio addresses (where people's attentiveness and reactions are invisible - even to the extent as to whether any listeners are tuned in) (Goffman, 1981). In these cases Goffman looks at the speaker's or author's anticipation of the audience's interpretive frames, and the author's attempt to shape, mold, and invoke those interpretive frames and footings. Accordingly, while Goffman's typical sites of investigation-people managing pedestrian traffic on a crowded sidewalk, maintaining face in a business meeting, or managing roles in a psychiatric ward — may be viewed as being on one end of a spectrum of immediate visibility and moral accountability, they are not divorced from other points on the spectrum where our financial life is shaped in our interaction with institutional statements, monthly payments, and readings of our bank balances; or our citizenship life is framed around periodic encounters with ballot boxes; or our intellectual life is formed by our reactions to the words of authors within the journals we read. All of these interactional spaces must be readable and read, and our presence depends on our participation, stances, alignments, and frames.

\section{FRAGILITY OF WRITTEN INTERACTION}

In writing, however, the problems of attentiveness and alignment are far greater than in face-to-face interaction. Without full, embodied co-presence 
the channels of communication are more limited, the opportunity for noting response and making adjustments to retain attention and alignment are rarer, and the compulsion for attention and accountable response is more tenuous. The largest issue is that many people do not even look at or read (in the narrow sense) another text, even when there might be some expectation they do. Even personally addressed letters go unread, let alone group memos. Books that "all citizens should read" may sell a few thousand copies, with many purchasers never opening it or putting it back on the shelf after a few pages; only a few may read it cover to cover.

In written communication, rarely does a text press itself on us demanding attention, unless it touches an inward compulsion. Of course there is the letter from the Internal Revenue Service or bank or other powerful social institutions that a person dare not ignore. These cases of high compulsion and accountability identify strong interpretive frames that demand attention and limit the likely actions. The letter from the IRS is likely to have only a few kinds of gists-requesting further information, commanding further payment, calling for an audit, presenting a refund. These gists correspond to form letters and narrowly framed genres. Individualized messages from the IRS are contexted within regulations, past communications, and personal finances that locate the meanings, actions, and urgency of attention. Even then there are some people who throw away such notices unread, claiming they will have nothing to do with the IRS, until the IRS sends out the police to take physical possession of the people or their assets- thereby compelling attention.

More often in reading, though, texts are self-selected. Even at the office, which files we deem relevant and then examine are a matter of judgment. Unless we decide to go to a file, or pick up the morning newspaper, or click the link to a website, there is no interaction. The text remains unreadable in the interpretive sense because it is unread in the decoding sense. Attention is not just a random matter, for what constitute our interests or what strikes us attractive or meaningful, depends on the sense of meaning we are building about our lives and the world. That sense of meaning of our life world includes evaluations of the kinds of meanings we believe various kinds of documents will contain for us: "Oh, I never read magazines like that, because they don't have substance ..." or "I used to read it, but then I grew up," or "that stuff is too hard to understand," or "it may serve the interests of managers but not consumers."

Even after readers pick up a text, attention may wander, and interest may fade. Many documents are fallen asleep over, skimmed, put down. In short, readers escape or diminish the text's presence and withdraw from the interaction before the relationship, the cognitive attention, the effort to create shared meaning goes very far. It is as though people walk away from you as you 
start speaking, or turn away to look at the TV, or demand you jump ahead to the point. Even in situations of structured accountability, students do not get through the assigned reading, managers do not read the reports in any detail, users don't follow the instruction manual, and applicants don't attend to the regulations for submission.

Even if people read a document all the way through, they read at varying speeds with varying levels of attention and retention, from the perspective of their own understanding and goals. Variation in reading becomes visible in those unusual circumstances when people actually compare their readings, as in classes devoted to discussing specific texts, whether of poetry, philosophy, or social theory. Under such circumstances differences in what people take to be the meaning are likely to emerge along with disagreements as to what seems most important or salient to each reader. Advanced training in specific disciplines of reading, whether literary, theological, legal, or philosophical, may serve to proliferate alternative readings, even as training excludes certain naïve or inattentive ones. No matter how well crafted a text may be it is always porous, even in the law-that is why we have lawyers and courts. This is the puzzle the hermeneutic circle tripped over (De Man, 1983; Gadamer, 1975; Shklar, 2004), that reader response theory (Fish, 1980; Iser, 1980) attempted to account for, and new criticism attempted to ameliorate through close reading (Richards, 1924, 1929), even though new criticism quickly became a means to proliferate even more readings (Brooks, 1947; Empson, 1947).

\section{THE INVISIBILITY OF FRAGILITY}

The fragility of face-to-face communication is often hard to detect because participants regularly adjust to each other to carry situations forward, and repair when minor breaches appear to occur (H. Sacks, 1995). Often our interlocutors anticipate breaches and adjust for them, even when we do not perceive any threat of rupture; we call such behavior apologetic, accommodating, or anxious. We work hard to hold situations together and maintain at least the appearance of mutuality, as the ethnomethodologists noted by identifying "let it pass" as one of the primary methods people follow in attempting to make sense of each other and situations (Garfinkel 1967, p. 3).

We notice the fragility, however, when situations fall apart, hard feelings ensue, and people create unpleasant characterizations of former interactants and the behavior which violated expectations. Garfinkel's notorious breaching experiments revealed how even small deviations from normatively expected behavior can lead to very large social ruptures (Garfinkel, 1967). Such 
experiments test the limits of expected behavior and reveal the depth of moral importance we place on others holding up their part. Such experiments also reveal the pressures on us to follow expected behavior.

The fragility of literate interaction is even more invisible, because the rupture happens out of sight. People rarely let us know if they have not read what we wrote, if they lost interest, or were so outraged they stopped reading. Nor, even if they finish, do they report back to us the meaning they got from the text. We happily go along believing they read what we wrote. Ask any author who is lucky enough to be widely reviewed or discussed in other publications about how well their readers understand their work or even if their readings seem at all plausible, and you may see another side. On the other hand, it is rarely in the author's interest to contest the readings, for at least the text is being read and discussed. The common wisdom of authors is to let the text speak for itself. For the most part people hold their reading privately within themselves as part of their own amusement, intellectual development, curiosity, formation of beliefs, or accumulation of information for action. If they compare readings, their comments may be sweeping or vague, so that rarely is anyone likely to contest in detail what they gleaned from texts.

Only in limited cases is there in fact any exigency for us to come to shared readings of any text in any detail. Immediate operational needs can necessitate shared interpretation, such as a group making sense of a manual to carry out a repair, but the readings match only to the level needed for immediate practical purposes, which then gets taken over by the exigencies and materiality of the action and artifacts themselves. Embedding reading practices in complex sets of shared social practices may also help align readings. Although students first encountering a chemistry textbook may have all kinds of unusual understandings of the text, if they solve enough problems, do enough experiments, discuss enough phenomena, and engage in enough other professionalizing activities over years, their readings of chemical texts will align with the readings of those who have become their colleagues. Specialized practices of asserting understandings of readings before commenting, such as associated with Rogerian argument (Rogers, 1961), or the review of relevant literature in scientific work are attempts to create shared communal alignment to prior texts to then carry forward discussion. When people have significant stakes in comparing readings in detail, professionalized forums and disciplined technical practices may arise and may be honed in interpretive debates—such as in law, philosophy, literary studies and theology. Sometimes in these forums the discussion leads to people to consent to more aligned readings, as Fleck (1979) in his observations of thought collectives, and Fish (1980) in his interpretive communities. Yet even in professional forums no exigency may press for resolution, with people simply 
refining and arguing for the validity of their particular readings. Only when there is a judge or jury to determine the authoritative reading does that settle the question, but even then usually under duress and with muttering of those who feel they have to buckle under to the state.

When texts fail to create reasonably congruent meanings adequate for cooperative practical purposes we have many ways of accounting for the breakdowns, hiding the fragility. Easiest and most common is blaming either participant. Either the writer can't write or the reader can't read. Other kinds of stigmatizations and consequent hostile elaborations can hide the breakdown such as accusations that the other person lacks understanding of the issues or is misguided philosophically or is cynically driven by ulterior motives. This is not to say that such characterizations are not sometimes warranted, nor to suggest that critical reading or rhetorical savvy are bad things. Yet these characterizations can be mobilized in instances of communicative breakdown.

Such characterizations masking the breakdown of literate relations are made more tempting because of the semi-privacy within which we usually carry out literate activities - just us and the book or computer terminal. In mental semi-privacy we can tell ourselves stories that remove us from the challenge or difference of the text we are reading or from the difference of those who might read our text. The fact that education and reading are so surrounded by a hortatory ideology of opening up the mind, entertaining difference, and learning the other side, suggests just how difficult and exceptional it is to address texts that do not match comfortably with our preconceptions. On the other hand, the common experience of becoming more sympathetic and understanding of a writer once you hear them read or talk in person suggests just how much the isolation of literacy limits our alignment to others' words and stances (See Inglese, 2010 for a study of how showing video interviews of famous writers to students improves the students' understanding and sympathy for those writers' texts). This value for seeing the writer as a person is matched on the writer's side by the well-known importance (and difficulty of obtaining) a sense of how readers actually respond to what the writer has written. Yet writers often resist accepting any but the most laudatory response from the readers. Even experienced authors must struggle to receive comments with equanimity and to evaluate them evenhandedly.

Characterizations of faulty readers and writers usually assume that an ideal text-well written, carefully read by competent writers and readers-should carry all the burden of successful communication. We tend not to think of the text as a fragile mediator in a complex system within interpersonal human space, and that breakdowns might occur or ramify anywhere. Certainly attending more intently at the mediating artifact with skilled tools of interpretation is 
useful, because texts are the scene of transfer of action. Yet the text only sits in the middle of a process, no matter how well and skillfully the text is attended to. So we must view the processes of sense-making within social configurations, rather than taking the text as a universal conveyor of meaning, accessible to all in any circumstance.

\section{CREATING ALIGNMENT AND READABILITY IN WRITING}

The fragility of written language puts great pressures on writing to be understood as situationally relevant to the reader, worth attention, readable, interpretable, and useful for the readers' purposes-all within the context of a limited asynchronous communication channel of words (and graphics or other enhancements) on paper or a screen. Despite difficulties, a successful text must evoke in the mind of the reader meanings congruent enough to the intentions of the writer and supportive of the desired actions to be taken by the reader so as to complete a satisfactory transaction. While the worlds of meaning evoked in the reader by literary texts are sometimes considered in literary theory, worlds of meaning are in fact pervasive in all literate interactions and not easy to accomplish. They require high degrees of work by both reader and writer, cooperative stances between them, and a willingness to discipline selves to the technicalities of inscribed language, including the most basic tools of written language such as forms of handwriting and inscription, orthographies, grammars, and punctuation conventions, to be discussed in the next chapter.

One of the key mechanisms of attaining alignment is to cast messages in familiar terms and typified forms. The need for intelligibility thereby reinforces reliance on genres. If, for example, you need comparable specific information from a group of respondents, you are likely to use questionnaires with questions in familiar formats, so respondents know what you are asking for and how they might respond if they so choose. The more unusual the information you seek and the more open-ended or unusual the format, the less reliably people will know what to answer, and the more difficult their responses will be to interpret and compare-and the lower response rate you are likely to get.

Other devices for locating and aligning participants are narrative reconstruction of the situation of writing or of likely reading use, reminding readers of shared information, and explicitly identifying relevant shared intertexts. Familiar designs, appropriate publication venues, familiar phrasing and a narrowly defined technical vocabulary, or other presentational variables 
can also help readers identify and align with the meanings projected in the text and tap into the representations they already have at hand.

Formulations that rely on familiar community beliefs for their coherence is what Aristotle referred to as enthymemes, used as a persuasive device. If a speech doesn't make explicit all assumptions and logic, but relies on the listeners to make the connections and provide the facilitating beliefs, the listeners will evoke feelings and meanings already in their mind and which they feel are their own. They will also find the speaker to be of a like mind and therefore to be trustworthy. Further, insofar as they must think actively to gain the meaning, using what they already know, conclusions become their own, for they have thought it through. Thus the entire shared performance is likely to create a common bond between speaker and listener. In writing, this sense of common meaning and reasoning is even more important to maintain sense of situation, attention, and meaning. But if enthymemes and familiar genres define the total domain of meaning aroused, then one never brings the reader beyond the familiar, as in the tiring diatribes of partisan journalism or the repetitive celebrity "news" varying only in the names and locations.

\section{THE INTERACTIONAL POTENTIAL AND CHALLENGES OF EVOKING NOVEL MEANINGS}

On the other hand, writing creates opportunities for more elaborate individuation of opinion, extended originality of statements, and more finely honed articulation. The reflective, extended process of writing can remove the writer even further from the reader and the likely contents of the reader's mind. This puts a high burden of mutuality and hard work on both reader and writer to create meaning across the thin stream of inscribed words. This mutual hard work starts at the level of reference, to ensure both interlocutors identify closely enough the objects in the world and concepts evoked by the words to go down sufficiently similar thought paths. Even terms for common objects, such as chair, have a range of mental associations, each of us picturing a prototypical version of each (whether an upholstered easy chair or a fold-up metal utility chair) and having a range of easily imagined variations (some would readily include a natural rock formation and others a multi-seated bench as chair, while others might have to think a bit to understand these variants as chairs). Pronouns and other deictic terms typically cause problems for less experienced writers because they are not as skilled in directing readers to the thing they want to indicate. Further, what is readily attended to and accepted as part of the scene includes 
a cultural deictics of attention and boundaries. H. Sacks' (1995) analysis of membership category devices, Hanks' (1990) analysis of cultural deixis, and Bakhtin's (1981) consideration of chronotope all elucidate the cultural and genre horizon of expectations about what a scene is likely to include.

The problem with alignment of concepts is even greater than of material objects. The exact class of events covered by a concept, how a concept operates in relation to other concepts, what system of reasoning the concepts are related to, personal idiosyncratic use of terms within private cognitive worlds, and similar concerns present problems in alignment of imagined meanings by writer and various readers. Disciplinary training attempts to alleviate some of these problems, by long enculturation into disciplinary knowledge and practices that restrict ranges of meanings; yet even within disciplinary discussions theoretic disagreements, misunderstandings, and other misalignments create slippage in conceptual meanings. Particularly as people are trying to articulate novel concepts they are likely use key terms in ways that may not always appear fully coherent to peers as they reach towards new frameworks of perception.

The problem of alignment in meaning-making goes far beyond the identification of individual concrete or conceptual terms, as texts create large networks of meanings that must be understood within the structure of the text and in relation to other meaning structures that might be brought to bear to understand and evaluate the text. How each claim, each sentence is related to each other, what larger structures of meaning emerge from texts, and how that meaning fits with other existing frames of thought present problems for both readers and writers. This problem appears at ever more sophisticated levels as readers and writers become more skilled and engage in more specialized domains with more subtle distinctions and reasoning, drawing together larger complexes of ideas and evidence. Even though the text may unfold temporally in a sequence of sentences, the meaning emerges only as the reader keeps the whole meaning structure in mind simultaneously. Similarly, the meanings evoked when referring to prior texts can be problematic. Readers may find different issues salient in each prior text cited, interpreting them differently, assigning different evaluations, and relating them differently to each other. Even keeping track of who holds what opinion in an article that cites multiple people is difficult, let alone what position the writer holds with respect to all the texts discussed and the overall topic under discussion.

Genres and other typifications can serve to align and limit interpretation, but the more typified and common, the more they restrict the potential meanings that can be made. Genres may even have the perverse effect of limiting the precision of message, as there are standards of approximation good enough for 
typical purposes built into genres. Thus if a genre typically has only broad nonquoted references to sources it encourages a belief that the source texts are clear and univocal in their meaning and only the most familiar meaning is to be drawn from them. Similarly, the use of standard sectioning of an argument decreases the burden on providing an explicit rationale for the continuity of the parts and the architectonics of the whole. For this reason we often find a paradoxical consequence that the most typical articles (the ones that are closest to conventional expectations), although the most easily read, may not be the most influential, because they bring little novelty to the discussion. Sometimes highly influential texts within disciplinary or professional contexts are hybrid, bringing unexpected resources and modes of representations to the communal reasoning. These hybrid contributions cannot abandon or ignore disciplinary expectations, but they bring in and integrate other recognizable modes of discussion to supplement the conventional meanings. These hybrid supplementations may be controversial and some may view them as hard to understand, inappropriate or irrelevant, but others may see the necessity for the new meanings. Such controversy and simultaneous expansion of reasoning occurred in the United States Supreme Court in the case of Brown vs. Board of Education of Topeka where social scientific evidence of children's selfconceptions was brought in to argue that legal principle of "separate but equal" was faulted because it led to unequal consequences.

The complexity of novel meaning can create cognitive and emotional strain on both writer and reader. Writers find it hard to think in the new ways their arguments demand of them, sometimes not sure of where their ideas are headed, because their own prior beliefs and knowledge no longer provide firm guides. Further, they may be appropriately anxious that others will not follow them to their new meanings or will reject them for writing such strange and heterodox things. The reader as well needs to struggle against preconceptions to follow new meanings without rejecting them out of hand as being unclear or outlandish. Often enough I have heard people complain of the difficulty of texts and claiming the texts are poorly written when by all obvious textual measures of vocabulary, sentence complexity, cohesive markers, or paragraph and text organization the texts are not in any way exceptional. But the meaning was unusual, introducing unfamiliar material, putting familiar material in unfamiliar perspectives, or looking at issues in greater detail than usual. These problems of articulating and understanding unfamiliar meaning can occur at any level: when a high school student must write an essay that goes beyond plot summary and a teacher must help the student identify the nascent thought being born or when a Wittgenstein is trying to articulate a new philosophical perspective and readers are trying to absorb it. The problem remains the same 
of how writers and readers can align well enough over a text for adequately congruent meanings to be evoked.

An interactional perspective helps us understand more deeply how creating congruent constructs of the communicative situation are essential for aligned participation and meaning-making, yet how difficult creating congruence is in ways that go far beyond technical skills of inscription, orthography, and grammar. While face-to-face talk affords many devices to hold the interaction together despite transient misalignments and threatened ruptures, literate interactions at a distance have only attention to the written word, in production and reception, as a mediating mechanism. Literate meaning-making attention, carried out in the imaginations of the separated participants, is fragile, pushing participants to engage in the most normative activities and meanings in order to increase the chance for robust alignment of understanding. Yet the potential of writing to create novel meaning tempts the writer to be more ambitious and challenging in what the text attempts to convey. Successfully conveying substantially novel meaning requires both writer and reader to attend carefully to the nuance and architectonics of the text. Even with high commitment and skill on both sides, the level of co-alignment and mutual understanding is often much less than the fixedness of the inscribed text might suggest. Substantially novel texts, if they convey fresh meanings perceived of potential value to the readers, reveal their success in evoking extensive discussion among readers as to the meaning. The meaning is not fully obvious and univocal from a plain reading of the text. The complexity of constructing an effective interactional order helps us understand that the aim of writing is not a "perfect text" but maximum alignment of meaning construction between writer and reader, creating meanings for the reader in a way that is congruent to the meanings the writer desires to evoke and that lead to the desired consequent thoughts and actions that the writer hopes for. 


\section{CHAPTER 8}

\section{LINGUISTIC ORDERS}

The socially and psychologically complex interaction between writer and reader is, however, carried out through the thin line of words transmitted on the page. From a writer's point of view the task, is, as Hemingway famously said, "getting the words right" (Hemingway, 1958). Words are the material we work with, what we inscribe to create our meanings and influence the readers. When we are done writing, they are what remain on the page for others to see.

Multimedia technologies, of course, do now extend the resources which can be mobilized on the page, but still language remains central to the craft of writing. While some of the same issues of communication at a distance discussed here may be applicable to them and their integration with the written word also pose important issues, I will not attempt to subsume these other communicative arts into principles developed for writing, and I leave the analysis and theory of multimodal representation to others.

Since words are essential to the craft of writing, it should be no surprise that the disciplines of language, written signs, language order, and language manipulation have been central to writing practice and pedagogy. Grammars, handbooks, dictionaries, thesauri, books of sentence and genre models, vocabulary builders, and exercise books have emerged in the last few centuries as the practice of writing spread and became organized into larger systems of influence through schooling and printing. Language reference books have become the companions of writers and editors. Reference books create a common coin of mutual understanding and easy interchange, disciplining the idiosyncrasy of each of our language choices and expanding the repertoire of communicative tools and expressive potential. It is not an accident that young writers become fascinated by books of phrases and figures, stylebooks, grammars, and dictionaries of their own and other languages. Nor is it surprising that parodies highlight the lexicon and structure of different genres and styles. Nonetheless, these reminders and regulators of the linguistic order often evoke deep ambivalence in writers. Regularity and commonality may seem the enemies of creativity, meaning, and authenticity. They remind us of the conventional against which we create the particular, unique, and urgent within our texts. Attention to the tools rather than the message seems to detract from the communicative impulse. Resorting to the familiar invites cursory reading and rapid categorization rather than immersive engagement. Further, the regulated orders and disciplines of language necessary for mutual understanding suggest 
social histories of class, power, hierarchies, orthodoxies, and other forces that favor "proper" language and restrict speaking rights to those already privileged. Stigmatizing the language of others as disorderly and improper provides a ready way to discount discomforting meanings, affiliations, and actions.

This tension between order and novelty is necessary and productive for writing as we must struggle with our tools to construct the words that will capture our meaning impulses and open the minds of our readers to those feelings, ideas, and actions we wish to evoke. We must work with a medium others understand, but we must evoke a freshness of attention to make meaning come alive to activate the spirit at rest. If the reader is already in action we must then speak the common language with an uncommon relevance. Such tensions excite linguistic creativity to push the boundaries of the sayable, ever inventing fresh tricks to use what we have in new ways, to propose new language by analogy and metaphor, to borrow and transfer from one domain to another. Human cleverness and responsiveness to situations push language to its limits. Insofar as we articulate orders to facilitate and regulate language use, others will use that order for reflective creativity, using the very terms of order to violate and transcend. The very articulation of an order creates a new abstracted position from which to play and innovate.

\section{THE IMPORTANCE OF THE ORDERLINESS OF LANGUAGE}

It is, paradoxically, both impossible and easy to overstate the importance of the orderliness of language in the emergence of modern forms of human life. Language is entwined with almost all we do and how we think about what we do. Written language has then entwined those actions and thoughts into larger enduring sets of representations and meanings spread across broader and more distant groups of peoples. Language and its progeny writing provide the means to construct cooperations, meanings, knowledge, and the interactional space to enculturate youth into the content and practices of interaction. Thus the orders of language order human relations, belief, and knowledge while focusing the processes and practices by which we commune with each other. The orders of language can be seen as infrastructural to human community and consciousness and, therefore, important to understand for strategic reflective choice making for enlisting cooperation, creating knowledge, and refining thought. How then can we overstate the importance of how the orders of language pervade human life?

Yet, this importance may delude us to believing that language contains all thought, experience, meaning and knowledge of the world - that all is to be 
found in language. We are tempted by the search for universally wise texts and Borgesian libraries that inscribe all of knowledge and will answer all questions we may have and all questions yet to be asked. Indeed, within fundamentalist communities, belief in the universality and infallibility of one or another sacred scripture precondition stances towards the textual world of secular knowledge. Nonetheless, language is not all of life and does not preexist life. Sophisticated non-human biological and social creatures without language have experiences, cooperate, and share attention and orientation toward their environments (see for example, Johnson \& Karin-D’Arcy's 2006 and Tomasello's 2006 reviews of non-human co-orientation). Further, we attend to, consciously respond to, and even mutually co-orient to many aspects of our experience without attempting to express them in language or inscribe them in our books. Even less linguistically articulated are those aspects of our experience we react to unconsciously. And even those things humans cast into language only get their meaning if people engaged in action attend to and make sense of the linguistic representation.

Spoken language is nothing in itself except disturbed air and written language is nothing except dark pigment on wood pulp or electrons on a display. Those traces would not be there unless people intentionally created them and invested them with meaning. In this respect whenever we consider language and its orders, written or spoken, as autonomous and meaningful in themselves without considering how those orders are understood, developed, and used in practice by human beings in situations, we are overstating the force of language orders. We can overstate the importance of language if we claim it as absolute, autonomous, and determinative.

In another way, it is very easy to understate the importance of the orderliness of language and written language, not noticing how infrastructural they are for all we do. Language and its orders are so pervasive they become invisible, lost within the activities themselves. We think thoughts without wondering about the language that expresses the thought, let alone about how the particulars of our language and its ordering principles prompt, constrain, and focus thoughts and actions. We think about our knowledge without questioning the material out of which it is made as the amateur appreciator of sculpture might not notice or think about the stone, its properties and the chisel marks. We are so engaged in the actions enabled by language we may not even notice the way language shapes forms and guides those actions. Though lawyers and economists spend much of their days processing and producing texts, they will likely say they are arguing the law or making economic projections rather than reading or writing. Nor are they likely to reflect the way the formulas of their language create the means of making the expressible thoughts of their field. Likewise, in our 
everyday activities, all of us will likely say we are shopping rather than writing shopping lists, reading packages and labels, and mentally calculating costs.

Yet again, it is difficult in another everyday way to forget prescriptive orders of language, as we are constantly being held to norms of language. As children we are instructed and corrected by teachers, parents, and other adults. As adults we are constantly held accountable for speaking and writing the "right" way, whether we are being held accountable to status dialects and prescriptive standards of edited prose or to affiliational social and cultural dialects.

So our difficulty is to develop a balanced view of linguistic orders that respects their tremendous power in creating common understanding while still unlocking the potential for more knowledgeable, reflective, skillful, critical, creative practice that participates in the contingent and evolving nature of language.

\section{CREATING ORDERLINESS OF LANGUAGE}

Literate interaction is transacted over the page, on the computer screen, and on the inscribed surface where the writer places words for readers to find them and engage with in the kinds of social and cognitive work discussed in the prior chapters. Yet the order we create in each textually-mediated interaction is not a spontaneous assemblage of newly created parts. It depends on the order of inscription symbols that has developed over time for each language. This written code typically indexes a related spoken language that is often (but not always) familiar to the user; however, this written code also then develops characteristics diverging from the spoken language. The independence of the written language from the spoken is indicated by such obvious logographic features as conventions of spacing and punctuation, but also by such subtle features as non-phonetic spelling indicating word histories or semantic relations, and syntaxes only decipherable on the page and not by ear (Harris, 2000).

The order of words we create in each utterance depends on communally shared orders of words available for our use and principles and practices for assembling them in ways intelligible to others (for historically grounded accounts of the emergence of linguistic patterns, see Bybee, 2010; Hopper \& Traugott). The need for mutual intelligibility puts pressure towards normalization. Patterning allows us to create more variety with fewer linguistic elements and allows combinations that are easier to understand, in contrast to using random variation with no regularities to aid formation and interpretation. The greater familiarity and depth of knowledge a writer has with the language or languages shared with the readers, the more resources the writer has at hand, the larger set 
of choices, and likely the greater ability to reflect, compare, and choose among options. Linguistics, philology, and lexicography have taught us much about the resources we have available and the logics by which these resources can be organized. The knowledge they have made available forms a useful part of the education of each writer, revealing a deeply subtle and delicate instrument of expression.

Yet the centrality of written symbols and language as the medium of written communication may mislead people to mistake knowledge of the medium to be the whole craft of writing-leaving all else to mysteries of artistic genius. Such an approach can lead to two dialectically opposed forms of fetishismof unregulated imaginative genius or of obsessive rule-seeking. Both forms of fetishism detach language production from the social processes that bring language into being and vivify its use. With genius alone we have only the privacy of the individual imagination as a motivating source and an organizing power with no sense of the interpersonal force of language. With regulation alone we have only knowledge of the tools of language, without a strategic sense of when, where, and why to use them. We only have collections to no purpose.

The sources of the orderliness of language have been attributed to the sacred origins of language, the nature of language, the nature of human sound production and reception, the nature of the mind that produces and understands language, the biology of breath and vocal production, the nature of inscription systems, the social processes that create social cohesion and alignment, historical accidents, and the historical production of regulatory texts and institutions (often associated with schooling, publication, and record keeping). In fact, speculation over the nature and origins of language and the attempt to understand the orderliness of language are some of the earliest forms of knowledge fostered by literacy, as written language presented puzzles of how best to accurately inscribe the spoken language, how to speak accurately what has been written; further, written language provided a stable object to collect, organize and study. Writing language down provides the opportunity and need to discover and regulate its orders. Early uses of writing for government and financial record keeping created exigency for orderliness and regularity. The early use of writing in transcribing the divine word provided exigency for accuracy of transcription and oral performance as well as interpretation (Bazerman $\&$ Rogers, 2008a, 2008b; Prior \& Lunsford, 2008).

While no definitive, fully-evidenced story has emerged concerning the origin of language and the orderliness of language, it seems likely to occur at the intersection of physiological, cognitive, sociological, linguistic, and historical processes, for each seem to present a strong prima facie case for influence. Spoken language necessarily occurs within the physiological limits of human 
voice production and control along with aural discrimination. The palette of spoken language follows the volume and pitch range of our production and reception. Similarly, the typical size and differences of written characters matches our visual discrimination at about an arm length and the fine motor control of writing implements at the same arm length. Our cognitive processes of memory, categorization, and selection in the moment of use seem to ensure that we will impose order on language. Sociological processes of creating coalignment, mutual understanding, and group cohesion would strongly suggest that orderliness, local standardization, and typification would emerge out of the need to be understood by others. We would not expect it to be any different: since spoken and written language were developed by humans, it is reasonable to expect that the media of expression would match our physiological, biological, psychological, and social capacities, and would carry out functions that would engage all these capacities.

\section{KEEPING LANGUAGE ORDERLY: HOUSEKEEPING AND PRESCRIPTION}

Much of the development of language is lost in pre-recorded time (writing of course is the key instrument for making a record of time). Yet literacy has influenced the need and opportunity for orderliness and regulation. The emergence of literacy had an effect on gathering and organizing what we know, which in turn had a regulatory effect on future productions. Print and the broader circulation of texts extended the need for greater regularity. The association of language with nation states and the rise of education systems based on standardized literate languages led to further ordering of language forms, training of users, and regulation of practices. The historical emergence of regulatory texts, such as grammar books and dictionaries, became essential tools of editorial, educational, and social prestige processes, providing strong means for language codification. All these ordering forces will be embodied in the received language, there for us to discover and make sense of as we grow up.

Without conscious ordering and various social mechanisms for maintaining consistent order, language, both spoken and written, tends to evolve within generations, perhaps faster. Consider how rapidly vulgar Latin in creolization with other languages formed the varieties of Romance languages-each of which has its own pull of differentiating dialects that have persisted despite national political and educational regulation, such as we see in the Spain where not only the Gallic Catalonian resists Castilian hegemony, but Galacian sits both geographically and linguistically between Portugal and France (which 
have each centralized their own dialectical Romance varieties under national and educational regulation). Asturian, Leonese, and Aragonese and others also maintain some distinctive linguistic status. Even the written and learned Medieval Latin rapidly evolved in spelling, grammar, and vocabulary (following the transformations of dialect) until regularization to classic norms was enforced through schooling in the Renaissance.

Prescriptive normalization has been especially intense for writing in the last few centuries, supported through reference books, school books, school practices, national linguistic academies, publishing and copy-editing standards, broad circulation of documents, and other devices. The force of this prescriptivism is troubling to a scientific linguistic point of view on several grounds. Since the time of Saussure (1916/1983) and Bloomfield (1914) linguistics has adopted a descriptive rather than a prescriptive stance, to reflect actual uses. Second, following Bloomfield, linguistics has taken spoken language as its primary data, seeing the spoken as more natural. Third, following Saussure, linguistics has largely (though not exclusively, particularly recently) pursued synchronic orders, removed from particular time and particular instances of use. Nonetheless, the process of prescriptive ordering is a deeply historical one, with formation of institutions to influence historical processes (often to resist historical change) in order to regulate uses, particularly since the advent of writing.

Writing in itself brings systematicity and regulation in the order of signs used to transcribe language, as has been studied by numerous scholars, starting with Gelb (1952) and more recently Daniels \& Bright (1996) and Coulmas (1996). Scholarship on language systems highlights the differences in principles and form by which language has been transcribed from iconographic and hieroglyphic to syllabic and alphabetic. We can see the very impulse towards creating language studies as a communal attempt to make orderly sense of the rich and expanding resources of language. Most of the history of such inquiries has something of a housekeeping impulse, whether accompanied by the prescriptivist fist of social authority and sanctions or the velvet glove of the helping hand. Even purely descriptive linguistics as practiced in the last century (adopting a hands-off orientation that requires substantial training in professional objectivity) still relies on a belief that the order is there to be found, and that discovering and articulating the order that is already there in nature can help us learn, preserve, and understand the dynamics of the language. Despite the descriptivist stance of most theoretical linguistics, we still find regulatory, normalizing, or even prescriptive grammars, orthographies, and dictionaries remaining at the heart of our educational, editing, and professional writing practices (even to the point of now being embedded in the software by which we now typically write). Language is too large and complex for us not to make 
order of for our own use and to facilitate group communication. If language production appears frequently as fully spontaneous, it is only because we have internalized so much of the order that we can deploy it skillfully and rapidly in response to situations we perceive ourselves to be in.

\section{LEARNING TRANSCRIPTION}

The richness and complexity of language presents an organizational problem for the language learner, as the child must make sense of all the phonological, prosodic, interactional, lexical, and semantic information in her ambient linguistic environment and coordinate that with her own means of production, whether the child is aided by a specific neurobiological language device as proposed by Chomsky (1965) or the child's brain creates emergent orders in the interaction with learning as argued by Bates and Goodman (1997 and 1999). Learning written language also requires coming to terms with systems built on histories and practices of regulation and prescription. Even as children become aware of the social functions of writing they also are introduced to the ordered symbols of their cultural legacy. In alphabetic languages this is taught through devices such as the alphabet song, alphabet bestiaries, and normative phonics (even though the letters may have only a loose approximation for the phonetics of the language being transcribed.) These orders as well are observable in the ambient communicative universe, as children experiment with form as a means of expression.

Studies of emergent literacy present complex stories of children attempting to make sense of, learn, and deploy the literate behaviors they see around them, with highly particular, local, idiosyncratic personal constructions by children embedded in local circumstances, but which also triangulate towards normalized uses of culturally ambient forms (Rowe, 2009). Letter formation and invented spelling at first are only loose approximations to the standard, for example, but over time normalize through a combination of personal regulation to achieve observed forms and external regulation of schooling and correction by adults, peers, and software (Sharer \& Zutell, 2003). Similarly the available orders of syntax and morphology become to varying degrees normalized, particularly as associated with advancement through schooling and school tasks. Schoolbooks, self-help books, and other guides introduce and reinforce forms and practices. Similarly students and other writers in development are introduced and normalized to the genres that form the repertoire of the school, the workplace, and social life-each with their separate methods of induction, modeling, and correction. 
For people writing in alphabetic and syllabic languages where the symbols are limited and abstracted from meaning units such as words, these principles are imbibed early in their training and are not necessarily a matter of reflection or subtle expressive choice (except when perhaps defamiliarized as in some poetic contexts or writing in dialects). Depending on the language and the instability of the phonetic correspondences, alphabetic transcription may be variously problematic for literacy learners, but is usually resolved by primary school years. Spelling may also contain etymological information as well as morphological features tied to grammar and syntactical issues. These may be called to students' attention as they are learning more advanced spellings and are being held accountable for grammatical correctness. Even when moving between two languages using an alphabetic system with Roman characters, there are challenges of phonetic mapping and spelling-issues highlighted for example, when singers must perform scores in different languages. In some consonantal syllabic languages without vowel pointing (such as dialects of mid-Eastern languages), understanding and using the transcription system properly is intertwined with lexical, morphological, and syntactic issues as well as meaning, such that a high level of expertise is necessary for accurate transcription and reading. Further, in languages which have complex mixtures of iconic, pronunciation, and disambiguation elements in the characters, such as Chinese, the study of characters and their differentiation remains a complex concern throughout one's literate life, intertwined with extended vocabularies, meaning potentials, allusions, and fresh combinations. So in choosing or forming a character a writer may be invoking cultural histories, textual resonances, regional differences, or meaning associations of the sort that in other languages occur at the word, phrasal, and intertextual levels. So while in some languages the orderliness of the writing (or character) system is relatively unproblematic and thus usually not foregrounded, in others distinctions within the transcription system remain important carriers of meaning and thus call for conscious attention.

This learning of the transcriptional orders of language goes hand in hand with developments of visual perception and discrimination, as well as motor and attentional control, for both reception and production. Eyes must learn to focus on small symbols with minor stroke differences, and these must be perceived (at least in alphabetic, consonantal, and syllabic languages) as sound equivalents. Fingers must come under control in coordination with visual feedback and productive intentions to produce letters and words. Dots and punctuation marks must be noticed and seen as worthy of attention in production and reception, along with morphological markers. Such issues as placement on page, genre markers of format, and sustained attention for multi-clausal statements 
and logical relations, continue throughout one's maturation as a writer, as one encounters new forms of suspended sentence, appositional phrases, sentence rhythm, rhetorical figures, and the other elements we associate with advanced style. Deficits caused by injury and aging may also require adjustment of the most basic regularized motor production skills and visual recognition, as well as to the more advanced cognitive skills necessary for attending to larger organizational structures.

\section{WORDS AND LEXICAL ORDERS}

It is seductive to imagine that the lexicon of any language, in the manner Saussure proposes, is an orderly system of differentiations of paired or neighboring terms. In pursuit of this vision, various language reformers have proposed creating more orderly and univocal vocabularies for a language, where each signifier designates a unique signified and each signified has a unique term; further, in some systems such as Bishop Wilkin's (1668) system of real characters only things he considers to be true are designated signifiers and no signifiers are afforded anything that might be considered phantasmagorical. For some languages, national academies and other regulators attempt to keep the vocabulary orderly and spelling constant in the face of neologisms and incursions from dialects or other languages. They also may attempt to protect signifier-signified relations from ambiguity and duplication. These academies have their origin in the Florentine Accademia della Crusca, founded in 1582, which provided the model of the Académie française founded in 1635. The Vocabolario della Crusca first published in 1612 was one of the earliest national dictionaries and current editions still maintain an authoritative role in defining the official language, as does the Dictionnaire de l'Académie française (first published in 1694). In 2012 there were over 85 such bodies around the world, many of them having official government status (List of Language Regulators, 2012).

Even in officially unregulated languages like English, dictionaries provide censuses of the common stock of language. English has no single official dictionary, but since Samuel Johnson created his A Dictionary of the English Language in 1755, several competing dictionaries have shared authority for British and American versions of this language. Although most contemporary lexicographers consider themselves to be descriptive rather than prescriptive, the dictionaries they produce limit and focus meanings, establish authoritative spellings, and slow the adoption of neologisms. Dictionaries put some order into the welter of social, historical uses and roots of words, variations of spellings, 
multiple meanings of words, and relationships of words bearing having related referents. In the face of the fecundity of language extending processes, they create logical distinctions among words, and put order in the complexity of features and dynamics associated with words. The more authoritative of these dictionaries are used to regulate print and educational contexts, and copy editors, teachers, examiners, and similar language guardians have the task of enforcing not only words, but spelling and grammatical forms and usage as well. Dictionaries and related references such as thesauri also help guide writers among the alternatives to make choices that are not too idiosyncratic and are intelligible to others. Yet, even though authoritative dictionaries can slow word change and can provide a reference point, they cannot stop innovation and change, through borrowings from other languages, neologisms, simplifications, hybrids, and the need to respond to new concepts and objects on the landscape. New words and locutions also serve ever-present needs for social affiliation, differentiation, and saliency.

The complex and evolving relation between meanings and the available words is reflected in the long discussion of the relation of lexicon and semantics that bridges linguistics, psychology, and philosophy. Within linguistics the idea of creating a fully ordered semantic field, or possible meanings against which words can be measured, has turned out to be quixotic. As languages grow and cultures change their knowledges, the semantic possibilities change and extend both for individuals and members of the community. Lexicon and semantics grow through both an inward conceptual expansion and a probing outwards into the world to identify possible things to be indexed and turned into meaning through the form of words, often using shards and analogies of previous words and meanings. As a result dictionaries become baggy collections where disparate meanings and word relations are stored and made evident. Yet for reference purposes this disorder is contained within highly ordered systems of representative devices, such as the conventionalized form of dictionary entries and the arbitrary arrangement of alphabetic order, itself based on the oddities of spelling and conventional ordering of letters in any language.

Specialized words and meanings of particular fields, whether theological, sports, criminal, or academic also expand in complex ways the resources of a language. Chemistry provides a very striking example, as it has developed a highly technical esoteric nomenclature for the naming of elements and compounds and has transformed general vocabulary words to technical ones, such as bonding. Unless one is to some degree a part of the epistemic community, one has little idea of the meaning of words and the relation to others. Learning the lexicon goes hand in hand with learning the theory and knowledge of the field. Within these specialized worlds, authoritative bodies may periodically 
attempt to clean up and order what they know, disambiguate terms, and lay out theoretical and concrete relationships of terms. Again consider the example of chemical nomenclature where each word part conveys a specific and fixed meaning about constituent elements and molecular form, which in turn exhibits familial relations among compounds. Even here, however, changes in knowledge and theories can destabilize tightly tuned systems of nomenclature.

When we are writing within such carefully honed domains of ordered meanings and words matched to them, our meanings are determinative of our words and our words of meanings. We must be carefully attuned to always getting the word choice right, on penalty of being viewed as ignorant and unpersuasive-as well as not being understood accurately. Expressing new meanings or meanings that cut against the grain of the knowledge system can be difficult, if not impossible. On the other hand, when writing across domains or in less highly constrained epistemic arenas, we have at our call such a heterogeneous collection of words that choosing the right word to evoke in the reader the meaning we hope for can be a puzzle.

What this means for the developing writer is that expansion and refinement of vocabulary is a constant challenge, even late into one's career. Writers look to discover the relations among words, how they evoke meanings in combination with each other, the meaning worlds they take the readers to, and how words may be applied to particular circumstances to identify particular states of affairs. Often vivid meaning is most effectively accomplished not by exotic or unfamiliar terms but by apt choice among the most familiar stock, but in a way that freshly animates meanings, so people are attentive to the particulars evoked rather than normalizing the message into the familiar and unremarkable.

\section{SYNTAX AND GRAMMAR, ORDERING WORD RELATIONS}

As with the different lexicons and semantic possibilities of each language, each language also offers a different range of morphological markings and syntactic relations. Verb morphology, for example, can provide strikingly different possibilities for expressing time relations as well as number, mood, voice, and epistemic evaluation. With respect to only one of those dimension, verb tense, some languages offer only limited options, such as a simple present, past, and future, while others offer finer distinctions such as in the last few minutes, earlier today, the remote past, or dream time. Some languages offer perfect or continuous markings to express completed or ongoing events within different time frames, and so on. 
Similarly, the syntactic patterns available in each language have consequences for what relations among lexical items are expressible and with what emphasis (Slobin, 1987). As prescriptive grammars attempt to regulate and hold constant standards of correctness, they also work to restrict meaning potentials, but writers driven by meanings may seek to stretch the boundaries of regulation. This is visibly so where written dialects and registers may carry in their morphological and syntactic features messages of social affiliation or reference, stance, power, cognitive and affective domain, or other salient meanings. Correctness according to the rules of reference book is only authoritative in those domains which take them as authoritative, such as in school or formal edited publications. Prescriptive grammars articulate and make more predictable the morphological and syntactic systems of languages which may evolve and lose distinctions without regulation. For example, even with attempts at regulation, the subjunctive mood is vanishing in American English and becoming less recognizable to readers. From the perspective of writers, familiarity with the regulated and prescribed morphology and syntax provides a range of expressive potentials which may be mobilized, but this must always be tempered by an understanding of changing usage and what is likely to be familiar and intelligible without undue cognitive strain by readers.

While the study of grammar and debates over the orderliness of language go back at least to the Alexandrine grammarians in the third century BCE, the authoritative prescription of grammatical rules does not seem to have emerged until the late medieval school which changed the curriculum from immersion in classic texts to the systematic presentation of principles of language. The earliest popular grammar codification was the Doctrinale of Alexander of Villedieu, written in 1199 in verse as a mnemonic. In English Robert Lowth's $A$ Short Introduction to English Grammar (1762) appears to be the first widely used prescriptive grammar. The rather late arrival of prescriptive grammars is tied to standardizing practice for education or publishing purposes. While ordering processes of language arise from practice (whether driven by psychological, social or cultural forces), self-conscious linguistic regulation is only a late comer to help standardize practice with particular historical problems, such as Medieval Europe being confronted with an influx of ancient Greek and Latin texts or political desires to impose a standard educated dialect over a large region. The orderliness of language exists prior to the regulation, and the attempt to meet regulated norms is rarely a core motive of writing, except in school examinations or contexts where one may be severely stigmatized for using non-elite forms.

There is also a history of advice for larger units of text organization from paragraphs to whole texts. Books of models and forms for letters, going back 
to the medieval ars dictaminis and Renaissance style books, but these are always in the form of advice, potentials to be mobilized at the writer's choice. In schooling sometimes these forms are taught (such as modes of comparison, contrast, narration), and some examinations presume certain forms as the most effective solutions-five-paragraph essays. Similarly, examinations for career advancement, such as in the Chinese Imperial examinations, can enshrine expected forms, such as the eight-legged essay. Training for particular professions sometimes includes practice in stylized versions of genres currently in use, with some conservative reifying effect in the face of evolutionary forces in actual practice. Economic or legal stakes, and even legal regulation, can also be extremely strong in defining, for example, what must appear in a patent application, contract, or other document with legal and contractual force. Forms and questionnaires also represent attempts to regulate representations, but even such forms vary and evolve.

\section{THE EDUCATIONAL USES OF RULES}

Evidence indicates that direct instruction in language rules outside of the context of need and practice has dubious value for first-language speakers (Graham, 2006; Hillocks, 1986). Significant gains have been observed in student writing when they are relieved of the pressure of producing "correct" language. Indeed it is unclear how much conscious or explicit invocation of rules usefully occurs during composition by competent writers familiar with a language, at least until the later stages of sentence crafting, editing, and proofreading.

On the other hand, it is impossible to produce utterances longer than fragmentary phrases without a sense of the orderliness of language; moreover, some highly-skilled writers use conscious knowledge of lexical, grammatical, and syntactic distinctions and patterns to extend their expressive potential. The extent to which writers gain that sense of orderliness from neurological constraints, interactional experience, internalized early learning, wide reading, or other mechanisms is still uncertain, as is how that knowledge is best invoked in instruction, composition, revision, and editing. What is clear is that historically our explicit documentation and regulation of the orderliness of language came after our ability to use writing. Thus the pedagogic strategy of attempting to habitualize, normalize, and regulate the repetitive elements of language apart from the acts of creating valued meanings may have human processes backwards.

The motive from the learner's perspective is always to make meaning, or at least master the tools of meaning so as to become a more competent meaning- 
making creature. As G. H. Mead points out, we regularize ourselves to an intelligible social identity in order to be understood and understandable by others. We then look on this identity and from it construct a sense of the self, the self that resides in social relations to others. Therefore, the learner's sense of the self as a writer depends on how he or she is induced into these orders. If these orders are learned and practiced within a wider set of meaning-sharing practices, the learner comes to recognize a self that can create meanings through skillful and technical use of the tools of written language. On the other hand, if the learner experiences these orders as something to be followed for oneself alone, and comes to see his or her primary competence as the ability to follow the rules and produce correct utterances, the learner will have confidence only to produce the most conventional and normalized of utterances, always under the anxiety of failure of propriety.

Any detail or difference of language can be the bearer of meaning. Language users have an incentive both to create novel variations and to recover the potential of variation of those aspects of language that have become so routinized and stabilized so as to become in a sense invisible, routinely not calling attention to themselves. Indeed at the higher level of skills, such recovery and attention to detail is of great importance. Thus while letter forms are taken for granted by most of us and their recognition and production are early habitualized in children, graphic designers lavish attention to the development and selection of type-faces, whose meaning and value is only appreciated by a few, although design consequences may be felt subconsciously as comfort and discomfort by the inattentive reader. The detail of definite or indefinite article can have important meaning consequences if one is paying attention with a level of precision, as is highlighted in Dorothy Parker's reputed quip about Lillian Hellman "Every word she writes is a lie, even $a$ and the." We may say the same about sentence rhythm, sequencing of lexical images, deployment of prepositions. The more skilled the writer is, the more the writer attends to such details with care.

Variations that call attention to themselves by violating conventional orders are even more visible and can contain strong effect to wrench messages outside propriety. If rules and orders become habitualized, routinized, and engrained as moral order then every attention-getting and novel meaning-making variation may be viewed as transgressive or even repellent. The taboo borders of vocabulary, politeness and face devices, and syntactic familiarity put constraints on individual expressiveness, but they also create the possibility to experiment with shocking meanings and messages that are just over the border.

The orders of language we teach are themselves artifacts of literacyproduced, recorded, and spread through literacy and largely arising from literate 
practice, such as creating relations between phonology and letters, making dictionaries, or writing grammars. Much of what we teach as order does not come from the simple need to create common language, but is tied to histories of political power, control of educational systems, centralization of printing, class stigmas, xenophobias and ethnocentrisms, hypercorrectness of regulators, linguistic ideology, reformist zeal, or idiosyncrasy. Insofar as these then establish a public standard they are real, but they are freighted with much baggage which the learners may not be aware of and which may influence their perception of themselves as writers.

Consequently, teaching and learning of linguistic orders must always draw on and serve the learner's sense of meaning making; if language learning becomes purely a matter of forming habits without purpose, then the learner will have little motive beyond obedience and will not know what the learning is good for, except rote repetition or fetishized evaluation. 


\section{UTTERANCES AND THEIR MEANINGS}

Meanings are constructed situationally by the participants in interaction, as they construe intent in each other's uttered words. A well-known story (said to be a favorite of both Vygotsky and Bakhtin) tells of a group of sailors having a nuanced exchange by repeating the same expletive to each other, but with a different intonation and timing at each turn. This polysemousness of words is equally to be found in an office memo announcing a change in reporting procedures that leaves the recipients wondering what the real meaning is-from enacting a corporate shake-up, to disciplining a co-worker, to a power-grab by a manager, to simply creating an efficiency. Much water-cooler time may be devoted to examining the nuances of expression or sharing other contexting information until a stable social meaning is agreed on, which will then guide the behavior of all concerned. To put it explicitly, meaning is not a property of language in itself, and is not immanent in language. Meaning is what people construe using the prosthesis of language, interpreted within specific contexts of use. To understand meaning, we need to take utterance and people's construal of utterance as our fundamental units of analysis.

\section{VOLOSINOV AND HIS CIRCLE'S PROPOSAL FOR AN UTTERANCE-BASED LINGUISTICS}

Volosinov in Marxism and the Philosophy of Language (1929/1973), foreshadowed by comments in his earlier work on Freud (1927/1987), argued that linguistics should be grounded in utterance, rather than in the formal structure of language. Utterance was the natural unit of speech and communication, with each utterance taking shape within a recognizable form (that is, a speech genre), directed to a specific audience (what Bakhtin, 1984a, 1986, was to call addressivity), and in response to prior utterances. Volosinov's St. Petersberg colleagues during this period further elaborated this utterance-centered view of language. Medvedev (1929/ 1978) placed utterance-based genres at the center of sociological poetics. Afterwards, in the 1930's and later, Bakhtin pursued genre, addressivity, and responsivity to other utterances in relation to the novel and other literary texts as forms of ideology and consciousness. In the 1950s 
Bakhtin developed a social theory of speech genres as situated utterances, but his most widely-circulated essay on the subject "The Problem of Speech Genres" was not published in Russian until 1979 and English until 1986.

The view of language shared by Volosinov, Medvedev, and Bakhtin is dialogic, grounded in human interchange. Utterances respond to prior utterances, so that "each utterance refutes, affirms, supplements, and relies on the others, presupposes them to be known, and somehow takes them into account" (Bakhtin, 1986, p. 91). In responding to prior utterances, each new utterance transforms and further populates the landscape within which new utterances will be added. As actual situated communication, these utterances (and the sequences of utterances they refer to) rely on and carry forward personal, interpersonal, institutional, sociocultural, and material histories. They enact relationships and social forms of life within the actual circumstances of life. They are charged with emotions, motivations, stances, evaluations, and concrete intentions, which color the specific semantic content of communications and provide the basis for interlocutor interpretations of each utterance and the overall unfolding of events. The utterance is a process, a form of co-production, a circuit that is complete only when actively produced and actively received. Volosinov pursues the dialogicality of language in the last part of his book on the philosophy of language through a technical analysis of reported speech. Explicitly representing the words of another and adopting a stance towards them overtly places the new utterance within an historically emergent social dialogue. The syntactic and grammatical means a language provides for reporting on and taking a stance towards another's language supports the forming of particular kinds of social relations and interactions that unfold over time in conjunction with linguistic change as a part of changing social relations.

This analysis of language to reveal specific social meanings created through the situated use of evolving language sharply contrasts with dominant forms of linguistic analysis initiated by Saussure who decomposed langage (language) into langue (the system of language) and parole (any particular situated use of language), and taking langue only as the concern of linguistics, because parole (and by extension langage that united langue and parole) was too multifarious, multi-dimensional, and multi-causal to lend itself readily to scientific analysis. Likewise, Saussure distinguished synchronic (in the single current moment) analysis of langue from diachronic (over time) analysis, taking only synchronic analysis as the proper scientific subject of linguistics. Saussure, through these two moves, directs the study of language toward the study of an abstract object out of time, out of interaction and use, and not subject to the changes brought about by individual situated use and invention. 
Volosinov criticized Saussure's approach by saying that such a concept of langue does not correspond to the actual appearance of language in the world, which is as a constantly evolving set of uses within particular situations. The only place such an abstract construction of a langue could actually exist would be in the consciousness of an individual, but that individual when confronted with an actual communicative situation adapts and improvises to convey a meaning directed toward the addressee (p. 85). Volosinov expresses the mutability and purposeful use of language by noting "what is important for the speaker about a linguistic sign is not that it is a stable and always self-equivalent signal, but that it is an always changeable and adaptable sign" (p.68). He continues to consider the perspective of the listener by noting, "the task of understanding does not basically amount to recognizing the form used, but rather to understanding it in a particular concrete context, to understanding its meaning in a particular utterance, i.e., it amounts to understanding its novelty and not to recognizing its identity" (p.68).

Volosinov's critique of structural linguistics has been echoed by many since, including Kristeva (1980), Todorov (1990), Harris (1981, 1987), and Hanks (1996). Others have more recently attempted to explain aspects of even such fundamental organizing elements of language as grammar and syntax on the basis of interaction and unfolding dialogic sequences within real unfolding communication (Ochs et al., 1996; Selting \& Couper-Kuhlen, 2001). This research aims to understand morphosyntactic and prosodic patterns in terms of social action and social processes of organizing communication.

\section{UTTERANCE TO SPEECH ACT}

This view of meaning as construed by participants through the use of language in the course of interaction is consistent with Wittgenstein's examination of language as meaningful in specific contexts, where participants take up meanings in the course of activities rather than directly translating meaning from an abstract system of language with stable semantic referents, existing outside concrete historical interactional events. As is well known, Wittgenstein's (1958) adoption in Philosophical Investigations of a situated view of language embedded in interactional events reversed his more youthful project of creating a mathematically consistent logic in Tractatus Logico-Philosophicus (Wittgenstein, 1922).

Austin and Searle, in developing the concept of speech acts, sought to elaborate just what this action-oriented view of language might mean. Austin (1962) begins the early lectures of his volume on How to Do Things with Words 
with an analysis of the most salient kinds of actions accomplished through words, such as making a bet or naming a ship. This analysis leads him to identify all the contextual and attitudinal conditions to be met so that action would be interpretable, trusted, and sufficient; these he calls felicity conditions. At first these have the appearance of being universal and general, as though these orders of actions could be universal and logical, apart from histories, local circumstances, or social arrangements. However, by the later lectures he returns to a much looser definition of felicity conditions that depend on individual construal of local circumstance and particular historical and institutional arrangements that establish conditions. Additionally, in the early chapters of his analysis he distinguishes between locutionary meanings and illocutionarythat is, between the action part of the utterance and the representation of affairs, which we might call the semantic meaning. However, by the closing lectures he identifies representation itself as a speech act, and therefore dependent on the local construal of conditions, social positions, and interactive trust. Thus even the successful representation of states of affairs depends on local situational and institutional histories and conditions: "The total speech act in the total speech situation is the only actual phenomenon which, in the last resort, we are engaging in elucidating" (original emphasis, p. 148).

Searle, however, in his book on speech acts (1969) does not turn back from the attempt to domesticate the social and historical unruliness of speech acts into a rational order. Searle reduces rules of felicity conditions into a logical calculus for each of the major categories of acts, and in further work continued to put this into formal logico-mathematical calculus, as though Wittgenstein were not as revolutionary as purported, but had simply identified another dimension of meaning which could be brought to full and stable order in its own logical terms outside of human time but with the purity of mathematical space (Searle \& Vanderveken, 1985). Similarly Searle maintains the integrity of the locutionary act as a place where logic also holds sway in the representation of things. He does, however, later $(1983,1992)$ introduce a concept he calls "the background" which refers to the knowledge, tendencies, dispositions, abilities, and capacities people have through their experience of living in human communities. This concept of the background opens up the possibilities of variation of human experience, understanding, and interpretation outside of the formal representation in language.

While I am in no position to evaluate the philosophic correctness of Searle's claims, Austin's account better resembles the contingent, socially changing, phenomenological, rhetorical world of human communication, where people constantly make sense of each other's words in historically evolved and evolving 
circumstances, for purposes at hand, without rigorous calculation and evaluation of claims' logical terms, but drawing on their experience and situated construal of meaning. That being said, Searle does provide insights into the dynamics of interpretation and evaluation of some of the felicity conditions that maintain for the success of acts in certain circumstances.

While Austin and Searle were concerned with short spoken utterances (of the length and character of "I bet you that ..." and "I declare you guilty of the crime of ..."), longer written texts can be understood as carrying out social acts as well, though some cautions and qualifications are necessary in carrying out the details of analysis, particularity concerning the univocality and determinability of the act (see Bazerman, 1994b). That is, a long text may signal multiple acts to the readers, with some appearing hierarchically more important, and since a written text may travel to many different situations and engage various users, the perlocutionary effect (uptake) of the acts may vary even more greatly and unpredictably than in face-to-face circumstances. Thus the interpretation of the speech acts in an extended written text may be more difficult and equivocal. Nonetheless, each user will find the texts accomplishing or failing to accomplish specific acts. Genre recognition then provides means for typifying and recognizing the meaning and import of texts as well as the situation and activity the texts are part of. As people come to use and understand the textual artifact in particular ways, the genred text becomes a crystallization of an action, with the consequence that writing an article or finishing reading a novel may become an end in itself (or the object in activity theory terms - see Chapter 3, this volume). As with all mediating artifacts that serve as tools for accomplishing participants' objects, while genres may suggest and support particular typical objectives, they can be used flexibly depending on each participant's personally framed objects (Cole, 1996; Wertsch, 1998). Yet, through the sufficiently mutual alignment achieved through the mediating artifact, speech acts are accomplished, for people come to some sense(s) of agreement on the meaning, interactional force, and consequences of actions.

Genre, by shaping the roles of participants in a situation, also frames the addressivity of those texts that realize the genre. As Volosinov comments, "The word is oriented toward an addressee"(Volosinov, 1973, p. 85). This orientation to communication with an external audience in a specific situation brings about a transformation of the internal word to a dialogically interpretable utterance and act. As Volosinov explains, "the word is a two-sided act . . . the product of the reciprocal relationship between speaker and listener, addresser and addressee"(Volosinov, p. 86). This dialogic situation, the emergent inner impulse, and the need to be situationaly effective, "determine-and determine 
from within, so to speak-the structure of an utterance" (Volosinov, p.86). Bakhtin specifically ties addressivity's determination of utterance structure to genre, which enacts recognizable and familiar roles, relationships, and interactions: "Each speech genre in each area of speech communication has its own typical conception of the addressee, and this defines it as a genre" (Bakhtin, 1986, p. 95).

While texts may arise to express the needs, character, purposes, and thoughts of individuals, how the texts express themselves and the social presence they take on are framed by the situation, roles, and actions they are engaged in. An immigration official inspecting applications adopts the values, evaluative practices, and decision-making concerns appropriate to the role and the document being inspected. Insofar as the official varies from these generic understandings, he or she may be said to be acting unprofessionally, violating expectations of appropriate situational action. Even when individual judgment is a central expectation, such as intellectual judgment involving advanced theoretical knowledge and critical evaluation, perhaps in a symposium response or a journal review, the idiosyncratic message still must be expressed appropriately to the genre, framed within the evaluative practices, empirical criteria, and theoretical constructs appropriate to that line of work and constructively carrying out the collective work of the domain with awareness of the evolving situations of the collective work. Additionally, the comments need to reflect the respect, status differentials, and acceptable dialogic stances towards colleagues, maintaining professional face of participants.

\section{SOCIAL FACTS}

The acts accomplished by genred utterances in turn establish social facts and reinforce all the underlying social facts on which the new act depends. Social facts are those things people believe to be true, and therefore bear on how they define a situation and act within it. The sociologist W. I. Thomas (1923) states it so: "If [people] define situations as real, they are real in their consequences." Thus the worlds successfully evoked and enacted in the genred utterances can become a kind of self-fulfilling prophecy (Merton, 1948), or a deictic evocation and shaping of a life world (Hanks, 1990, 1996).

That documents create social facts is most easily seen in texts like contracts, applications, and business orders. In such cases the text provides the basis for further action (e.g., job interviews will be scheduled and products will be shipped) and holds parties accountable for the commitments made in the text 
(e.g., that I will complete the contracted work or that I will accept delivery of the product ordered). However, less obviously behavioral statements can also be seen as acts and consequent social facts. As Austin and Searle both point out, assertions are also acts. Assertions do not necessarily need to be taken as true to be taken as a social fact that they have been asserted. If an appropriately credentialed member of a profession presents a controversial research paper to a professional audience, delivered in an appropriate form and forum, then people do not have to accept the claims as true for them to recognize that the claim was made. The intellectual landscape of that profession will have been changed to the extent that the author has gotten people to attend to that claim.

Indeed if the statement is extremely controversial, then there will be many consequences and further acts from the social recognition that the person has made this claim. It may become very difficult for the controversialist to erase the opprobrium that comes from the social fact of being associated with especially dubious claims. It may even be the case that the author never hoped for agreement, but only wished to challenge current even views and create a discussion. In that case, the author would have created exactly the desired social fact. Every text that is attended to or otherwise finds place on the discursive landscape can be said to create some kind(s) of social fact, even if only to leave an objection on the record.

Of course, the textual act might not be recognized for everything the author would wish it to be, but then what conditions would the author have to meet in order to carry out the desired act? What new evidence or experiments would the author need to produce in order to stave off a particular objection? On the other hand, what maneuver can the opponents make to undermine the apparent accomplishment of having an experiment accepted as valid and definitive for the theory in question? These conditions that have to be met for an act to be successfully realized may be seen as forms of accountability. If a condition is not met-a legal document is not filed before a requisite deadline, confirming experimental evidence cannot be found for a chemical claim, a political claim does not resonate with the interests of the electorate-then the speech act will be called to account and fail. Of course, if the author can provide an additional account that puts the accounting back on the positive side of the ledger-a lawyer successfully argues that an extension be granted on the deadline, the chemist convincingly describes the limitations of the experimental apparatus, the politician appeals to nobler motives that bestir the electorate to rise above their interests - the speech act might still be retrieved (Bazerman, 1988, 1997, 1999a; see also Latour, 1987; Latour \& Woolgar, 1979 on facticity in science). 


\section{MEANING IN THE SITUATED SPEECH ACT WORLD}

At times the significant meaning taken up by a recipient may entail very little attention to the specifics of the message embedded in the text. A watchman patrolling a building may routinely send periodic message on a hand-held device or at a station, reporting time and location. The typical meaning is only in the routine filing of the text, and the construal by the supervisor that all is well. The message is minimal and hardly attended to, unless there is some anomaly, lapse in reporting, or non-routine elaboration which may lead the message to be examined in great detail, even concerning the exact time or variation in phrasing to be matched to other information from security cameras, reported information by others, broken windows, and other crime-scene evidence. Then the message or its absence may be construed in a way so as to reveal new meanings.

Much of what we communicate on a daily basis demands only a modest amount of attention, with much of it directed toward the adequate, timely, appropriate fulfilling of the expectations of a genre: we have filled in the government form with a valid address and we have signed it correctly, an email from a friend tells us all is going well in perhaps more detail than we want to think about at the moment, we skim the main bulleted points in the executive summary of a report and follow up on only a few points which touch our interests. Readings are often perfunctory with less information passed than we might imagine.

Yet under some conditions we do read more attentively and have high expectations of the detailed content to be conveyed through text signaling. At times these expectations may have to do with the density of information to be conveyed by the document, sometimes with the anticipated pleasures or rewards that attentive reading will reveal, sometimes with importance in mediating important contested social meanings requiring extensive interpretation, and sometimes with important interests at stake. The first kind of careful reading from text density, we might archetypically see in students with textbooks, technicians with repair guides, or anyone attempting to fulfill regulations. The second kind, careful reading for pleasure, is often exemplified by literary texts, biographical narratives, or historical accounts of personal interest. The third kind, from contestation of ideas, might involve a policy deliberation or philosophic issue where we are trying to understand and evaluate each other's position to assent or offer a counterargument. The last kind, of high interest stakes, is exemplified by reading of the laws in a legal case or the reading of a sacred text when we feel as though our souls are at stake. In each of these cases we put great weight on the contents of the texts and how those contents are 
bound together in a single text. Such a commitment to the text is facilitated by a simplifying belief that meaning is carried directly through the text and its language, that language carries absolute and clear meanings, and attention to the word will get you to clear and definitive meanings.

Traditionally, theories and practices of textual interpretation have relied on such an assumption of meaning being immanent in the text. Peirce (1958) in the late nineteenth century, however, pointed out that meaning derives from acts of interpretation. Heidegger (1962) further noted that meaning was created only within the reader's life-world and was dependent on subjective positions and personal contingencies of experience. The hermeneutic circle, that suggests that every interpretive meaning is based on earlier sets of interpretive meanings, implies there is no fixed, solid position from which a single, authoritative meaning of a text can be determined (De Man, 1983; Gadamer, 1975; Shklar, 2004). Much of modern interpretive theory has struggled with this scandal of the lack of certainty and fixity of meaning.

Viewing texts as mediating situated activity, consistent with the postHeideggerian view of hermeneutics, places meaning within the life-world of actors. In the text-as-mediator view, meaning is embedded in the activities of the participants and their construction of the situation and activities; thus meaning is interactionally created between text and writer or reader-and ultimately between writer and reader through the skeletal mediation of the textual artifact. If readers and writers imaginatively construct and reconstruct meaning from the thin and fragile clues of texts, then meaning is an evanescent phenomenon. Meaning exists only as long as readers and writers attend to the text and only in the ways they attend to the text for the moment. Meaning evolves as readers move through a text or retrospectively look back on texts read.

The importance of attention to the text, its specific contents and phrasing, and the meanings mediated by it, consequently, presents challenges to an utterance perspective which locates meaning in the writers and readers rather than having meaning immanent in the text or language. We will now try to develop an account of meaning from an utterance perspective that warrants close attention to the details of a text and which can suggest how texts can serve to co-align writer and reader on specific contents, reasoning, and meaning despite their individual and socially patterned differences in experience, cognition, attention, and interests. Without such an account it is hard to justify a pedagogy of attention to the text, a responsibility of readers to read carefully, and the legitimacy of social systems that rely on hermeneutic practices, such as the law. Unless we have a persuasive account of why it is worth paying close attention to a text, we have little motive to pay close attention to one another's words and little basis to hold others to account for inattentive readings. 


\section{MEANING FROM AN UTTERANCE PERSPECTIVE}

Some thought has been paid historically to the problem of how texts or language mediate alignment of meaning across minds. The dependence on participant understanding was recognized in classical rhetoric by such concerns as the nature and role of enthymemes, the character and disposition of audiences, figures of thought, and the psychological underpinnings of arrangement. Persuasion, as a movement of the mind, was seen as dependent on individual sense-making even though this dependency isn't always made explicit for analytic scrutiny, as rhetoric remained largely focused on the rhetor's strategy embodied in the text. Rhetoric's attitude toward sense making is shaped by rhetoric's origins in oral performance, which leaves no artifact (except for the occasional script or transcription that Plato has so much fun with in the Phaedrus). Oral rhetorical performance confronts rhetors with embodied audiences whose minds they have to move, and confronts audiences with embodied rhetors who appear to be thinking about one thing and then a moment later thinking about something else. The fleeting meaning held in the rhetor's mind communicated to the audience transfigures and unites them momentarily, to be soon dissipated as thought and attention turn elsewhere. Such is the flow of life noted by the sophists.

The earliest principled attempts to develop a literate rhetoric in the medieval ars dictaminis (Murphy, 1971), to provide guidance for correspondence within the church bureaucracy, carry that same concern for socially located sensemaking, even though transmitted over distances of space and time. The ars dictaminis advise embedding the communication within social hierarchies and situations so that requests appear within well-defined social circumstances and relations, maximizing the reader's favorable sense-making orientation toward the letter and the letter writer. Proper modes of address invoke and respect institutional role hierarchies and evoke socially shaped benevolence. Other tactics strengthen the benevolence of the relationship, the good will of the receiver, and the respect granted to the reader, to make a favorable reading more likely. Further, narration serves to establish the situation-building an interpretive frame by placing writer and receiver within social positions and events that construct sense-making standpoints. Finally, arrangement is presented as psychologically motivated, modified to fit the particulars of the letter situation (Bazerman, 1999b ; Perelman, 1991).

Eighteenth-century rhetorics, aimed at facilitating participation in newly powerful print culture, are very much concerned with the problem of how the writer can use description to evoke sympathetic sense-making by the reader. 
Adam Smith, for example, caught up in the psychological conundrums posed by Locke, Hume, and Berkeley, sees sympathy at the heart of community, communication, and ethics (Bazerman, 1993b). Similarly, Joseph Priestley sees the force of description in sharing the experiences and perceptions of humankind so as to transcend the limitations and idiosyncrasies of individual souls (Bazerman, 1991). This mid-eighteenth-century concern for evoking understanding through sympathetic reconstruction, however, led to belleslettrism, as literature became the mechanism by which we were to understand each other's perspective and develop our sympathetic sense-making imagination. The turn to the literary text combined with romantic notions of genius was accompanied by an increasing trust in the words of the artist, which were taken to be meaningful and out of time, space, and social transaction. This trust in the word of the artist reinforced belief in meaning residing in the text. Much of literary criticism and literary education from the mid-nineteenth through most of the twentieth centuries, can be understood as attempts to increase the ability to appreciate what the text offers. This attention to texts culminates in the new criticism, which was originally motivated to improve student attention to texts (Richards, 1924, 1929). New criticism offered a way to unpack high degrees of textual subtlety (Brooks, 1947), but also led to an awareness of the ambiguities of texts (Empson. 1947) and ultimately to the gaps in meaning and reasoning of texts (Derrida, 1981). The reliance on the text also led to an explicit rejection by some of authorial intent (Wimsatt \& Beardsley, 1946) and readers' emotions (Wimsatt \& Beardsley 1949). Readerresponse theories, deconstruction, and a return to historicism were reactions in literary studies against the over-reliance on an abstracted text and its limitations in conveying meaning, but this has left literary studies with a scandal of indeterminacy of textual meaning, undermining the stability of the interpretive project and its allied vision of social order through cultivation of the individual's sensibilities.

Through the mid-twentieth century, the cultural trust invested in the imaginative literary experience to be found in the literary text as re-performed by the expert reader carried the implication that all texts that did not embody or evoke forms of literary imagination were less interesting, hardly requiring sense-making, and certainly not expert sense-making. Non-literary texts were considered transparent in their meanings, requiring little interpretation, imagination, or educated sensibility. Even the higher reaches of non-literary or non-humanistic disciplinary literate practices were largely treated as unimaginative. There was a minor tradition of practitioners of high prestige professional fields asserting the special imaginations of their professions-the 
legal imagination, the sociological imagination, the scientific imagination, the technological imagination, the mathematical imagination. But this always has been presented as something of a surprise and an argument for recognition of the extension of imagination in these unexpected places. We rarely hear of the dentist's imagination, the accountant's imagination, the bureaucrat's imagination, or the merchandiser's imagination—except perhaps as a joke or a criticism of bourgeois life.

\section{SENSE-MAKING IN EVERYDAY LIFE}

From the phenomenological perspective deriving from Schutz (see Chapter 4) and elaborated concretely for communication by Goffman's interactional order (see Chapter 7), however, it becomes clear how much imaginative work each person performs in understanding, aligning to, and transforming everyday situations through recognizing, responding to, and using social typifications to create sites in which people can co-align to actions and meanings. Each different potential footing for an event brings to bear interpretive and participatory sets of understandings and identifies a repertoire of expressive tools that may be appropriately drawn on. Gumperz (1992) has noted further that we use contextualization cues to signal the kind of event going on, what footing we are communicating upon, and thus the dramatic frame in which we are continually improvising our actions and in which we interpret the actions of others. However, the footing or phenomenological context of a situation is not automatically established uniformly for all participants. Even from the perspective of a single participant, sense-making may be multi-layered, heterogeneous, and opportunistic, using any clue at hand to reach a usable set of meanings and orientations to events. Gumperz (1982) has been particularly concerned with mismatches of contextual understandings, particularly as these mismatches are culturally patterned, so that we do not recognize that the person we are talking to is engaged in a very different situational drama than the one we imagine we are part of. As well, the conversation analytic notion of the floor (i.e., the group framing of the communicative circumstances) highlights the contention or negotiation that occurs to establish any one person's control of the turn and the temporary definition of the situation. The situational definition that momentarily holds the floor provides an opportunity space or participation frame for actions and meanings (Goodwin, 1984; Hanks, 1996).

New remarks not only add to and redirect the discussion, they reframe and affect the meanings for all that came before. As conversation analysts are fond of 
saying, meaning is created in the uptake, or how people respond to utterances. Thus meaning is what people take the meaning to be, which they then react to in their further utterances and actions (H. Sacks, 1995). In their perlocutionary force, as Searle might say, utterances get taken as specific kinds of acts, as things having been done that then populate the intertextual landscape for ensuing utterances (Bazerman, 1999a; Latour \& Woolgar, 1979). This emergent, retrospectively-established context of things having been said, acts having been felicitiously accomplished, provides an intertextual (Bazerman, 1993a ; Swales, 1990) equivalent of kairos (Bazerman, 1994c ; Miller, 1992).

What is relevantly noticed as part of the context-those things attended to-is also at play. References in discourse are indexical; that is, they indicate or point to something outside the utterance. Thus utterances rely on construal of elements of context (including the framing social contexts that define the footing) to establish their meaning. References even construct the relevant physical and social places within which the talk occurs by identifying what is salient in the ambient world and what are the boundaries that organize local space-what counts as here or there, inside or outside, us or them (Hanks, 1990). Even such luminous and linguistically marked objects as lighted exit signs vanish from view as we enter into the footing of the seminar which indexes other realities for our cognitive attention. The exit signs only reappear to attention if we are summoned to an emergency footing by an alarm or if our minds wander from the seminar, looking for any other possible mental stimulation no matter how accidental and trivial. Relative distance and time are noticeable as particularly plastic in situations, but indeed the whole world that is discursively held in imagination and reconstructed as the landscape of our action is constructed in the talk (Chafe, 1994). Thus what things are talked about, how they are brought to minds of the participant, in what aspect and with what evaluation and purpose are all part of the typification of the interaction and social space.

In face-to-face communication all this adds up to a co-construction of context, reality, and meaning system, using socially typified frames and culturally laden symbols that allow each participant to make sense of a potentially "sensible" projection of meaning and the realities within which those meanings take place. This co-construction is constantly evolving through interaction which makes relevant the sense-making of all the participants. People literally collaboratively perform the world they are making sense of, the world they attend to, the world they are acting within. The social and material worlds humans are aware of are constantly being remade in the changing uptakes, footings, floors, frames, and indexical references. It is within this evolving world that thought collectives emerge, working in characteristic thought styles (Fleck, 1979). 


\section{THE SENSE-MAKING DIFFICULTIES OF LITERATE INTERACTION}

This co-construction of a world to be attended to and made sense of also happens in literate communication across space and time, though it is faced with additional difficulties. In the semi-private experience of reading and writing, the clues writers offer to readers to reconstruct meanings are thinner than in face to face interaction. The referential space itself is a projection of the text as perceived by the reader. Without the shared here and now of faceto-face interaction, literate action must rely even more heavily on genre to conjure interactional space and define content expectations (which Bakhtin, 1981 identifies as the chronotope, as we will discuss in the next chapter), and on other more explicit identifiers of what objects of attention will appropriately be attended to and from what perspective.

In non-co-present writing we have to construct the virtual meeting space and then enact congruent meaning performances entirely out of shared social cloth. We may snip and re-stitch from several available social cloths, but never so much as to make the patchwork unrecognizable, for then we lose our way as writers and readers. We must create the recognizable footings and grab recognizable floors - otherwise the floor evaporates, just as much as if everyone leaves a meeting. The selves and acts we create are in constant dialogue with anticipated and actual uptakes. In writing, though, information on how audiences respond to our utterances is typically less frequent, in circumstances far from those of the original utterance, and more attenuated than in face to face talk. Similarly, our reperformances of others' meanings through reading are not easily corrected or focused by others; we have only continuing attention to the text to search for clues to meaning to adjust and refine our readings to align with the breadcrumb trail to meaning left by the author.

Further, in non-co-present reading and writing, ambiguity or uncertainty as to the place, purposes, and participants of social meeting may do strange things to our sense of anxiety. Engaged with texts in private, we may perceive ourselves removed from the social constraints and uncertainties of every day face-to-face interaction. Privacy may free us to explore meanings and sentiments that we are afraid might cast us beyond the pale of acceptable public identities and acceptable relations with others. In reading we can explore the taboo under a plain brown wrapper. On the other hand, the lack of immediately reassuring others may allow anxieties to numb our processes of meaning-making. In reading we become afraid of who might see our books or catch us entertaining controversial thoughts, and in writing we worry whether we can dare put our forming thoughts to paper lest potential readers condemn us for what we write. 
To some extent all writing puts us on the line, asking us to perform novel selves which may have unanticipated consequences. Writing then leaves our words open to readers' interpretations and reconstructions that we might not be happy with.

The production and reception of texts are caught in a tension. In writing and reading we have the space to define situations and activities as we would see them. Yet to make ourselves intelligible to others and to gain the wisdom of others, we have to discipline ourselves to using signs and making sense in socially intelligible ways. Through shared tools of sense-transmission we make our separate senses, and thus define sensible differences. But those differences in turn stretch limits of linguistic sharing.

\section{SOCIALIZATION INTO LITERATE WORLDS}

Literacy education aims to introduce students into culturally formed practices of making sense in and of texts. In schools children are taught particular tools of inscribing information, experiences, and thoughts in texts and gathering information and reconstructing ideas from texts. They are also introduced to forms of literary interpretation and engagement. Outside of school, widely available texts, puzzles, games and other artifacts depend on and reward specialized forms of sense-making and engagement, relying often on school literacy practices. Those who may be avid readers but not so well trained in disciplines of schooled literacy may make sense more idiosyncratically, though perhaps more interestingly. At times we all engage in creative non-standard readings in pursuit of our own meanings and motives, but we can be held to account for more normalized readings of the texts within particular social circumstances. When we haggle over the obligations a contract has imposed we are often forced to read a text together, with our divergent readings accountable to adjudication by the courts. When we proclaim on the basis of a news story that the latest notorious figure is guilty, a contentious friend may ask how we can possibly come to that conclusion from what we read.

Similarly, writing gains expressive force not by going down purely private subjective paths, but by gaining wider command of the culturally available resources and by deploying these resources to create recognizable circumstances and enactments. Again the undisciplined writer sometimes may make very interesting texts, but their texts may be idiosyncratic and hard for others to orient towards in meaningful or at least consistent ways, so uptake either evaporates or rapidly wanders far from the vectors of authorial impulse. Within some genres of texts, often literary or advertising, movement away from the 
socially recognizable into the personally desired is indeed encouraged, but in other genres projection of our own meanings and desires needs to be focused and contained if we are to make intelligible sense of each other's words.

To gain a sense of readers' meaning making, writers have regularly sought local readers and editors to respond to their writing. Modern writing pedagogy has emphasized feedback; rapid cycling of responses by teachers; teacher sensemaking roles extending beyond evaluation on purely formal grounds; peer response and evaluation; and writing for varied, real, local audiences. Writing pedagogy and writing practice have also developed procedures for reading one's own text so as to take the part of others, particularly in revision processes. Rhetorical analysis also provides tools for seeing one's verbal productions from the outside, as they might affect others. All these techniques deepen attention to the interactional reality of the text and the meanings evoked in the minds of the readers.

The difficulties of making texts that will bring to readers' minds meanings that the writer seeks to evoke highlight how meaning is a result of evoking and organizing attention within specific textual interactions. Knowledge, information, beliefs, or other contents not brought to mind do not enter the communicative transaction and co-construction of meaning. While the world may exist richly and robustly outside our acts of communication, only those parts of the world brought into the communicative act are part of the meaning evoked. Even though vocabularies may be collected in dictionaries, and reference books may document the findings of various specialties, they bear on our conversations only insofar as we are familiar with them and they are present in the moment of communication.

Knowledge is not absolute, but only what circulates. What distinguishes disciplines of knowledge are procedures for warranting claims, standards of comprehensiveness in attention to sources, and practices of evidence gathering. The communal expectations and procedures to hold parties accountable form a larger context of relevance and attention for every utterance. Insofar as a member of such a knowledge community does not remember or pay attention to something everyone in the field should know, he or she loses credibility and authority. If a historian forgets the established sequence of events in narrating a revolution, statements lose their sense and are discounted as meaningless. However, the historian may not be expected to pay attention to sociological findings on social movements. On the other hand, the sociologist's statements about the same revolution lose meaning and credibility if they are not attentive to relevant sociological theories and findings.

In these cases of disciplinary knowledge as in other cases, meaning arises, relies on, is evaluated, and is constrained within social processes. Meaning is 
evoked by utterances which carry out speech acts and establish social facts. Utterances in written language take their form in the produced and circulated texts, but they only gain their meaning and success in the transaction mediated by the text. Meaning arises, contingently and locally, as one person speaks to another through a thin line of words; the art of writing is to make this holographic magic happen across time and space through the fragility of words. In those written words we see a world represented. 



\section{CHAPTER 10}

\section{THE WORLD IN THE TEXT: INDEXED AND CREATED}

As discussed in the previous chapter, what is indexed (pointed toward) in the utterance identifies what is noticed, thought about, acted upon at the moment. What is indexed is the intersubjective content of the interaction, insofar as each participant is fully attentive, and accepting the range of attributions and interpretations that might be made as to what the words refer to or index. The things indexed are the social facts represented, relied on, reinforced, created, or reasoned about in the course of the utterance. Those things indexed are also interpreted, reacted to, evaluated, taken a stance towards, or integrated with other things on the recipient's mind in the course of reading. The material indexed and the connections made among indexed items are usually considered the content or substantive meaning of the text, and provide the usual answer to the question of what did the text say. But another way of asking that same question is to ask what is the world assembled or represented in the text, and what happens in that world. From a speech act point of view this asks us about what is contained in the locutionary act. But if we remember that the locutionary act is itself an act of representation with its own felicity conditions, particularly in Austin's view, we must also ask what is successfully or felicitously established as a social fact within the textual space. Depending on the communal or disciplinary expectations and epistemological procedures, these social facts may also be held accountable to organized experiences of the material world, and thus gain the status of scientific facts, legal facts, historical facts, and so on-reportable and consequential in each of those domains.

Of course, each individual reader will bring to bear an idiosyncratic collection of thoughts, associations, and experiences that may lead to seeing the signs in the text indexing somewhat different ideas, experiences, or objects than the writer had in mind. Readers thereby construct different meanings from the text or evaluate the meaning differently, but the individualistic readings they develop are socially consequential only if they are brought back into a social dialogue that negotiates a communal meaning or at least creates a focused contention over meaning. As discussed at the end of the last chapter and we will explore more deeply later in this chapter, professions, disciplines, belief communities, and other epistemic social groupings serve to align participants to the same set of beliefs, associations, experiences, texts, and other materials 
that form a relevant context for understanding and evaluating each new text. Participants can then be held accountable to communally shared understanding of texts they read and write.

\section{LOCUTIONARY ACTS, IDEATIONAL FUNCTIONS, CHRONOTOPES}

Looking at the represented meaning of the text through the locutionary acts creating social facts indexed in the text (which are then brought together through syntactic or reasoning processes within the text) bears similarities to two other projects of considering meaning represented in texts: Halliday's examination of the metafunctions of language and $\mathrm{M}$. Bakhtin's concept of the chronotope.

Halliday's Systemic Functional Linguistics (Halliday \& Matthiesen, 2004; see also Halliday \& Hasan, 1976; and Halliday \& Martin, 1993) offers an account of how language functions to express meanings through systemic choices available to the user. The more deeply we understand the meaning potentials of systems of language, the more precisely we are able to express meanings. Halliday identifies three large dimensions on which we create meaning-which he calls metafunctions: the ideational metafunction, the interpersonal metafunction, and the textual metafunction. As suggested by the names, the ideational metafunction refers to the means by which ideas or contents are transmitted, and thereby the way in which our experience of the world is represented and construed in the text; the interpersonal metafunction mediates the social relationship between speaker/ writer and listener/reader; and the textual metafunction indicates how a text is organized and serves communicative purposes. The ideational metafunction is closest to what I suggest by the text indexing experiences of the world and the textual metafunction is closest to how these indexed items are reasoned about. As a linguist, Halliday is most interested in the form this indication takes as it becomes represented in the text and then how syntactically these representations become organized into larger systems of cohesive reasoning-keeping in mind that the explicit linguistic markers of cohesion are distinct from the semantic psychological phenomena of coherence.

Bakhtin makes a specific association between genres and particular kinds of contents through his concept of the chronotope, or time-space. Within the typical time-space of each genre there appear typical settings, objects, and characters; each of these then undergo particular actions or events in the course of the text (Bakhtin, 1981). So just as fairy tales occur in kingdoms long ago 
and far away, where princes overcome obstacles of dragons and evil sorcerers to gain the hand of princesses, so do national economic policy reports include trends in jobs, Gross Domestic Product, national indebtedness, and interest rates, as well as projections of future growth and inflation, so as to justify policy decisions, such as adjustments of bank rates. Psychiatric reports prepared as part of sentencing of criminal defendants contain different chronotopes of information, looking into the time-space of the defendant's life, psyche, and prognoses under different incarceration conditions. We would be very surprised to find the information from the criminal psychiatric report in the economic policy document, or vice versa. Even closely related documents might differ greatly on their chronotopes based on the purpose, as the psychiatric sentencing document would contain different information from a psychiatric journal article on the pharmacological treatment of certain forms of violent behavior. Thus once we are attuned to a genre we are attuned to expect and accept indexes of different aspects of experience, to be represented and construed in certain ways, appropriate to the activity systems associated with those genres. The introduction of atypical contents into the genre requires extra work both to justify the contents' place and to translate those indexed contents into terms appropriate for the genre.

\section{GENRED ONTOLOGIES AND THE WORK OF EXPANDING THE WORLDVIEW OF THE GENRE}

Following Bakhtin we may note that each genre contains its typical landscapes, actors and events, which we can consider the genre's ontology. Each text also has its more specific ontology: that is, the objects that come under its purview. Thus in a newspaper editorial commenting on the actions of a chemical company, the chemicals that are part of the story (that have been determined to have harmful side effects, for example) may be referred to by common names or some abbreviation, but there would not likely be detailed chemical nomenclature nor analysis of the processes of synthesis. Chemical formulae and reasoning through a series of chemical processes would enter more typically into an article in a chemistry journal. If for some news-related reason the news story needed to discuss chemical processes (such as a discussion of how an apparently benign process has lethal consequences), the story would need to prepare and motivate readers for this excursion (Latour, 1987 gives a revealing analysis of rhetoric of detours) and then would need to ensure the specialized representation would be intelligible to them. 
Fleck, similarly, in his 1935 groundbreaking Genesis and Development of a Scientific Fact, analyzes the representational styles that constitute the thought styles of thought collectives. These representational styles are the means by which facts take on textual presence. He finds ideology, theoretical commitments, and evaluative stances in the various representational styles of the symptoms and underlying mechanisms of what we now call syphilis (Fleck, 1979). Indeed some objects are constituted only as they take on the form dictated by the genre and the text. The U.S. tax form, for example contains many objects that though they have names made of familiar words are only specifically constituted within the forms and the attendant regulationgoverned operations_such as "net reportable income," or "allowable deductions" (Bazerman, 2000b).

Conceptually-based objects that we talk about as real and tangible are only the construct of tangible operations. For example, while we can concretely observe money and goods exchanged between persons, the concept of an economy requires the aggregating of many transactions within a specified domain and reported to audiences ready to comprehend the concept. Even at the time of Adam Smith the modern concept of an economy was not available, and the closest term he could come up with was the wealth of nations. For most middle-class citizens the idea of the economy only became a familiar object of attention when it started gaining regular reporting in the newspapers, as something bearing on the conditions of everyday life (Smart, 2008). Indeed countries even into the twentieth century that lacked the textual means to collect, aggregate, and report on the economy only had individuals and families of wealth engaging in particular transactions and relationships. In order to become international economic players they had to gather those transactions and holdings into a picture of an economy, by establishing a ministry of the economy and producing economic reports, where the state of the economy could be found (De los Santos, 2007).

Thus we can associate each genre as a site for particular kinds of knowledge that we can expect to find there. We know where to look if we need a phone number, or government statistics on school completion rates, or latest medical studies - and if we don't, search engines will direct us to the kinds of webpages that contain what we are looking for, and we can use our genre knowledge to rapidly evaluate whether the site contains the kind of knowledge we want in the depth, reliability, and perspective we want. Further, we know where not to look for things or where we would be surprised to find information out of its genre place. One way, in fact, to trace the history and social distribution of knowledge is to trace the histories of genres in which knowledge is produced, reported, and collected (Bazerman \& Rogers, 2008 a \& b). 


\section{EPISTEMOLOGY, ACCOUNTABILITY, AND TRUST}

The chronotope or ontology of each genre and its appropriate forms of representation also imply an epistemology - a way of knowing. This way of knowing is associated with methods of observing and recording things, experiences, phenomena or the like, thereby indexing them in the textual world of the genres of a social world. Epistemological issues accompany even everyday description of ordinary events in our life. If we tell a story about what happened to us, unless we somehow mark it as a fiction or a joke, it is assumed that we experienced the events as we reported them-we tell what we saw and heard, from our perspective, drawing on our memories. Often we may signal as well the timing of the events as recent or long ago, commenting on the freshness of our memory, and identifying what we directly saw or found out only by hearsay. Listeners will interpret what we have said on that epistemic basis, giving it the authority of personal experience. While exaggerations are often accepted as part of emotional heightening, readers may detect these and factor it into the evaluation. If there is further indication of fictionalizing, the readers also begin to take the report as less reliable (with grains of salt, as we say informally). Even intimate reports of emotions are expected to come from recognition of actually experienced sentiments. Suspicion of violation of that epistemological procedure by reporting emotions never experienced, will likely have consequences for trust and evaluation of character. Listeners may, furthermore, recognize the possibility of different accounts from other perspectives, and they may factor in what they might know about our emotional set, evaluative biases, interests, or other personal elements that might define the particularity of our perspective.

Other kinds of reports also have their implied epistemologies. Accounting and business reports have their standards and practices of data gathering, authentication, and reporting-historically developed and often regulated by commercial law as well as professional licensing bodies. In the same vein, journalism over the past hundred and fifty years has developed professional standards for reporting, which are in essence epistemological guidelines for gathering, authenticating, and inscribing information.

Each epistemology implies a theory of the world and a related theory about observing and knowing the world. We have folk theories about how people experience emotions and how those emotions are triggered by events. Accounting principles are based on theories of accounting and how they keep track of business dealings, making them accountable and ordered through reporting practices; these in turn are built on theories of how accounting improves business practices and the economy. Legal rules of evidence also 
have epistemologies and practices that accept certain kinds of testimony and evidentiary documents as legitimate and legally meaningful and others as not.

\section{SCIENTIFIC EPISTEMOLOGIES, METHODS, AND VISIBLE PHENOMENA}

Over the last several centuries epistemological and methodological issues have been at the forefront in the sciences, as science has developed methods of observation and verification upon which to warrant claims and thereby to formulate knowledge through empirically-grounded argument. The emergence of modern forms of scientificargument has gone hand in hand with the emergence of definitions and standards of what counts as legitimate evidence and legitimate procedures of gathering that evidence. As a consequence, methodology has become a standard explicit feature of experimental reports, both to legitimate the evidence and to make it interpretable in relation to its procedures (Bazerman 1988, 1991). Some disciplines, such as experimental psychology, have explicitly regulated epistemologies and methodologies through the standards of reporting in their publication manual (Bazerman, 1987a). In all disciplines, articles are accountable for establishing the status of evidence and the methods used to produce it, including the theoretical assumptions behind the methodological choices. One of the most effective ways to undermine an experimental or observational report (and thereby undo it as a representational speech act) is to argue that there were faulty assumptions behind the methodological procedures or concrete errors in the material carrying out of the method, so that the data produced does not reliably represent the underlying state of affairs one is investigating. The most damaging criticism is to demonstrate the results were entirely an artifact of faulty method and there was no underlying phenomena of note thereby observed: nothing to be seen, nothing to be reported.

Further, each field has developed its particular methods and epistemologies (with corresponding genres of reporting) deemed appropriate to its objects of investigation (or ontologies). These methods and epistemologies in dialogue with the empirical experience of investigations produce the data reported and analyzed in the field's articles-thereby constituting the objects that come to be known and pondered by the field in its seminars, congresses, journals, and (eventually) textbooks. Even within biology, the methods, epistemologies, evidence, theories, and textbooks of botany differ from those of zoology; within botany differences occur among taxonomic botany, evolutionary botany, and genetic botany, although they at times have come to communicate with each other and rely on each other. But every cross-specialty communication requires 
some adjustment and negotiation about what constitutes knowledge, how it is to be produced, how it is to be represented, and what it means (see for example Bazerman \& de los Santos, 2005).

We can see this explicit concern with methodology and standards of evidence production as the realistic and practical consequence of the Baconian distrust of language and the early Royal Society injunction to trust things, not words (Dear, 1985). Yet in order to enter scientific discussion things still must be represented in words, mathematics, or other signs. Epistemology, methodology, standards of evidence production, along with the instruments used to produce, measure, and record phenomena (what Latour \& Woolgar, 1979, call inscription devices) negotiate the transformation from experience into inscription. Although Wilkinson and other seventeenth century enthusiasts may have hoped to expunge language of any uncertainty and thus only report true things (parodied by Swift in Book Three of Gulliver's Travels) —yet language and representation could not be done away with. One always needed to argue for the existence of phenomena and their interpretation. Even what could be seen by a telescope (Moss, 1993) or microscope (Ruestow, 1996) needed theoretical argument to legitimate the observations as data and needed theories of the workings of the instruments to interpret their results, tell true objects from evanescent artifacts, and refine methods. Advances in theories have been tied to advances in instruments, and advances in instruments have been tied to arguments warranting them and their validity as evidence producers. Further, the form of evidence each produces then enters into the expected and legitimate forms of representation in articles to then be considered. Even the relevance of mathematics within biological argument required explicit argument (Wynn, 2012).

Such arguments led to ever increasing standards for observation, to make phenomena visible and confirmable. Fleck characterized the ongoing search for more refined, more warrantable, more precise ways of seeing new dimensions of phenomena and making them reportable as an essential part of the culture of science. He called this the active pursuit of passive constraint-actively finding ways to be constrained by empirical experience in what one could say (Fleck, 1979, p. 95). Sciences are particularly persistent in their search for ways to produce more evidence of a more sophisticated type to test and advance reasoning and beliefs.

Sciences have developed regular practices of interrogating evidence, and confirming it against multiple experiences arising from multiple purposes—of which the well-known replication of experiments is only part. Sometimes high motivation, interests, and stakes spur direct replication attempts, especially when there is an astounding discovery claim, such as the announcement of cold fusion (Taubes, 1993). In the cold fusion case (as with N-rays a century 
before, Ashmore, 1993) other scientists could not replicate the results, and the phenomenon vanished from the literature, only to become an episode in the history of science (although now, more than two decades later a few scientists continue to search for confirming data). Most findings, however, are more ordinary and less unexpected. Many are of such detail and limited interest that no one questions them, and perhaps few even notice the article and fewer use it to any purpose. In a sense too these articles vanish from the canon of knowledgeexcept that their very ordinariness and consistency with expectations come to reconfirm all the previous findings, assumptions, and theoretical claims on which they are based. In that sense, the most ordinary and humdrum reports are indirect replications of much collected knowledge of the field.

At times, though, conditions cannot be replicated, or require craft knowledge for replication (Collins, 1985; Delamont \& Atkinson, 2001; Gilbert \& Mulkay, 1984) or few have the incentive, leisure, or resources to devote to replication. Yet when researchers carry out further investigations they must select and rely on the phenomena and evidence of others reported in the literature. When anomalies turn up in their results, they are then led back to examine earlier results that they relied on. The mention and use of these earlier findings as useful and reliable keep them alive in the intertext of the field and thus their representations stand as successful speech acts. Through continuing usefulness results enter into the canon of knowledge in a process of rolling codification (Bazerman, 1991).

\section{POINTING AT OTHER TEXTS: INTERTEXTUALITY}

This reliance on previous texts is part of the intertextuality that pervades the literate world. All utterances occur in the context of previous utterances, providing the resources of a common language, saturated by prior uses and beliefs. Further, new utterances take a stance towards prior utterances, respond to them, refer to them, and even incorporate them, as Volosinov noted (1973) and Bakhtin elaborated (1984a, 1986). As writing typically creates an enduring archive of prior documents which can be referred to, the relationships of utterances potentially become more complex, explicit, and robust-supporting systematic reliance of texts on each other, particularly in organized domains such as scriptural religions, law, academic disciplines, or corporate and governmental bureaucracies.

Among the objects brought into a text are other texts. A newspaper in reporting an unfolding scandal may refer to previous revelations reported in previous days' issues as well as quotations made by accusers, accused, and 
witnesses. The story may also refer to revelations reported in other publications and confirmed by still others. Then an editorial may reprise the aggregated facts from the various news stories to comment on the events and evaluate the roles of the various participants. Particularly embarrassing or self-condemning statements printed in other stories may also be reprised.

Each domain has its particular universe of genres and prior texts that are chronotopically relevant. Thus law cases typically refer to constitutions, statutes, prior cases, and court judgments considered relevant precedent, along with the various filings and briefs presented in the particular case. Within the relevant domain, the textual references may additionally be represented variously according to genre, as Devitt (1991) found out with respect to the genre set of tax accountants. While all the documents prepared by tax accountants rely on the tax code, the code gets quoted, mentioned, or implied in different ways, according to the kind of document, its audiences, and purposes.

Within sciences the creation of communal knowledge through the aggregation and building on work of the relevant disciplinary colleagues is associated with explicit and patterned practices for mentioning the relevant prior work to set the stage for each new piece of work (Bazerman, 1991; Swales 1990, 2004), as well as genres directed toward collecting, aggregating, and codifying knowledge both for insiders (Myers, 1991), and neophytes or outsiders (such as textbooks). Each discipline and journal also adopts explicit means of representing other texts in the form of citation.

\section{THE INTERTEXT AS A RESOURCE AND A CONTENDED TOPIC}

Intertextuality does more than become an indirect way to import the information reported elsewhere. Intertextuality can become a site of discussion, a domain of action, and a set of objects in itself. Sequences of documents may form the domain of a policy debate, where a cluster of related documents contend for which statement may become authorized as policy at the end of the discussion. These documents may be clearly structured as through the various filings, briefs, previous court transcripts, and rulings, defined by the rules of the court in an appellant court case (which in the US are carried out entirely by review of the file). At the end a judicial ruling sorts all the relationship and standing of all the documents in all future actions, subject to further appeals.

Political debates over issues of the moment are more loosely structured, and often lack the finality of a legal judgment so that disputes and differences are ongoing, always ready to be reprised, even after a quiescent period. But actors 
make claims and arguments, sometimes explicitly referring to and contending with, reevaluating or even mocking earlier statements, through what Bakhtin calls double-voicing (1984b) with the aim of changing the public's view of prior statements, and influencing what views should be left standing as effective persuasive acts. Sometimes the contentions of prior documents are recounted as personalized dramas of power, with the standing of statements going up or down depending on how punches are landed, and how they are reported.

Even claims within sciences can be seen from this view, as people propose claims that they believe should be considered for enduring presence in the disciplinary ontology or that will restructure or modify the epistemology or theory. Articles present evidence and interpretation, show value for a claim's continuing use, or advance alternative claims. In the end, some claims and concepts initially indicated by citation to articles (Small, 1978) have robust continuing presence in the ongoing investigations of a field, revealed in their citation rates (De Bellis, 2009). Ultimately, however, explicit citation may vanish by the implicit incorporation into the trusted knowledge accepted by all in the field, in a process sociologists of science have called obliteration by incorporation (Cozzens, 1985; Merton, 1973).

For those with an insider's understanding, any intertextual domain can reveal itself as a social drama, as proposals for a census or an accounting procedure may reflect the interests of different groups who imagine they would benefit from one method or another. Those in the know can track the changing fate of interests as the status of proposals rise and fall and some gain long-term incorporation into the accepted knowledge and thinking of a field. Through such contentions texts enter into the chronotope of a field, becoming part of the accepted and expected landscape of a particular genre embedded within the larger system of genres that comprise an activity system. Any variation from the chronotope, introducing unexpected intertextual landscapes, attracts notice and may require additional justifying or reconciling rhetorical work.

\section{INTERTEXTUALITY AND SOCIALLY- FORMED CONSCIOUSNESS}

Intertextuality occurs at the level of text, as one text relies explicitly or implicitly on another, but it has large sociological and psychological implications. Intertextuality provides mechanisms for forming communal beliefs and individual consciousnesses, even while fostering the possibility of focused division among individuals based on their selection and evaluation of texts and the way they incorporate those texts into consciousnesses and actions. 
A history of the theoretical elaboration of the concept of intertextuality makes evident the sociological and psychological importance of intertextuality among people who share universes of texts and activities. The term intertextuality, or any Russian equivalent, does not appear in the works of either Bakhtin or Volosinov. The term was first coined by Kristeva (1980) in a work of literary theory. Drawing on Volosinov and Bakhtin she suggests that any text is a mosaic of quotations. She uses the concept of the textual mosaic to argue against the radical originality of any text and to locate common cultural experience in the sharing of text rather than any shared intersubjective state, for we always take up individual subject positions. Orientation to common utterances, she argues, creates the ongoing culture and evokes common objects of desire. Intertextuality, for Kristeva, is a mechanism whereby we write ourselves into the social text, and thereby the social text writes us.

The origins of the concept in Bakhtin and Volosinov have different motives and forces than used by Kristeva. Volosinov (1929/1973) notes that every utterance draws on the history of language use, is responsive to prior utterances, and carries forward that history. In the interplay with past utterances, each new utterance takes on a stance toward previous utterances. Volosinov, furthermore, begins a technical analysis of how texts position themselves to each other through linguistic systems of direct and indirect quotations. Since Volosinov sees individual consciousness arising out of our particular experiences of language utterance, our consciousnesses are deeply dialogic (or as we would now say intertextual), just as our utterances are. Therefore the mechanisms of textual relations are also part of the mechanisms of the formation of consciousness (pp.12-13). Volosinov's comments on the internal formation of consciousness through dialogic experience of language are close to issues raised by Vygotsky's analysis of the internalization, as Vygotsky explains in a 1931 essay on the internalization of higher mental functions:

An interpersonal process is transformed into an intrapersonal one. Every function in the child's cultural development appears twice: first on the social level, and later, on the individual level; first between people (interpsychological), and then inside the child (intrapsychological). (Vygotsky, 1978, p. 57)

Volosinov in his 1927 book Freudianism (1987) already was concerned with the issue of inner speech. In this context (p. 21) he cites Vygotsky's 1925 paper on consciousness as a core problem of psychology, where Vygotsky begins his investigation into the way language mediates consciousness and transforms 
reflexes, thus making available for consciousness and thought a form of cultural transmission of the historical experience of humankind, as we have examined in Chapter 2 of this volume. These ideas, however, were only sketchily gestured at in the 1925 paper. While Volosinov's 1927 citation provides direct evidence of Volosinov's awareness of Vygotsky, it is also reasonable to assume that Vygotsky was aware of Volosinov—given Vygotsky's extensive reading, the close world of Soviet science at the time, and the consonance of their interests in developing Marxist historical theories of the formation of language, the mind, and consciousness.

Vygotsky's ultimate formulation of an internal plane of consciousness resulting from the internalization of language experience would provide a more robust model of socially-formed individual consciousness and agency than Volosinov's formulation of inner speech and consciousness. Vygotsky, as a psychologist with developmental interests, was looking at how the outside (the interpersonal) got inside (the intrapersonal) in order to shape individual thought and action. He thus elaborated mechanisms by which internalized thought operated within the functional system of the self. The internal plane of consciousness, formed when language experience integrates with nonlinguistic experience, incorporates one's earliest social and linguistic relations and reformulates one's prelinguistic and non-social experience and perception.

If Vygotsky shows more fully how society gets into the self, Volosinov as a socially-oriented linguist points outward into how the self gets into society. Volosinov's formulation of inner speech arising out of socially embedded utterance reaches further outward in planting individual consciousness within a dynamic and complex social field. He points to the linguistic mechanisms by which we become intertwined with others in social dialogue and by which we necessarily become reliant on others' words in talking with and interacting among people. Because his work as a linguistic theorist and researcher did not extend much beyond his 1929 book, he never developed further his investigation of the socio-linguistic mechanisms of the embedding of the self in social relations and utterances. His work, nonetheless, has set important terms for contemporary sociolinguistics and anthropological linguistics. The strong complementarity between Vygotsky's inward mechanisms of the sociallyformed language-saturated consciousness and Volosinov's outward mechanisms of consciousness-forming socio-linguistic utterances provide a meeting point between psychology and social studies of language and interaction.

The dialogic formation of consciousness is a theme pursued by Bakhtin (1981), in particular concerning the representation of novelists' consciousness expressed through the utterances of the novel's characters and narrators. In Problems of Dostoevsky's Poetics (1984a), a reworking of a 1929 book on 
Dostoevsky, and The Dialogic Imagination (1981), representing work in the 1930 s and 40s, he associates the form of the novel with a form of consciousness. He praises novels that recognize the variety of utterances incorporated and thus adopt a stance of multivocality, dialogism, or polyphony rather than authoritative univocality, monologism, or monophony, which obscures the complexity of human language, consciousness, and relation. Bakhtin's interest is in valuing appreciation of the existence of others, in the neo-Kantian tradition familiar to us in such moral thinkers as Martin Buber (1937) and Carl Rogers (1961). Bakhtin's moral stance starts with a morally accountable, autonomous self that must take responsibility for individual actions, as he articulates in his early works published in Art and Answerability (1990) and Toward a Philosophy of the Act (1993). Such an individual moral self implies a very different form of consciousness than that from internalization of socially embedded speech presented by Volosinov and Vygotsky. For Bakhtin dialogism is a moral imperative as well as a fact of social development, that we draw on the pre-existing world of utterances to provide the resources for us to form our own utterance.

\section{INTERTEXTUALITY AND INDIVIDUATION}

Yet while Bakhtin explores forms of consciousness that tie one viewpoint with another, he also identifies mechanisms by which a writer distinguishes his or her voice from that conveyed in the other voices incorporated into a complex consciousness. Bakhtin, in works such as The Dialogic Imagination (1981) and Rabelais and his World (1984b), considers stance, attitude, and evaluation one utterance makes toward others, such as through double-voicing or carnivalesque. He particularly considers parodic or otherwise critical heteroglossia as forms of resisting or commenting on authority, power, and dominant classes. His treatment of double-voicing highlights the complex attitudes we have towards each other's words as we recognize and reevaluate the character of each other's voice. Such complexity of evaluative attitude can serve to exclude or depreciate the other. To keep those who are different from us at a distance, we might parody a foreign accent or non-dominant dialect or we might mockingly repeat words we dismiss as absurd. Bakhtin, however, attempts to maintain a democratic, neo-Kantian appreciation of the other by limiting the targets of what we would now call attitude. The examples of carnivalesque or linguistic mockery that he examines typically aim to deflate oppressively powerful ruling forces rather than to stigmatize the powerless.

Bakhtin provides conceptual tools for understanding how authors engage or repress complexity of perspectives and establish attitude towards the perspectives 
of the characters they represent. He uses those tools to analyze in detail how the interplay of voices and perspectives is managed in different texts with particular ideological implications. In a number of works he presents histories of different forms of consciousness associated with differing literary forms and the political struggles embodied in the replacement of one literary form by another. Later literary critics such as Kristeva, Barthes, and Riffaterre put aside analysis of the authorial handling of multiple voices and the historically shifting forms of fiction and literary consciousness to engage broad, ahistorical questions of the status of the author, originality, and interpretation. Kristeva (1980) coined the term intertextuality to dissolve the autonomous integrity of both author and reader into the ocean of shared cultural experiences of common texts. Barthes (1977) took the implications of intertextuality a step beyond Kristeva's dissolution of authorship to destabilize the text itself, since the text rests on the evocation of so many other texts. Riffatere (1984) sought to establish a basis for textual meaning and interpretation within the linguistic ambience, or intertexts, within which a text is read. Genette, however, has returned to a concrete analysis of how intertextuality works within specific texts. In several publications he has mapped out orderly sets of possible relations among texts, what he calls transtextuality (the making of meaning in an ambient world of texts), intertextuality (explicit quotation or allusion), paratextuality (the relation to directly surrounding texts, such as prefaces, interviews, publicity, reviews), metatextuality (a commentary relation), hypertextuality (the play of one text off of familiarity with another), and architextuality (the generic expectations in relation to other similar texts) (Genette, 1992, 1997a, 1997b).

Volosinov recognized that, as linguistic creatures, humans are inevitably caught up in the social drama of unfolding webs of utterances, to which we add only our next turn; Bakhtin then drew attention to the stance we take towards prior utterances. How we position ourselves against prior texts sets the terms for what we are able to do in the next step of the dialog. Volosinov's and Bakhtin's understanding of language as historically situated utterance opens up many issues of the way writing is situated within, deploys, and re-represents the flow of prior texts, but it is up to composition and rhetoric to articulate the complex skills and knowledge by which we manage to articulate our position and contribution to that intertextual space. If we are to understand how we are acted upon, how we can re-act, and how we can act freshly in this complex literate world of ours, where major institutions and spheres of activity are saturated by texts, we need to move toward a richer and more participatory understanding of intertextuality.

Intertextuality is ultimately about agency within the complex, historically evolved, and continuingly mutating landscape of texts. Even while a marine 
biologist must embed his or her contributions in the collected knowledge, methods, theories, projects, and motives of the field, he or she must offer a novel contribution which changes the intellectual landscape and reconfigures knowledge. The new textual landmark creates a new point from which to view a prior work in the field-a new perspective, a new evaluation, no matter how small or great in novelty. Likewise, a lawyer's brief must be embedded in and speak to the relevant archive of the law and the courts as well as the documents, evidence, and testimony of the case at hand; yet each new statement must somehow add to the client's case, with the intent of influencing the evaluation of all that came before in order to affect the final disposition of the case. Each contribution to a field of science or each successful intervention in a legal case changes the knowledge, precedent, beliefs, and ideas that are available for use and may be deemed relevant to be attended to by future participants, thereby changing the indexable resources and the playing field of future action.

\section{REASONING AND THEORY}

Intertextual references do more than indicate objects and statements from elsewhere. The various indexed ontologies and intertexts are brought together, placed in relation to each other, and organized to create a bigger picture or tell a story or make claim. Each text carries out some reasoning about its contents, even if just to list items in proximity and sequence. Further the patterns of representation, reasoning, ideas, or cluster of associations of each text stand in relation to larger structures of thought and belief that circulate in the domain the text is part of — what we might call theory or ideology.

The elements typical to a genre are not just brought into a space, but are put into relations and then interactions typical of the space. A news story brings together sets of characters familiar to readers because of their prominence in business, government, entertainment, or other domain or because they have been caught up in events considered newsworthy. But then the news story identifies particular relations among the characters: one has talked to another or made a deal or has been accused by someone else. We also expect to be told of journalistic attempts to get comments and responses by related parties. The reported events additionally are played out against larger frameworks of action — such as piece of legislation being negotiated over a period of time, or a history of suspected corruption, or a series of government reports about a series of problems, or the drama of the rise and fall of celebrities - all of these are the themes of numerous previous stories. 
The genres of academic disciplines equally tell stories of the advance of knowledge hoping to enlist readers into their view of events and accept the appearance of the new claim or theory that is the point of the article. These stories often begin by selecting from the generic chronotope of the disciplinary ontology of objects and problems and creating a selective intertext of what has been previously known - to set up the terms of an heroic adventure as Latour (1987) calls it or establish a niche for a claim as Swales (1990) calls it. To fulfill the heroic challenge or fill the niche, the researcher is presented as doing something new-framing a theory, carrying out an experiment, observing an event, performing an analysis-which carries the adventure forward and attempts to change the disciplinary landscape of knowledge. Of course there are many genres within each specialty with constant variations, but each attempts to move the disciplinary discussion forward by adding new items to the ontology or by rearranging perspectives and relations among prior statements.

In all disciplinary, professional, public, and other domains, larger activities of the field are carried out by more detailed arrangements within each text, walking readers down a path from one item to another with connectives to form logical or other persuasive relations. As the story unfolds, the sequence of events and the relations the article puts them into evoke judgments from the readers. When one government official is reported as being charged with payoffs, another is quoted as asking "who else has been picked up?" the readers' views of both parties and the events reported are confirmed or transformed. As details about the scope of an earthquake and the extent of the devastation are described in a story, readers come to evaluate the size of the tragedy. Then when told of the actions or inaction of various relief and government agencies the readers evaluate the adequacy of the response and are reassured or enraged. When told the stories of individual pain and endurance, the readers then view the events through different emotional coloring.

The writer tries to guide the readers' judgments by evoking values and evaluations at appropriate points, directing attention to certain kinds of evidence and phenomena, framing the story within particular ideas, reminding readers of earlier stories and events. The writer may also attempt to head off objections or alternative positions readers might hold, to answer possible questions about methods, to show distinctions between this and other cases, to remind readers of the importance of a distinction or to keep the readers from dismissing some part as tedious or trivial. In short, the writer attempts to keep ahold of the readers' modes of reasoning, calling to attention all needed to maintain and advance the argument and to exclude what might distract the readers from staying within the desired path of calculation. In classical rhetoric, this concern for sequence of thought would fall under the canon of arrangement, which 
at times was understood as setting out a psychological path to move people's minds and hearts, or as Bacon (1605) puts it in the Advancement of Learning, "The duty and office of rhetoric is to apply reason to imagination for the better moving of the will."

\section{TRUST AND PRIOR BELIEF}

Because the writer seeks to have readers give their minds over to the path of reasoning the writer sets out, establishing and maintaining the readers' trust is essential. Activating the readers' minds, filling them with the contents suggested, avoiding contents that would weaken engagement with the projected meaning, and following the path guided by the writer requires the readers to believe in the good will, honesty, and intentions of the writer. Otherwise the text can evoke a resistant reading, creating a counter-meaning against the text rather than recreating the meaning offered within the text. The moment readers find something wrong or objectionable or suspicious, they start to distance their minds from the text; mental construction of meaning becomes conflicted or even oppositional. The larger the causes for skepticism, the more readers' minds veer from the place the writer wishes. On the other hand, as long as the writer is able to bring readers along a shared path they enjoy a sense of consubstantiality as Kenneth Burke (1950) called it, drawing on the language of religious communion. With readers sensing a shared substance with the writers, readers identify with the meaning, projects, and even subjectivity of the writer. The reader attaches his or her own motives, associations, and meanings to the words of the writer, who is felt to be a kindred spirit. Other satisfactory relations of more limited trust are also available; for example, readers remain cooperative or at least compliant with bureaucratic communications as long as they sense that the bureaucracy is acting properly and believe that compliance serves their interests.

Using the generally accepted theory of a shared domain elicits trust of readers holding those same theories and decreases the work of establishing a common basis for reasoning. If the overwhelming majority of newspaper-reading citizens hold the view that wars are to be understood as personal contests between leaders and the value of their cause can be measured by the morality of the leader, then such beliefs can be invoked in the reporting of government justifications for attacks and of the deeds of leaders as virtuous or immoral. Every time this theory of war is invoked, explicitly or implicitly, it becomes more firmly entrenched as a warrantable form of reasoning in the genres invoking them. If, however, people see war in terms of the costs to citizens, accounts of the conflict 
to be trustworthy and credible must focus more on the lives of people caught up in the events and their attitudes toward the conflict. If readers hold the view that war is a strategic intervention in long-term geopolitics, then texts must tell an entirely different kind of story to be perceived as relevant and credible (and not just an untrustworthy account of either jingoistic war-mongers or bleeding hearts, as such readers might stigmatize accounts from other perspectives).

This relation between theory and what is perceived as a trustworthy account is equally the case in the sciences as it is in the public sphere-although processes that establish trustworthiness may differ significantly. As a scientific theory becomes established and warranted, with decreasing questioning and challenge, it becomes an unquestioned resource for reasoning in the field. In the first half of the twentieth century, for example, quantum theory became accepted and embedded in small particle physics, so that it became ever more implicitly built into the structure of reasoning and arrangement of the article, and thus part of the generic expectation (Bazerman, 1984a). The process of its becoming accepted and regularly invoked was tied up with evidence, accountability, and an emerging intertextual web of confirming studies, so that the theory became a trusted and reliable resource for the field. A part of such a story is how the physicist Arthur H. Compton argued over a series of articles that particular new forms of evidence about observed phenomena were best explained by quantum theory, supporting a larger movement in subatomic physics from classical to quantum theory (Bazerman, 1984b). As questions were stilled, researchers found quantum theory a useful resource to be regularly invoked in ways that would not raise questions about their own work, but rather would support their credibility. The theory that was once considered speculative and suspect became taken for granted, invoked with regularity, and with decreasing amounts of work needed to warrant it. A paper that did not then rely on the theory, overlooking what any insider would see as obvious quantum effects, would then become suspect and less trustworthy.

Aristotle (1991) called such beliefs held by a community that are usable without explicit reasoning as enthymemes. Audiences are especially attached to messages that invoke enthymemes they hold, because the enthymemes tap unarticulated beliefs and match their own judgments. Using the implied reasoning of the enthymemes, they come to conclusions that match the rhetor's without coercion or urging. They sense that the rhetor thinks like them and is therefore even more to be trusted. This goes as much for racist diatribes against immigrants as for hortatory sermons inspiring virtuous actions as well as for scientific reasoning relying on shared knowledge of the field. The degree that these assumptions when questioned can be made explicit and re-examined on the base of evidence and reason within the terms of the domain, however, varies 
from domain to domain. As with most of the textual phenomena discussed here, enthymemes are genre and field specific. The same audience that would accept the racist anti-immigrant rant, when reading recipes or restaurant reviews might entertain entirely different theories about the works and cultures of these same populations that they excoriate in political contexts. While research articles in particle physics would take quantum theory as a presupposition and prerequisite for trustworthiness, research articles in psychology would not invoke that theory, and its relevance to the reasoning of an article would take lots of explicit justification not to be viewed as crackpot.

Certain genres and domains of communicative practice explicitly attempt to make visible and call into question presuppositions of other genres and domains, so as to bring them to the surface for inspection, re-evaluation, criticism, change, mockery, or humor. If such questioning is successful, the reasoning in the questioned field can change, with different statements trusted and different assumptions evoked in shared thought. Ideological critical analysis attempts to surface unspoken assumptions of cultures, often to reveal inequities or power relations embedded in cultural practices, and thereby to make these practices less palatable or trustworthy. Comedians, in mocking the statements of others that are trusted by some audiences, point toward contradictions of assumptions or outrageous implications of cultural assumptions. A public figure successfully mocked by comedians, whether with political intent or not, has to contend with the changed public view and must work to rebuild lost trust. Public campaigns to change views on such policy issues as health, drugs, energy, environment, or diversity also aim to change the underlying structure of assumptions about which statements are to be taken as trustworthy and untrustworthy as people reason about their life choices. But those who wish to question assumptions in any domain, for whatever reason, must themselves earn trust among those whose presuppositions and reasoning they wish to change. Cultural critics can be dismissed as uninformed malcontents; comedians who transgress too far can be viewed as nasty rather than funny; and public campaigns to change belief can themselves be the object of mockery and disbelief.

\title{
THE INSUBSTANTIAL PAGEANTS OF MEANING
}

\author{
Language is realized in the form of individual concrete \\ utterances (oral and written) by participants in the various \\ areas of human activity. These utterances reflect the specific \\ conditions and goals of each such area not only through \\ their content (thematic) and linguistic style, that is the
}


selection of lexical, phraseological, and grammatical resources of the language, but above all through their compositional structure. All three of these aspects-thematic content, style, and compositional structure-are inseparably linked to the whole of the utterance and are equally determined by the specific nature of the particular sphere of communication. Each separate utterance is individual, of course, but each sphere in which language is used develops its own relatively stable types of these utterances. These we may call speech genres. The wealth and diversity of speech genres are boundless because the various possibilities of human activity are inexhaustible, and because each sphere of activity contains an entire repertoire of speech genres that differentiate and grow as the particular sphere develops and becomes more complex. Special emphasis should be placed on the extreme heterogeneity of speech genres (oral and written). (Bakhtin, 1986, p. 60)

The practice-based approach to genre we present here has synthesized multiple lines of influence: 1) developmental theories of self and consciousness arising in social interaction saturated with language in order for social creatures to seek life needs and satisfactions; 2) phenomenological sociology, which finds the emergent order of everyday social activity resting on processes of typification and recognizability; 3) pragmatic theories of self and society, seeing self, society, institutions, language, and meaning constantly being transformed to meet human needs; 4) structurational sociology, which sees larger structuring of events and relations emerging interactionally from the local actions and attributions of participants; 5) anthropological and psychological studies of discourse practices as situated, distributed, and mediated; 6) speech act theory, which sees utterances going beyond conveying meaning to making things happen in the social world; 7) theories of discourse as dialogic, situated, and heteroglossic; and 8) a rhetoric oriented to content, purpose, and situation as well as form and style.

This synthesis leaves us with a view of text content and meaning as transient and unstable, a construct of readers in dialog with the signs inscribed within the text. The construction of meaning, however, is not randomly idiosyncratic, but rather relies on participants' positioning within activity systems, social groupings, larger cultures, personal histories, and immediate motives. Texts point towards various objects in the world and collections of prior discourse, and invoke procedures of construal and communal understanding, so as to 
agree on what is being pointed to-or at least well enough for participants to continue communicating without a breach of trust.

Further, texts attempt to enlist participants into communities of shared knowledge, thinking and activity-so that the text becomes an object of co-orientation and shared knowledge. Texts become vehicles for forging intersubjectivity, even as there is a projective variation in the meaning each reader attributes to the text and to what is being indexed by the words in the text. The degree that texts are able to evoke the degree of co-orientation and coordination of meaning and action that they do in particular spheres is remarkable since the coordination of meaning rests on the transient phantasms of people's minds - the passing dramas played out on the neural projections of individuals' brains. But of course, each text is surrounded by complex social, historical, and cultural apparatuses that bring people together in common projects and experiences, that have made them familiar with what is pointed to in each text, and have facilitated shared attitudes towards those things indexed.

If sharing of meaning is a function of social, cultural, and historical propinquity, then the sharing of meaning becomes more difficult the further the reader and writer are separated by domain, period, region, project, or viewpoint. Writing any but the simplest and most familiar meanings to one's closest peers is difficult. The further apart the writer and reader and the more complex and unanticipated the message, the more gets lost to the accommodation of meaning between worlds. Texts that are clear, strong, travel, and carry more than the most conventional meanings deserve admiration. 



\section{CHAPTER 11}

\section{THE WRITER ON THE SPOT AND ON THE LINE}

Forty years ago when I began to inquire into what kind of writing students needed to be able to produce to succeed in their academic endeavors, I was drawn into a vortex of individual and disciplinary differences. I was led from texts to disciplinary endeavors, to intertextual relations, to histories and transformations of genres, to theories of society, action, consciousness, and cognitive development. In this volume I have tried to lay out how these various theories can fit together into a picture of individuals situated in specific historical and social circumstances acting through writing and participating in the social life unfolding around them. Much of my empirical work has been about historical emergence of literate forms, the knowledge expressed within them, and individuals acting within specific circumstances. In many of these studies theory has been developed in pieces, and in relation to the issues raised by each case. To complete the picture, this volume draws those various pieces of theory together in what I hope is a coherent and persuasive account.

This volume provides an account of the local production of purposeful meaning within textual interaction, and an account of genres that facilitates the alignment of people in their communicative interactions, particularly over texts. But in so doing, this volume has also proposed processes by which meanings and the conditions of complex meaning-making spread over larger groups engaged in activity, and how these groupings and their opportunities for meaning-making evolve. That is, literacy facilitates communicative interaction among expanding groups of people, and document-mediated relations facilitate wide-spread meanings and knowledge, forms of extended social organization, and the rise of institutions. This account does not rely on abstract, out of time conceptions of language, society, knowledge, mind, or thought, but rather proposes concrete processes of communicative action among individuals building the larger structures of modern distantiated society on an expanding collection of small inventions of language, technologies, textual representation, social and material relations, and literate practices. The ideas here position the writing self within historical circumstances to unpack the psychological complexity of someone attempting to produce effective texts for his or her circumstances and developing into a competent writer adequate to the opportunities and demands of the time. 
With the aid of this theory, I return, on the other side of the vortex, to the complex history and social arrangements that situate each person learning to become a writer today and which have formed the contemporary literate world. Much of this chapter will rest on summary reviews of prior historical and social inquiries. Rather than attempting to present that work and the related arguments in full, I refer you to other sources, much of which is gathered in the first two sections of the Handbook of Research on Writing (Bazerman, 2008), specifically devoted to "Writing and History" and "Writing and Society." I close with some comments on the challenges a developing writer must face, based on the view of writing presented in this and the accompanying volume, $A$ Rhetoric of Literate Action.

\section{THE PROBLEMS OF SPREAD OF SHARED UNDERSTANDINGS AND ACTION}

Unless we are to posit invisible and abstract cognitive structures that act above the level of the human or deep within each organism through a preprogrammed human genome (and not indicated in any of creatures we evolved from), it is hard to account for the large structures of human language and activity that we participate in, extending over the globe and indeed now reaching out into the universe. While other animals communicate knowledge of food sources, threats, and even modes of play through chemistry, sounds, and visible behavior (and perhaps even limited symbols), they do not make that knowledge available to those of their species not in their immediate group nor do they develop large bureaucracies of record keepers and scholars whose work it is to produce, collect, and synthesize what the species or even the local cluster knows. Further, while other animals may pass limited information from generation to generation or group to group, they do not develop large structured organizations dependent on conscious regulation, information, and long cultural educations. Even humans prior to writing lived overwhelmingly local lives, orienting to the immediate physical surroundings and the social groups they saw daily-extended in space by the limited oral reports they would get about the past through the traditional lore and wisdom of their local group, distant realms that others claimed to have visited, and the material circulation of goods and artifacts through trade, plunder, and inheritance. Language facilitated the creation of local cultures and societies, but literacy made possible the large structures of modernity that distribute knowledge, orientation, and activities across greater distances than one can imagine, fostering both greater complexity of behavior and greater coordination that supports that complexity. 
Each face-to-face social encounter is local, gaining the attention of only those present. Each spoken utterance is local and enables coordination only among the interlocutors. Only enduring artifacts can create regularities and organization over time. A chance physical environment such as a cliff with caves, light, proximity to food sources, and a stable climate can create some continuity among life in an ecosystem over years, but if the physical system were to change, so would the life of all the creatures. Humans (even before literacy), more than other creatures, have organized their environments to create comfortable and continuing ways of life. In preliterate and prehistoric times, humans even created physical environments to embody knowledge of the heavens and the seasons on earth that would make agriculture and other living conditions more predictable. But you have to be there, living in the village at the Stonehenge or in the aural community surrounding it, to benefit from its organizing knowledge.

\section{LITERACY AND THE ORGANIZATION OF SOCIETY}

The development of modern society needed some further mechanism to create an organization that brought the local into larger regimes of organization and also brought the benefits of knowledge gained from many locales, coordinated, evaluated, and selected from. The marking of signs on stones, clay, paper, and now digital memories - each more portable and rapidly travelling than the previous_-provided means for increasingly coordinated and extended action as well as memory across larger groups of people over time and space. As Goody (1986) discusses in The Logic of Writing and the Organization of Society, writing changes the possibilities of the basic institutions of society: economy, religion/belief, law and government. None of these transformations are compulsory or automatic, and they play out differently in different societies and cultures, under differing geographic conditions and dynamics of culture and invention. Nonetheless, literate inventions facilitated greater wealth, business, and governmental power over greater distances; greater uniformity and predictability of laws over extended domains embodying concepts of equality of treatment; communities of belief defined by commitment to sacred texts as well as open to schisms over the meaning of texts; emergence of literate elites who controlled the knowledge gathered through literacy or scribal castes who worked in the service of other elites; and many other potential consequences which we can recognize in modern institutional life. Tiersma (1999, 2008, 2010) has examined the technical legal consequences of textualization of the law. Smart $(1993,2003,2006,2008)$ has considered the role of texts in the 
activity of financial institutions as well as the development of the economy as a system of textual transactions and records, and the very concept of an economy. Dorothy Smith has studied sociologically the formation and consequences of a documentary society with bureaucratic practices to regulate, monitor, and serve the life of its citizens (1990, 2002; Smith \& Schryer, 2008). Other social and cultural systems that have developed on the infrastructure of writing include journalism and news (Conboy, 2008), medicine and health (Schryer, 1994, 2002; Schryer et al., 2002), systems of professional work (Beaufort, 2008), commerce and corporations, and literary arts and entertainments (Hogan, 2008). Even our understanding of personal relations, romance, mental health, and spirituality have been deeply influenced and reorganized by forms of written communication, the literate circulation of beliefs and self-help practices, written reflective accounts of the self, and published scientific studies.

Literacy has also facilitated and become the medium for the production, distribution, and application of knowledge along with the associated institutions of libraries, education, academic disciplines, and research. Most of what we consider knowledge has been produced and is accessible in the form of written documents. As discussed in chapter ten, knowledge can be considered as the produced contents of texts, circulating within particular social networks through appropriate genres. The work of the institutions of knowledge is largely mediated through the production and circulation of texts, whether within the classroom or among colleagues. For a detailed history of the interrelation of the institutions of knowledge production and transmission, the associated genres, and the forms of knowledge valued and produced in various societies, see Bazerman \& Rogers (2008a, 2008b) and Anderson (2008). The medieval invention of the university, the early modern development of the genres and institutions of science (Atkinson, 1999; Bazerman, 1988, 1991; Gross et al., 2002) and the development and democratic spread of schooling (Olson, 2008) are particularly important for the formation of modern knowledge and information society.

Historically early scribal schooling taught the basic skills of reading and writing in tandem, as the scribes were the recorders, record keepers, record readers, and record users. However, as the archive of inscribed knowledge expanded, and certain productions became privileged (to the point of sacred texts being treated as having divine attribution), the maintenance and reading of texts became more widely spread and authorship more highly valued and restricted. Particularly in religious schooling, reading and interpreting of received texts took precedence over writing, placing the students subordinate to a received tradition rather than as co-creators of an ongoing culture of knowledge. The first priority in learning was to be aware of the texts that 
ruled life, and only with experience and selection would some be brought into interactive roles in producing the tradition. Most modern education is based on the principle that students need to know and attend to the knowledge gathered in the books, usually textbooks. Specialized school textbooks are designed for the transmission, explanation, manipulation, and application of knowledge valued by a society. In contemporary primary, secondary, and even higher education much student writing is reproductive reporting of material in textbooks and other assigned reading. Students are enculturated within a large sea of received knowledge, which they become accountable for to succeed in schooling and presumably in life afterwards.

Yet it takes positive acts of assertion to mobilize that knowledge for our benefit and interests and concerns. This is much of the dilemma of contemporary schooling - there is too much to transmit and become accountable for, so much that the time and opportunity for learning the positive skills of assertion may be pushed to the side. Without those skills of articulating and asserting our concerns and acting on them, however, we are buried under the weight of received learning, becoming the epigone that Nietszche warned us of in The Birth of Tragedy (1872/2008).

\section{THE CHALLENGES OF LEARNING TO WRITE}

Young people learning to write in contemporary society are, therefore, faced with many daunting tasks that extend far beyond the issues of transcribing letters, spelling words, and forming sentences that follow the prescriptive grammars. They must become familiar with the world of knowledge arrayed in existing texts and they must find how to represent that knowledge, respect it, use it for their own purposes, and perhaps have something to say about it. This means students have to spend many years becoming familiar with the received knowledge in textbooks, reference works, and other assigned readings. Much of their writing serves the learning function of familiarization with received knowledge and demonstrating that familiarity. It is then a further challenge to learn to think with and about that information, to have something to say about it that is not just repetition-whether that added value is in rephrasing, synthesis, reflective comment, personal association, critique, or new assertion or claim. While in the early years of schooling, students have some authority or authorship rights over their own experiences, feelings, or imaginations, and are able to engage in tasks local to their life-world, it is only at the more advanced levels of education and academic life that they gain gradual degrees of authority to substantively comment on, contest, or add to the body of received knowledge 
in most areas. For those students that endure that long in the academic world, moving from the role of knowledge receiver to knowledge maker requires many fundamental changes of stance and role.

A second level of challenge has to do with becoming familiar with and adept in the large number of genres and linguistic inventions that are available to write in-selecting among them and performing them competently (or potentially highly effectively) in relation to their purposes, form, organization, contents, appropriate lexis and register, stance, tone, and politeness conventions. Furthermore, many of these genres are lengthy and complex, requiring gathering and organizing of extensive material and thoughts into coherent statements with an internal logic appropriate to the genre. In the early days of writing, when scribes mostly wrote lists - tax rolls, inventories of property, chronicle lists of events, genealogies - the options for text organization were limited and highly determined by the tasks. The contents were similarly determined, though some care might be needed in collecting the information to be transcribed. Learning the techniques of transcription, the limited relevant vocabularies and their symbols, and the format of lists were fairly contained tasks; once one had mastered them one could write competently for that world. But as writing came to include such tasks as creating poetic narratives of the works of great kings or drafting wise laws that would be understood unambiguously across wide domains, then issues of extended composition and logic, alternative forms of expression, logical and associative sequencing of events and thoughts, internal consistency of heterogeneous statements, audience awareness, rhetorical effectiveness, and many other issues of text composition became significant. Only a few writers would have sufficient skill to do these tasks competently, and these highly skilled writers began to specialize in particular domains, with increasingly few polymaths able to handle a broad spectrum of genres within different discursive domains. Today the genres of finance are far from those of poetry and both are far from the genres of sociology or medicine. Even our widely read, publicly distributed genres, like those of journalism or television scripts, are produced by a small subset of specialized writers. Although a few individuals gain some skill at multiple genres, it is near impossible to gain competence in more than a handful.

This brings us to our third level of challenge, as students move beyond the academy to participate in social roles in the workplace, community, politics, or other domains. Outside school, the purposes, tasks, the social roles and relations, genres, relevant knowledges and intertexts, the uses made of information, registers, and stances are widely varied and distinct from the world of schooling. In schooling, learning and display of learning are at the center of most transactions, and evaluation standards and procedures are largely explicit. 
The student's work is scaffolded by the setting; the world the text travels in is defined, local, and known in the classroom-with the addition of highly structured anonymous assessments. Beyond schooling, there is little guarantee that people will notice what you write, and little definition of the criteria by which people will evaluate your work, dismiss it, or pay attention. Writing in school usually has few consequences for the student beyond progression through the system (as long as the writing does not raise health and safety issues), while writing outside school has the potential of travelling far and having major consequences, whether for good or ill.

Even within the contained and relatively safe world of the classroom, students may need to learn to cope productively with anxieties raised by how their writing will be perceived and evaluated by their teachers, assessors, and peers. Teachers, however, can also work to create positive atmospheres for writing, increase trust, and diminish anxiety. As people begin to write in the larger world with substantial stakes and less supportive readers, the potential for anxiety is greater. Anxiety, when unmanaged, can interfere with the clarity of thought necessary for difficult writing, and can even steer a writer away from taking on a needed writing task. The writer needs to learn to see clearly through anxiety to gather confidence and courage to write what needs to be written. Part of the anxiety comes from the way people make judgments about ethnicity, class, education, creativity, and intelligence on the basis of one's writing. But also a large part of the anxiety is whether others will attend to, take seriously, and understand what it is one has written, and then further whether others will approve.

Writing, as well, puts the writer, so to speak, on the line with some permanence and consequence. Even when filling out a familiar form, the writer may be anxious whether one has filled in the correct dates and information on a non-refundable ticket, or has filed accurate information with a government agency. As a writer matures and understands how information flows and its consequences, he or she may become anxious about expressing private beliefs or unorthodox experiences in a document that might circulate. Even personal writing can be used as a witness against oneself, carrying social stigma or political danger. So management of anxiety and the wisdom to make wisely courageous choices also presents another level of challenge.

Finally, as the writer's resources, options, knowledge, and experience grow, the writer needs to become more explicitly aware of exactly what he or she wants to accomplish and how to go about it. It is one thing to fill out a few factual items in response to a questionnaire and quite another to put together extensive legal findings and facts into a coherent and effective legal brief structured by one's advocacy role for the client. It is even another, on the basis of an investigation, 
to come to conclusions about the policies that would be best for a community and then argue effectively for them. The writer must come to know his or her increasingly complex mind, and how to pull together all the external resources, make sense of them, and then from internal depths, externalize thoughts into a public document. Understanding and management of writing processes must continue to develop as the writer's repertoire and complexity of tasks expands, but in a way that maintains places for spontaneity, invention, and the force of complex unconscious and intuitions to direct the core of the message. If management of writing processes turns into mechanical algorithms, the text can lose immediacy, message, and interest. The process must be driven by the writer's fundamental communicative impulses to be saying something that he or she wants to say to a specific audience.

Learning to write is learning to navigate and act effectively within the complex social world we humans have historically created with literacy-or at least the small contemporary quarter of it relevant to the writer's life. After five millennia of writing-where literacy has become intertwined with almost every human activity from the medical monitoring of involuntary heartbeat to global agreements for the coordination of economies - the resources and tasks of writing are daunting. We must seek our understanding of writing in barely charted and swelling universes, where each new act creates new territory and expands the universe, where each writer must find new bearings and weave fresh nets to engage minds that transiently pass within reach of their inscribed words. Even the simplest act of writing is not predetermined and involves choices. We cannot then say there is any one answer about how to write. All we can do is to try to be wise about social, psychological, and historical processes, about our resources and responsibilities, about our opportunities and interests, to make our best choices in the protean and evanescent world of communication. The tasks are never ending and never the same. The results are rarely certain. Yet each successful act of writing increases our presence, our reach, our place in the world. And each act of writing makes the world a more habitable and inhabited place. 


\section{REFERENCES}

Addams, J. (1997). A Function of the Settlement House. In L. Menand (Ed.), Pragmatism: A reader (p. 273-286). New York: Vintage.

Adler, A. (1907). Studie über Minderwertigkeit von Organen. Vienna: Urban \& Schwarzenberg.

Albertini, J. (2008). Teaching of writing and diversity: Access, identity, and achievement. In C. Bazerman (Ed.), Handbook of research on writing: History, society, school, individual, and text (pp. 383-393). New York: Routledge.

Alexander of Villedieu. (1199). Doctrinale.

Althusser, L. (1971). Ideology and ideological state apparatuses. In Lenin and philosophy and other essays (pp. 121-76). New York: Monthly Review Press.

Anderson, J. (2008). The collection and organization of written knowledge. In C. Bazerman (Ed.), Handbook of research on writing: History, society, school, individual, text (pp. 177-190). New York: Routledge.

Aristotle (1991). On rhetoric: a theory of civic discourse (G. A. Kennedy, Trans.). New York: Oxford University Press.

Ashmore, M. (1993). The theatre of the blind: Starring a Promethean prankster, a phony phenomenon, a prism, a pocket, and a piece of wood. Social Studies of Science, 23, 67-106.

Atkinson, D. (1999). Scientific discourse in socio-historic context. Mahwah, NJ: Erlbaum.

Austin, J. (1962). How to do things with words. Oxford: Clarendon.

Bacon, F. (1605). The advancement of learning. London

Bakhtin, M. M. (1981) The dialogic imagination. Austin: University of Texas Press.

Bakhtin, M. (1984a). Problems of Doestoevsky's poetics. Minneapolis: University of Minnesota Press.

Bakhtin, M. (1984b). Rabelais and his world. Bloomington: Indiana University Press.

Bakhtin, M M. (1986) The problem of speech genres. In C. Emerson \& M. Holquist (Eds.), Vern W. McGee (trans.), Speech Genres and Other Late Essays (pp. 60-102). Austin: University of Texas Press.

Bakhtin, M. (1990). Art and answerability: Early philosophical essays. Austin: University of Texas Press.

Bakhtin, M. (1993). Toward a philosophy of the act. Austin: University of Texas Press.

Barthes, R. (1977). Image-music-text. London: Fontana. 
Bates, E., \& Goodman, J. (1997). On the inseparability of grammar and the lexicon: Evidence from acquisition, aphasia and real-time processing. Language and Cognitive Processes, 12(5/6), 507-586.

Bates, E., \& Goodman, J. (1999). On the emergence of grammar from the lexicon. In B. MacWhinney (Ed.), The emergence of language (pp. 29-79). Mahwah, NJ: Lawrence Erlbaum.

Baumann, R. (1986). Story, performance, and event: Contextual studies of oral narrative. Cambridge: Cambridge University Press.

Bazerman, C. (1984a). Modern evolution of the experimental report: Spectroscopic articles in Physical Review, 1893-1980. Social Studies of Science, 14, 163-96.

Bazerman, C. (1984b) The writing of scientific non-fiction: Contexts, choices and constraints. Pre/Text, 5(1), 39-74.

Bazerman, C. (1985). Physicists Reading Physics: Schema-Laden Purposes and Purpose-Laden Schema. Written Communication, 2(1), 3-23.

Bazerman, C. (1987a). Codifying the social scientific style: The APA Publication Manual as a behaviorist rhetoric. In J. Nelson, A. Megill, \& D. McCloskey (Eds.), The rhetoric of the human sciences (pp. 125-144). Madison: University of Wisconsin Press.

Bazerman, C. (1987b). Literate acts and the emergent social structure of science. Social Epistemology, 1(4), 295-310.

Bazerman, C. (1988). Shaping written knowledge: The genre and activity of the experimental article in science. Madison: University of Wisconsin Press.

Bazerman, C. (1991). How natural philosophers can cooperate: The rhetorical technology of coordinated research in Joseph Priestley's History and Present State of Electricity. In C. Bazerman \& J. Paradis (Eds.), Textual dynamics of the professions (pp. 13-44). Madison: University of Wisconsin Press.

Bazerman, C. (1993a). Intertextual self-fashioning: Gould and Lewontin's representations of the literature. In J. Selzer (Ed.), Understanding scientific prose (pp. 20-41). Madison: University of Wisconsin Press.

Bazerman, C. (1993b). Money talks: The rhetorical project of Adam Smith's Wealth of Nations. In W. Henderson et al. (Eds.), Economics and language (pp. 173-199). New York: Routledge.

Bazerman, C. (1994a). Constructing experience. Carbondale: Southern Illinois University Press.

Bazerman, C. (1994b). Systems of genre and the enactment of social intentions. In A. Freedman \& P. Medway (Eds.), Genre and the new rhetoric (pp. 79101). London: Taylor \& Francis.

Bazerman, C. (1994c). Whose moment? The kairotics of intersubjectivity. In Constructing experience. Carbondale: Southern Illinois University Press. 
Bazerman, C. (1997). Discursively structured activities. Mind, Culture and Activity, 4(4), 296-308.

Bazerman, C. (1999a). The languages of Edison's light. Cambridge, MA: MIT Press.

Bazerman, C. (1999b). Letters and the social grounding of differentiated genres. In D. Barton \& N. Hall (Eds.), Letter writing as a social practice (pp. 15-30). Amsterdam: Benjamins.

Bazerman, C. (2000a). A rhetoric for literate society: The tension between expanding practices and restricted theories. In M. Goggin (Ed.), Inventing a Discipline (pp. 5-28). Urbana: NCTE.

Bazerman, C. (2000b). Singular utterances: Realizing local activities through typified forms in typified circumstances. In A. Trosberg (Ed.), Analysing the discourses of professional genres (pp. 25-40). Amsterdam: Benjamins.

Bazerman, C. (2001a). Anxiety in action: Sullivan's interpersonal psychiatry as a supplement to Vygotskian psychology. Mind, Culture and Activity, 8(2), 174-186.

Bazerman, C. (2001b). Writing as a development in interpersonal relations. Journal for the Psychoanalysis of Culture and Society, 6(2), 298-302.

Bazerman, C. (2005). Practically human: The pragmatist project of the interdisciplinary journal Psychiatry. Linguistics and the Human Sciences, 1(1), 15-38.

Bazerman, C. (2006). The writing of social organization and the literate situating of cognition: Extending Goody's social implications of writing. In D. Olson \& M. Cole (Eds.), Technology, literacy and the evolution of society: Implications of the work of Jack Goody (pp. 215-240). Mahwah NJ: Erlbaum.

Bazerman, C. (Ed.). (2008). Handbook of research on writing: History, society, school, individual, and text. New York: Routledge.

Bazerman, C. (2009). Genre and cognitive development. In C. Bazerman, A. Bonini, \& D. Figueiredo (Eds.), Genre in a changing world (pp. 279-294). Fort Collins, Colorado: The WAC Clearinghouse and Parlor Press.

Bazerman, C. (2012). Writing with concepts: Communal, internalized, and externalized. Mind, Culture and Activity 19(3), 259-272.

Bazerman, C. \& de los Santos, R. (2005). Measuring incommensurability: Are toxicology and ecotoxicology blind to what the other sees? In R. Harris (Ed.), Rhetoric and incommensurability (pp. 424-463). West Lafayette, IN: Parlor Press.

Bazerman, C. \& Rogers P. (2008a). Writing and secular knowledge apart from modern European institutions. In C. Bazerman (Ed.), Handbook of research on writing: History, society, school, individual, and text (pp. 143-156). New York: Routledge. 
Bazerman, C. \& Rogers P. (2008b). Writing and secular knowledge within modern European institutions. In C. Bazerman (Ed.), Handbook of research on writing: History, society, school, individual, and text (pp. 157-176). New York: Routledge.

Beaufort, A. (2008). Writing in the professions. In C. Bazerman (Ed.). Handbook of research on writing: History, society, school, individual, text (pp. 217-232). New York: Routledge.

Berger, P., \& Luckmann, T. (1966). The social construction of reality. Garden City, NY: Doubleday.

Bergmann, J. (1993). Discreet indiscretions. New York: Aldine De Gruyter.

Bergmann, J., \& Luckmann, T. (1995). Reconstructive genres of everyday communication. In U. Quasthoff (Ed.), Aspects of oral communication. Berlin: Walter de Gruyter.

Blasi, A. (1998). George Herbert Mead's transformation of his intellectual context. In L. Tomasi (Ed.), The tradition of Chicago sociology. Aldershot: Ashgate.

Bloomfield, L. (1914). Introduction to the study of language. New York: Henry Holt.

Bourdieu, P. (1977). Outline of a theory of practice. Cambridge: Cambridge University. Press

Bourdieu, P. (1984). Distinction: A social critique of the judgment of taste. Cambridge, MA: Harvard University Press.

Bourdieu, P. (1990) The logic of practice. Stanford, CA: Stanford University Press.

Bourdieu, P. (1991). Language \& symbolic power. Cambridge, MA: Harvard University Press.

Bourdieu, P. (1993). The field of cultural production. New York: Columbia University Press.

Brooks, C. (1947). The well-wrought urn: Studies in the structure of poetry. Boston: Houghton Mifflin Harcourt.

Buber, M. (1937). I and Thou. New York: Charles Scribner's Sons.

Bulmer, M. (1986). The Chicago school of sociology. University Of Chicago Press. Burke, K. (1950). A Rhetoric of Motives. University of California Press.

Bybee, J. (2010). Language, usage and cognition. Cambridge: Cambridge University Press.

Calhoun, C. (2006). Pierre Bourdieu and social transformation: Lessons from Algeria. Development and Change 37(6), 1403-1415.

Chafe, W. (1994). Discourse, consciousness, and time: The flow and displacement of conscious experience in speaking and writing. Chicago: The University of Chicago Press.

Chomsky, N. (1965). Aspects of the theory of syntax. Cambridge: MIT Press. 
Clark, H. H. (1996). Using language. Cambridge: Cambridge University Press. Cole, M. (1996). Cultural psychology: A once and future discipline. Cambridge: Harvard University Press.

Collins, H. (1985). Changing order: Replication and induction in scientific practice. Beverly Hills: Sage.

Collins, J. (2011). Indexicalities of language contact in an era of globalization: Engaging John Gumperz' legacy. Text and Talk, 31(4), 407-428.

Conboy, M. (2008). Writing and journalism: Politics, social movements, and the public sphere. In C. Bazerman (ed.). Handbook of Research on Writing: History, Society, School, Individual, Text (pp. 205-220). New York: Routledge.

Coser. R. L. (1966). Role distance, sociological ambivalence, and transitional status systems. American Journal of Sociology, 72(2), 173-187.

Coser, R. L. (1975). The complexity of roles as a seedbed of individual autonomy. In L. Coser (Ed.), The idea of social structure. New York: Harcourt.

Coulmas, F. (1996). The Blackwell encyclopedia of writing systems. Oxford: Blackwell.

Cozzens, S. (1985). Comparing the sciences: Citation context analysis of papers from neuropharmacology and the sociology of science. Social Studies of Science, 15, 127-53.

Daniels, H. (Ed.) (1996). An introduction to Vygotsky. London: Routledge.

Daniels, H., Wertsch, J., \& Cole, M. (Eds.) (2007). The Cambridge companion to Vygotsky. Cambridge: Cambridge University Press.

Daniels, P. T., \& Bright, W. (1996). The World's writing systems. Oxford: Oxford University Press.

Darnell, R. (1989). Edward Sapir: linguist, anthropologist, humanist. Berkeley: University of California Press.

Deacon, T. (1997). The symbolic species: The co-evolution of language and the brain. New York: W.W. Norton \& Company.

Dear, P. (1985). Totius in verba: Rhetoric and authority in the early royal society. Isis, 76, 145-61.

Delamont, S., \& Atkinson, P. (2001). Doctoring uncertainty: Mastering craft knowledge. Social Studies of Science, 31(1), 87-107

De Bellis, N. (2009). Bibliometrics and citation analysis: From the science citation index to cybermetrics. Lanham, MD: Scarecrow Press.

De los Santos, R. (2007). Nation building as rhetoric and socio-cultural activity: Two institutional moments in post-revolutionary Mexico, 1928-1940 (Doctoral dissertation). Available from ProQuest Dissertations \& Theses database. (UMI No. 3283759)

De Man, P. (1983). Blindness and insight: Essays in the rhetoric of contemporary criticism. Minneapolis: University of Minnesota Press. 
Derrida, J. (1981). Writing and difference. London: Routledge \& Kegan Paul.

Devitt, A. (1991). Intertextuality in tax accounting: Generic, referential, and functional. In C. Bazerman \& J. Paradis (Eds.), Textual dynamics of the professions (pp. 336-380). Madison: University of Wisconsin Press.

Dewey, J. (1896). The reflex arc concept in psychology. Psychological Review, 3, 357-370.

Dewey, J. (1897). My pedagogic creed. School Journal, 54, 77-80

Dewey, J. (1910). How we think. Boston: D. C. Heath.

Dewey, J. (1922). Habits as social functions. Human nature and conduct: An introduction to social psychology (pp. 14-23). New York: Modern Library.

Dewey, J. (1947). Experience and education. New York: Macmillan.

Dickstein, M. (Ed.) (1998). The revival of pragmatism: New essays on social thought, law, and culture. Durham NC: Duke University Press.

Donald, M. (1991). Origins of the modern mind: Three stages in the evolution of culture and cognition. Cambridge, MA: Harvard.

Duranti, A., \& Goodwin, C. (Eds.) (1992). Rethinking context: Language as an interactive phenomenon. Cambridge: Cambridge University Press.

Elman, J., Bates, E., Johnson, M., Karmiloff-Smith, A., Parisi, D. \& Plunkett, K. (1996). Rethinking innateness: A connectionist perspective on development. Cambridge, MA: MIT Press/Bradford Books.

Empson, W. (1947). Seven types of ambiguity. NY: New Directions.

Engeström, Y. (1987). Learning by expanding: An activity-theoretical approach to developmental research. Helsinki: Orienta-Konsultit.

Engeström, Y. (1993). Developmental studies of work as a testbench of activity theory: The case of primary care medical practice. In S. Chaiklin \& J. Lave (Eds.), Understanding practice: Perspectives on activity and context (pp. 64-103). Cambridge: Cambridge University Press.

Engeström, Y., Brown, K., Christopher, L. C., \& Gregory, J. (1997). Coordination, cooperation, and communication in the courts: Expansive transitions in legal work. In M. Cole, Y. Engestrom, \& O. Vasquez (Eds.) Mind, culture, and activity: Seminal papers from the Laboratory of Comparative Human Cognition. Cambridge: Cambridge University Press.

Engeström, Y., \& Escalante, V. (1995). Mundane tool or object of affection? The rise and fall of the Postal Buddy. In B. Nardi (Ed.), Context and consciousness: Activity theory and human-computer interaction (pp. 335-373). Cambridge, MA: MIT Press.

Faris, R. (1979). Chicago sociology, 1920-1932. Chicago: University of Chicago Press. 
Feffer, A. (1993). The Chicago pragmatists and American progressivism. Ithaca, NY: Cornell University Press.

Fish, S. (1980). Is there a text in this class? The authority of interpretive communities. Cambridge, MA: Harvard University Press.

Fleck, L. (1979). Genesis and development of a scientific fact. Chicago: University of Chicago Press.

Foucault, M. (1970). The order of things: An archaeology of the human sciences. New York: Vintage Books.

Fromm, E. (1961). Marx's concept of man. New York: Ungar.

Gadamer, H-G. (1975). Hermeneutics and social science. Philosophy Social Criticism/Cultural Hermeneutics, 2, 307-316.

Garfinkel, H. (1967). Studies in ethnomethodology. Englewood Cliffs, NJ: Prentice-Hall.

Geertz, C. (1980). Negara: The theatre state in nineteenth-century Bali. Princeton: Princeton University Press.

Gelb, I. J. (1952). A study of writing. Chicago: University of Chicago Press.

Genette, G. (1992). The architext. Berkeley: University of California Press.

Genette, G. (1997a). Palimpsests. Lincoln: University of Nebraska Press.

Genette, G. (1997b). Paratexts. Cambridge: Cambridge University Press.

Giddens, A. (1984). The constitution of society. Berkeley: University of California Press.

Giddens, A. (1987). Social theory and modern sociology. Cambridge: Polity Press. Gilbert, N., \& Mulkay, M. (1984). Opening Pandora's box: A sociological analysis of scientists' discourse. Cambridge: Cambridge University Press.

Goffman, E. (1959). The presentation of self in everyday life. New York: Anchor Books.

Goffman, E. (1963). Stigma. Englewood Cliffs, NJ: Prentice Hall.

Goffman, E. (1971). Relations in public. New York: Basic Books.

Goffman, E. (1974). Frame Analysis. New York: Harper Colophon.

Goffman, E. (1981). Forms of talk. Philadelphia: University of Pennsylvania Press.

Goffman, E. (1983). The interaction order. American Sociological Review, 48. $1-17$.

Gogtay, N. et al. (2004). Dynamic mapping of human cortical development during childhood through early adulthood. PNAS, 101(2), 8174-8179.

Goodwin, C. (1984). Notes on story structure and the organization of participation. In J. M. Atkinson \& J. Heritage (eds.), Structures of Social Action (pp. 225-246). Cambridge: Cambridge University Press.

Goodwin, C. (1994). Professional Vision. American Anthropologist, 96(3), 606-33. 
Goody, J. (1977). The domestication of the savage mind. Cambridge: Cambridge University Press.

Goody, J. (1986). The logic of writing and the organization of society. Cambridge: Cambridge University Press.

Graham, S. (2006). Strategy instruction and the teaching of writing: A metaanalysis. In C. MacArthur, S. Graham, \& J. Fitzgerald (Eds.), Handbook of writing research (pp. 187-207). New York: Guilford.

Gross, A. G., Harmon, J. E., \& Reidy, M. S. (2002). Communicating science: The scientific article from the 17th century to the present. Oxford: Oxford University Press.

Gross, A., \& Keith, W. (Eds.) (1996). Rhetorical hermeneutics: Invention and interpretation in the age of science. Albany: SUNY Press.

Günthner, S., \& . (1995). Culturally patterned speaking practices-The analysis of communicative genres. Pragmatics, 5(1), 1-32.

Gumperz, J. (1982). Discourse strategies. Cambridge: Cambridge University Press.

Gumperz, J. (1992). Contextualization and understanding. In A. Duranti \& C. Goodwin (Eds.), Rethinking context (pp. 229-252). Cambridge: Cambridge University Press.

Halliday, M. A. K., \& Matthiessen, C. M. I. M. (2004). An introduction to functional grammar (3d ed.). London: Arnold.

Halliday, M. A. K., \& Hasan, R. (1976). Cohesion in English. London: Longman.

Halliday, M. A. K., \& Martin, J. (1993). Writing science. London: Taylor \& Francis.

Hanks, W. (1990). Referential practice. Chicago: University of Chicago Press.

Hanks, W. (1996). Language and communicative practices. Boulder, CO: Westview.

Harris, R. (2000). Rethinking writing. London: The Athlone Press.

Harris, R. (1981). The language myth. New York: St. Martin's Press.

Harris, R. (1987). Reading Saussure: A critical commentary on the Cours de linguistique générale. London: Duckworth.

Harris R. A. (Ed.) (2005). Rhetoric and incommensurability. West Lafayette IN: Parlor Press.

Havelock, E. (1971). Prologue to Greek Literacy. Cincinnati: University of Cincinnati Press.

Havelock, E. (1981). The literate revolution in Greece and its cultural consequences. Princeton, NJ: Princeton University Press.

Heath, S. B. (1983). Ways with words: Language, life and work in communities and classrooms. Cambridge University Press. 
Heidegger, M. (1962). Being and time. New York: Harper \& Row.

Hemingway, E. (1958). The art of fiction No. 21. Interviewed by George Plimpton. Paris Review 18.

Heritage, J. (1984). Garfinkel and ethnomethodology. New York: Polity Press.

Hillocks, G. (1986). Research on written composition: New directions for teaching. Urbana, IL: National Conference on Research in English.

Hogan, P. (2008). Writing as art and entertainment. In C. Bazerman (Ed.) Handbook of research on writing: History, society, school, individual, text (pp. 191-204). New York: Routledge.

Hopper, P., \& Traugott, E. (1993). Grammaticalization. Cambridge: Cambridge University Press.

Howe, M. D. W. (1957). Justice Oliver Wendell Holmes: The proving years, 18701882. Belknap Press.

Husserl, E. (1964). The Idea of Phenomenology. Hague: Martinus Nijhoff.

Hutchins, E. (1995). Cognition in the wild. Cambridge, MA: MIT Press.

Inglese, T. (2010). Can archived TV interviews with social science scholars enhance the quality of students' academic writing? In C. Bazerman et al. (Eds.), Traditions of writing research (pp. 309-324). New York. Routledge.

Iser, W. (1980). The act of reading: A theory of aesthetic response. Baltimore: Johns Hopkins University Press.

James, W. (1890). Principles of psychology (2 vols.). New York: Holt.

James, W. (1912). Essays in Radical Empiricism. New York: Longman Green and Co.

Joas, H. (1985). G. H. Mead: A contemporary re-examination of his thought. Cambridge, MA: MIT Press.

Joas, H. (1993). Pragmatism and social theory. Chicago: University of Chicago Press.

Johnson, C. M., \& Karin-D’Arcy, M. R. (2006). Social attention in nonhuman primates: A behavioral review. Aquatic Mammals, 32(4), 423-442.

Johnson, S. (1755). A Dictionary of the English Language. London.

Kasanin, J. (Ed.). (1944). Language and thought in schizophrenia. University of California Press.

Kilpatrick, W. H. (1951). Philosophy of education. New York: Macmillan Co. Kozulin, A. (1990). Vygotsky's psychology: A biography of ideas. Cambridge, MA: Harvard University Press.

Kristeva, J. (1980). Desire in language: A semiotic approach to literature and art. New York: Columbia University Press.

Kuhn, T. S. (1962). The structure of scientific revolutions. Chicago: University of Chicago Press.

Latour, B. (1987). Science in action. Cambridge, MA: Harvard University Press. 
Latour, B., \& Woolgar, S. (1979). Laboratory life. Beverly Hills: Sage.

Lave, J., \& Wenger, E. (1991). Situated learning: Legitimate peripheral participation. Cambridge: Cambridge University Press.

Leont'ev, A. N. (1978). Activity, consciousness, and personality. Englewood Cliffs, NJ: Prentice-Hall.

Leont'ev, A. N. (1981). Problems of the development of the mind. Moscow: Progress.

Lerner, G. H. (1993). Collectivities in action: Establishing the relevance of conjoined participation in conversation. Text, 13(2), 213-245.

List of Language Regulators. (2012). In Wikipedia. Retrieved from http:// en.wikipedia.org/wiki/List_of_language_regulators

Lowth, R. (1762). A Short Introduction to English Grammar. London.

Luckmann, T. (1995). On the communicative adjustment of perspectives, dialogue and communicative genres. In E. Goody (Ed.), Social Intelligence and Interaction: Expressions and Implications of the Social Bias in Human Intelligence (pp. 175-186). Cambridge: Cambridge University Press.

Luhmann, N. (1983). The differentiation of society. New York: Columbia University Press.

Luhmann, N. (1995). Social systems. Stanford, CA: Stanford University Press.

Luria, A. R. (1961). The role of speech in the regulation of normal and abnormal behavior. New York: Pergamon.

Luria, A. R. (1968). The mind of a mnemonist. Cambridge, MA: Harvard University Press.

Luria, A. R. (1969). Speech development and the formation of mental processes. In M. Cole \& I. Maltzman (Eds.), A handbook of contemporary soviet psychology (pp. 121-162). New York: Basic Books.

Luria, A. R. (1970). The functional organization of the brain. Scientific American, 222 (3), 66-78.

Luria, A. R. (1972). The man with a shattered world. Cambridge, MA: Harvard University Press.

Luria, A. R. (1976). Cognitive development: Its cultural and social foundations. Cambridge, MA: Harvard University Press.

Luria, A. R. (1978). The selected writings of A.R. Luria. White Plains: M. E. Sharpe.

Luria, A. R. (1979). The making of mind: A personal account of Soviet psychology. Cambridge, MA: Harvard University Press.

Luria, A. R., \& Yudovich, R. (1959). Speech and the development of mental processes in the child. London: Staples Press.

McCarthy, L. P. (1991). A psychiatrist using DSM-III: The influence of a charter document in psychiatry. In C. Bazerman \& J. Paradis (Eds.), Textual 
dynamics of the professions (pp. 358-378). Madison: University of Wisconsin Press.

McCarthy, L. P., \& Gerring, J. P. (1994). Revising psychiatry's charter document, DSM-IV. Written Communication, 11(2), 147-192.

Marx, K. (1909). Capital: Critique of political economy. Chicago: Charles H. Kerr.

Marx, K. (1963). The eighteenth brumaire Of Louis Bonaparte. New York: International Publishers.

Marx, K., \& Engels, F. (1970). The German ideology (3d rev. ed.). Moscow: Progress Publishers.

Matthews, F. (1977). Quest for American sociology: Robert E. Park and the Chicago school. Montreal: McGill-Queen's University Press.

Mauss, M. (1922). The Gift: forms and functions of exchange in archaic societies. London: Routledge.

McMurry, C. A. (1920) Teaching by projects: A basis for purposeful study. New York: Macmillan.

Mead, G. H. (1913). The social self. Journal of Philosophy, Psychology and Scientific Methods, 10, 374-380.

Mead, G. H. (1929). A pragmatic theory of truth. Studies in the Nature of Truth. University of California Publications in Philosophy 11 (pp. 6588). Berkeley: University of California Press.

Mead, G. H. (1934). Mind, self, and society. Chicago: University of Chicago Press. Mead, G. H. (1936). The problem of society: How we become selves. In M. H. Moore (Ed.), Movements of thought in the nineteenth century (pp. 360-385). Chicago: University of Chicago Press.

Medawar, P. B. (1964). Is the scientific paper fraudulent? Saturday Review, 1, 42-43.

Medvedev, P. N. (1978). The formal method in literary scholarship. Baltimore: Johns Hopkins University Press.

Menand, L. (1997). Pragmatism: A reader. New York: Vintage.

Menand, L. (2001). The metaphysical club: A story of ideas in America. New York: Farrar, Straus, and Giroux .

Merton, R. K. (1936). The unanticipated consequences of purposive social action. American Sociological Review, 1, 894-904.

Merton, R. K. (1938a). Science, technology and society in seventeenth century England. OSIRIS: Studies on the History and Philosophy of Science and on the History of Learning and Culture, IV(2), 360-632. Bruges, Belgium: St. CatherinePress.

Merton, R. K. (1938b). Social structure and anomie. American Sociological Review, 3, 672-682. 
Merton, R. K. (1940). Bureaucratic structure and personality. Social Forces, 18, 560-568.

Merton, R. K. (1945). Role of the intellectual in public bureaucracy. Social Forces, 23, 405-415.

Merton, R. K. (1948). The self-fulfilling prophecy. Antioch Review, 8, 193-210.

Merton, R. K. (1949). Social Structure and Anomie: Revisions and Extensions. In R. N. Anshen (Ed.), The family: Its functions and destiny (pp. 226-257). New York: Harper \& Brothers.

Merton, R. K. (1950a). Contributions to the theory of reference group behavior (with A. Rossi). In R. K. Merton \& P. F. Lazarsfeld, (Eds.) Continuities in social research (pp. 40-105). New York: The Free Press.

Merton, R. K. (1950b). Patterns of influence: A study of interpersonal influence and communications behavior in a local community. In P. F. Lazarsfeld \& F. Stanton (Eds.), Communications Research, 1948-49 (pp. 180-219). New York: Harper \& Brothers.

Merton, R. K. (1957a). Priorities in scientific discovery: A chapter in the sociology of science. American Sociological Review, 22(6), 635-659.

Merton, R. K. (1957b). The role-set: Problems in sociological theory. British Journal of Sociology, 8, 106-120.

Merton, R. K. (1959). Social conformity, deviation and opportunity structures. American Sociological Review, 24(2), 177-189.

Merton, R. K. (1961). Singletons and multiples in scientific discovery. Proceedings of the American Philosophical Society, 105(5), 470-486.

Merton, R. K. (1963). The ambivalence of scientists. Bulletin of the Johns Hopkins Hospital, 112, 77-97.

Merton, R. K. (1965). On the shoulders of giants: A Shandean postscript. New York: The Free Press.

Merton, R. K. (1968a). The Matthew effect in science: the reward and communication systems of science are considered (with H. A. Zuckerman). Science, 199, (3810), 55-63.

Merton, R. K. (1968b). Social theory and social structure, enlarged edition. New York: Free Press.

Merton, R. K. (1971). Patterns of evaluation in science: Institutionalization, structure and functions of the referee system (with $\mathrm{H}$. A. Zuckerman). Minerva, 9(1), 66-100.

Merton, R. K. (1973). Sociology of science. Chicago: University of Chicago Press.

Merton, R. K. (1976). Sociological ambivalence. New York: The Free Press.

Merton, R. K. (1989). Unanticipated consequences and kindred sociological ideas: A personal gloss. In C. Mongardini \& S. Tabboni (Eds.), L'Opera 
di Robert K. Merton e la sociologia contemporanea (pp. 307-329). Genova: ECIG.

Merton, R. K. (1995). The Thomas theorem and the Matthew effect. Social Forces, $74(2), 379-424$.

Merton, R. K., Coleman, J. S., \& Rossi, P. (Eds.) (1979). Qualitative and quantitative social research: Papers in honor of Paul F. Lazarsfeld. New York: The Free Press.

Merton, R. K., Reader, G., \& Kendall, P. (Eds.) (1957). The student physician. Cambridge MA: Harvard University Press.

Miller, C. (1984). Genre as social action. Quarterly Journal of Speech, 70, 151-67. Miller, C. (1992). Kairos in the rhetoric of science. In S. Witte et al. (Eds.), $A$ rhetoric of doing (pp. 310-27). Carbondale: Southern Illinois University Press.

Moss, J. D. (1993). Novelties in the heavens. University of Chicago Press.

Murphy, J. J. (1971). Three medieval rhetorical arts. Berkeley: University of California Press.

Myers, G. (1991). Stories and styles in two molecular biology articles. In C. Bazerman \& J. Paradis (Eds.), Textual dynamics of the professions (pp. 45-75). Madison: University of Wisconsin Press.

Natanson, M. (1956). The social dynamics of G. H. Mead. Washington D.C.: Public Affairs Press.

Nietzsche, F. (2008). The birth of tragedy. Oxford: Oxford University Press.

Ochs, E., Schegloff, E., \& Thompson, S. (Eds.) (1996). Interaction and grammar. Cambridge: Cambridge University Press.

Ong, W. (1958). Ramus, method, and the decay of dialogue: From the art of discourse to the art of reason. Cambridge, MA: Harvard University Press.

Ong, W. (1982). Orality and literacy: The technologizing of the word. New York: Routledge.

Olson, D. (2008). History of schools and writing. In C. Bazerman (Ed.) Handbook of research on writing: History, society, school, individual, text (pp. 283-292). New York: Routledge.

Paus, T. (1999). Structural Maturation of Neural Pathways in Children and Adolescents: In Vivo Study. Science, 283(5409), 1908-1911.

Peirce, C. (1958). The collected papers. Volume 8. Cambridge, MA: Harvard University Press.

Perelman, L. (1991). The medieval art of letter writing: Rhetoric as institutional expression. In C. Bazerman \& J. Paradis (Eds.), Textual Dynamics of the Professions (pp. 97-119). Madison: University of Wisconsin Press.

Petanjek, Z. et al. (2011). Extraordinary neoteny of synaptic spines in the human prefrontal cortex. PNAS, 108(32), 13281-13286 
Pfuetze, P. (1954). The social self. New York: Bookman.

Plimpton, G. (1958). Ernest Hemingway, The art of fiction No. 21. The Paris Review, 18. Retrieved from http://www.theparisreview.org/interviews/4825/ the-art-of-fiction-no-21-ernest-hemingway

Pohlman, H. L. (1984). Justice Oliver Wendell Holmes \& utilitarian jurisprudence. Cambridge, MA: Harvard University Press.

Prendergast, C. (1986). Alfred Schutz and the Austrian school of economics. American Journal of Sociology, 92, 1-26.

Prior, P., \& Lunsford, K. (2008). History of reflection, theory, and research on writing. In C. Bazerman (ed.) Handbook of research on writing: History, society, school, individual, text (pp. 81-96). New York: Routledge.

Radcliffe-Brown, A. R. (1922). The Andaman islanders. Cambridge: Cambridge University Press.

Radcliffe-Brown, A. R. (1931). Social organization of Australian tribes. Melbourne, Macmillan \& Co.

Richards, I. A. (1924). The principles of literary criticism. London: Kegan Paul, Trench, Trubner.

Richards, I. A. (1929). Practical criticism. London: Kegan Paul, Trench, Trubner. Riffaterre, M. (1984). Intertextual representation. Critical Inquiry, 11(1), 141-162.

Rogers, C. (1961). On becoming a person: A therapist's view of psychotherapy. London: Constable.

Rorty, R. (1979). Philosophy and the mirror of nature. Princeton, NJ: Princeton University Press.

Rosch, E. (1977). Human categorization. In Warren, N. (Ed.), Advances in cross-cultural psychology 1 (pp. 1-72). New York: Academic Press.

Rosenthal, S. B., \& Bourgeois, P. L. (1991). Mead and Merleau-Ponty: Toward a common vision. Albany: SUNY.

Rowe, D. (2009). Early written communication. In R. Beard, D. Myhill, J. Riley, \& M. Nystrand. The Sage handbook of writing development (pp. 213231). Los Angeles: Sage.

Ruestow, E. G. (1996). The microscope in the Dutch Republic: the shaping of discovery. Cambridge: Cambridge University Press.

Russell, D. (1991). Writing in the academic disciplines, 1870-1990: A curricular history. Carbondale: Southern Illinois University Press.

Russell, D. (1995). Activity theory and its implications for writing instruction. In J. Petraglia (Ed.), Reconceiving writing, rethinking writing insruction (pp. 51-77). Hillsdale, NJ: Erlbaum.

Russell, D. (1997a). Rethinking genre in school and society: An activity theory analysis. Written Communication, 14(4), 504-554. 
Russell, D. (1997b). Writing and genre in higher education and workplaces. Mind, Culture, and Activity, 4(4), 224-237.

Russell, D. (2010). Writing in Multiple Contexts: Vygotskian CHAT Meets the Phenomenology of Genre. In C. Bazerman et al. (Eds.), Traditions of writing research (pp. 353-364). New York: Routledge.

Sacks, H. (1995). Lectures on conversation (2 vols.). Oxford: Blackwell

Sacks, O. (1985). The man who mistook his wife for a hat. London: Duckworth. Sacks, O. (1989). Seeing voices. New York: Vintage.

Sacks, O. (1995). An anthropologist on Mars, New York: Alfred A. Knopf. Sacks, O. (1996). The island of the colorblind. New York: Alfred A. Knopf.

Sapir, E. (1912). Language and environment. American Anthropologist, 14, 226-242.

Sapir, E. (1917). A Freudian half-holiday, review of Sigmund Freud, Delusion and Dream. The Dial, 63, 635-637.

Sapir, E. (1923). The two kinds of human beings, review of C. G. Jung, Psychological Types or the Psychology of Individuation. The Freeman, 8, 211-212.

Sapir, E. (1927a). Speech as a personality trait. American Journal of Sociology, 32, 892-905.

Sapir, E. (1927b). The unconscious patterning of behavior in society. In E. S. Dummer, (Ed.), The unconscious: A symposium (pp. 114-142). New York.

Sapir, E. (1934a). The emergence of the concept of personality in a study of cultures. Journal of Social Psychology, 5, 408-415.

Sapir, E. (1934b). Personality. In E. R.A. Seligman (Ed.), Encyclopaedia of the Social Sciences, Vol. 12 (pp. 85-87). New York: Macmillan.

Sapir, E. (1935). Communication. In E. R.A. Seligman \& A. S. Johnson (Eds.), Encyclopaedia of the Social Sciences, Vol. 4 (pp. 78-81). New York: Macmillan. Sapir, E. (1938). Why cultural anthropology needs the psychiatrist. Psychiatry, 1,7-12.

Sapir, E. (1949). The status of linguistics as a science. In D. G. Mandelbaum (Ed.). Selected writings of Edward Sapir in language, culture, and personality. Berkeley: University of California Press.

Saussure, F. de. (1983). Course in general linguistics. LaSalle, IL: Open Court.

Scharer, P. L., \& Zutell, J. (2003) The development of spelling. In N. Hall, J. Larson, \& J. Marsh (Eds.), Handbook of early childhood literacy (pp. 271286). Thousand Oaks, CA: Sage.

Schegloff, E. A (1987). Between micro and macro: Contexts and other connections. In J. Alexander, B. Giesen, R. Munch \& N. Smelser (Eds.), The micro-macro link (pp. 207-234). Berkeley and Los Angeles: University of California Press. 
Schegloff, E. A (1996). Turn Organization: One Intersection of Grammar and Interaction. In E. Ochs, E. A. Schegloff, \& S. Thompson (Eds.), Interaction and grammar (pp. 52-133). Cambridge: Cambridge University Press.

Schmandt-Besserat, D. (1996). How writing came about. Austin: University of Texas Press.

Schryer, C. (1994). The lab vs. the clinic: Sites of competing genres. In A. Freedman \& P. Medway (Eds.), Genre and the new rhetoric (pp. 105-124). London: Taylor and Francis.

Schryer, C. (2002). Strategies for Stability and Change. In R. Coe \& T. Teslenko (Eds.), The rhetoric and ideology of genre (pp. 73-102). New York: Hampton Press. Schryer, C. et al. (2002). Structure and agency in medical case presentations. In Bazerman, C. \& Russell, D. (Eds.) Writing selves/writing societies: Research from activity perspectives. Fort Collins, Colorado: The WAC Clearinghouse.

Schutz, A. (1951). Making music together: A study in social relationship. Social Research, 18 (1), 76-97.

Schutz, A. (1967a). The phenomenology of the social world. Evanston, IL: Northwestern University Press.

Schutz, A. (1967b). The problem of social reality. The Hague: Martinus Nijhoff. Schutz, A., \& Luckmann, T. (1973). The structures of the life-world. Evanston, IL: Northwestern University Press.

Scribner, S., \& Cole, M. (1981). The psychology of literacy. Cambridge, MA: Harvard University Press.

Searle, J. R. (1969). Speech Acts. Cambridge: Cambridge University Press. Searle, J. R. (1983). Intentionality: An essay in the philosophy of mind. Cambridge: Cambridge University Press.

Searle, J. R. (1992). The Rediscovery of the Mind. Cambridge, MA: Bradford. Searle, J. R., \& Vanderveken, D. (1985). Foundations of illocutionary logic. Cambridge: Cambridge University Press.

Selting, M., \& Couper-Kuhlen, E. (Eds.) (2001). Studies in interactional linguistics. Amsterdam: Benjamins.

Shklar, J. N. (2004). Squaring the hermeneutic circle. Social Research, 71(3), 657-658.

Slobin, D. I. (1987). Thinking for speaking. Proceedings of the Thirteenth Annual Meeting of the Berkeley Linguistics Society, 435-445. Retrieved from http:// elanguage.net/journals/bls/article/view/2508/2475

Small, H. (1978). Cited documents as concept symbols. Social Studies of Science, $8,327-40$.

Smart, G. (1993). Genre as community invention: A central bank's response to its executives' expectations as readers. In R. Spilka (Ed.), Writing in the 
workplace: New research perspectives (pp. 124-140). Carbondale: Southern Illinois University Press.

Smart, G. (2003). A central bank's "communications strategy": The interplay of activity, discourse genres, and technology in a time of organizational change. In C. Bazerman and D. Russell (Eds.), Writing selves/writing societies: Research from activity perspectives (pp. 9-61). Fort Collins, Colorado: The WAC Clearinghouse.

Smart, G. (2006). Writing the economy: Activity, genre, and technology in the world of banking. London: Equinox.

Smart, G. (2008). Writing and the social formation of economy. In C. Bazerman (Ed.) Handbook of research on writing: History, society, school, individual, text (pp. 103-112). New York: Routledge.

Smith, A. (1976). An inquiry into the nature and causes of the wealth of nations (R. H. Campbell \& A. S. Skinner, Eds.). Oxford: Clarendon Press.

Smith, A. (1978). Lectures on jurisprudence (R. L. Meek, D. D. Raphael, \& F. G. Stein, Eds.). Oxford: Clarendon Press.

Smith, A. (1980). Essays on philosophical subjects (W. P. D. Wightman, Ed.). Oxford: Clarendon Press.

Smith, A. (1983). Lectures on rhetoric and belles lettres (J. C. Bryce, Ed.). Oxford: Clarendon Press.

Smith A. (1986). The theory of moral sentiments (D. D. Raphael \& A. L. Macfie, Eds.). Oxford: Clarendon Press.

Smith, D. E. (1990). Texts, facts, and femininity: Exploring the relations of ruling. London: Routledge

Smith, D. E. (2002). Texts and the ontology of organizations and institutions. Studies in Cultures, Organizations and Societies, 7(2), 159-198.

Smith, D. E., \& Schryer, C. (2008) On documentary society. In C. Bazerman (Ed.) Handbook of research on writing: History, society, school, individual, text (pp. 113-128). New York: Routledge.

Smith, T. V. (1931). The social philosophy of George Herbert Mead. American Journal of Sociology, 37(3), 368-85.

Sokal, A., \& Bricmont, J. (1999). Fashionable nonsense: Postmodern intellectuals' abuse of science. NY: Picador.

Stinchcombe, A. L. (1975). Merton's theory of social structure. In L. Coser (Ed.), The idea of social structure (pp. 11-34). New York: Harcourt Brace Jovanovich.

Street, B. V. 1985. Literacy in theory and practice. Cambridge: Cambridge University Press.

Sullivan, H. S. (1953). The interpersonal theory of psychiatry. New York: Norton. 
Swales, J. M. (1990). Genre analysis: English in academic and research settings. Cambridge: Cambridge University Press.

Swales, J. M. (2004). Research genres: Explorations and applications. Cambridge: Cambridge University Press.

Tanner, L. (1997). Dewey's laboratory school: Lessons for today. New York: Teachers' College Press.

Taubes, G. (1993). Bad science: The short life and weird times of cold fusion. New York: Random House.

Thomas, W.I. (1923). The unadjusted girl. Boston: Little, Brown \& Co.

Thomason, B. C. (1982). Making sense of reification: Alfred Schutz and constructionist theory. London: Macmillan.

Tiersma, P. (1999). Legal Language. Chicago: University of Chicago Press.

Tiersma, P. (2008). Writing, text, and the law. In C. Bazerman (Ed.) Handbook of research on writing: History, society, school, individual, text (pp. 125-138). New York: Routledge.

Tiersma, P. (2010). Parchment, paper, pixels: Law and the technologies of communication. Chicago: University of Chicago Press.

Todorov, T. (1990). Genres in discourse. Cambridge: Cambridge University Press.

Tomasello, M. (2006). Why don't apes point? In N. J. Enfield \& S. C. Levinson (Eds.), Roots of human sociality: Culture, cognition and interaction (pp. 506524). Oxford \& New York: Berg.

Tomasi, L. (Ed.) (1998). The tradition of Chicago sociology. Aldershot: Ashgate.

Van der Veer, R. (2007). Lev Vygotsky. London: Continuum.

Van der Veer, R., \& Valsiner, J. (1991). Understanding Vygotsky. A quest for synthesis. Oxford: Basil Blackwell.

Van der Veer, R., \& Valsiner, J. (Eds.) (1994). The Vygotsky Reader. Oxford: Blackwell.

Veresov, N. N. (1999). Undiscovered Vygotsky: Etudes on the pre-history of culturalhistorical psychology. New York: Peter Lang.

Vocate, D. R. (1987). The theory of A.R. Luria: Functions of spoken language in the development of higher mental processes. Hillsdale, NJ: Erlbaum.

Volosinov, V. N. (1973). Marxism and the philosophy of language. Cambridge MA: Harvard University Press.

Volosinov. V. N. (1987). Freudianism. Bloomington: Indiana University Press. Vygotsky, L. S. (1925). The methods of reflexological and psychological investigation. Metodika refleksologicheskogo i psikhologicheskogo issledovanija. In K. N. Kornilov (Ed.), Problemy sovremennoj psikhologii (pp. 26-46). Leningrad: Gosudarstevennoe Izdarel'stvo.

Vygotsky, L. S. (1939). Thought and speech. Psychiatry, 2(1), 29-52. 
Vygotsky, L. S. (1967). Play and its role in the mental development of the child. Soviet Psychology, 5(3), 6-18.

Vygotsky, L. S. (1971). The psychology of art. Cambridge MA: MIT Press.

Vygotsky, L. S. (1978). Mind in society: The development of higher psychological processes (M. Cole, V. John-Steiner, S. Scribner, \& E. Souberman, Eds.). Cambridge, MA: Harvard University Press.

Vygotsky, L. S. (1986). Thought and language (Alex Kozulin, Trans.). Cambridge: MIT Press.

Vygotsky, L. S. (1993). Fundamentals of defectology (J. Knox \& C. Stevens, Trans.). New York: Plenum Press.

Vygotsky, L. S. (1994) Adolescent pedagogy. In R. van der Veer \& J. Valsiner (Eds.), Vygotsky reader. Blackwell 1994;

Vygotsky, L. S. (1999). Consciousness as a problem in the psychology of behavior. In N. Veresov (Ed.), Undiscovered Vygotsky: Etudes on the pre-history of cultural-historical psychology (pp. 251-281). Bern: Peter Lang.

Vygotsky, L. S., \& Luria, A. R. (1993). Studies on the history of behavior: Ape, primitive, and child. Hillsdale, NY: Lawrence Erlbaum.

Wenger, E. (1998). Communities of practice: Learning, meaning, and identity. Cambridge: Cambridge University Press.

Wertsch, J. (1985). Vygotsky and the social formation of mind. Cambridge MA: Harvard University Press.

Wertsch, J. (1998). Mind as action. New York: Oxford University Press.

Wilkins, J. (1668). An Essay towards a real character and a philosophical language. London.

Wimsatt, W. K, \& Beardsley, M. (1946). The intentional fallacy, Sewanee Review, 54, 468-488.

Wimsatt, W. K, \& Beardsley, M. (1949). The affective fallacy. Sewanee Review, 57, 31-55.

Wittgenstein, L. (1922). Tractatus logico-philosophicus (C. K. Ogden, Trans.), London: Routledge \& Kegan Paul.

Wittgenstein, L. (1958). Philosophical investigations (G. E. M. Anscombe, Trans.). Oxford: Basil Blackwell.

Wynn, J. (2012). Evolution by the numbers: The origins of mathematical argument in biology. ParlorPress. 


\section{References}





\section{A THEORY OF LITERATE ACTION}

"A Theory of Literate Action makes a significant contribution to the field and enriches and deepens our perspectives on writing by drawing together such varied and wide-ranging approaches from social theory and the social sciences-from psychology, to phenomenology, to pragmatics-and demonstrating their relevance to writing studies. While much has been made of the 'social turn' in the field of Rhetoric and Composition, the impact of social theory and social sciences on rhetorical theory and literacy studies has not been as fully explored-nor have these approaches been gathered together in one comprehensive text, to my knowledge."

"I have followed Chuck Bazerman's thinking closely over the years, but seeing it all together allowed me to see what I had not seen in it: how cognitive psychology (even neurobiology) intersects with social psychology and then sociology; how attentional processes and motive/emotion relate to genre; the historical insights; all up and down, macro micro meso. This work leads in so many productive directions. I've taken pages of notes."

\section{- David R. Russell}

Charles Bazerman, Professor of Education at the University of California, Santa Barbara, is the author of numerous research articles and books on the social role of writing, academic genres, and textual analysis, as well as textbooks on the teaching of writing.

Perspectives on Writing

Series Editor, Susan H. McLeod

The WAC Clearinghouse Fort Collins, CO 80523-1052

wac.colostate.edu

\section{Parlor Press}

Anderson, SC 29621

www.parlorpress.com 